\section{Breast Pathology}

125

Atypical Squamous Metaplasia: A Common Manifestation of Acute Radiation Injury in the Female Breast and Possible Diagnostic Pitfall $S$ Abbott, J Horton, J Geradts. Duke University Medical Center, Durham, NC.

Background: Traditional breast conservation therapy consists of partial mastectomy followed by radiotherapy. Thus, previous studies of radiation-induced changes in the breast have focused on residual extratumoral breast tissue that remains after excision, often long after radiotherapy. A current phase 1 trial of single-dose pre-operative radiotherapy followed by partial mastectomy has yielded a novel specimen type - acutely irradiated primary breast tumor and surrounding benign breast tissue. Review of such cases revealed a high rate of atypical squamous metaplasia (ASM).

Design: The ongoing trial has recruited 32 women with clinical stage Tis/T1N0M0 breast cancer, predominantly invasive ductal carcinoma and DCIS. Three cohorts of 8,8 , and 16 women received single radiotherapy doses of $15 \mathrm{~Gy}, 18 \mathrm{~Gy}$, and $21 \mathrm{~Gy}$, respectively, followed by partial mastectomy. Excisions were submitted entirely for histologic review. H\&E stained slides for each case were evaluated for 1) presence or absence of ASM and 2) if present, spatial relationship to the tumor/biopsy site.

Results: This series included 31 cases of postmenopausal women treated with preoperative radiotherapy. Average interval between radiotherapy and excision was 5.9 days (range 3-10.) ASM was identified in 18 cases (58\%), both within the tumor area and away from it. It was more frequent in the highest radiation dose group (13/15) compared to the two lower dose groups (5/16). ASM ranged from focal to diffuse, involving terminal ducts as well as lobules. It typically affected the myoepithelial layer, but a subset of cases demonstrated involvement of luminal epithelium. Importantly, in several cases, ASM mimicked ADH or even DCIS. No ASM was identified in 30 control cases of non-irradiated partial mastectomies.

Conclusions: Atypical squamous metaplasia in the breast is commonly observed in excisions following neoadjuvant radiotherapy. The variable appearance of ASM and its regular presence beyond the tumor bed location creates a diagnostic challenge which may be associated with overestimation of tumor size and incorrect margin assessment, possibly leading to inappropriate additional treatment. Awareness of this phenomenon is important since pre- and peri-operative radiotherapy continue to take on an increased role in treating early breast cancers given the practical appeal of an intact tumor target and the opportunity to improve our understanding of the effect of radiation on breast cancer cells.

126

Heterogeneity within Triple-Negative Breast Cancer with Follow-

Up

AO Adeoye, BJ Youngson, NA Miller, D McCready, SJ Done. University Health Network, Toronto, ON, Canada.

Background: Triple negative breast cancer refers to those breast cancers which are negative forestrogen receptor, progestogen receptor and Her2/neu receptors, thereby unsuitable for hormoneand trastuzumab treatment . It is thought to be a heterogeneous group of breast cancers. They are clinically aggressive and often carry a grim prognosis. Design: We carried out a clinicopathological study of a cohort of triple-negativebreast cancer cases seen in 4 hospitals within the Greater Toronto Area. 192 patients were included in the study based on the selection criteria (i.e. negative for ER, PR, Her2/ neu). Patient biodata, clinical and histopathological information of proven prognostic importance, as well as survival, was retrieved from the records. Tissue microarrays (TMA) slides were constructed from duplicate $0.6 \mathrm{~mm}$ cores of representative tumour tissue and stained for a range of antibodies (9) according to manufacturerrecommended protocols. The TMA slides were scored using the Allred method except for the Ki67 staining which was scored by the visual estimate method of assessing proportion of nuclei in tumoursample taking up the antibody. Results were tabulated and summary statistics was done to determine proportions and statistical significance.

Results: The number of cases in the study was 192 . The age-range is $28-99$ years. The median andmodal age group is $50-59$ years. $70.3 \%$ of the patients are aged between 40 and 69 years. Thetumour size range is $0.6 \mathrm{~cm}$ to $16 \mathrm{~cm} .40 .8 \%$ are pT1, $(<2.0 \mathrm{~cm}), 50.3 \%$ are pT2 $(2-5.0 \mathrm{~cm})$ while $8.9 \%$ are pT3 $(>5.0 \mathrm{~cm})$. The diagnosis is predominantly invasive ductal carcinoma in $84.9 \%$ of patients. Various combinations of other histological diagnosis include metaplastic carcinoma, pleomorphiccarcinoma, invasive lobular carcinoma (ILC), and poorly differentiated carcinoma. CD44 was positive in $17.71 \%$, p63 in $4.17 \%$, p53 in $51.56 \%$, e-cadherin in $81.77 \%$, Vimentin in $77.60 \%$, Cyclin D1 in $22.40 \%$, BCL-2 in $19 \%$, and androgen receptor in $5.73 \%$. $43.75 \%$ of cases showproliferation rates of $\leq 15 \%$, while $26.04 \%$ have proliferation rates of between $5 \%$ and $\leq 14 \% .61 \%$ of patients are alive after 5 years post diagnosis, and were less likely to express the p63 antibody.

Conclusions: This study provides evidence to support the current hypothesis that TNBC are heterogeneous. These mechanisms driving them need to be further investigated. We have presented a clinicopathological and immunohistochemical profile of triplenegative breast cancer which confirms tumor heterogeneity. We advocate for further studies clarify carcinogenic mechanisms driving the tumors in order to further understand them.

127 Determinantes of Axyllary Sentinel Lymph Nodes Positivity: An Analysis of $\mathbf{9 8 5}$ Cases

$S$ AL Diffalha, $P$ Sojitra, $R$ Omman, X Duan, C Godellas, C Perez, S Pambuccian, $R$ Gamez. Loyola University Medical Center, Maywood, IL.

Background: Sentinel lymph node positivity is one of the most important prognostic factor in breast cancer. Numerous studies have showm that tumor size is the most important predictor of lymph node positivity however the impac ER,PR, and Her2 status is less well studied.The aim of this study was to determine the relative contribution of tumor size, grade, and biomarker status (ER, Her2, ki67) on the likelihood of sentinel lymph node positivity.

Design: We performed a retrospective review of all SLNB diagnosed from 1/1/2000 to $5 / 20 / 2013$. We recored age, gender, laterality, and tumor characteristics (diagnosis, tumor size, grade, TNM staging, tumor markers, frozen section and permanent diagnosis on the sentinel lymph nodes. We performed regresion analysis, $\mathrm{X}^{2}$, and non-parametric analysis using SPSS, version 19.0 , with a $\mathrm{p}$ val of $<0.05$ as significant.

Results: A total of 985 patients aged $22-95$ (mean $59.4,-/+12.75$ ) of whom 9 where male and 976 female were included in the study. One to six sentinel lymph nodes totaling 1744 lymph nodes were submitted for histological examination, 246 (25\%) of these patients had at least one sentinel lymph node positive. The mean age was 59.4( $\mathrm{sd}-/+12.75)$, range (22-95). $M=9 \mathrm{~F}=976$. Mutlivariate logistic regresion analysis demostrated that only size (T stage) and Her-2 positivity were the only predictors of sentinel lymph node positivity. However a different regresion model showed that only Her2 poitivity was a significat determinant while size lost its predictor significance. ER, PR, Ki-67 proliferation rate, grade and age had no significant correlation.

Conclusions: This study shows the importance of biomarkers in determining the likehood of lymph node positivity and suggest that a model can be constructed to determine patients with very low likehood of sentinel lymph node metastasis in whoom sentinel lymph node biopsy could be avoided.

128 Frozen Section Diagnosis of Breast Cancer: Discrepancy Rate, and Number of Lymph Nodes and Optimal Number of Sentinel Lymph Node Examined

SAL Diffalha, P Sojitra, X Duan, C Godellas, C Perez, R Gamez, S Pambuccian. Loyola University Medical Center, Maywood, IL.

Background: Frozen section (FS) examination of the sentinel lymph nodes (SLN) is widely used as a method to determine the need for completion of axillary lymphadenectomy. The performance of FS examination of frozen section varies depending on the method of sectioning and the addition of cytologic examination. The optimal number of lymph nodes submitted for frozen section has not been determined. The aim of the study was to determine the performance characteristics of FS examination of SLN in patients with breast cancer and determine the optimal number of SLN to be examined.

Design: Retrospective analysis of SLN biopsies in patients with breast cancer diagnosed between 2000 -2012 was performed and patients demographic, tumor type, size, grade, ER, PR, Her2, and Ki67 status were entered into a spreadsheet.

Results: A total a 985 patients aged 22-91 (mean: $59+/$ - 12.7) underwent SLN biopsies during the study period. 902 patients had FS of 1-6 SLN for a total of 788 frozen sections. On final analysis SLN was positive in 249/1758 (15\%); SLN1 in 146/902 (18\%); SLN2 63/543 second (11.6\%); SLN3 in 18/231 (7.8\%) and the SLN4-SLN6 4/78 (5\%) When SLN1 was negative SLN2-SLN6 was positive in additional 28 patients when SLN1 and SLN2 were negative SLN3 was positive in 5 cases, while if all SLN1, 2 and 3 were negative none of the further SLN were positive. Discrepancies between FS and permanent sections were seen in 75/1758 (4.3\%) cases; all were caused by false negative FS diagnoses. They were more common in the SNL1 (5.4\%), and less common in SLN2, SLN3, and SLN4+ $(3.5 \% ; 2.6 \%$ and $1.3 \%$ respectively). The discrepancy rate was higher in lobular carcinoma 9/59 (15.3\%) than in ductal carcinoma 53/709 (7.5\%) but the difference did not reach statistical significance $(\mathrm{p}=0.06)$. When SLN1 was negative, SLN2 was positive in $28 / 543(5.2 \%)$, and SLN3 was positive in $5 / 231$ cases $(2.2 \%)$. None of the 78 FS of SLN4-6 were positive in the absence of a prior positive SLN.

Conclusions: Our study shows that frozen section/permeant discrepancy rate for sentinel lymph nodes are relatively small but can occur in the 1,2 3 and 4 SLN. Submission of more tan 3 SLN for frozen section analysis does not increase the yield of positive diagnosis and could be safely forgone.

\section{HER2 Positive Rates Vary by County and Geographic Region in} California Independent of Stage and Age at Presentation

KH Allison, KC Jensen, TC Dur, SL Gomez, RB West, AW Kurian. Stanford University School of Medicine, Stanford, CA; Cancer Prevention Institute of California, Fremont, CA.

Background: It is unclear how much regional variation there is for the percent of breast cancers that are HER2 positive. The contribution of regional population characteristics and laboratory practices may influence these rates. Establishing valid laboratory benchmarks may require consideration of these factors.

Design: Utilizing data from 2006-2010 from the California Cancer Registry (CCR), we examined the HER2 status of breast cancers by 58 California counties. Counties were grouped into 10 geographic regions for analysis of stage and age at presentation in additional to HER2 status.

Results: 97,248 breast cancer cases were included in the CCR data. Overall, the HER2 positive rate was $16.5 \%$. The percent HER2 positive cancers in the 58 counties ranged from $11.3-32.3 \%$ with highest variability in the smallest counties. When grouped into 10 regions, the percent positive ranged from 13.2\% (Region 4: San Luis Obispo, Santa Barbara and Ventura Counties) to 18.9\% (Region 9:Los Angeles). The percent positive cases increased with increasing stage at presentation as follows: $12.9 \%$ for stage 1 , $17.4 \%$ for stage $2,23.2 \%$ for stage $3,25.9 \%$ for stage 4 and $25.2 \%$ for unstaged cases. It also varied by age with $14.5 \%$ of cases in women over 55 years old HER 2 positive compared to $21.0 \%$ of cases in women $<55$ years old. When age and stage groupings were combined, women $>55$ years old with early stage (stage 1-2) cancers had the lowest percent HER2 positive (12.8\%), while women in the same age range with later stage (3-4) disease were $22.9 \%$ positive. Women $<55$ with high stage (3-4) disease had the highest positive rates $(27.9 \%)$ and women in the same age group with early stage disease had an $18.9 \%$ positive rate. Variation by geographic region still existed when looking within age and stage groupings (see table). 


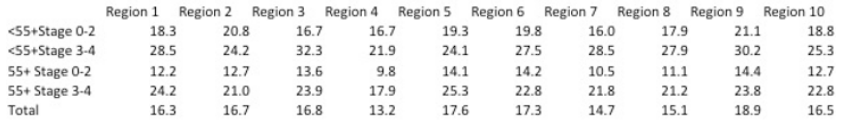

Conclusions: In California, there is significant variation by geographic region in the percent of breast cancers that are HER2 positive, even when controlling for stage and age. The possible influence of differences in regional population characteristics versus laboratory practices to explain this variation should be further examined so that valid regional laboratory benchmarks can be established.

130 Quantitative Gene Expression by RT-PCR in Classic and Variant Lobular Carcinoma in ER+ Breast Cancer

JMAnderson, C Yoshizawa, D Watson, V Tan, HBailey, AC Tsiatis, S Shak, FL Baehner. Genomic Health, Inc, Redwood City, CA.

Background: Classic lobular carcinoma is characterized by a distinctive morphology, loss of E-cadherin commonly due to mutation or deletion of CDH1 on chromosome 16q, and a variable clinical course (Pestalozzi JCO 2008 26:3006). Variants (pleomorphic, solid \& alveolar) with distinct morphologies and potential differences in outcome have been described (Rosen 2009). We provide an 8 -year update of the patterns of quantitative gene expression as measured by the 21 gene Oncotype DX ${ }^{\circledR}$ assay for ductal NOS (DC) and classic and variant lobular carcinomas.

Design: All tumors analyzed in the Genomic Health laboratory from 6/04-5/12 were included. Central path used WHO criteria for classification of classic lobular (CL), solid \& alveolar lobular (SAL), and pleomorphic lobular (PL) carcinomas. Quantitative expression of 16 cancer related genes was measured on a scale from 2 to 15 (relative to reference genes) where a 1 unit increment is associated with an $\sim 2$-fold change in expression. Descriptive statistics for RS \& individual genes [ER, PR, invasion gene group (IGG) \& proliferation gene group (PGG)] among the different subtypes were obtained. Comparisons of means among the subtypes were adjusted to control the overall error rate under any complete or partial null hypothesis.

Results: DC accounted for $81.8 \%$ of 286,726 cases, CL $7.3 \%$, SAL $0.4 \%$ \& PL $0.4 \%$. For all types a continuous range of RS was observed. DC had the greatest percentage of high risk RS followed by SAL, PL \& CL. DC had the highest mean RS and PL \& CL had the lowest RS. SAL had the highest mean ER expression and CL \& PL had the lowest ER expression. These results may reflect a submission bias and are not population based. The proportion with ER+/PR- phenotype was slightly different among the subtypes: SAL $(24.0 \%)$ and PL $(19.4 \%)$ had a higher incidence compared to DC $(14.1 \%)$ and CL (15.2\%). SAL had the highest PGG expression; CL had the lowest. DC had the highest IGG; CL had the lowest. DC had younger patient age than CL, PL and SAL. Conclusions: Classic lobular carcinoma and variants are characterized by differential patterns of gene expression. Outlier cases are not infrequent within each of the subtypes in this large observational cohort. The variation in gene expression, noted by histologic subtype, will be presented in detail.

\begin{tabular}{|c|c|c|c|c|c|}
\hline & & RS Group & & & \\
\hline Histologic Subtype & $\mathrm{N}$ & Low $(<18)$ & Intermediate (18-30) & $\operatorname{High}(\geq 31)$ & $\overline{\mathrm{ER}+/ \mathrm{PR}-}$ \\
\hline Classic Lobular (CL) & 20,844 & $60.8 \%$ & $35.9 \%$ & $3.3 \%$ & $15.2 \%$ \\
\hline Pleomorphic Lobular (PL) & 1,867 & $52.5 \%$ & $39.8 \%$ & $7.7 \%$ & $19.4 \%$ \\
\hline Solid/Alveolar Lobular (SAL) & 1,231 & $58.0 \%$ & $31.5 \%$ & $10.5 \%$ & $24.0 \%$ \\
\hline All Lobular & 23,942 & $60.0 \%$ & $36.0 \%$ & $4.0 \%$ & $16.0 \%$ \\
\hline Ductal NOS (DC) & 234,518 & $53.8 \%$ & $33.3 \%$ & $13.0 \%$ & $14.1 \%$ \\
\hline
\end{tabular}

Independent Predictors of Survival in Male Breast Cancer: Unfavourable Impact of Older Age in a Multivariate Analysis of 166 Patients $S$ Andre, T Pereira, F Silva, P Machado, M Aparicio, GL Silva, AE Pinto. Instituto Português de Oncologia de Lisboa Francisco Gentil, E.P.E., Lisboa, Portugal; FCMUniversidade Nova de Lisboa, Lisboa, Portugal; Universidade de Lisboa, Lisboa, Portugal; Instituto Superior Técnico da Universidade Técnica de Lisboa, Lisboa, Portugal.

Background: Male breast carcinoma (MBC) is a rare disease with scarce relevant prognostic data. The aim of this study was to investigate the independent prognostic value of clinico-pathological characteristics and molecular markers in relation to overall (OS) and disease-free survival (DFS) in MBC

Design: The study involved 166 men with $\mathrm{MBC}$ and mean follow-up time of 81.9 months (1-396). Age, family history, bilaterality, histological type and grading, tumour size (pT), nodal status ( $\mathrm{pN})$, distant metastasis $(\mathrm{M})$, and disease staging were evaluated. Estrogen (ER) and progesterone receptors (PR) and ERBB2 oncoprotein were assessed by immunohistochemistry. ERBB2 gene amplification was determined by FISH, DNA ploidy by flow cytometry, and $B R C A 2$ mutation by PCR. A Cox regression model was used for statistical analysis of the prognostic variables.

Results: The mean and median age of patients were 64.9 and 66.5 years, comprising 3 groups: $<40(4.2 \%), 40-70(54.8 \%)$, and $\geq 70(41 \%)$ years. Fifty-seven $(33.7 \%)$ patients died of disease and $62(37.3 \%)$ experienced disease recurrence. The 5- and 10-year OS and DFS were $70.1 \%$ and $56.7 \%$ and $62.9 \%$ and $52.5 \%$, respectively. The Kaplan-Meier $(\mathrm{K}-\mathrm{M})$ curves of age, $\mathrm{pT}$, $\mathrm{pN}, \mathrm{M}$, disease stage, ERBB2, ERBB2/ERBB2, and BRCA2 were significantly different for OS, while the K-M curves of $\mathrm{pT}$, $\mathrm{pN}, \mathrm{M}$, disease stage, histological grade and PR showed statistical differences for DFS. In univariate analysis, all the previous variables, with the exception of ERBB2 and BRCA2 (excluded due to few data) for OS, and age, which was added, for DFS, were significantly associated with clinical outcome. In multivariate analysis, older age ( $\geq 70$ years) and advanced disease staging ( $\mathrm{pT}, \mathrm{pN}$, and $\mathrm{M}$ ) for $\mathrm{OS}$, as well as the same previous parameters, together with high grade tumours and lack of PR, for DFS, retained statistically significant association with worse prognosis.
Conclusions: Older age and advanced stage show independent unfavourable prognostic impact in MBC. In addition, high grade of tumour differentiation and lack of PR are predictors of worse DFS.

132 Clinical, Morphological and Immunohistochemical Features of Low Grade Adenosquamous Carcinoma of the Breast

$F$ Andriamampionona Tsitohery, $S$ Croce, M Karanian Philippe, V Velasco, G MacGrogan. CHU-JRA Ampefiloha, Antananarivo, Madagascar; Institut Bergonie, Bordeaux, France.

Background: Low grade adenosquamous carcinoma (LGAC) is a very rare tumour representing less than $0.5 \%$ of all breast carcinomas. Consequently, clinical and pathologic features of this entity are not well known.

Design: 21 cases presenting with features of LGAC as described by Rosen and Ernsberger, either in a pure form or in association with other neoplastic proliferations were retrieved from the files of our hospital. Clinical features and patient follow-up were investigated. All cases were reviewed and their morphologic and immunohistochemical features were determined.

Results: Mean age of patients in the series was 62,9 years (44 to 83), mean pathologic tumor size was $20,3 \mathrm{~mm}(6-38 \mathrm{~mm})$. Axillary lymph node status was determined in 13 cases and was negative. Follow up was available for 11 patients (mean follow up of 46,4 months). Four local recurrences and one pleural metastasis were observed. Morphologically, 15 pure LGAC, 5 cases of mixed LGAC and homologous metaplastic spindle cell carcinoma, and 2 cases of mixed LGAC and heterologous osteochondromatous metaplastic carcinoma were individualized. By immunohistochemical analysis, all cases were triple negative (ER-, PR- and Her2-). Ki67 counts were higher in the myoepithelial $(6,25$ to $17,7 \%)$ and the homologous $(12,5 \%)$ and heterologous stromal (15\%) compared to the luminal (3.2\%)compartments. A complete or incomplete layer of myoepithelial cells (P63+, SMA+, EGFR+, CK5/6+ and CK14+) surrounding glandular or trabecular structures (P63-, SMA-, EGFR+/-, CK5/6+/- and CK14-) was observed. Otherwise, the myofibroblastic cells in these lesions were P63 negative and SMA positive. In the cases of LGAC with an homologous metaplastic spindle cell component, the metaplastic stromal spindle tumor cells displayed a myoepithelial phenotype (P63+, SMA+). Immunohistochemical analysis with P63 and SMA showed a continuum of staining between the myoepithelial cells surrounding the glandular structures, the epidermoid clusters, the trabeculae and the metaplastic homologous or heterologous stromal cells.

Conclusions: LGAC are most often indolent tumors with no associated axillary lymph node metastases but with a tendency to recur locally. Our findings confirm the hypothesis that LGAC are are epi-myoepithelial tumours. Their association with spindle cell carcinoma or osteochondromatous metaplastic carcinomas sharing the same myoepithelial phenotypes indicates a filiation between these different lesions.

133 Re-Excision Rates of Invasive Ductal Carcinoma with Lobular Features Compared to Pure Invasive Ductal Carcinoma and Invasive Lobular Carcinoma

D Arps, J Jorns, J Pang. University of Michigan, Ann Arbor, MI.

Background: Invasive ductal carcinoma with lobular features (IDC-L) is not recognized as a distinct subtype of breast cancer by pathologists. A study at our institution suggested that IDC-L may be a distinct variant of invasive ductal carcinoma (IDC), but has clinicopathological characteristics more similar to invasive lobular carcinoma (ILC) and can be diagnosed on core biopsy with good correlation on resection. We sought to study the re-excision rates of IDC-L compared to IDC and ILC at our institution as patient counseling for additional surgery for margin control may be needed for patients with IDC-L.

Design: 178 cases of IDC-L from 1996-2011 were identified from the pathology database. IDC $(n=684)$ and ILC $(n=250)$ cases were obtained from the tumor registry from 1998-2007 for comparison. Surgical procedure, multifocality, tumor size, and residual invasive carcinoma on re-excision were recorded from the pathology reports. Re-excision rates were calculated by excluding cases with mastectomy as first procedure. Lumpectomies and excisional biopsies were included. Only re-excision for margin control of invasive carcinoma was counted.

Results: Multiple tumor foci were more common in IDC-L $(31 \%, 56 / 178)$ and ILC $(26 \%, 64 / 250)$ than IDC $(7 \%, 51 / 684)$. Frequency of mastectomy as the first surgery was similar for the three groups: IDC-L $(30 \%, 53 / 178)$, IDC $(25 \%, 171 / 684)$, and ILC $(28 \%$, $70 / 250)$ as was completion mastectomies: IDC-L (34\%, 20/58), IDC $28 \%(65 / 234)$, and ILC $(29 \% 36 / 126)$. Table 1 shows the overall re-excision rates and frequency of residual invasive carcinoma on re-excision. Table 2 shows the re-excision rates by tumor size.

\begin{tabular}{|c|c|c|c|}
\hline & IDC-L & IDC & ILC \\
\hline Re-excision rates & $46 \%(58 / 125)$ & $46 \%(234 / 513)$ & $70 \%(126 / 180)$ \\
\hline $\begin{array}{l}\text { Frequency of residual invasive carcinoma at } \\
\text { re-excision }\end{array}$ & $45 \%(26 / 58)$ & $26 \%(60 / 234)$ & $52 \%(66 / 126)$ \\
\hline
\end{tabular}

Table 2. Re-excision rates by tumor size
\begin{tabular}{|l|l||l||l||}
\hline Tumor size $(\mathrm{cm})$ & IDC-L & IDC & ILC \\
\hline$<1$ & $43 \%(12 / 28)$ & $46 \%(71 / 155)$ & $\mid 53 \%(24 / 45)$ \\
\hline $1-2$ & $39 \%(26 / 67)$ & $49 \%(99 / 201)$ & $65 \%(53 / 82)$ \\
\hline $2.1-3$ & $55 \%(12 / 22)$ & $42 \%(49 / 116)$ & $91 \%(30 / 33)$ \\
\hline $3.1-4$ & $100 \%(5 / 5)$ & $32 \%(8 / 25)$ & $100 \%(4 / 4)$ \\
\hline $4.1-5$ & $100 \%(1 / 1)$ & $33 \%(3 / 9)$ & $75 \%(3 / 4)$ \\
\hline$>5$ & $100 \%(2 / 2)$ & $57 \%(4 / 7)$ & $100 \%(12 / 12)$ \\
\hline
\end{tabular}


Conclusions: Overall, re-excision rates for IDC-L and IDC were similar and lower than that for ILC. However when stratified by size, re-excision rates for IDC-L and ILC were similar and higher than that for IDC for tumors over $3 \mathrm{~cm}$. Multiple tumor foci were more commonly seen in IDC-L and ILC than IDC, possibly accounting for the greater frequency of residual invasive carcinoma in the re-excision.

\section{4}

High Mobility Group A1 (HMGA1) Proteins in Invasive Lobular Carcinoma

RJ Asch-Kendrick, A Meeker, P Argani, L Resar, A Cimino-Mathews. Johns Hopkins Hospital, Baltimore, MD.

Background: High motility group A1 (HMGA1) is a chromatin remodeling protein important in embryonic development, tumor progression, and cancer stem cell properties. High expression is associated with poor outcomes and markers of advanced disease in diverse tumors, including breast ductal carcinomas. Invasive lobular carcinomas (ILCs) have distinct biologic characteristics compared to ductal carcinomas; they are more likely to occur in older women, to be estrogen receptor (ER) positive, to metastasize late, and to spread to unusual sites. Here, we investigate the role of HMGA1 in breast ILCs.

Design: Tissue microarrays (TMAs) were created from archived paraffin-embedded blocks of 36 primary ILC, confirmed by E-cadherin negativity with full characterization of ER, progesterone receptor (PR) and Her-2 status. HMGA1 protein levels were assessed by immunohistochemistry. Nuclear HMGA1 labeling was scored as intensity and percentage of positive cells. An immunoreactivity score (IR) was the product of staining intensity (weak $=1$, moderate $=2$, strong $=3$ ) and staining extent $(0-25 \%=1$, $26-75 \%=2,>75 \%=3$ ). The average score per case was used for subsequent analyses. Results: We assessed HMGA1 immunoreactivity in 36 ILC cases, including: 34 luminal A phenotype (ER+/PR+/Her2-), 1 luminal B phenotype (ER+/PR+/Her2+), and 1 Her2 phenotype (ER-/PR-/Her2+). Three cases had associated pleomorphic LCIS, and one had histiocytoid features. HMGA1 immunoreactivity was observed in 35 of $36(97 \%)$ cases, with IR ranging from $0-9$ in all cases. Of all cases, the IR was low $(<3)$ in 4 $(11 \%)$, moderate (3-6) in $11(31 \%)$, and high $(>6)$ in $21(58 \%)$. The case with absent HMGA1 staining was luminal A with solid/alveolar morphology, histologic grade II, pathologic stage 1, and had low ki67 staining. The case with metastatic disease (stage 4) and both cases of Her2+ ILC showed high HMGA1 labeling.

Conclusions: This is the first study to evaluate HMGA1 expression in a cohort of ILC with known ER, PR, Her-2 status, stage and histologic grade. HMGA1 immunoreactivity was observed in $97 \%$ of all cases. Although our sample size was limited, the high HMGA1 staining in the metastatic tumor and Her2+ tumors suggest that HMGA1 may be contributing to a more aggressive phenotype associated with these tumors. Further studies are needed to evaluate the role of HMGA1 in Her2 amplification, ER signaling, hormone response, tumor progression in ILCs.

135 Expression Profile of IMP3 in Papillary Lesions of the Breast JR Asirvatham, MB Guzzetta, MD Navarro, TA Bhuiya. Hofstra North Shore-Long Island Jewish School of Medicine, New Hyde Park, NY.

Background: The role of Insulin like growth factor-II mRNA binding protein 3 in breast carcinogenesis is largely unknown, though studies have found weak to intense expression in about a third of breast carcinomas and significant correlation of IMP3 expression with triple negative status, CK 5/6 and aggressive behavior. The aim of this study was to evaluate the expression of IMP3 in papillary lesions of the breast and to determine its utility in diagnosing atypical papillomas.

Design: 64 papillary lesions retrieved from the archives (2007-2013) were selected after confirmation of diagnosis by 2 pathologists and categorized by diagnosis. As terminology for atypical papilloma differs among experts, we designated papillomas involved by a monotonous cell population $<3 \mathrm{~mm}$ as papilloma with ADH and those $>3 \mathrm{~mm}$ as papilloma involved by low grade DCIS. IMP3 was considered positive if granular cytoplasmic staining was observed in $\geq 10 \%$ of the lesion. The proportion and intensity of the stain was scored. HMWK, CK 5/6 and ER expression were also assessed. Fisher exact test was used for statistical analysis.

Results: IMP3 expression was observed in $14 \%$ of benign papillomas, $60 \%$ of papillomas with ADH (figure), $67 \%$ of papillomas with LG-DCIS, $72 \%$ of papillary DCIS, $100 \%$ of solid papillary and invasive papillary carcinomas. The difference in IMP3, CK 5/6 and HMWK expression between benign papillomas and papillomas with $\mathrm{ADH}$ was statistically significant ( $\mathrm{p}=0.009,0.05 \& 0.03$ respectively). Intensity of IMP3 varied from weak to strong in $10-80 \%$ of the lesion.

Immunohistochemical analysis of papillary lesions
\begin{tabular}{|l|l|l|l||l|l||}
\hline Category & $\mathbf{N}$ & IMP3 & CK 5/6 & HMWK & ER \\
\hline Benign Papilloma & 14 & $2(14 \%)$ & $9(64 \%)$ & $10(71 \%)$ & $14(100 \%)$ \\
\hline Papilloma with ADH & 20 & $12(60 \%)$ & $6(30 \%)$ & $7(35 \%)$ & $20(100 \%)$ \\
\hline Papilloma with LG-DCIS & 6 & $4(67 \%)$ & 0 & $4(67 \%)$ & $6(100 \%)$ \\
\hline Papillary DCIS & 11 & $8(72 \%)$ & 0 & $5(45 \%)$ & $10(100 \%)$ \\
\hline Solid and intracystic papillary carcinoma & 8 & $8(100 \%)$ & 0 & $1(13 \%)$ & $7(86 \%)$ \\
\hline Invasive papillary carcinoma & 3 & $3(100 \%)$ & 0 & $1(33 \%)$ & $3(100 \%)$ \\
\hline
\end{tabular}

ary carcinoma

* Loss of tissue precluded assessment in 1 case

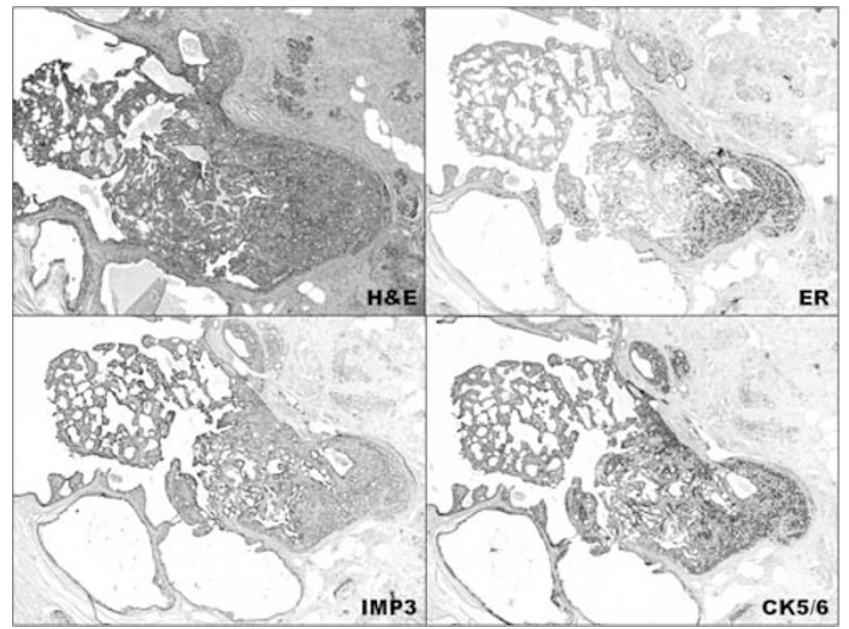

Conclusions: Papillomas with ADH had a significantly higher expression of IMP3 than benign papillomas. The expression of IMP3 by papillomas with ADH was similar to papillomas with LG-DCIS. All solid and invasive papillary carcinomas expressed IMP3. Our study shows that IMP3, in addition to CK 5/6 and HMWK may be a useful test in the evaluation of histologically challenging papillary lesions.

136 Axillary Node Metastases in Breast Cancer: Number, Size, and Extranodal Extension in Sentinel and Non-Sentinel Nodes. A Review of 154 Cases

RM Atkinson, T Park, B O'Hea, P Farrelly, M Lusco, C Tornos. Stony Brook University Medical Center, Stony Brook, NY.

Background: Current NCCN guidelines for breast cancer patients with 1-3 positive axillary nodes state that radiation therapy to the regional lymph node bearing areas should strongly be considered. With the current trend of minimal axillary surgery, complete axillary lymph node dissection (ALND) is not always performed in patients with positive sentinel lymph nodes (SLNs) who undergo breast conservative procedures. The aim of this retrospective study was to assess the risk for additional nodal metastases, more extensive nodal involvement (larger metastases), and extranodal extension in patients with positive SLNs

Design: A search was done to identify all breast cancer patients seen at our institution during a ten year period who underwent SLN biopsy (with positive nodes) followed by axillary node dissection, and for whom all slides were available for examination. In those with positive nodes in the ALND, all slides of lymph nodes were reviewed to assess the total number of positive nodes (SLNs and non-SLNs), size and number of metastases in each category, and presence or absence of extranodal extension in each category. Results: From a total of 154 cases that had positive SLNs followed by ALND, 70 cases $(45.4 \%)$ had additional positive non-SLNs. The number of SLNs ranged from 1 to 7 (median 2). Fifty-eight cases $(82.8 \%$ ) had 3 or fewer SLNs excised. The number of nodes in the ALND (non-SLNs) ranged from 3 to 40 (median 17). The number of positive SLNs ranged from 1 to 6 (median 1). The number of positive non-SLNs ranged from 1 to 39 (median 3). The size of the metastases in the SLNs ranged from $0.2 \mathrm{~mm}$ to $37 \mathrm{~mm}$ (median $10 \mathrm{~mm}$ ). The size of the metastases in the non-SLNs ranged from $0.2 \mathrm{~mm}$ to $39 \mathrm{~mm}$ (median $8 \mathrm{~mm}$ ). The largest metastasis was in a SLN in 39 cases $(55.7 \%)$, and in a non-SLN in 31 cases $(44.3 \%)$. Extranodal extension (EE) was found in the SLN in 39 cases $(55.7 \%)$. Fifteen cases $(21.4 \%)$ showed EE in the non-SLNs without the presence of EE in the SLNs.

Conclusions: In our retrospective revew, almost half the patients were found to have additional positive non-SLNs. The largest metastasis was not always found in a SLN. Additionally, extranodal extension was sometimes present in non-SLNs but absent in SLNs. If the extent of radiation therapy depends on these parameters, patients with 3 or fewer SLNs and no additional ALND may benefit from more extensive regional lymphnodal radiation therapy.

137 Molecular Characterization of Male Breast Cancer by Standardized Quantitative RT-PCR Analysis: A Large Genomic Study of 1,447 Male Breast Cancers

H Bailey, A Tharayanil, JM Anderson, AC Tsiatis, M Bonham, V Tan, M Rothney, FL Baehner, S Shak. Genomic Health, Inc, Redwood City, CA; University of California, San Francisco, San Francisco, CA.

Background: Because male breast cancer is rare ( $<1 \%$ of all breast cancer cases), little is known about the disease and treatment is extrapolated from female breast cancer. Newer molecular technologies have not been used to profile male breast cancer. We report here a study of quantitative gene expression by gender status in tumor specimens submitted for Recurrence Score ${ }^{R}$ (RS) testing.

Design: All invasive breast cancer specimens that were lymph node-negative and ERpositive by RT-PCR successfully examined in the Genomic Health, Inc. laboratory from June 2004 through May 2013 were included. Quantitative expression for each gene was measured by the 21-gene Oncotype $\mathrm{DX}{ }^{\circledR}$ assay on a scale from 2 to 15 (relative to reference genes), where a one-unit increment is associated with an approximate two-fold change in expression.

Results: There were 1,447 (0.52\%) male and 277,940 (99.5\%) female breast cancers. The males were older (mean age 63.1 vs. 57.8 years). A higher percentage of male 
breast cancers were ductal $(85.9 \%$ vs $81.5 \%)$ and papillary $(4.7 \%$ vs $0.6 \%)$. Both male and female breast cancers show a wide variation in gene expression. The distribution of RS in males and females was similar, with mean RS $( \pm \mathrm{SD})$ of $17.4( \pm 11.6)$ in males and $18.0( \pm 11.6)$ in females $(\mathrm{P}=\mathrm{NS})$. The proportion of tumors with RS of $<18,18$ 30 , and $\geq 31$ was $57.2 \%, 29.9 \%$, and $12.9 \%$ in males and $57.7 \%, 33.7 \%$, and $8.7 \%$ in females, respectively. Although the patterns of expression of the genes assessed in the Oncotype DX® assay were more similar than different in males and females, some differences were notable. Mean expressions of ER and PR were 0.4 and 0.5 units higher in males, respectively. Mean expression of the proliferation gene group (Ki-67, MYBL2, Survivin, Cyclin B1, and STK15) was 0.5 units higher in males. The level of quantitative ER increased with increasing patient age in females, however; little increase with age was observed in males.

Conclusions: This large genomic study of male breast cancer reveals a heterogeneous biology as measured by the standardized quantitative Oncotype $\mathrm{DX} ®$ invasive breast cancer assay. While the RS in males and females is similar overall, differences in the hormonal biology were observed. This large genomic study supports the practice of extrapolating treatment of male breast cancer from female breast cancer.

$138 \quad$ Male Breast Cancer: Histological and Clinical Features

D Balakrishnan, C Tornos, J Lu, H Bien, B O'Hea, S Zee, M Singh, J Liu. Stony Brook Medicine, Stony Brook, NY.

Background: Despite advances in prognostic factors in female breast carcinoma, data regarding the prevalence and prognosis of molecular markers is limited in male breast cancer. The objective of this study is to evaluate the expression patterns of estrogen receptor (ER), progesterone receptor (PR), human epidermal growth factor receptor-2 (Her-2), and correlate with survival.

Design: All male patients diagnosed with breast cancer at our institution between 2001 and 2013 were identified by searching our laboratory database. Parameters analyzed included: histologic tumor type, grade, ER, PR, Her-2 status, and survival.

Results: Eighteen cases were identified. Three (16.7\%) were needle core biopsies, while the remaining $15(83.3 \%)$ were excisions. Sixteen $(88.9 \%)$ of the 18 cases were invasive ductal carcinomas, one case was an intracystic papillary carcinoma, and one case was an invasive cribriform carcinoma. All $18(100 \%)$ cases were ER and PR positive. All 18 $(100 \%)$ cases were Her-2 negative. Of the 15 patients who underwent surgical excision, $4(26 \%)$ had nodal metastases. One patient received chemotherapy, hormone therapy (HT) and radiation therapy (RT). Two patients received HT and RT, while five patients received only HT. Adjuvant therapy for the remaining patients were either unnecessary or status unknown. Follow up information was available in 15 patients ranging from 4 to 120 months. All 15 patients were alive and free of disease.

\begin{tabular}{|c|c|c|c|c|c|c|c|}
\hline Case & ER (\%) & PR (\%) & Her-2 & Histology* & Grade & Stage & $\begin{array}{l}\text { NED** } \\
\text { (months }\end{array}$ \\
\hline 1 & Positive & Positive & Negative & IDC & $\sqrt{2}$ & pT1cN1aMx & \\
\hline 2 & 90 & 90 & Negative & IDC & & & 120 \\
\hline 3 & Positive & Positive & Negative & IDC & $\sqrt{2}$ & pT2N0(i-)Mx & $\sqrt{52}$ \\
\hline 4 & Positive & Positive & Negative & IDC & 3 & & \\
\hline 5 & 90 & 90 & Negative & IDC & 3 & pT1cN0(i-)Mx & $\sqrt{36}$ \\
\hline 6 & 90 & 80 & Negative & IDC & $\sqrt{2}$ & pT2NxMx & \\
\hline 7 & 80 & 30 & Negative & IDC & 2 & & 45 \\
\hline & 95 & 15 & Negative & IDC & & pT1(mic)NxMx & \\
\hline 9 & 90 & 80 & Negative & IDC & $\frac{12}{2}$ & pTicNo(i-)Mx & $\sqrt{37}$ \\
\hline 10 & 95 & 90 & Negative & IDC & 2 & & $\sqrt{38}$ \\
\hline 11 & 90 & 90 & Negative & IDC & $=$ & pT4bN1a(sn)Mx & 31 \\
\hline 12 & 90 & 60 & Negative & IDC & 2 & ypT2N0(i-)(sn)Mx & $\sqrt{14}$ \\
\hline 13 & 100 & 100 & Negative & Cribriform (IDC) & & pTleNo(i-)(sn)Mx & 114 \\
\hline 14 & 95 & 90 & Negative & IDC & 2 & pT2N1Mx & \\
\hline 15 & & 12 & Negative & IDC & 2 & pT2N1aMx & 17 \\
\hline 16 & $\sqrt{95}$ & 995 & Negative & Papillary & 2 & pT2N0(i-)(sn)Mx & 6 \\
\hline 17 & 100 & 100 & & IDC & 2 & pT1cN0(i-)Mx & $\sqrt{4}$ \\
\hline 18 & 99 & 95 & Negative & IDC & 2 & pT2N0(i-)(sn)Mx & $\sqrt{4}$ \\
\hline
\end{tabular}

*IDC- Invasive Ductal Carcinoma **NED-No evidence of disease

Conclusions: The unanimous expression of hormone receptors in male breast cancer cases highlights a critical role for hormone receptors in male breast carcinoma.

139

Breast Carcinoma, Primary and Brain Metastases: Morphology, Immunophenotype and Novel Markers

$S$ Barak, S Thomas, Q Ahmed, O Hassan, B Alosh, E Abdulfatah, WJ Kupsky, S Bandyopadhyay, R Ali-Fehmi. Wayne State University, Detroit, MI.

Background: Brain metastases (BM) from primary breast carcinoma (PBC) harbor a poor prognosis. Little is known about their morphology and immunophenotype. Growth factor receptors may impact tumor cell growth and metastasis through various signaling pathways. The aim of this study was to assess the histologic features of these metastases and their expression of Her2Neu, EGFR and NTRK1 (neurotrophic tyrosine kinase receptor 1) by immunohistochemistry (IHC).

Design: We identified 17 sets of matched PBC and BM. Patient's age, hormonal and Her2Neu status in PBC were recorded. Histologic grade and type of BM were assessed and compared to the primary tumor. IHC for ER, PR, Her2Neu, EGFR, and NTRK1, was performed and scored according to standard guidelines. Any positivity with NTRK1 was considered positive.

Results: Median age at time of diagnosis of the BM was 47 years. In PBC, 15 invasive ductal carcinomas (IDC) and 2 invasive lobular carcinomas (ILC) were identified. IDC were moderately differentiated (MD) in 5 cases (29\%) and poorly differentiated (PD) in 10(59\%). Overall, 11(65\%) BM cases displayed similar morphology when compared to PBC, whereas 6(35\%) had discordant morphology. Of these, 4 were MD in PBC and PD in BM. Interestingly, 1 case of PD was MD in BM and 1 case of ILC showed micropapillary features in the BM. BC were ER positive in $7 / 15(46 \%)$ cases, PR positive in $6 / 15(40 \%)$, Her2Neu positive in $6 / 15(40 \%) .5(33 \%)$ cases were triple negative. The expression of ER/PR and Her 2 Neu did not change in $8 / 17(47 \%)$ of BM cases. ER changed from positive to negative in $1(6 \%)$ case and PR from positive to negative in $1(6 \%)$ case. $3(17 \%)$ cases gained Her2neu (including one triple negative case) and $3(17 \%)$ cases lost Her2Neu. 4 triple negative cases stayed triple negative. 2 PBC IHC were not available for comparison .Expression of NTRK1 was present in all BM from IDC (15/15), and in 1/2 BM from ILC cases, (overall 94\%). EGFR was positive in 3 IDC (20\%), all triple negative, and one ILC (50\%).

\begin{tabular}{|c|c|c|c|c|c|c|c|}
\hline \multirow[b]{2}{*}{ Histo/grade } & \multirow[t]{2}{*}{ Total n $(\%)$} & \multicolumn{2}{|c|}{ Her2Neu } & \multicolumn{2}{|l|}{ EGFR } & \multicolumn{2}{|c|}{ NTRK1 } \\
\hline & & $\mathrm{Neg}$ & Pos & $\mathrm{Neg}$ & Pos & Neg & Pos \\
\hline IDC MD & $5(29)$ & $2(40)$ & $3(60)$ & $5(100)$ & 0 & 0 & $5(100)$ \\
\hline IDC PD & $10(59)$ & $8(80)$ & $2(20)$ & $7(70)$ & $3(30)$ & 0 & $10(100)$ \\
\hline$\overline{I L C C}$ & $2(12)$ & $1(50)$ & $1(50)$ & $1(50)$ & $1(50)$ & $1(50)$ & $1(50)$ \\
\hline
\end{tabular}

Conclusions: Most BM from PBC arise from PD IDC. Overall breast carcinomas keep their morphology or become poorly differentiated. Although the immunophenotype did not differ between PBC and BM, the high expression of NTRK1 in BM may offer a potential therapeutic target. Further studies are needed to explore the expression of EGFR in triple negative PBC.

140 Classified on Core Needle Biopsies?

S Barak, S Bandyopadhyay, D Shi, R Beydoun, H Arabi, H Guan, Q Ahmed, S Madan, D Visscher, R Ali-Fehmi. Wayne State University, Detroit, MI; King of Abdulaziz Medical City, Jeddah, Saudi Arabia; Mayo Clinic, Rochester, MN

Background: Fibroepithelial lesions (FEL) of breast are notoriously difficult to classify on core needle biopsies (CNB). The goal of this study was to evaluate interobserver variability and accuracy of sub-classifying difficult fibroepithelial lesions into cellular fibroadenoma (CFA), fibroadenoma (FA), and phyllodes tumors (PT).

Design: We identified 50 breast CNB, initially diagnosed generically as FEL, with subsequent excision and final diagnosis of CFA, FA and PT. 5 surgical pathologists from one institution independently reviewed these in 2 rounds. The pathologists were blinded to final diagnosis. Only age of patients was provided. 3 diagnostic categories were allowed: CFA, FA and PT. Subsequently, pathologists were given a set of histologic criteria including presence of subepithelial condensation, stromal heterogeneity, overgrowth, pleomorphism, and increased cellularity, and more than 2 mitotic figures/10HPF and asked to review the slides for a second round. Interobserver agreement was evaluated using $\kappa$ level of agreement. Accuracy of ratings to final diagnosis was calculated using proper statistical analysis.

Results: $\kappa$ values were fair for first and second rounds varying from 0.20 to 0.22 respectively. When considering each category, $\kappa$ value varied from 0.11 to 0.14 for CFA, 0.26 to 0.29 for FA and 0.28 to 0.14 for PT. Overall there was fair agreement between the pathologists in all categories. The rate of correctly diagnosed cases ranged from $28 \%$ to $50 \%$ in the first round, and from 38 to $70 \%$ in the second round. Overall the pathologists performed better in identifying FA than CFA and PT.

\begin{tabular}{|c|c|c|c|}
\hline Pathologist & Category N & Round 1 N(\%) & Round 2 N(\%) \\
\hline \multirow[t]{3}{*}{$\mathrm{A}$} & CFA3 & $1(33)$ & $1(33)$ \\
\hline & FA 33 & $14(42)$ & $15(45)$ \\
\hline & PT 14 & $3(21)$ & $4(29)$ \\
\hline \multirow[t]{3}{*}{$\mathrm{B}$} & CFA & $3(100)$ & $2(67)$ \\
\hline & FA & $18(55)$ & $18(55)$ \\
\hline & PT & 0 & $3(21)$ \\
\hline \multirow[t]{3}{*}{ C } & CFA & $2(67)$ & $3(100)$ \\
\hline & FA & $15(45)$ & $11(33)$ \\
\hline & $\mathrm{PT}$ & $6(43)$ & $5(36)$ \\
\hline \multirow[t]{3}{*}{$\mathrm{D}$} & CFA & $1(33)$ & 0 \\
\hline & FA & $19(58)$ & $19(58)$ \\
\hline & PT & $5(36)$ & $4(29)$ \\
\hline \multirow[t]{3}{*}{ E } & CFA & $2(67)$ & $1(33)$ \\
\hline & FA & $9(27)$ & $27(82)$ \\
\hline & $\mathrm{PT}$ & $3(21)$ & $7(50)$ \\
\hline
\end{tabular}

The accuracy was significantly different between first (40\%) and second rounds (48\%) $(\mathrm{p}=0.028)$. In the first round, $12(24 \%)$ cases were not correctly diagnosed by any of the pathologist. This number dropped to $6(12 \%)$ in the second round.

Conclusions: Our results highlight the difficulties in distinguishing CFA from PT on CNB. Using standardized criteria may improve the accuracy of diagnosis. In a daily practice, use of indeterminate terms such as FEL as a diagnostic category remains a useful option.

141 GATA-3 Is Expressed More Frequently in Triple Negative Breast Cancer Than the Combination of GCDFP and Mammaglobin

EM Bashover, PE Bomeisl, HL Gilmore, RM Elliott. University Hospitals Case Medical Center, Cleveland, $\mathrm{OH}$

Background: The combination of gross cystic disease fluid protein-15 (GCDFP) and mammaglobin (MGB) has been shown to have high sensitivity and specificity in determining breast origin for metastatic carcinomas. However, "triple" (ER-, PR-, HER2-) negative breast carcinomas (TNBC) have been shown to have lower rates of positivity for these markers than other types of breast cancer. GATA-3 plays a role in breast luminal cell differentiation and has been shown to be involved in breast cancer progression. GATA-3 has been shown to be highly sensitive in studies with a heterogeneous breast carcinoma population. Yet, GATA-3 expression in a large cohort of TNBCs has not been well studied. Our aim was to characterize GATA-3 expression and TNBC and compare its sensitivity with the combination of GCDFP and MGB

Design: A microarray containing TNBC samples from 56 patients was used to prepare 4 micron-thick sections. IHC for GATA-3 (clone L50-823) was performed using a BondMax Automated Immunostainer (Leica) and IHC for GCDFP (clone EP1582Y) and MGB (clone 31A5) was performed using BenchMark Ultra (Ventana Medical 
Systems). All antibodies were obtained from Cell Marque, Rockland, CA. The arrays were then scored as negative, low positive $(<10 \%$ cells staining), and high positive ( $>10 \%$ cells staining)

Results: Of the 56 TNBC samples, 5 were excluded from analysis due to technical failure. Of the remaining 51, $15(29.4 \%)$ were positive for GCDFP-15, 18 (35\%) were positive for MGB, 36 (71\%) were positive for GATA-3, 28 (55\%) were positive for either GCDFP or MGB, $10(25 \%)$ were GATA-3 positive and GCDFP and MGB negative, 7 (14\%) were GCDFP or MGB positive and GATA-3 negative, and (38) 75\% were positive for GCDFP, MGB, or GATA-3. All GCDFP and MGB positive cases and $95 \%$ of GATA-3 positive samples were strong positives.

Immunohistochemical Staining of GCDFP-15, MGB, and GATA3 in Triple Negative Breast Carcinoma

\begin{tabular}{|l|l||l||}
\hline Staining Profile & $\begin{array}{l}\text { lumber of Samples Staining } \\
(\mathrm{n}=51)\end{array}$ & Percent \\
\hline GCDFP-15 + & 15 & 29 \\
\hline MGB + & 18 & 35 \\
\hline GATA-3 + & 36 & 71 \\
\hline GCDFP-15 and/or MGB - & 28 & 55 \\
\hline GATA-3+ and GCDPF-/MGB- & 10 & 25 \\
\hline GCDFP or MGP + and GATA-3- & 7 & 14 \\
\hline GCDFP or MGB or GATA3 + & 38 & 75 \\
\hline
\end{tabular}

Conclusions: GATA-3 stains a large proportion of TNBCs overall. GATA-3 is expressed more frequently in triple negative breast cancer than the combination of GCDFP and mammaglobin combined. In addition, GATA-3 stains a subset of TNBC that are GCDPF-/MGB-, so that the addition of GATA-3 to a panel of stains for breast origin increases overall sensitivity to $75 \%$.

142

PPP2R2A Expression Identifies a Subgroup of Luminal Breast Cancer with Increased Risk of Relapse and Death

F Beca, D Martins, F Schmitt. Dana-Farber Cancer Institute/Harvard Medical School, Boston, MA; IPATIMUP - Institute of Molecular Pathology and Immunology of the University of Porto, Porto, Portugal; Faculty of Medicine, University of Toronto and University Health Network, Toronto, ON, Canada.

Background: PPP2R2A, is a B-regulatory subunit of the PP2A mitotic exit holoenzyme complex. Somatic mutations in PPP2R1A have recently been reported in clear cell and endometriod ovarian cancers. In breast cancer, it was observed a subset of ER positive tumors with loss of transcript expression in PPP2R2A, constituting one of the hallmarks of the Integrative Cluster 2 (IntClust 2), a ER positive group with poor overall and disease-free survival (Curtis C et al., Nature; 2012). Therefore, we aimed to investigate if PPP2R2A expression, evaluated by immunohistochemistry (IHC), could define a group of luminal breast cancer with worse prognosis.

Design: We evaluated the PPP2R2A IHC expression in a total of 807 breast cancer patients from two independent cohorts: discovery cohort $\mathrm{n}=349$ and validation cohort $\mathrm{n}=458$ that included 282 and 337 luminal breast cancers, respectively. PPP2R2A expression scoring was dichotomized and evaluated as a predictor of disease-free survival (DFS) and overall survival (OS) in both cohorts.

Results: Approximately $60 \%$ of luminal breast cancers cases demonstrated PPP2R2A IHC expression (discovery cohort $=58.7 \%$; validation cohort $=57.6 \%$ ). More importantly, loss of PPP2R2A expression identified patients with both shorter disease-free survival (Log-rank; P-value=0.002) with an HR of $0.38(0.202-0.720)$ and overall survival (Log-rank; P-value $=0.001$ ) with an HR of 0.295 (0.135-0.645). The scoring method for PPP2R2A was further validated and remained significantly associated with both diseasefree survival and overall survival in the validation cohort (Log-rank; P-value $=0.031$ and 0.027 , respectively).

Conclusions: The evaluation of PPP2R2A expression by IHC identifies a subgroup of luminal breast cancer patients with high risk of relapse and death and could be potentially used as a surrogate marker for the identification of IntClust 2-like breast cancers.

143 Predictive Values of 16 Micro Rna's Expression Levels in Triple Negative Breast Carcinomas for Lypmh Node Metastasis

U Berber, I Yilmaz, G Narli, A Haholu, Z Kucukodaci, D Demirel. GATA Haydarpasa Training Hospital, Istanbul, Turkey.

Background: We examined expression profiles of sixteen micro RNAs (miRNAs) in triple negative breast cancer cases to identify their potential as biomarkers for lymph node metastasis. Analyzed miRNAs were selected according to their functions in cell cycle, roles on tumor initiation and progression, or their proposed relations with aggressive behavior or metastasis in breast cancer patients.

Design: The expressions of miR-9, miR-21, miR-30a, miR-30d, miR-31, miR-34a, miR-34c, miR-100, miR-122, miR-125b, miR-146a, miR-146b, miR-155, miR-181a, miR-200c, and miR-205 were examined by real-time quantitative reverse transcriptasePCR using SYBR Green method and expression levels were calculated by $\Delta \Delta C t$ method in 32 tumor samples and corresponding benign breast tissues. Differences of miRNA expression levels in tumor samples normalized to benign breast tissues were analyzed in metastatic $(\mathrm{n}=16)$ and non-metastatic $(\mathrm{n}=16)$ patient groups.

Results: In the tumor tissues, seven of the miRNAs (miR-31, miR-205, miR-34a, miR146a, miR-125b, miR-34c, and, miR181a) were down-regulated more than 1.5 fold, whereas, only miR-21 was found to be up-regulated more than 1.5 fold when compared to the benign breast tissues. MiR-31 was the most down-regulated miRNA (mean: $0.247 \pm 0.299$ ) followed by miR-205 (mean: $0.367 \pm 0.353$ ) and miR-34a (mean: $0.396 \pm 0.303$ ). The reduced expressions of miR-205 and miR-200c were significantly associated with lymph node metastasis $(\mathrm{p}=0.016$, and $\mathrm{p}=0.021$, respectively). MiR$200 \mathrm{c}$ levels were decreased in the metastatic group (mean:0.591 \pm 0.531 ), whereas it was slightly increased in non-metastatic group (mean:1.202 \pm 1.009 ). MiR-122 levels were also decreased in metastatic cases, although not significant $(\mathrm{p}=0.051)$. We did not observe any significant association between miRNA expression levels and histological type, histological grade, nuclear grade or tumor diameter.

Conclusions: Our results demonstrated the dysregulation in expression levels of eight miRNAs in triple negative breast cancers. We also found two candidate miRNAs associated with lymph node metastasis (miR-205 and miR-200c). Additionally, a possible association was also indicated with decreased expression levels of miR-122 and metastasis in triple negative breast cancer patients.

144 Microscopic and Immunohistochemical Assessment of Cellular Proliferation and Mitotic Activity in Resection Specimens of Invasive Breast Cancer

A Biernacka, S Dhamne, S Herrera, C Gutierrez, A Contreras. Baylor College of Medicine, Houston, TX.

Background: The proliferative profile of invasive breast carcinomas (IBC) is increasingly becoming more important in determining patient prognosis and to measure responsiveness to neoadjuvant therapy. Historically, Ki-67 has been the marker to study proliferation in these tumors. As histone $\mathrm{H} 1$ is phosphorylated from late $\mathrm{G} 1$ through mitosis and histone $\mathrm{H} 3$ is phosphorylated from late $\mathrm{G} 2$ through mitosis, the addition of these markers to better profile the proliferative potential of carcinomas will aid in better understanding tumor biology. The objective of this pilot study was to examine the expression of phospho-histone H1 (P-H1) and H3 (P-H3) in IBCs by IHC. We aimed to compare $\mathrm{P}-\mathrm{H} 3$ labeling with routine $\mathrm{H} \& \mathrm{E}$ mitotic count and investigate if mitotic index (MI) derived from P-H3 IHC differentially impact histological grade (HG). Since both Ki-67 antigen and phosphorylation of $\mathrm{H} 1$ are detected in both interphase and mitosis, their levels were compared.

Design: Tissue microarray (TMA) was constructed from surgical excisions of 41 IBCs. MI was determined on H\&E sections per $10 \mathrm{HPF}$. Proliferative activity was further studied by IHC for MIB-1/Ki-67, P-H1 (hyperphosphorylated isoform), P-H3 (Ser10) and expressed as percentage or number of positive cells per $10 \mathrm{HPF}$, respectively. HG was established with ESBR criteria. Assessment of MI, HG and IHC was performed jointly by 3 observers under a multi-headed microscope after reaching the consensus. Pearson's and Spearman's rank correlation, univariate linear regression and concordance analysis were used for statistics.

Results: Out of 41 tumors in our TMA, 12 samples were excluded from analysis as they showed similar absent to decreased staining for both $\mathrm{P}-\mathrm{H} 1$ and $\mathrm{P}-\mathrm{H} 3$, suggesting preanalytic variables effecting staining of these phospho antibodies. Out of the remaining 31 tumors, 28 samples showed a correlation with no change in HG between manual mitotic count using H\&E stained section and P-H3 staining per $10 \mathrm{HPF}(\mathrm{p}<0.05)$. Levels of P-H1 correlated with Ki-67 levels in high-grade carcinomas, but the labeling indices were lower for P-H1 than Ki67 $(\mathrm{p}<0.05)$.

Conclusions: Levels of P-H3 correlate with manual mitotic count. P-H3 may be a useful marker to help in grading those carcinomas with apoptotic cells that may be confused with mitotic figures. Levels of P-H1 correlate with Ki-67 in high-grade carcinomas but are consistently lower. P-H1 may enable to identify aggressive lesions without overestimating proliferation by cells arrested at G1/S checkpoint still detected with Ki67.

\section{Impact of Ki-67 Proliferation Index Reporting on Oncotype Dx®} Breast Cancer Assay Referrals: The Mayo Clinic Experience

MC Bois, JM Carter, DM Minot, JS Voss, T Mounajjed, B Chen. Mayo Clinic, Rochester, MN.

Background: Oncotype DX ${ }^{\circledR}$ breast cancer assay has been used for predicting distant recurrence and chemotherapy benefit in patients with ER+/HER2- breast cancer. Proliferative activity of the tumor is a key factor in calculating the Recurrence Score $\mathbb{R}$ (RS). We studied the impact of Ki-67 proliferation index (Ki-67 PI) reporting on the selection of tumors referred for Oncotype DX ${ }^{\circledR}$ breast cancer assay.

Design: Since October 2011, Ki-67 PI has been reported on all invasive breast carcinomas treated at Mayo Clinic Rochester. Ki-67 PI is performed immunohistochemically using the anti-Ki-67 MIB-1 clone and reported as \% of positive-staining invasive tumor nuclei with the aid of digital image analysis using Aperio instrumentation. We performed a retrospective review of all breast tumors referred for Oncotype $\mathrm{DX} \AA$ assay during the 12-month periods prior to (pre Ki-67) and following (post Ki-67) implementation of routine Ki-67 reporting. Tumor characteristics, Ki-67 PI, RS, and chemotherapy use were analyzed.

Results: 179 breast carcinomas met the search criteria wherein 101 tumors were referred for Oncotype $\mathrm{DX}{ }^{\circledR}$ assay pre $\mathrm{Ki}-67$ implementation, and 78 tumors were referred post Ki-67 implementation. The RS median ( 16 vs 16$)$, RS range (0-50 vs 5-56), and median tumor size $(1.7 \mathrm{~cm}$ vs $2.0 \mathrm{~cm}$ ) were similar for the 2 periods. The overall nodal metastatic rates were $40 \%$ and $27 \%$, respectively. Chemotherapy was recommended to $26 \%$ and $18 \%$ of patients, respectively. Tumor characteristics are presented in Table 1. Examples of Oncotype DX ${ }^{\circledR}$ referral scenarios will be discussed.

Tumor Characteristics

\begin{tabular}{|c|c|c|c|c|c|c|}
\hline & \multicolumn{3}{|l|}{ Pre Ki-67 } & \multicolumn{3}{|l|}{ Post Ki-67 } \\
\hline & NG I & NG II & NG III & NG I & NG II & NG III \\
\hline $\begin{array}{l}\text { Number of tumors } \\
(\%)\end{array}$ & $28(28 \%)$ & $69(68 \%)$ & $4(4 \%)$ & $24(31 \%)$ & $46(59 \%)$ & $8(10 \%)$ \\
\hline RS median (range) & $13(1-29)$ & $17(0-44)$ & $27(21-50)$ & $15(8-33)$ & $15(5-56)$ & $25(9-35)$ \\
\hline $\begin{array}{l}\text { Ki-67\% median } \\
\text { (range) }\end{array}$ & $\mathrm{n} / \mathrm{a}$ & $\mathrm{n} / \mathrm{a}$ & $\mathrm{n} / \mathrm{a}$ & $9(2-46)$ & $14(2-40)$ & $26(10-69)$ \\
\hline $\begin{array}{l}\text { Tumor size }(\mathrm{cm}) \\
\text { median }\end{array}$ & 1.8 & 1.7 & 1.3 & 1.8 & 2.1 & 1.5 \\
\hline LN $(+) \%$ & $46 \%$ & $38 \%$ & $25 \%$ & $38 \%$ & $24 \%$ & $13 \%$ \\
\hline Chemo use \%* & $24 \%(6 / 25)$ & $25 \%(14 / 57)$ & $50 \%(2 / 4)$ & $9 \%(2 / 23)$ & $20 \%(8 / 41)$ & $43 \%(3 / 7)$ \\
\hline
\end{tabular}

NG - Nottingham grade. RS - Oncotype DX Recurrence Score. n/a - not available. * - with available data. 
Conclusions: Implementation of Ki-67 PI reporting altered the breast carcinoma subset referred for Oncotype DX ${ }^{\circledR}$ assay, trending toward higher grade and fewer node-positive tumors, and resulting in fewer chemotherapy recommendations. Overall, the number of tumors referred for Oncotype DX ${ }^{\circledR}$ assay decreased by $23 \%$. This may represent a significant cost saving considering the current list price of $\$ 4380$ for an Oncotype $\mathrm{DX} \circledast$ breast cancer assay.

146 MRI-Detected Benign Papillary and Sclerosing Lesions of the Breast: Microvessel Analysis with Radiologic Correlation

$J$ Bond, R diFlorio-Alexander, V Memoli, $W$ Wells, S Poplack, J Marotti. DartmouthHitchcock Medical Center, Lebanon, NH.

Background: Determining histopathologic concordance of abnormalities undergoing MRI-guided needle biopsy is difficult because most MRI-detected lesions are considered abnormal due to intravenous contrast enhancement, which cannot be confirmed ex vivo. Benign papillary and sclerosing lesions account for an important minority of lesions undergoing MRI-guided needle biopsy.To better understand the histopathologic correlation related to MRI enhancement and the impact of detecting these lesions, we evaluated vascularity with radiologic correlation and review of clinical management. Design: The radiology database was retrospectively reviewed to identify all MRI-guided breast biopsies. All 29 benign papillary or sclerosing lesions from 28 patients were reviewed, and one representative block was stained for CD31 to assess vascularity. CD31 stained slides were digitally scanned at high resolution. Lesional areas were outlined and percent area of CD31 immunostaining was calculated and compared to background non-lesional tissue using automated software. MRI features were reviewed by a breast radiologist.

Results: Between 2005 and 2013, 261 MRI-guided biopsies were obtained, of which 176 $(67 \%)$ were benign. Benign papillary and sclerosing lesions comprised $11 \%$ of biopsies: 26 papillary lesions and 3 radial scars. $76 \%$ of exams were performed to evaluate extent of disease in patients with newly diagnosed breast cancer. Compared to background tissue, benign papillary lesions had increased percent area of CD31 staining (2.06 vs. $1.07, \mathrm{P}=0.002)$, but radial scars did not ( 1.61 vs. $0.83, \mathrm{P}=0.31) .9$ of 29 cases $(31 \%)$ were removed surgically with 5 prompting a separate surgical resection. No additional ductal carcinomas were identified. Most lesions were predominantly enhancing foci $(<5 \mathrm{~mm})$ or masses $<11 \mathrm{~mm}$ and had plateau or washout kinetics; only 7 lesions displayed progressive kinetics and only 4 lesions were $\mathrm{T} 2$ hyperintense.

Conclusions: Increased percentage of vessel area was seen in benign papillary lesions, which may account for MRI related contrast enhancement, but not radial scars. Although MRI features were heterogeneous, most lesions were small enhancing foci or masses with plateau or washout kinetics. Further understanding the etiology of MRI enhancement with histologic correlation may add confidence to histopathologic correlation and help to develop discriminating features, thereby decreasing the number of false positive biopsies and surgeries in patients already diagnosed with breast cancer.

147 Excision?

RA Brown, MR Clay, KC Jensen, RB West, KH Allison. Stanford Hospital and Clinics, Stanford, CA; Palo Alto VAHCS, Palo Alto, CA.

Background: Whether to retest Her2 in excisions for invasive carcinoma of the breast after initial testing on biopsy remains a question. If we could predict which cases were likely to have significantly different Her2 results on excision, selective retesting could be performed. We examined pathologic and clinical features of Her2 concordant and discordant cases to determine if there are features that correlate with Her2 discordance and thus predict the need to retest.

Design: Using a variety of approaches including binary logistic regression, we correlated features of invasive carcinomas found on core or excisional biopsy with Her2 FISH results on biopsy and later excision. Age, nuclear grade, stage, hormone receptors, Ki67\%, lymphovascular invasion, necrosis, calcifications, lymph node involvement, in situ disease, and pre-treatment with chemotherapy were analyzed for 146 cases ( 73 biopsies and 73 matched subsequent excisions).

Results: 7 cases had discordant Her 2 results on excision, while 66 cases were concordant (9.6\% with a change in Her2 status). Of discordant cases, $3(42.9 \%$ ) changed from Her2 negative to positive, and $4(57.1 \%)$ changed from Her2 positive to negative. Discordant cases were enriched for high proliferative index and demonstrated discordance in other prognostic/predictive markers.

\begin{tabular}{|c|c|c|c|c|c|c|c|}
\hline Case & Age & $\begin{array}{l}\text { Her2 FISH } \\
\text { Ratio }\end{array}$ & ER Score, \% & PR Score, $\%$ & Ki67\% & $\begin{array}{l}\text { IDC Grade, } \\
\text { DCIS Grade }\end{array}$ & $\mathrm{Nec} / \mathrm{Calcs}$ \\
\hline $1 \mathrm{~A}$ & 62 & 2.03 & $3+, 95 \%$ & $2+, 70 \%$ & $30 \%$ & $2, \mathrm{~N} / \mathrm{A}$ & $-1-$ \\
\hline 1B & 62 & 1.80 & N/A & N/A & $\mathrm{N} / \mathrm{A}$ & $2, \mathrm{~N} / \mathrm{A}$ & $-1-$ \\
\hline $2 \mathrm{~A}$ & 65 & 3.29 & $0,0 \%$ & $0,0 \%$ & $30 \%$ & $3, \mathrm{~N} / \mathrm{A}$ & $+1 /-$ \\
\hline $2 \mathrm{~B}$ & 66 & 1.98 & $0,0 \%$ & $0,0 \%$ & $5 \%$ & 3,3 & $4+-$ \\
\hline $3 \mathrm{~A}$ & 43 & 2.13 & $0,0 \%$ & $0,0 \%$ & $40 \%$ & 3,3 & -1- \\
\hline $3 \mathrm{~B}$ & 43 & 1.66 & $0,0 \%$ & $0,0 \%$ & $20 \%$ & 3,3 & $+1 /-$ \\
\hline $4 \mathrm{~A}$ & 44 & 1.13 & $3+, 95 \%$ & $2+, 60 \%$ & $60 \%$ & 3,3 & $+1 /-$ \\
\hline $4 \mathrm{~B}$ & 44 & 4.72 & $2+, 25 \%$ & $3+, 15 \%$ & $80 \%$ & 3,3 & $+1+$ \\
\hline $5 \mathrm{~A}$ & 51 & 1.29 & $3+, 95 \%$ & $3+, 95 \%$ & $60 \%$ & 2,3 & $-1-$ \\
\hline $5 \mathrm{~B} *^{\circ} \Delta$ & 53 & 2.09 & N/A & N/A & $\mathrm{N} / \mathrm{A}$ & 2,3 & $+1++$ \\
\hline $6 \mathrm{~A}$ & 74 & 1.05 & $0,0 \%$ & $0,0 \%$ & $40 \%$ & $3, \mathrm{~N} / \mathrm{A}$ & $-1-$ \\
\hline $6 \mathrm{~B}$ & 74 & 3.63 & $0,0 \%$ & $0,0 \%$ & $\mathrm{~N} / \mathrm{A}$ & 3,3 & $+1+$ \\
\hline $7 \mathrm{AA}$ & 45 & 2.00 & $0,0 \%$ & $0,0 \%$ & $50 \%$ & 2,2 & $4+1-$ \\
\hline 7B* & 45 & 1.07 & $1-2+, 5 \%$ & $0,0 \%$ & $40 \%$ & 2,2 & $-1-$ \\
\hline
\end{tabular}

A, biopsy specimen; B, excision specimen; N/A, feature not present for evaluation; +, feature present; -, feature absent. IDC, invasive ductal carcinoma; DCIS, ductal carcinoma in situ; Nec, necrosis; Calcs, calcifications; *treated; ${ }^{\circ}$ positive lymph node. All cases were stage 1 except as designated by $\Delta$ indicating a stage 2 cancer. No case had lymphovascular invasion.
Conclusions: Based on a limited dataset, we identified potential features to predict Her2 discordance. Although larger cohorts are needed to assess the significance of these observations, this approach begins to address the emerging concept of tumor heterogeneity and its clinical significance.

148 of 42 Cases

MDM Capo-Martinez, C Patel, J Liu, S Hoda, B O'Hea, C Tornos. Stony Brook Medicine, Stony Brook, NY

Background: Heterogeneity for Her-2 is well recognized in invasive breast carcinoma. However, heterogeneity for Ki67 is not well documented in the literature. The aim of this study was to assess Ki67 heterogeneity in invasive breast carcinomas.

Design: Immunohistochemistry for $\mathrm{Ki} 67$ was evaluated in large tumor sections from resection specimens (lumpectomies/mastectomies) of 42 invasive breast carcinomas. Heterogeneity was defined as the presence of a low (less than 10\%) and high (more than $30 \%$ ) proliferative activity (PA) within the same tumor in the same histologic section. Results: Three of 42 cases $(7 \%)$ showed heterogeneity as defined above. One was a $1.5 \mathrm{~cm}$ poorly differentiated ductal carcinoma positive for ER, PR and Her- 2 . The PA varied from $5 \%$ to $80 \%$ in different areas of the tumor, with an overall score of $40 \%$. Another case was a $2 \mathrm{~cm}$ well differentiated invasive papillary carcinoma, ER and PR positive and Her- 2 negative, with a PA between less than $1 \%$ and up to $40 \%$, with an overall score of $10 \%$. The third case was a $5 \mathrm{~cm}$ poorly differentiated metaplastic carcinoma, triple negative, with a PA between $5 \%$ and $80 \%$ with an overall score of $70 \%$. Conclusions: Ki67 heterogeneity is unusual but can be seen in some cases of invasive breast carcinoma as demonstrated in $7 \%$ of cases in this series. In these cases, limited sampling in core biopsy specimens may not truly reflect the PA of the entire tumor. If the measured PA with Ki67 appears to be discordant with tumor grade, and the Ki67 value could have an impact on clinical decisions, it may be prudent to repeat the test in large tissue sections from the resection specimen.

149 Quantitative Standardization of HER2 Immunohistochemistry Shows Molecular Heterogeneity of the Protein's Extracellular Domain

DE Carvajal-Hausdorf, KA Schalper, DL Rimm. Yale University, New Haven, CT.

Background: Between 30 and $50 \%$ of patients with HER2 positive breast cancer show no benefit from targeted therapy (trastuzumab). Recent CAP surveys show that 5 commercial antibodies are in common usage for the companion diagnostic immunohistochemistry (IHC) assay (4B5, A0485, CB11, Herceptest, and SP3). All of these antibodies bind the intracellular domain (ICD) of HER2 except SP3 which binds the extracellular domain (ECD). Here we determined the reproducibility, heterogeneity and concordance with FISH for one ECD (SP3) and one ICD (CB11) antibody by quantitative immunofluorescence (QIF).

Design: A custom index tissue microarray (TMA) was built including samples from 80 breast carcinomas with known HER2 status as determined by the Yale Pathology clinical lab (using both IHC and FISH), 10 breast cancer cell lines with known HER2 gene copy number and non-tumor breast tissue. Levels of HER2 protein were measured by QIF using the mouse monoclonal antibody clone CB11 (Biocare Medical) and rabbit monoclonal SP3 (Spring Biosciences).

Results: Both antibodies showed comparable membranous staining patterns although some cases were clearly positive with CB11 and negative for SP3, while the reverse was never seen $\left(\mathrm{R}^{2}=0.69\right)$. The slope $(0.26)$ confirms the visual observation that many cases show substantially lower scores using SP3 than CB11. Reproducibility was assessed for each antibody on serial sections and on sections from 4 separate TMA builds from the identical cases. The serial section reproducibility is $\mathrm{CB} 11 ; \mathrm{R}^{2}=0.97$ and $\mathrm{SP} 3 ; \mathrm{R}^{2}=0.74$. The average correlation as measured by the average regression of each of 4 TMA build compared to all of the others was CB11; $\mathrm{R}^{2}=0.88$ and SP3; $\mathrm{R}^{2}=0.48$ (Mann-Whitney's $P=0.002$ ). The coefficient of variation (CV) of scores was higher for the extracellular target in all studied preparations (SP3 CV $=26.34 \%$ vs CB11 CV $=2.23 \%$ ). When compared to FISH, there are more SP3 negative amplified cases than CB11. CB11 shows more cases that are positive but not amplified.

Conclusions: Although, the concordance between the 2 antibodies targeting the intra and extracellular HER2 domains is good, the ECD signal is more variable, and more often negative than the ICD. This data suggests that measurement of the ECD could be more specific, but the ICD could be more sensitive for prediction of response to therapy. The latter might have profound biological implications since there are both ECD (antibody-based) and ICD (small molecule) therapeutics in the clinic.

150 Invasive Breast Carcinoma with ER-Negative/PgR-Positive Phenotype Has Distinct Clinicopathologic Features

MC Chang, A Ocana, R Goldstein, JJ Cruz-Hernandez, B Lategan, E Amir. Mount Sinai Hospital, Toronto, ON, Canada; University of Toronto, Toronto, ON, Canada; Albacete University Hospital, Albacete, Spain; Mount Sinai Hospital \& University of Toronto, Toronto, ON, Canada; Hospital University de Salamanca, Salamanca, Spain. Background: Hormone receptor (HR) positivity in invasive breast carcinoma has prognostic and predictive significance. Most HR-positive tumors are ER positive. By comparison, tumors that are ER-/PgR+ are uncommon. Some authors question whether this subgroup represents a technical artifact of immunohistochemistry. Some data support the ER-/PgR+ phenotype being a true biologic entity. The aim of this study was to assess for clinicopathologic features of ER-/PgR+ breast cancer in a large cohort of annotated cases.

Design: Databases of consecutive patients diagnosed with invasive breast carcinoma in Salamanca, Spain and in Toronto, Canada were pooled $(\mathrm{N}=816)$. ER, PgR, and 
HER status were reviewed/reported according to ASCO/CAP criteria at both sites. Retrospective chart review was conducted for recurrence and death. Descriptive statistics and Kaplan-Meier survival analysis were applied.

Results: Of the cases analyzed, $623(76.3 \%)$ were ER-positive, $136(16.7 \%)$ were HR-negative (ER-/PgR-), and 57 (7.0\%) were ER-/PgR+. Compared to ER-positive cases, those with ER-/PgR + tumors were younger (median age 50 vs. 57 year, $\mathrm{p}=0.027$ ), less likely to be menopausal ( $32.6 \%$ vs. $49.1 \%, \mathrm{p}=0.012$ ), more likely to be high grade $(50.9 \%$ vs $24.6 \%, \mathrm{p}=0.004)$ and more likely to be HER2-positive $(42.1 \%$ vs. $14.3 \%$, $\mathrm{p}<0.001)$. There was a non-significant association with lymph node metastases $(52.6 \%$ vs. $41.9 \%, \mathrm{p}=0.08)$. There were no differences in median tumor size $(1.8 \mathrm{~cm}$ vs. $2.0 \mathrm{~cm}$, $\mathrm{p}=0.63)$. Compared with HR-negative cases, ER-/PgR+ tumors had smaller median size $(1.8 \mathrm{~cm}$ vs. $2.5 \mathrm{~cm}, \mathrm{p}=0.007)$, had a non-significant association with younger age (median age 50 vs. 58.5 years, $\mathrm{p}=0.26)$, and menopausal status $(32.6 \%$ vs. $34.6 \%$, $\mathrm{p}=0.06)$ and were less likely to be high grade $(76.5 \%$ vs $50.9 \%, \mathrm{p}=0.001)$. There were no differences in HER2-positivity rate ( $42.1 \%$ vs. $43.4 \%, \mathrm{p}=0.87$ ) or likelihood of lymph node metastases $(52.6 \%$ vs. $46.3 \%$, $\mathrm{p}=0.64)$. Among ER-/PgR+ cases, both disease-free and overall survival were intermediate between ER-positive and HR-negative cases. The timing of recurrence more closely resembles HR- negative disease, with an earlier peak, but fewer late recurrences seen in ER-positive cases.

Conclusions: ER-/PgR+ breast carcinoma is an uncommon, but distinct, subgroup with more aggressive phenotype than ER-positive tumors and a pattern of recurrence more similar to HR-negative tumors.

\section{Tumour-Infiltrating Lymphocytes as a Predictor of Response} in Neoadjuvant Endocrine Therapy in Breast Cancer

$N$ Chang, SW Hakim, Z Kos, DH Gravel, A Arnaout, SJ Robertson. University of Ottawa, Ottawa, ON, Canada.

Background: The potential predictive value of tumour-infiltrating lymphocytes (TILs) in breast cancer is recognized in the literature. Studies have focused on TILs in neoadjuvant chemotherapy, which though controversial, have been associated with complete pathologic response and survival. By comparison, few studies exist on TILs in neoadjuvant endocrine therapy (NET). Only changes in T-cell subtypes have been studied in relation to NET response. Here we explore the response of ER+ tumours to short-course estrogen blockade, and whether a raw TIL score, which is more clinically practical than lymphocyte subtype analysis, can predict treatment response (TR).

Design: Patients with strongly ER+ invasive breast cancer on core biopsy were treated with an aromatase inhibitor (anastrozole $1 \mathrm{mg}$ daily) for $14-35$ days $(\mathrm{m}=24.7, \mathrm{sd}=6.7)$ prior to surgical resection. We evaluated TILs in biopsies and resection specimens from 19 NET patients (NETPs) and 23 retrospective controls closely matched for time (4-8 weeks) between biopsy and resection. TILs were scored as follows: 0 (absent), 1 (weak), 2 (moderate), 3 (dense). Ki67 and ER/PR status were reviewed for TR. Pearson correlation coefficients (r) and paired and independent T-tests were performed for statistical analysis.

Results: TILs correlated with Ki67 in biopsies from all cases $(r=0.488, \mathrm{p}<0.001)$, indicating higher raw TIL score is associated with more proliferative tumours. Ki67 significantly decreased between biopsy and resection in NETPs $(\mathrm{m}=-14.6, \mathrm{sd}=17.8$, $\mathrm{p}=0.002$ ), but not in controls, indicating a rapid TR to estrogen blockade in NETPs. PR Allred scores also significantly decreased post-NET (Wilcoxon signed-rank test $Z=-3.41$, $\mathrm{p}<0.001)$. TILs correlated with Ki67 in resections from controls $(\mathrm{r}=0.439, \mathrm{p}<0.02)$, as expected with no intervention, but not in NETPs. No correlation was found between post-NET change in Ki67 and change in TIL or TIL score, indicating TIL does not predict change in Ki67. No significant difference was found in TILs pre- and post-NET. When NETPs were subdivided into responders and non-responders based on $\geq 40 \% \mathrm{Ki} 67$ drop from baseline, no significant difference was found in TILs pre- and post-NET in responders, or in TIL change in responders versus non-responders.

Conclusions: Although short-course NET led to a rapid suppression of proliferation in strongly ER+ tumours, raw TIL score does not predict TR. The lack of change in TILs pre- and post-NET does not preclude alteration of lymphocyte subtypes, for which further study may be warranted.

152 Oncotype DX in ER Positive Breast Cancer with isolated Tumor Cells (pN0i) or Micro Metastases (pN1mi) in Lymph Node

$S$ Chaudhary, A Nayak, M Hanna, C Nagi, I Bleiweiss, S Jaffer. Mount Sinai Medical Center and Icahn School of Medicine, New York, NY.

Background: Oncologic management of invasive breast cancer patients with pNOi or pN1mi disease is unclear. Oncotype DX, a 21 gene RT-PCR assay is often used to determine the benefit of chemotherapy in addition to hormonal treatment in ER+, lymph node negative invasive breast carcinoma. However, the utility of oncotype DX testing in $\mathrm{ER}+$, lymph node positive patients is still somewhat undefined. The available oncotype data to date on such patients is based on the test's results in a cohort of patients from SWOG 8814 trial, a study of ER+, pT1-3a, pN1-2(clinical N0-N1) and M0 invasive breast cancer. Thus, we were interested in examining the relation between oncotype DX recurrence score and clinical-pathologic features in breast cancer patients with pNOi or $\mathrm{pN} 1 \mathrm{mi}$.

Design: We identified patients with $\mathrm{ER}+, \mathrm{pT} 1-2, \mathrm{pN} 0 \mathrm{i} / \mathrm{N} 1 \mathrm{mi}$ that underwent oncotype DX testing from our institution. Cases were categorized into 3 groups based on the recurrence score: low (0-17), intermediate (18-30) and high (>31). Clinical data was recorded, and slides were reviewed to confirm $\mathrm{pNOi} / \mathrm{pN} 1 \mathrm{mi}$ nodal status, type of carcinoma (ductal, lobular or mixed carcinoma), Nottingham grade, morphologic subtype if any, presence of duct carcinoma in situ (DCIS) or lymphovascular invasion, ER, PR and Her2 expression.

Results: The study cohort was comprised of 44 patients ( $25 \mathrm{pNO}$ and $19 \mathrm{pN} 1 \mathrm{mi})$ with age range of 34-81 years (mean=57). The size of the tumors ranged from $0.6 \mathrm{~cm}$ to 3.0 $\mathrm{cm}($ mean $=1.4 \mathrm{~cm})$. Seventeen $(38.6 \%)$ cases $(8 \mathrm{pN} 0 \mathrm{i}, 9 \mathrm{pN} 1 \mathrm{mi})$ were categorized as low, $21(47.7 \%)$ as intermediate $(12 \mathrm{pN} 0 \mathrm{i}, 9 \mathrm{pN} 1 \mathrm{mi})$ and $6(13.6 \%)$ as high recurrence score $(5 \mathrm{pN} 0 \mathrm{i}, 1 \mathrm{pN} 1 \mathrm{mi})$. None of the parameters studied (size, Nottingham grade, ductal, lobular or mixed carcinoma, PR expression, Her2 expression, morphologic subtype, presence of DCIS or lymphovascular invasion, size of isolated tumor cells or micro metastases) correlated with the recurrence score.

Conclusions: Most $(80 \%)$ of the cases had a low to intermediate recurrence score. The oncotype recurrence score did not correlate with any specific histologic or immunohistochemical features, including lymphatic invasion. pNOi or pN1mi patients showed a range of oncotype recurrence scores and did not populate either low or high range exclusively. This suggests that oncotype DX may be of value in guiding treatment in this group of patients.

\section{From In Situ to Invasive Ductal Carcinoma - Are There} Immunophenotypes That Correlate with Disease Progresion?

H Chen, Z Zhang, J Wang, B Wei, J Da, S Hajdu, K Skinner, D Hicks, H Bu, P Tang. University of Rochester Medical Center, Rochester, NY; West China Hospital, Chengdu, China; RTI Health Solution, Research Triangle, NC; China-Japan Friendship Hospital, Beijing, China; Pathology Consultants, Westlake, CA.

Background: Ductal carcinoma in situ (DCIS) is a non-obligatory precursor for invasive ductal carcinoma (IDC); only about $20-40 \%$ of DCIS will progress to IDC. By comparing the expression pattens of a panel of immunohistochemical (IHC) markers between pure DCIS (pDCIS) and DCIS component with co-existing IDC (cDCIS), we intend to identify an immunophenotype in DCIS that could predict an increased risk for progression to IDC.

Design: From consecutive cases of breast carcinomas diagnosed between 1997 and 2008 in our institution, we obtained informative cores from 101 cases of pDCIS and 225 cases of cDCIS from 13 tissue microarray blocks. Clinicopathological information including patient age, tumor size and nuclear grade (NG) was recorded. IHC analyses for estrogen receptor (ER), progesterone receptor (PR), HER2, Ki-67, AR (androgen receptor), IMP3 (Insulin-like growth factor II mRNA-binding protein 3) were performed. Scores were considered positive with $1 \%$ of nuclear staining for ER, PR and AR, 3+ membrane staining for HER2, $>14 \%$ of nuclear staining for Ki-67, and any strong cytoplasm staining for IMP3.

Results: We observed that large tumor size and high NG were significantly associated with pDCIS, and expression of IMP3 was significantly associated with cDCIS. When we compared pDCIS and cDCIS in different subgroups by patient age, tumor size, NG and expression of ER/PR, HER2 and Ki-67, we observed that pDCIS was associated with large tumor size in either the young age or high NG subgroup, with high NG in either ER+/PR+ or Ki-67 14\% subgroup, with positive expression of ER, PR and AR in the high NG subgroup, and with HER2 over-expression in either old age or ER-/PR- or $\mathrm{Ki}-67 \leq 14 \%$ subgroup. We also observed cDCIS was associated with $\mathrm{Ki}-67>14 \%$ in the high NG subgroup and with IMP3 expression in either large tumor or high NG subgroup. Conclusions: We have shown a correlation between expression of IMP3 and Ki-67 and cDCIS and between expression of HER 2 and AR and pDCIS, suggesting that these molecules may play a role in disease progression in subgroups of DCIS. Further study of the immunophenotype of different subgroups of pDCIS and cDCIS is warranted as these markers may be important in understanding the progression of DCIS to IDC, and they may be served as predictive markers for high risk DCIS.

154 The Rate of Upgrade for Various Types of Mammary Atypia in Mammographically Detected Lesions: A Study of 347 Cases from a Single Institution

X Chen, D Wang, X Huang, S Liu, T Khoury. Roswell Park Cancer Institute, Buffalo, NY. Background: It is established that the risk of upgrade is higher in atypical ductal hyperplasia (ADH) than flat epithelial atypia (FEA) or lobular neoplasia (LN) diagnosed on a core needle biopsy (CNB). These lesions frequently co-exist in the same core. However, the rate of upgrade in mixed lesions is unclear.

Design: We reviewed 347 cases with mammary atypia (pure $\mathrm{ADH}=102$, pure $\mathrm{FEA}=34$, pure $\mathrm{LN}=98$, mixed $\mathrm{FEA} / \mathrm{LN}=12$, mixed $\mathrm{ADH} / \mathrm{FEA}=81$, mixed $\mathrm{ADH} / \mathrm{LN}=11$, and mixed $\mathrm{ADH} / \mathrm{FEA} / \mathrm{LN}=28$ ) from RPCI files between 2006 and 20013. The pathologic features of ADH were assessed including number of foci, size of largest focus with complete involvement, and number of involved cores. We recorded the reason for biopsy (mass or microcalcifications). Clinical information including patient age, race, menopausal status, and history of breast cancer (concurrent or past, ipsilateral or contralateral) are also recorded. An upgrade was considered when the subsequent surgical excision specimen yielded invasive carcinoma (IC) or ductal carcinoma in situ (DCIS). Fisher exact test was used for statistical analysis.

Results: Among 347 cases, the upgrade rate was $20.17 \%$. The upgrade rates for pure $\mathrm{ADH}$, pure FEA, pure $\mathrm{LN}$, mixed FEA/LN, mixed ADH/FEA, mixed $\mathrm{ADH} / \mathrm{LN}$, and mixed ADH/FEA/LN was $24.5 \%, 8.8 \%, 10.2 \%, 0 \%, 28.4 \%$ r, $18.2 \%, 25 \%$ respectively $(\mathrm{p}<0.01)$. When $\mathrm{ADH}$ was present (pure or mixed), the risk of upgrade was $28.1 \%$. When pure lesions (ADH, FEA or $\mathrm{LN}$ ) were compared with mixed lesions, the rate of upgrade was also statistically significant $(\mathrm{p}<0.01$ ). Comparing pure $\mathrm{ADH}$ to mixed $\mathrm{ADH}$ with any other additional histologic risk factor (FEA, LN or both); the risk of upgrade was $24.5 \%$ and $26.7 \%$, respectively with no statistically significant difference. The number of foci, size of largest ADH focus and number of involved cores significantly predicted the upgrade. When we controlled these factors in the multivariate analysis, FEA and LN had no additional risk of upgrade for ADH. African American women were at higher risk of upgrade post LN diagnosis on CNB ( 3 of 7) than Caucasian women (7 of 86) $(\mathrm{p}=0.04)$. Mass lesion was an indicator for upgrade for $\mathrm{ADH}$ (mixed or pure) $(\mathrm{p}=0.02)$. Conclusions: Here we outline the risk of upgrade for various types of mammary atypia in mammographically detected lesions. When additional risk factors are included in $\mathrm{ADH}$, the risk of upgrade is not changed. 
155 Solid Papillary Neoplasms with Reverse Polarization (SPNRPs): Histologic, Immunophenotypic and Genotypic Characterization of a Distinctive Breast Tumor

$S$ Chiang, KS Cole, G Baker, K Singh, JM Batten, AJ Iafrate, SJ Schnitt. Memorial Sloan-Kettering Cancer Center, New York, NY; Massachusetts General Hospital, Boston, MA; Beth Israel Deaconess Medical Center, Boston, MA; Montefiore Medical Center, New York, NY.

Background: Since 2005 we have encountered 13 examples of a breast tumor with distinctive morphologic features that we have termed solid papillary neoplasms with reverse polarization (SPNRPs). This report details the clinical, histologic and immunophenotypic features of these tumors and provides the first genotypic analysis of these lesions.

Design: H\&E slides and immunostains for myoepithelial markers, cytokeratins, EMA, ER, PR, HER2 and breast- and thyroid-specific markers were reviewed. Targeted mutational analysis of 14 clinically relevant cancer genes was performed using SNaPshot multiplexed genotyping on 9 cases for which paraffin blocks were available. Pathology reports were abstracted to obtain clinical information.

Results: Patient age ranged from 51-79 years (median 64). Median tumor size was $0.9 \mathrm{~cm}$ (range $0.6-1.4 \mathrm{~cm}$ ). The tumors consisted of circumscribed, solid papillary and less often, papillary nests haphazardly arranged in collagenous stroma, surrounding normal ductal-lobular structures and extending into adipose tissue. Tumor cells were columnar with eosinophilic cytoplasm and low to intermediate grade nuclei. Foamy histiocytes within fibrovascular cores and striking reverse nuclear polarity were noted. Myoepithelial cells were absent in all tumors on p63, calponin and SMMHC stains. There was strong AE1/AE3, CK7, CK5/6 and 34ßE12 expression and patchy EMA and GCDFP-15 staining. Mammaglobin staining was seen in 55\% of cases. Thyroglobulin, TTF-1 and HER-2 were negative in all tumors. ER expression was focally present in $40 \%$ of cases. A missense point mutation in PIK3CA, 3140A $>\mathrm{G}$ (H1047R), was identified by SNaPshot genotyping in 5 of $9(56 \%)$ lesions. No other genetic aberrations including mutations in $B R A F$ were detected.

Conclusions: SPNRPs represent a histologically low grade, invasive neoplasm of the breast with distinctive morphologic, immunophenotypic and genotypic features occurring primarily in older women. SNaPshot genotyping identified a single, recurrent somatic mutation in PIK3CA in over 50\% of these lesions. Although tumors with these features have been previously described as "breast tumors resembling tall cell variant of papillary thyroid carcinoma," absence of thyroid-specific marker expression and BRAF mutations suggest that they are unrelated to papillary thyroid carcinoma.

156 Breast Cancer in Young Adults under the Age of 40 Years: Demographic, Radiologic and Clinicopathologic Variations in Different Ethnic Groups

DA Chitale, A Stark, M Burke, J Abrams, RJ Zarbo, SD Nathanson, F FernandezMadrid. Henry Ford Hospital, Detroit, MI; Wayne State University, Detroit, MI

Background: Breast cancer (BC) in young adults under the age of 40 years with an annual incidence of $7 \%$ is an aggressive disease and comprises a unique group of patients requiring specialized and long term care such as genetic counseling, fertility concerns, maintenance of bone health, secondary prevention etc. The aim of this study was to assess prevalence of clinicopathologic characteristics in a group of young adults under the age of 40 years who were referred for imaging study because of diagnostic mammograms (palpation of a lump, tenderness and/or nipple discharge) and screening mammograms. Design: We studied a sample of 443 patients less than 40 years with mammographic diagnosis of BIRADS 4 over a period of 2 years (2009-2011). All had received health services from the same integrated health care system. Data were collected from medical records. Statistical analyses were performed using SAS (version 9.3, Carey NC). Results: Six (1.35\%) men and 437 (98.65\%) women contributed to this study of which, 206 (46.50\%) were African-Americans (AA), 135 (30.47\%) were White-Americans (WA), $31(7.00 \%)$ were classified under other and racial/ethnic information was missing for $71(16.03 \%)$. Of the 393 who underwent biopsy, $346(88.04 \%)$ were diagnosed with inconsequential benign conditions, $25(6.36 \%)$ with proliferative lesions without atypia and $22(5.6 \%)$ with breast cancer. Distribution of pathology diagnostic outcomes among the different racial/ethnic groups approached the level of statistical significance $(\mathrm{P}=.078)$. Clinicopathologic details are listed in tables below.

\begin{tabular}{|c|c|c|c|}
\hline Clinical and radiologic variables & Description & \begin{tabular}{|l} 
Pathologic variables \\
\end{tabular} & Description \\
\hline Age Distribution & & Pathology Outcome & \\
\hline Mean Age & $32( \pm 7)$ years & Benign Conditions & $346(88.04 \%)$ \\
\hline Range & $12-39$ & Proliferative without Atypia & $25(6.36 \%)$ \\
\hline$<20$ years & $38(8.58 \%)$ & Carcinoma & $22(5.60 \%)$ \\
\hline 20-29 years & $86(19.41 \%)$ & Carcinoma & \\
\hline 30-39 & 319 (72.01\%) & DCIS: ER+PR+ & $2(100 \%)$ \\
\hline Reason for Mammography & & Invasive: & $20(100 \%)$ \\
\hline Baseline Screening & & ER+Her2- & $9 / 20(45 \%)$ \\
\hline African Americans & $58(47.5 \%)$ & ER+Her2+ & $5 / 20(25 \%)$ \\
\hline White Americans & $42(34.4 \%$ & ER-PR-Her2- & $4 / 20(20 \%)$ \\
\hline Others & $3(2.46 \%)$ & ER-Her2+ & $2 / 20(10 \%)$ \\
\hline Missing/Declined & $19(15.57 \%)$ & Lymph node+ at diagnosis & $14 / 20(70 \%)$ \\
\hline Total & $122(100 \%)$ & Lymph node-isolated tumor cells & $2 / 20(10 \%)$ \\
\hline Diagnostic & & Distant metastasis at diagnosis & $2 / 20(10 \%)$ \\
\hline African Americans & $148(46.11 \%)$ & African Americans & 9/20(45\%) \\
\hline White Americans & $93(28.97 \%)$ & White Americans & $7 / 20(35 \%)$ \\
\hline Others & $28(8.72 \%)$ & Others & $4 / 20(20 \%)$ \\
\hline Missing /Declined & $52(16.20 \%)$ & Stage grouping: & \\
\hline Total & $321(100 \%)$ & $\mid A, \| A$ & $7 / 20(35 \%)$ \\
\hline Treatment & & IIB & $7 / 20(35 \%)$ \\
\hline No Biopsy & $50(11.29 \%)$ & IIIA, IV & $6 / 20(30 \%)$ \\
\hline Biopsy & $393(88.71 \%)$ & & \\
\hline
\end{tabular}

Conclusions: The incidence of breast cancer under the age of 40 was $5.6 \%$ in this cohort with radiographically detected suspicious lesions (BIRADS-4). Similar to previous reports, aggressive cancer, i.e. diagnosis at a later stage, aggressive phenotype (Her2+, triple negative $\mathrm{BC}$ ) and inferior clinical outcomes were observed. The majority of individuals diagnosed with $\mathrm{BC}$ did not have a family history further emphasizing the necessity of clinical research in discerning the underlying cause of $\mathrm{BC}$ in young adults.

157 Clinical Utility of GATA3 Expression in Cytokeratin-7 Positive Carcinomas

BZ Clark, S Beriwal, DJ Dabbs, R Bhargava. Magee-Womens Hospital of University of Pittsburgh Medical Center, Pittsburgh, PA.

Background: GATA3, a highly-conserved transcription factor in the GATA family, is important in T-cell and mammary gland development, and functions in maintenance of differentiation. GATA3 is a highly sensitive marker in invasive breast carcinoma, with the highest expression in estrogen receptor (ER) positive carcinomas. This study evaluated the utility of GATA3 in differentiating breast carcinoma from other cytokeratin 7 (CK7) positive carcinomas.

Design: GATA3 expression was evaluated by immunohistochemistry in 198 invasive breast carcinomas (181 ductal, 14 lobular, 2 mixed) on tissue microarrays (TMAs). Hormone receptor (HR), HER2 expression, and Nottingham grade were also recorded. TMAs of other tissues included:144 gynecologic (GYN) tumors, 28 bladder carcinomas, 63 cholangiocarcinomas, 20 pancreatic carcinomas, and 63 gastric carcinomas. Full tissue sections of 10 invasive squamous cell carcinomas ( 6 cervical, 4 vulvar) were also stained. GATA3 expression was semiquantitatively scored using an H-score method, ranging from 0 to 300 , which evaluates the percentage of cells staining and intensity of expression. $\mathrm{H}$-score greater than 10 was considered a positive result.

Results: Of 186 evaluable breast carcinomas, 95\% were positive (H-score mean: 217 , median: 230 ): $99 \%$ of HR+/HER2-, $100 \%$ of HER $2+$ tumors, and $73 \%$ of triple-negative carcinomas. GATA3 expression was uncommon in the GYN tumors studied, with $10 \%$ of tumors having $\mathrm{H}$-scores $>10: 17 \%$ of endocervical tumors, $7 \%$ of endometrial tumors, and $10 \%$ of ovarian tumors. Sixty percent of squamous cell carcinomas expressed GATA3 (75\% vulvar, 50\% cervical). Of urothelial carcinomas, 95\% expressed GATA3 (H-score mean: 170, median: 171). Two of 62 cholangiocarcinomas expressed GATA3 $(3 \%)$, with mean and median $\mathrm{H}$-score of 20 . Three of twenty pancreatic adenocarcinomas (15\%) expressed GATA3 (mean and median H-scores 19 and 17 respectively), and only $1 \%$ of gastric carcinomas expressed GATA3, with $1 / 70$ cases positive (H-score 20 ). Conclusions: GATA3 expression is highest in breast and urothelial carcinomas, with high mean and median H-scores. Weaker expression was seen in other carcinomas. GATA3 would not be expected to distinguish urothelial from vulvar/cervical invasive squamous cell carcinoma. In the CK7 positive, ER positive setting, strong GATA3 expression is a reliable marker of breast primary, and can be used in conjunction with other markers to evaluate tissue of origin. GATA3 stains $~ 70 \%$ of triple negative breast carcinomas and could be useful in confirming breast origin in a CK7+, ER negative tumor in the right clinical context.

158 Dual IHC and FISHTesting to Identify HER2 Positive Subpopulations in Breast Cancer: Uncovering Heterogeneity

MR Clay, KH Allison, KC Jensen. Stanford Hospital and Clinics, Stanford, CA.

Background: Identification of distinct clustered subpopulations of HER 2 positive cells in breast cancers can make a difference in patient eligibility for treatment with HER2 targeted therapies. However, appropriate identification and characterization of these subpopulations in heterogeneous cancers can be challenging. Dual testing utilizing the HER2 immunohistochemical (IHC) stain to screen for these subpopulations and inform which areas to focus HER2 fluorescence in situ hybridization (FISH) counting may help appropriately identify these HER2 enriched subpopulations.

Design: We tested 516 sequential breast carcinomas previously reported by HER2 FISH for HER2 status by immunohistochemistry. When the HER2 IHC stain contained areas with different qualitative IHC intensities (IHC heterogeneity) these areas were circled 
and separate FISH counts were performed in each area. The separate FISH scores were then compared to the overall FISH and IHC scores to determine if there would be changes in test interpretation.

Results: Overall, 25 (5\%) cases had IHC heterogeneity by HER2 IHC and were repeat tested and scored by FISH to obtain separate FISH counts in the areas of differential protein expression. In $4 \%$ of the cases with IHC heterogeneity ( 1 of 25 cases), a HER 2 positive subpopulation was identified based on the IHC-targeted FISH testing, in a case that was previously considered HER2 negative. Of cases scored as HER2 2+, targeted FISH scoring yielded a higher FISH ratio in more intensely IHC stained areas in $13 / 19(68 \%)$ cases, while only $2 / 6(33 \%)$ of targeted FISH ratios were increased in cases scored as HER2 $3+$

Conclusions: Although rare (less than $1 \%$ of cases in this series), when variability in HER2 staining by IHC is identified (IHC heterogeneity), targeted FISH counts may identify clinically significant subpopulations. Targeted FISH counts are more likely to increase in more intense areas in cases originally interpreted as HER $22+$ by IHC.

\section{Whole Exome Sequencing (WES): A Pilot Study in Young Women} with Breast Cancer

LC Collins, $N$ Wagle, S Gelber, B Larsen, K Ruddy, S White, E Brachtel, L Schapira, SE Come, VBorges, E Warner, E Winer, A Partridge. Dana-Farber Cancer Center, Boston, MA; Broad Institute of Harvard and MIT, Cambridge, MA; University of Colorado Cancer Center, Aurora, CO; Sunnybrook Odette Cancer Centre, Toronto, Canada.

Background: Advances in next generation sequencing techniques have led to the discovery of genetic mutations in breast cancer enabling identification of potential therapeutic targets beyond standard therapies. Given that young women with breast cancer present with a different distribution of molecular subtypes and clinical outcomes, we sought to investigate whether there were also differences in the distribution of both somatic and germline mutations in this population.

Design: We identified 67 samples from 33 young women with breast cancer: 33 blood and 34 formalin-fixed paraffin embedded (FFPE) tumor samples (1 patient with bilateral cancer). Criteria for inclusion were: age $\leq 36 y$ rs at diagnosis, consent given for genome sequencing, available FFPE tumor blocks and frozen blood samples obtained within 1 year of diagnosis. Core biopsy and neoadjuvant treated specimens were excluded. Genomic DNA was extracted from FFPE tumor blocks utilizing two $1 \mathrm{~mm}$ cores and from matched blood samples. Whole exome sequencing (WES) was performed on tumor and germline DNA at the Broad Institute starting with $<100 \mathrm{ng}$ of DNA. A sample was deemed successfully sequenced if $80 \%$ of the targeted exome achieved depth of coverage of $>20 \mathrm{X}$. Of the successfully sequenced samples, data will be analyzed for point mutations, small insertions/deletions, and copy number alterations using computational algorithms used at the Broad Institute.

Results: WES was successfully completed for 58 of 67 samples $(87 \%)$. Among the $27 / 34$ tumors successfully sequenced, the median percent of the exome covered at $20 \mathrm{X}$ or greater was $89 \%$ (range $79-95 \%$ ). The mean target coverage in this group ranged from $56 \mathrm{X}$ to $222 \mathrm{X}$ (median 99X). Of the $31 / 33$ blood samples successfully sequenced, the median percent of the exome covered at $20 \mathrm{X}$ or greater was $91 \%$ (range $85-94 \%$ ). The mean target coverage ranged from $82 \mathrm{X}$ to $138 \mathrm{X}$ (median 98X). Preliminary analysis of somatic and germline mutational data will be presented.

Conclusions: We present a pilot study of somatic and germline WES from young women with breast cancer. Our results demonstrate that the vast majority of tumor samples - two $1 \mathrm{~mm}$ cores from FFPE tumor tissue - may be used for successful WES. Based on this pilot study, we plan to perform WES in several hundred samples from young women with breast cancer to uncover novel therapeutic targets in these tumors as well as novel germline risk factors for the development of breast cancer at a young age.

160 Subsequent Breast Cancer Risk and Tumor Biomarker Expression According to Mammographic Density: A Nested Case-Control Study

LC Collins, AH Beck, L Yaghjyan, A Pettersson, GA Colditz, B Rosner, S Schnitt, $R$ Tamimi. Beth Israel Deaconess Medical Center, Boston, MA; University of Florida, Gainsville, FL; Channing Institute, Brigham and Women's Hospital, Boston, MA; Washington University, St. Louis, MO.

Background: Studies evaluating risk factors and breast cancer molecular subtype indicate a relationship between body weight metrics and history of breast feeding with the development of basal-like breast cancer. Few have looked at mammographic density and subsequent molecular subtype or tumor biomarker expression.

Design: We conducted a case-control study of mammographic density and breast cancer risk nested within the Nurses' Health Study. The analysis included 1,154 postmenopausal women diagnosed with breast cancer between 1989-2004, and 2,078 matched controls without breast cancer. Percent breast density, absolute dense and nondense areas were estimated from digitized mammograms using computer-assistance and categorized into quartiles. Estrogen and progesterone receptor (ER, PR), HER2, cytokeratin $5 / 6$, epidermal growth factor receptor (EGFR) and androgen receptor (AR) were evaluated on immunostained tissue microarrays of the cancers or from pathology reports. Polychotomous logistic regression was used to assess associations of breast density measures with tumor biomarkers.

Results: Percent density and absolute dense area were positively associated with breast cancer risk whereas an inverse association was seen for absolute non-dense area ( $p$ for trend $<0.0001$ for all). We found strong positive associations of absolute dense area with CK5/6- and EGFR- tumors compared to biomarker-positive tumors (4th vs. 1st quartile: $\mathrm{CK} 5 / 6-\mathrm{OR}=3.0$ vs. $\mathrm{CK} 5 / 6+\mathrm{OR}=0.9$, p-heterogeneity $<0.001$; EGFR-OR=3.1 vs. $\mathrm{EGFR}+\mathrm{OR}=1.1, \mathrm{p}$-heterogeneity $=0.001)$. There was an inverse association of absolute non-dense area with breast tumors that were ER-, AR+, CK5/6+, and EGFR+ (4th vs. 1 st quartile: $\mathrm{ER}-\mathrm{OR}=0.2$ vs. $\mathrm{ER}+\mathrm{OR}=0.6$, p-heterogeneity $<0.0001 ; \mathrm{AR}+\mathrm{OR}=0.2$ vs. $\mathrm{AR}-\mathrm{OR}=0.4$, p-heterogeneity $=0.04 ; \mathrm{CK} 5 / 6+\mathrm{OR}=0.2$ vs. $\mathrm{CK} 5 / 6-\mathrm{OR}=1.1$, p-heterogeneity $<0.01 ; \mathrm{EGFR}+\mathrm{OR}=0.4$ vs. $\mathrm{EGFR}-\mathrm{OR}=1.1$, p-heterogeneity $<0.01)$. The associations of percent density with breast cancer risk did not differ significantly by biomarker status, nor were there any differences in associations of any of the density measures with PR or HER2.

Conclusions: Our findings suggest that some mammographic density measures are differentially associated with the risk of subsequent breast tumor subtypes; in particular absolute non-dense area appears inversely associated with more aggressive tumor subtypes such as ER negative, basal cytokeratin and EGFR positive tumors.

\section{Is the Rate of Metastatic Breast Cancer Increasing in Young Women?}

$N$ Conlon, J Howard, J Catalano, M Gallagher, LK Tan, C Adriana. MSKCC, New York, NY.

Background: A recent report based on SEER data identified a significant increase in the incidence of breast cancer with metastatic (M1) disease at diagnosis among young patients (aged less than 40) over the last few decades, particularly in patients with estrogen receptor positive (ER + ) disease. Further, there is a widespread anecdotal belief among pathologists and clinicians that greater numbers of young women are presenting with breast cancer in recent years. We undertook to study whether these hypotheses were true for the patient population treated at MSKCC from 2000 to 2013. Design: Clinicopathologic data on patient age, diagnosis, clinical and pathologic stage, hormone receptor status and HER2 overexpression status was compiled for the period 2000-2013, including all patients who received treatment at MSKCC (all patients $\mathrm{n}=$ 24,830 ; patients $<40$ years $n=2,488$ ). Sub-group analysis was performed based on the above parameters.

Results: Over the study period, the proportion of breast carcinoma cases occurring in young patients $(<40$ years of age) declined slightly $(11.9 \%$ to $9.6 \%$; $<0.001)$. Young patients were more likely than older patients to present with metastatic disease $(6.06 \%$ vs $4.9 \% ; \mathrm{p} 0.04$ ) but this rate remained steady over the study period and no differences were noted based on ER status. Evidence of stage migration in M0 disease was identified, with an increasing proportion of young patients presenting with localized disease over the study period $(41.67 \%$ vs $51.2 \% ; \mathrm{p}=0.0003)$. Breast cancer in young patients was more likely to be triple negative $(20.5 \%$ vs $13.6 \%$; $<0.001)$ and HER $2+(p<0.01)$. Young patients with HER2+ cancers were significantly more likely to present with M1 disease $(9.6 \%$ vs $4.8 \% ; \mathrm{p}=0.0002)$; in patients with M0 tumors, young patients with HER $2+$ tumors were significantly more likely to have regional disease $(56 \%$ vs $47.6 \% ; \mathrm{p}=0.04)$.

Conclusions: Our study shows that there has been no demonstrable increase in the relative proportion of breast cancer in patients $<40$ years at MSKCC between 2000 and 2013, and contrary to a recent SEER report, no increase in the proportion of young patients presenting with M1 disease. However, young patients with HER2+ tumors were significantly more likely to present with M1 disease, and to have regional metastases at presentation.

162 Benign and Low-Grade Fibroepithelial Neoplasms of the Breast Have Low Recurrence Rate after Positive Surgical Margins

M Cowan, P Argani, A Cimino-Mathews. The Johns Hopkins Hospital, Baltimore, MD. Background: Breast phyllodes tumors are fibroepithelial lesions with variable risk of local recurrence and metastasis. The benign and low-grade fibroepithelial neoplasms are on a morphologic spectrum that overlaps between from fibroadenomas with phyllodal features (FPF), benign phyllodes (BP) and borderline phyllodes (BLP). Positive surgical margin status has been correlated with local recurrences, particularly in the high-grade malignant phyllodes (MP). However, the surgical management of positive margins in patients with low-grade fibroepithelial neoplasms is variable, and not all patients undergo margin re-excision. Here, we review the margin status, re-excision rate, and recurrence rate in a series of patients with low-grade fibroepithelial neoplasms of the breast.

Design: Pathology archives were searched for patients with benign phyllodes and borderline phyllodes tumors excised at our institution from 1989-2013, with available clinicopathologic data and follow-up. In addition, a consecutive series of patients diagnosed with fibroadenomas with phyllodal features (lesions with overlapping features of fibroadenomas and benign phyllodes) were included for comparison. In total, 89 patient's tumors were studied: 19 FPF, 55 BP, and 15 BLP. Surgical pathology reports and clinical records were reviewed.

Results: In the 89 patients with low-grade fibroepithelial lesions, $75 \%$ had positive surgical margins at first resection (95\% FAP, 73\% BP, 73\% BLP) and only 33\% had subsequent margin re-excision ( $11 \%$ FAP, $40 \%$ BP, $45 \%$ BLP) while $66 \%$ had no additional surgery ( $89 \% \mathrm{FAP}, 60 \% \mathrm{BP}, 55 \% \mathrm{BLP})$. Of the 23 patients with re-excision of positive margins, only $4(17 \%)$ had residual tumor in the re-excised specimen, all with negative final margins. Of the 46 cases with no re-excision for positive margins, only 1 patient $(2 \%)$ developed a recurrent tumor, which was a FAP that recurred as a BP. Two of the $23(9 \%)$ patients who underwent re-excision to clear positive margins also developed recurrent tumors; one BP recurred as BP, and one BLP recurred as a MP. This latter patient developed metastatic MP and died due to disease.

Conclusions: These findings suggest that patients with low-grade fibroepithelial lesions of the breast have low risk of local recurrence despite positive surgical margins. These patients may be adequately managed by close clinical follow-up with timely re-excision of any subsequent lesion. Patients who undergo re-excision to obtain negative margins also need close clinical follow-up, as they may also develop recurrences. 
163 Discordance between HER2 Copies/Cell and HER2/CEP17 Ratio by Fluorescence In Situ Hybridization (FISH) in Invasive Breast Carcinoma A Czapla, JF Silverman, U Krishnamurti. Allegheny General Hospital, Pittsburgh, PA. Background: According to the 2006 ASCO/CAP guidelines for HER2 testing in breast cancer: Positive for HER2 is either IHC $3+$ or FISH amplified (HER2/CEP17 ratio > 2.2 or mean $H E R 2$ copy number $>6$ signals/cell for systems with a single $H E R 2$ probe) and negative for HER 2 is either IHC $0-1+$ or $<1.8$ HER $2 / C E P 17$ ratio by FISH or mean $H E R 2$ copy number $<4$ signals/cell for systems with a single $H E R 2$ probe. A small percentage of cases of invasive breast carcinoma (IBC) have increased centromere 17 copy numbers with or without HER2 amplification. The aim of this study was to evaluate the discordance in reporting HER2 amplification by FISH based on average $H E R 2$ copies/cell and $H E R 2 / C E P 17$ ratio.

Design: In IBC cases that underwent FISH for HER between 2005-12, the absolute number of $H E R 2$ copies/cell was examined in HER 2 amplified and in unamplified cases with increased chromosome 17 copy numbers.

Results: Of 1850 cases of IBC that had FISH for HER2, 279 cases were reported as having increased CEP17 numbers without HER 2 amplification. Of these 279 cases, 101 cases $(36.2 \%)$ had $<4$ HER 2 copies/cell and 30 cases $(10.8 \%)$ had $>6$ HER2 copies/cell. 29 of these cases with $>6$ HER2 copies/cell had 6-9 HER2 copies/cell with a mean of 7.0 HER2 copies/cell. From 2005-2012, 396 cases were reported as HER2 amplified. Of these, 221 cases had only IHC performed (were 3+ positive by IHC) and 175 cases had both IHC and FISH performed. There was $95 \%$ concordance between cases $3+$ positive by IHC and FISH. From 2007 onwards FISH was mainly performed if IHC was 2+. Overall of the 396 HER2 amplified cases, 5 cases $(1.3 \%)$ had $<4$ HER2 copies/cell and 33 cases $(8.3 \%)$ had 4-6 HER2 copies/cell. Thus $9.6 \%$ of HER2 amplified cases with a HER2/CEP17 ratio $>2.2$ had $<6 H E R 2$ copies/cell. These would not have been interpreted as HER2 amplified in test systems without an internal CEP17 control probe. On the other hand, $10.8 \%$ of cases reported as negative for HER2 amplification with increased chromosome 17 copy numbers based on a HER2/ CEP 17 ratio $<1.8$ had $>6$ HER 2 copies/cell. These would have been interpreted as HER 2 amplified in test systems without an internal control probe.

Conclusions: About $10 \%$ cases show discordance between HER2 copies/cell and HER 2/CEP17 ratio by FISH. The use of CEP 17 to correct HER 2 copy numbers may give misleading results. In cases tested by dual color FISH, we believe that clinical decision regarding therapy should be made and trials testing efficacy of anti-HER2 agents should not only select patients on the basis of $H E R 2 / C E P 17$ ratio, but also on absolute $H E R 2$ copy numbers.

164 Study of an Open Breast Cancer Dataset:The Value of Gene-Based Assays Is in Further Stratifying Intermediate Grade Cancers

\section{LW Dalton. South Austin Medical Center, Austin, TX.}

Background: An open dataset (specifically the NKI dataset) of 295 patients with breast cancer provided results from several gene-based assays [GBAs] to include recurrence score [RS], and the seventy gene assay [70G] (Fan et al. N Engl J Med 2006;355:560-9) An open dataset allowed for possible validation of a simple rule that incorporated GBAs together with low, intermediate and high histologic grade [LG,IG,HG].

Design: A very simple decision tree rule [DTR] was proposed. LG and HG tumors were considered terminal nodes. IG cancers were subdivided by GBAs into IG-low risk and IG-high risk subsets. Subsequently, LG was combined with IG-low risk and HG combined with IG-high risk. The NEJM data was used to validate the rule (or not). Results: As an example with 70G: LG versus IG-70Ggood showed no statistical significance in survival, nor did HG versus IG-70Gpoor. When LG was combined with IG-70Ggood, and likewise HG with IG-70Gpoor, there was significance achieved in a low risk versus high risk categorization $(\mathrm{p}<0.00001)$. The $70 \mathrm{G}$ assay was able to significantly further stratify IG $(\mathrm{p}<0.001)$, but not LG or HG (respectively $\mathrm{p}=0.1$ and $\mathrm{p}=0.25$ ). The NEJM paper had considered a Cramer's V [CrV] statistic of over 0.5 as indicating strong concordance between two attributes. The DTR-70G had $0.57 \mathrm{CrV}$ with RShigh, and CrV of $70 \mathrm{G}$ with RShigh was 0.58 . Despite two-thirds substitution of $70 \mathrm{G}$ by $\mathrm{LG} / \mathrm{HG}$ there was no loss in concordance.

Conclusions: Discovered from the very dataset used to validate the $70 \mathrm{G}$ assay (the NKI dataset), it is reasonable to conclude that further testing by GBAs (having the same goal of grade), be limited to IG cancers. A common theme of GBAs, and immunohistochemical surrogates, is in measuring tumor proliferation. Further studies of $\mathrm{HG}$ and LG, by any proliferation biomarker, may provide nothing but troublesome discrepancies. Value is achieved in use of proliferation biomarkers to further stratify IG. This is corroborated by similar studies, on other datasets, to include a Nottingham study of Ki-67. Yet, finding this DTR to be true in the NKI dataset is of special interest because of the role the NKI dataset has played in the validation of GBAs.

165 Comparison of PELP1 and GATA3 Expression in Triple-Negative Breast Carcinoma: Potential Diagnostic Utility in Metastatic Disease DN Dang, G Raj, V Sarode, RK Vadlamudi, Y Peng. UT Southwestern, Dallas, TX; UTHSCSA, San Antonio, TX.

Background: PELP1 is a novel coregulator of the estrogen receptor(ER), and GATA3's expression is highly correlated with ER in breast cancer. Recent studies revealed both markers play a critical role in ER-positive breast cancers. However, their roles have not been well characterized in triple-negative breast carcinoma (TNBC). Diagnosing metastatic TNBC is challenging due to the TN immunophenotype and limited sensitivity of currently used markers. A recent molecular study suggested PELP1 may be a therapeutic target for metastatic ER-negative breast cancer. Our study aims to examine these markers' expression in primary and metastatic TNBCs in order to explore a new ancillary tool in the diagnosis of metastatic TNBC.
Design: $72 \mathrm{TN}$ invasive carcinoma cases were identified at our institution. 15 of the $72(21 \%)$ cases were metastatic TNBCs. Tissue microarrays (TMAs) were constructed with duplicate cores of each tumor and of adjacent normal breast ducts. The TMAs immunolabeled for PELP1 and GATA3 were scored based on extent of nuclear staining as follows: 0 (negative), $1(1-30 \%), 2(31-60 \%), 3(61-90 \%)$ or $4(91-100 \%)$. The intensity of the staining was graded as weak, moderate or strong. The slides were reviewed and scored by two pathologists, and an average score for each case was used for statistical analysis.

Results: 70 of $72(97 \%)$ TN tumors were found to have nuclear PELP1 expression (mean score $3.81 \pm 0.09 ; 49$ strong, 15 moderate, 7 weak). PELP1 stained $92 \%$ of paired normal ducts (mean score $3.56 \pm 0.17)$. Only 9 tumors of $72(12.5 \%)$ showed nuclear GATA3 expression (mean score $0.32 \pm 0.11 ; 3$ strong, 5 moderate, 1 weak). GATA3 stained $84 \%$ of paired normal ducts (mean score $1.86 \pm 0.16$; most with moderate or strong intensity). 14 of 15 (93\%) metastatic tumors showed diffuse and strong PELP1 staining while only one metastatic tumor $(6.7 \%)$ showed focal (score 1), weak GATA3 staining. PELP1 has a significantly higher mean expression level and frequency of expression in TNBCs than GATA3 ( $\mathrm{p}<0.0001)$.

Conclusions: Our results indicate PELP1 appears to be an effective diagnostic biomarker for TNBC with $97 \%$ sensitivity, significantly more sensitive than GATA3. Although its specificity needs to be further defined, PELP1 has potential diagnostic utility for metastatic TNBC and can be included in IHC panels for work-up of tumors of unknown origin with suspicion for primary TNBC. In this study, PELP1 is diffusely and strongly expressed in $93 \%$ of human metastatic TNBCs, supporting the proposal that PELP1 may be a therapeutic target for metastatic TNBC.

166 Lymph Vessel Density as Determined by Computerized Image Analysis of D2-40 Expression Does Not Correlate with Clinicopathological Features and Outcome in Triple-Negative Breast Cancer

M De Brot, RM Rocha, FA Soares, H Gobbi. Universidade Federal de Minas Gerais, Belo Horizonte, Minas Gerais, Brazil; A.C. Camargo Cancer Center, São Paulo, Brazil. Background: Triple-negative breast cancers (TNBC) tend to relapse and metastasize early with a distinct pattern of dissemination. Although axillary node status represents one of the main parameters in determining prognosis in breast cancer, lymph vessel research has evolved slowly compared with that in blood vessels. Prognostic significance of lymphangiogenesis and its surrogate marker, lymph vessel density (LVD), in TNBC remains controversial. We assessed lymph vessel topography and LVD in TNBC and examined their association with clinicopathological criteria, basal-like phenotype and outcome.

Design: Whole tissue sections from 133 TNBC were stained with D2-40; slides were scanned using Aperio Digital Pathology System. Each digital slide was examined by a pathologist and divided into regions: intratumoral (IT), peripheral (PP), peritumoral (PT). LVD was measured by the Microvessel Density $v 1$ algorithm. Instead of counting vessels in hot spots, we adopted a modified method by assessing all lymph vessels in each area (area $=34.0 \mathrm{~mm}^{2}$ each). LVD was categorized into high and low according to its median value.

Results: Median follow-up time was 40 months (range, 1-161 mos) and 53 (39.1\%) pts had developed a recurrence by the time of last follow-up; distant metastasis was detected in $49(36.8 \%)$ pts, lungs, brain and bones representing the main sites. Overall, $101(75.9 \%)$ TNBC showed a basal phenotype. Lymphatics were located largely in the PP area. PT lymphatics were detected in $98(73.7 \%)$ tumors while PP lymphatics were observed in 116 tumors $(87.2 \%)$; $92(69.2 \%)$ tumors had IT lymphatics. In the normal breast, lymphatics were often noted in the interlobular stroma, but completely absent from the intralobular compartment. LVD in the PT area ranged from 0.0 to $8.1 / \mathrm{mm}^{2}$ (median $0.25 / \mathrm{mm}^{2}$ ), from 0.0 to $27.4 / \mathrm{mm}^{2}$ (median $0.6 / \mathrm{mm}^{2}$ ) in the PP area, and 0.0 to $6.8 / \mathrm{mm}^{2}$ (median $0.1 / \mathrm{mm}^{2}$ ) in the IT area. No significant associations were found between LVD and age, stage, tumor size, node status, histologic type/grade, basal phenotype. There was a trend for association of high total LVD with distant metastasis $(\mathrm{p}=0.07)$. Survival analysis revealed no association between LVD, overall and diseasefree survival. Conversely, survival was associated with stage $(\mathrm{p}=0.04)$, node status $(\mathrm{p}=0.03)$ and distant metastasis $(\mathrm{p}=0.00)$.

Conclusions: Our data show no prognostic impact of LVD in TNBC; standard pathologic parameters determine outcome.

167 Assosication with Clinicopathological Parameters and Survival

L Demir, S Yigit, A Sari, D Etit, A Avci, MO Tarhan, F Cakalagaoglu. Katip Celebi University Ataturk Training and Research Hospital, Izmir, Turkey.

Background: Macrophages constitute a major component of the leucocytic infiltrate of tumours. In the present study we investigated in association prognosis and clinicopathological parameters with CD68 as a macrophage marker at local advanced breast carcinomas.

Design: We searched the pathology archives between 2005 and 2012, identifying 60 local advanced breast cancer cases who had neoadjuvant treatment. Immunohistochemical analysis were performed on paraffin-embedded blocks with monoclonal anti-CD68 antibody [KP1] (abcam clone ab 955, 1/200 dilution). Cytoplasmic staining was used as positive staining in macrophages. The median number of CD 68 positive cells for study group was determined, values below the median level were defined as low expression of CD68 and values above the median value were defined as high expression.

Results: The median age at the diagnosis was $49.85 \%$ of tumors were invasive ductal, $6.7 \%$ were invasive lobular $3.4 \%$ were mixed breast carcinoma, $1.7 \%$ invasive micropapillary carcinoma and $1.7 \%$ invasive papillary carcinoma. Nuclear grade (NG) $71,7 \%$ of tumors grade $2,23,3 \%$ were grade 3 and $5 \%$ were grade 1 . While hormone receptor status was positive in majority of tumors, $60 \%$. $68,3 \%$ of the tumours were Her-2 negative.The median number of CD68 was $18,1 \pm$ SD 7,29 (min 3,8-max 41,8) 
with high expression in $75 \%$ of the NG 3 (high NG) patients and in $41.3 \%$ of NG1 and 2 (low NG) tumors $(\mathbf{p}=\mathbf{0 . 0 0 4})$. Therefore, $73.7 \%$ of HER-2 (+) patients had high expression of CD68, the ratio was noted $41.4 \%$ in HER 2 (-) patients $(\mathbf{p}=\mathbf{0 . 0 2})$. The median follow-up time of the patients was 27.7 months $(5.7-90.9$ months) and the mean of estimated survival time was 47.4 months ( $\min 40.7$-max 54.4 months). Mean expected life time of the patients with high expression CD 68 was 40.7 months while 54.2 months in low expression CD68 patients.

Conclusions: Accordig to the current study, higher CD68 expression implies a lesser estimated life time for the patient in locally advanced breast cancer and high rate of $\mathrm{HER}(+)$ and high NG can be meaningful for a worse outcome. Further study is required to determine clinical importance on this issue.

168 Deletion of EGFR Exon 19 in Invasive Lobular Breast Carcinoma M DeNicola, RT Phan, P Nham, NA Moatamed. David Geffen School of Medicine at UCLA, Los Angeles, CA; VA Greater Los Angeles Healthcare System, Los Angeles, CA. Background: Epidermal growth factor receptor (EGFR) is a tyrosine kinase receptor mediating cell proliferation through the RAS-RAF pathway. EGFR mutations have been reported in triple negative breast cancers. This study examines EGFR, KRAS and BRAF gene mutations in breast cancers irrespective of reactivity for hormone receptors and Her2/neu status. Hormone receptor positive breast cancers often benefit from endocrine therapy. However, resistance to treatment can develop. The resistance appears to be a result of down-regulation of growth factor and protein kinase signaling pathway.

Design: 87 patients, ages 32-89 years, were randomly selected among the breast carcinomas diagnosed between 2008-2010. 67 cases were invasive ductal carcinoma (IDCa) and 13 were invasive lobular carcinoma (ILCa). The remaining 7 cases were invasive metaplastic carcinoma (IMCa) and invasive tubular carcinoma (ITCa). Table 1 demonstrates the subtype of carcinoma, KRAS, BRAF, EGFR, hormone receptor status, Her2/neu status by FISH, and lymph node involvement. DNA was extracted from paraffin blocks and real-time polymerase chain reaction was performed to screen for mutations of EGFR, KRAS, and BRAF. DNA sequencing was also performed to validate and confirm the results.

Results: Only two of the 87 cases exhibited EGFR mutation. Both cases were ILCa (2 of $13,15 \%$ ), and both showed lymph node involvement and were ER positive. EGFR mutation in both cases was deletion of exon 19. These findings are summarized in Table 2. None of the cases showed KRAS or BRAF mutations.

Table 1. Summary of different breast carcinomas with KRAS/BRAF, EGFR, ER, PR, Her2/neu,

\begin{tabular}{|c|c|c|c|c|c|c|c|}
\hline & $\begin{array}{l}\text { Number of } \\
\text { Patients }\end{array}$ & KRAS/BRAF + & EGFR + & ER+ + & $\mathrm{PR}+$ & HER $2 /$ neu by FISH + & \begin{tabular}{|l} 
Lymph \\
Nodes \\
+
\end{tabular} \\
\hline $\mathrm{DCa}$ & 67 & $0 / 0$ & 0 & 57 & 47 & 10 & 25 \\
\hline IMCa & 3 & $0 / 0$ & 0 & 0 & 0 & 0 & 1 \\
\hline ITCa & 4 & $0 / 0$ & 0 & 4 & 4 & 0 & $\sqrt{1}$ \\
\hline ILCa & 13 & $0 / 0$ & 2 & 13 & 8 & 0 & $\sqrt{3}$ \\
\hline
\end{tabular}

Table 2. Results of invasive lobular carcinoma with EGFR, ER, PR, Her2/neu, and lymph node status

\begin{tabular}{||l|l||l||l|l|l||}
\hline \hline & Number of Patients & ER + & PR+ & HER2/neu by FISH + & Lymph Nodes + \\
\hline EGFR + & 2 & 2 & 0 & 0 & 0 \\
\hline EGFR- & 11 & 11 & 8 & 0 & 2 \\
\hline \hline
\end{tabular}

Conclusions: This study is among the first to document EGFR mutation in ER+, lymph node positive ILCa cases. In addition, this study demonstrates that KRAS and BRAF genes are not mutated in breast cancer. These findings, once substantiated in larger series of ILCa, provide significant implication for potential usage of EGFR-targeted therapy in breast cancer treatment.

169 Decreased Mitochondrial SIRT3 Expression Is Potential Molecular Biomarker Associated with Poor Outcome in Breast Cancer

MM Desouki, I Doubinskaia, D Gius, SA Abdulkadir. Vanderbilt University, Nashville, TN; Northwestern University, Chicago, IL.

Background: SIRT3 is a genomically expressed, mitochondrial localized tumor suppressor protein (TSP) where it directs multiple metabolic processes by deacetylating downstream protein substrates. Genetic deletion of Sirt3 in mice leads to spontaneous development of mammary tumors and decreased SIRT3 mRNA has been observed in several human tumors including breast malignancies.

Design: We assessed SIRT3 expression in human breast cancer tissue microarrays by immunohistochemistry (IHC) and examined the relationship between SIRT3 expression and outcome in breast cancer patients.

Results: SIRT3 expression by IHC is significantly lower in breast cancer compared to normal breast epithelium, including normal epithelium adjacent to tumors. $23 \%, 72 \%$ and $74 \%$ of normal breast tissue, in situ lesions and invasive carcinomas were negative for SIRT3 expression, respectively $(\mathrm{p}<0.001)$. No statistically significant difference in SIRT3 expression across tumor grades. SIRT3 loss occurs with a higher frequency in ER- ( $83 \%$ vs $62 \%)$ and PR- ( 82 vs $68 \%$ ) compared to ER+ and PR+ tumors. SIRT3 expression in relation to the HER2 status showed no significant difference. $75 \%$ of triple negative tumors showed loss of SIRT3 expression. Breast cancer patients in the lowest quartile of SIRT3 expression had a significantly shorter relapse free survival [HR= $0.53(0.47-0.61), \log$ rank $\mathrm{p}=0]$. Notably, low SIRT3 expression was associated with reduced relapse-free survival in all breast cancer subtypes analyzed: ER+, ER-, HER2+, luminal $\mathrm{A}$, luminal $\mathrm{B}$ and basal subtypes ( $\mathrm{HRs}=0.44$ to 0.65 ; $\log$ ranks $\mathrm{p}=0$ to 0.0019 ).
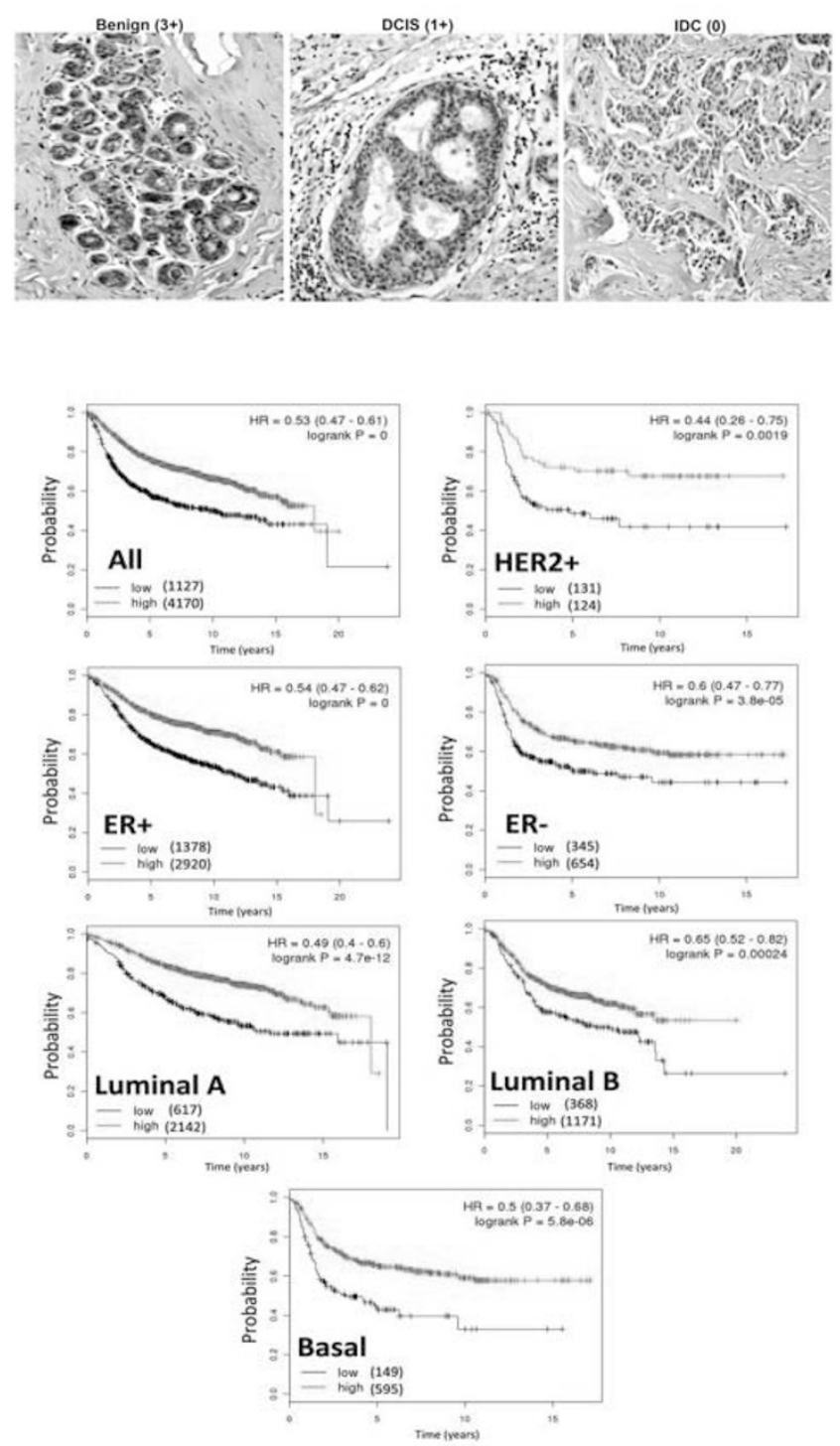

Conclusions: These results highlight the importance of the SIRT3 as a tumor suppresor protein in breast cancer and suggest that SIRT3 may be a potential molecular biomarker to identify high risk patients across all molecular subtypes of breast cancer.

170 The Rate of Upgrade for Lobular Neoplasia in MRI-Guided Core Needle Biopsy: A Study of $\mathbf{6 3}$ Cases from Four Different Institutions

$M M$ Desouki, R Karabakhtsian, S Sanati, Z Li, B Reig, P Kumar, X Chen, D Wang, X Huang, S Liu, T Khoury. Vanderbilt University, Nashville, TN; Montefiore Medical Center, New York, NY; Washington University, St Louis, MI; University of Pittsburgh Medical Center, Pittsburgh, PA; Roswell Park Cancer Institute, Buffalo, NY.

Background: There are certain criteria to recommend surgical excision for lobular neoplasia (LN) diagnosed on mammographically detected core needle biopsy (CNB). These criteria include patients whom the pathologic finding of $\mathrm{LN}$ does not account for the mammographic abnormality; or the LN is accompanied by another lesion which by itself would warrant excision. With the increase use of the MRI in breast imaging, the rate of $\mathrm{LN}$ in this setting has increased, which dictates the need for similar criteria on MRI-detected LN

Design: We reviewed 1594 MRI-guided CNB from four institutions over 7-yers to yield $63(3.95 \%)$ cases. LN was classified into atypical lobular hyperplasia (ALN), classic lobular carcinoma in situ (LCIS) and pleomorphic LCIS (PLCIS). Concurrent atypia was also recorded including atypical ductal hyperplasia (ADH), flat epithelial atypia (FEA), papillomas and radial scars (RS). MRI findings including mass-enhancement (ME) vs. non-mass-enhancement (NME), and the reason for biopsy (high risk screening, staging, equivocal mammogram, and clinical suspicion) were also recorded. An upgrade was considered when the surgical excision yielded invasive carcinoma or ductal carcinoma in situ. Fisher exact test was used for statistical analysis.

Results: The upgrade rate identified was $13(20.63 \%)$ of all cases (mixed and pure). There were $34(53.97 \%)$ cases with pure LN. There was no statistically significant difference in the rate of upgrade between pure LN ( 9 of $34,26.47 \%$ ) and mixed LN (4 of $29,13.79 \%$ ). Concurrent FEA ADH, papilloma and RS were found in $8(12.7 \%)$, $16(25.4 \%), 4(6.3 \%)$, and $9(14.3 \%)$ cases, respectively. Table 1 illustrates the rate of upgrade for each variable. 
Conclusions: While the incidence of LN in MRI-guided CNB is relatively low, the risk of upgrade is relatively high. Unlike in mammographically detected LN, concurrent atypia, such as $\mathrm{ADH}$, does not increase the risk of upgrade.

\begin{tabular}{|c|c|c|}
\hline Variable(total) & Upgrade-No & Upgrade-Yes \\
\hline \multicolumn{3}{|l|}{ Histologic type } \\
\hline ALH(29) & $24(48)^{*}$ & $5(38)$ \\
\hline $\operatorname{LCIS}(31)$ & $24(48)$ & $7(54)$ \\
\hline $\operatorname{PLCIS}(3)$ & $2(4)$ & $1(8)$ \\
\hline \multicolumn{3}{|l|}{ MRI findings } \\
\hline Mass(24) & $22(44)$ & $2(15)$ \\
\hline Non-mass(39) & $28(56)$ & $11(85)$ \\
\hline \multicolumn{3}{|l|}{ Reason for MRI } \\
\hline Equivocal mammogram(4) & $3(6)$ & $1(8)$ \\
\hline Clinical suspicion(1) & $1(2)$ & $0(0)$ \\
\hline High risk(28) & $20(41)$ & $8(62)$ \\
\hline Staging (29) & $25(51)$ & $4(31)$ \\
\hline
\end{tabular}

*N(\%); none of the comparisons was statistically significant.

\section{$171 \quad$ High Nuclear Expression of p27 Correlates with Luminal A} Molecular Subtype of Ductal Carcinoma In Situ of Breast

S Dhamne, N Sneige, D Rosen, I Bedrosian, E Resetkova, Y Wu, B Arun, C Albarracin. Baylor College of Medicine, Houston, TX; The University of Texas MD Anderson Cancer Center, Houston, TX; Micheal E. DeBakey VA Medical Center, Houston, TX. Background: Ductal carcinoma in situ (DCIS) is a heterogeneous disease with differences in pathologic features, clinical behavior and molecular characteristics. Though traditionally, grade of DCIS is considered an important prognostic marker, other key significant prognostic and predictive markers associated with invasive breast cancer have not been well studied in DCIS. Hence there is a need to identify molecular biomarkers that can help predict behavior and differentiate indolent from aggressive DCIS to better tailor therapy. The objective of this study was as follows: 1) evaluate expression of cell cycle proteins with clinicopathologic features of DCIS, 2) analyze the grade-dependent expression of cell cycle proteins and correlate with routine biomarker expression.

Design: In this retrospective study from 2000 - 2011, diagnostic slides and clinical information were available for 65 cases of DCIS presenting as the primary lesion. Grade was evaluated using criteria from Lagios et al by two breast pathologists and an aspiring breast fellow. In case of discrepancy, final grade was decided by consensus. Paraffin locks with sufficient material for tissue microarrays were available for 47 cases (6 low grade, 20 intermediate and 21 high grade). A detailed histopathologic evaluation including laterality, size, nuclear grade, growth pattern, comedonecrosis, associated microcalcifications, expression of biologic markers ER, PR, HER2, Ki-67 and the proliferation pathway $\mathrm{p} 21, \mathrm{p} 27$, cyclin D1.

Results: Nuclear expression of p27 was seen in majority ( 44 cases, $93.6 \%$ ) of DCIS with a mean nuclear expression of $64.5 \%(20-95 \%)$. DCIS with high nuclear expression of p27 were statistically significant correlated with ER+, PR+ and HER2 negative status. Intensity of the cytoplasmic expression of p27 when present did not correlate with grade of DCIS; however cytoplasmic p 27 was distinctly absent in the surrounding normal breast tissue wherever present ( 39 cases). p2 7 expression did not correlate with nuclear grade, age, size, growth pattern and presence or absence of comedonecrosis, p21, Ki-67 and cyclin D1. No other cell marker showed any significant correlation. Conclusions: High nuclear expression of the cell cycle inhibitor, p27 in DCIS correlated with $\mathrm{ER}+, \mathrm{PR}+$ and HER2 negative status.

172 Diagnostic Yield of Magnetic Resonance Imaging (MRI)-Guided Core Needle Biopsies for Early Detection of Breast Cancer in a Cohort of 546 Patients

S Dhingra, N Sneige, MR Schwartz, JA Roberts, AG Ayala, JY Ro. Houston Methodist Hospital, Houston, TX.

Background: MRI is increasingly being used as an adjunctive diagnostic modality for early detection of breast cancer. It is also utilized to guide surgical management and staging in patients with a new diagnosis of breast cancer. However, there is very limited data in the literature regarding the yield of high-risk lesions in core needle biopsies of breast (CNBB) obtained using this modality. The aim of this study was to evaluate the clinical value of MRI in breast by assessing the diagnostic yield of malignant lesions, as well as other high to intermediate risk lesions that require active therapeutic intervention, in MRI-guided CNBB.

Design: The study involved a retrospective review of biopsy reports of MRI-guided CNBB, received in our department over a period of 6 years (2006-2012).

Results: A total of 689 MRI-guided CNBB were obtained from 546 patients (pts) with a mean age of 55 years (range 23-85 years). Breast enhancement on MRI was the main indication to obtain biopsies in patients with history of high risk or cancer on initial screening. A quarter of the patients $(n=134)$ had biopsies from 2 or more sites and the majority $(98 \%)$ were unilateral. Invasive ductal carcinoma (IDC) was detected in $32(4.7 \%)$ biopsies (IDC in 24 and invasive lobular carcinoma (ILC) in 8). Ductal carcinoma in situ (DCIS) was diagnosed in an additional 33 (4.8\%) biopsies. NonHodgkin lymphoma was detected in two cases. High-risk lesions such as atypical ductal hyperplasia and lobular neoplasia were detected in $72(10.4 \%)$ biopsies. Intermediate risk lesions, such as radial scar, flat epithelial atypia, papilloma and papillomatosis, that may require active therapeutic intervention, were detected in $111(16.1 \%)$ biopsies. Benign lesions were seen in $439(63.7 \%)$ biopsies.

Conclusions: In this study, the overall rate of malignancy detected by MRI-guided biopsies was $9.8 \%$ (IDC carcinoma, $4.7 \%$, DCIS, $4.8 \%$ and other, $0.3 \%$ ) and the rate of high risk proliferative lesions was $10.4 \%$, for a combined total of $20.2 \%$. Although most biopsied MRI abnormalities are benign, biopsy of MRI abnormalities is warranted given the significant detection of malignancy and high risk lesions.

173 Effect of Prolonged Fixation Time on ER, PR and Her2 Immunohistochemistry in Breast Cancer

$M$ Dilly, M Karanian-Philippe, S Croce, R Jacquemart, MC Petersen, G MacGrogan. Institut Bergonie, Bordeaux, France.

Background: ASCO and CAP recommend fixing breast tumor samples in $10 \%$ neutral buffered formalin (NBF) no longer than 48 hours for optimal Her2 immunohistochemistry (IHC) and 72 hours for Estrogen Receptor (ER) and Progesterone Receptor (PR) IHC. These periods are frequently overrun in our laboratory, especially for surgical samples that are sent at end of the week.

Design: 32 consecutive breast cancer surgical specimens with tumors measuring more than $20 \mathrm{~mm}$, collected over a period of 11 weeks, were included in our study. All cases were sent fresh, and grossly dissected, then immersed in NBF during 3 different periods then processed in a Leica ASP $6025^{\mathrm{TM}}$ automat, prior to paraffin embedding. ER(SP1), PR(1E2) and Her2(4B5) IHC were performed on all samples with a Ventana Benchmark Ultra ${ }^{\mathrm{TM}}$ automat. Percentage of positive tumor cells, intensity of staining, as well as Allred score (ER and PR) and Herceptest score (Her2) were recorded for each case and each fixation time.

Results: Mean cold ischemia time for all cases in the series was 35 minutes (18-57 minutes). Mean fixation times for Time1 (TIM1), Time2 (TIM2) and Time3 (TIM3) were 44 hours [1.86 days (1-3)], 103 hours [4.31 days (4-5)] and 182 hours [7.58 days (7-9)], respectively. At TIM1, TIM2 and TIM3 mean percentages of positive tumor cells were $76,88 \%, 75,78 \%$, and $76,33 \%$ for ER; $42,56 \%, 42,53 \%$ and $42,31 \%$ for PR; $17,06 \%$, $13,09 \%$ and $14,4 \%$ for Her2, respectively. At TIM1, TIM2 and TIM 3 mean staining intensities were 2,34, 2,31 and 2,33 for ER; $1,78,1,72$ and 1,76 for PR; $0,78,0,84$ and 0,8 for Her2, respectively. At TIM1, TIM2 and TIM3 mean Allred scores were 6.37, 6.31 and 6.30 for ER, 4.71, 4.75 and 4.82 for PR, respectively; whereas mean Herceptest scores were $0.71,0.75$ and 0.70 , respectively. Using a $10 \%$ positivity threshold, no case in our series changed ER status at the three periods. For PR, 2 cases became positive at TIM2, 1 case became positive and one case became negative at TIM3. In these cases there is a low percentage of PR positive cells (mean 16,25\%), near the $10 \%$ threshold. Change in PR status operates both ways and is most likely due to intra-tumoral heterogeneity. The 3 Her2 $3+$ cases in our series did not change status at the different periods, 1 case went from $1+$ to $2+($ HER not amplified at FISH) and 1 case went from $2+$ to 0 (equivocal HER2 status with Her2/CEN17 of 1,94) at TIM3. It is difficult to know if this change is due to prolonged fixation or intra-tumoral heterogeneity.

Conclusions: In our series, fixation times exceeding recommended periods, but less than 10 days, do not significantly affect the status of ER, PR nor Her2.

174 Follow-Up Study of 42 Patients with a Benign Intraductal Papilloma Diagnosed on Core Needle Biopsy

$C$ Diloreto, $Y$ Zhang. University of California Davis, Sacramento, CA.

Background: The debate over the management of benign intraductal papillomas diagnosed on core needle biopsy continues; some advocate for excision due to risk of atypia or malignancy, while others endorse close clinical observation. At our institution, patients with a diagnosis of benign intraductal papilloma on core biopsy are frequently offered close clinical and radiologic follow-up if the imaging studies are concordant with pathologic diagnosis. This study is to analyze our follow-up data on these patients and to determine whether surgical excision of benign intraductal papilloma is necessary. Design: Patients with a diagnosis of benign intraductal papilloma in a needle core biopsy sample and following excision were identified from our surgical pathology database from January 2000 to January 2009. The corresponding clinical and radiologic data were collected. All slides were reviewed to confirm the diagnosis of benign intraductal carcinoma. Core biopsies containing a concurrent high risk lesion and the cases with radiologic and pathologic discordance were excluded.

Results: Fifty-two cases of benign intraductal papilloma diagnosed on core needle biopsy with concordant imaging were identified. The age of the patients ranged from 22 to 88 years (mean, 54.9). Most papillomas were diagnosed by ultrasound-guided vacuum assisted core biopsies ( 26 cases, $59 \%$ ), 10 by stereotactic-guided core biopsy, 3 by MRI-guided core biopsy, and 3 by palpation-guided core biopsy. Twenty-two of 42 cases were classified as central papilloma, the remaining 20 were peripheral. Of the 42 patients, follow-up information was available in 38. During follow-up, 4 patients underwent excision because of nipple discharge at a median of 40 months, all excisions were residual benign intraductal papilloma. One patient was found to have interval growth mammographically on 60 months follow-up . Her excision showed intraductal papilloma with low grade ductal carcinoma in situ. The remaining 34 (80\%)observed patients had stable radiological findings at follow-up (26-115 months, mean 60 months). Conclusions: Close clinical and radiologic follow up is a reasonable alternative in the management of benign intraductal papillomas diagnosed on core needle biopsy, especially when ultrasound-guided vacuum assisted are used for tissue sampling. Larger studies with follow-up are needed to confirm our findings.

175 Computational Image Analysis of Nuclear Morphology Distinguishes Ductal Carcinoma In Situ from Usual Ductal Hyperplasia of the Breast

F Dong, MF Lerwill, EF Brachtel, NW Knoblauch, L Montaser-Kouhsari, NC Jones, DC Wilbur, AH Beck. Massachusetts General Hospital and Harvard Medical School, Boston, MA; Beth Israel Deaconess Medical Center and Harvard Medical School, Boston, MA. Background: Technological advances in whole slide imaging and image analysis combined with statistical modeling and pathology informatics have the potential to aid pathologists in the characterization of disease. To examine the potential value of image 
analysis in pathological practice, an automated nuclear feature extraction algorithm was developed for routine histological slides stained with hematoxylin and eosin, and the algorithm was applied to a fundamental pathological problem: the distinction of ductal carcinoma in situ (DCIS) from usual ductal hyperplasia (UDH).

Design: 96 consecutive breast core biopsy specimens with a diagnosis of DCIS or UDH from a single institution were identified. Nuclear morphometric features were extracted from regions of interest of whole slide images and quantified using the open-source image processing software FIJI (ImageJ, National Institutes of Health). Multivariate predictions of DCIS versus UDH and low grade versus high grade DCIS using extracted nuclear features were modeled by logistic regression with lasso regularization.

Results: Image processing and quantitation of select nuclear features of UDH and DCIS are depicted in Figure 1.
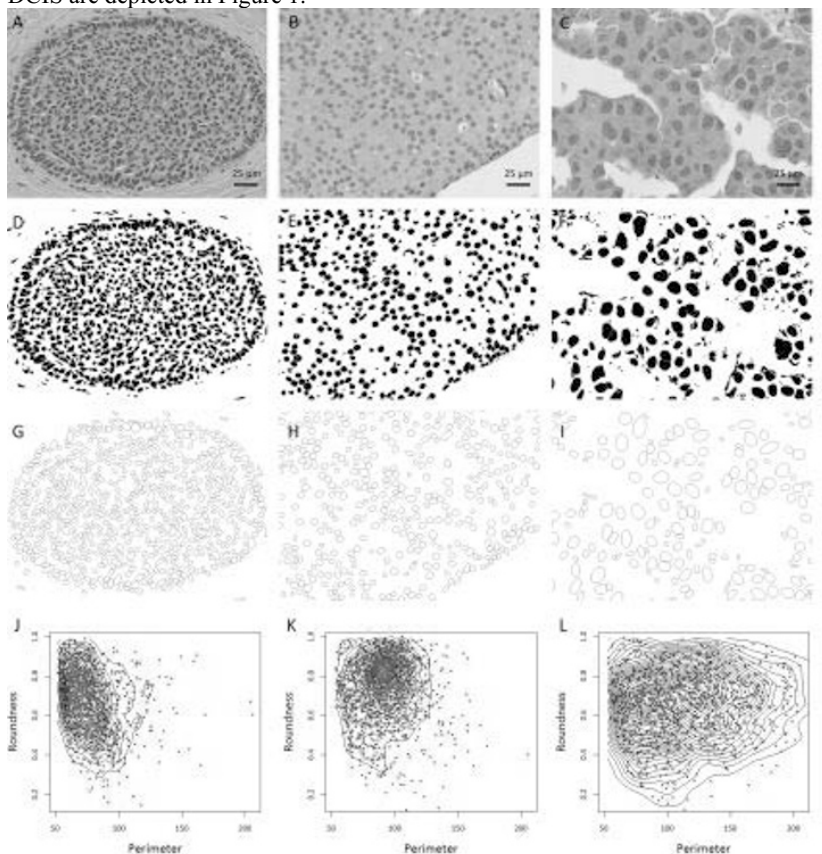

The mean and variance of multiple extracted features corresponding to nuclear size, color and shape were strongly associated with a pathological diagnosis of DCIS versus UDH and with the grade of DCIS. A logistic regression model of 20 features with 9-fold cross-validation demonstrated an ability to predict DCIS from UDH with area under the receiver operating characteristic curve of $0.950 \pm 0.025$. Furthermore, a regression model built upon a comparison of low grade versus high grade DCIS was able to associate quantitative morphometric features with estrogen receptor expression in intermediate-grade DCIS ( $\mathrm{p}=0.03$ ).

Conclusions: Digital image analysis identified morphologic features that quantitatively define UDH, low grade DCIS and high grade DCIS. These findings demonstrate the applicability of image analysis and quantitative methods as a diagnostic tool in anatomic pathology and in connecting cellular morphology with the underlying biology of disease.

176

Retrospective Analysis of Early Onset Versus Post-Menopausal Patients with Breast Cancer

EK Drinka, RL Bassett, ME Edgerton. M.D. Anderson Cancer Center, Houston, TX. Background: Breast cancer in younger patients is considered an independent predictor of poor outcome; however, disease prevalence is low in younger women. Our institution has a large cohort of patients available for review.

Design: Over 500 patients aged 20-30 years (YP) diagnosed with breast carcinoma were treated at MD Anderson during 1995-2005. These are compared with post-menopausal patients (PMP) aged 60-70 years treated during the same period. Clinicopathologic data was collected from patients' charts. Comparisons use Fisher's exact test for categorical and Wilcoxon rank-sum test for ordinal variables. Using O'Brien-Flaming boundary of Lan-DeMets spending function for interim look we established a cut-off p-value of 0.00006 for early comparison of 100 patients from each group.

Results: Average ages at diagnosis was 27.6 yrs for YP's and 64.6 yrs for PMP's. Neoadjuvant chemotherapy, adjuvant chemotherapy, and radiation therapy was given to $57.3 \%, 67.7 \%, 56.3 \%$ of YP's vs $14.6 \%, 37.5 \%$ and $52.0 \%$ of PMP's. Table 1 summarizes significant differences. Figures 1 and 2 show overall survival, and time to first recurrence/metastasis.

\begin{tabular}{|c|c|c|c|c|}
\hline بs & W & $\widehat{Y P(20-30)}$ & PMP (60-70) & p-value \\
\hline \multicolumn{5}{|l|}{ Her2neu } \\
\hline & Negative & 441 & 57 & \\
\hline & Positive & 33 & 8 & 0.00006 \\
\hline & Equivocal & 2 & 2 & \\
\hline \multicolumn{5}{|l|}{ Breast Subgroup } \\
\hline & Luminal A & 227 & 47 & \\
\hline & Luminal B & 19 & 2 & 0.00006 \\
\hline & Her2/neu + & 14 & 5 & \\
\hline & Triple Negative & 12 & 11 & \\
\hline \multicolumn{5}{|c|}{ Invasive Carcinoma Grade } \\
\hline & 1 & $\sqrt{1}$ & 9 & \\
\hline & 2 & 22 & 47 & 0.00006 \\
\hline & 3 & 772 & 30 & \\
\hline \multicolumn{5}{|l|}{ Genetic Counseling } \\
\hline & Yes & 66 & 99 & $<0.00006$ \\
\hline & No & 35 & 0 & \\
\hline
\end{tabular}
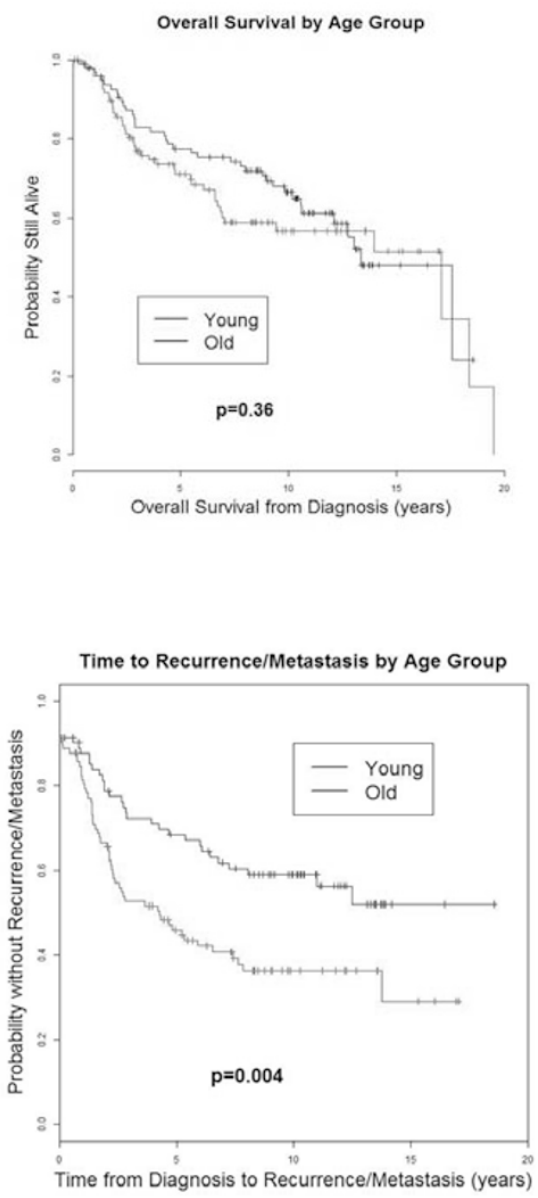

Conclusions: Early comparison shows that YP's were more likely to have Her2neu + tumors, higher grade invasive carcinomas, self diagnose, and undergo genetic counseling. Additional trends not meeting stringency for early conclusions suggest greater small vessel invasion (LVI), higher $\mathrm{N}$ stage, shorter time to recurrence, and more nonwhite patients in the YP's. Differences in overall survival at 15 years were not significant at our institution; evaluation of significance in differences prior to 10 years requires more patients.

177 Revisiting Infiltrating Epitheliosis of the Breast: A Lost Entity CEberle, E Rakha, B Weigelt, JS Reis-Filho, IO Ellis. Memorial Sloan-Kettering Cancer Center, New York, NY; University of Nottingham, Nottingham, United Kingdom. Background: Infiltrating epitheliosis (IE) is a rare complex sclerosing lesion of the breast, characterized by infiltrating ducts and duct-like structures filled up with cells with architectural and cytological patterns reminiscent of those of usual ductal hyperplasia (UDH), immersed in a scleroelastotic stroma. The pathological characteristics of this lesion remain to be fully characterized. No markers distinguishing IE from other proliferative and scleroelastotic lesions of the breast have been established. In this study we sought to define the histologic and immunohistochemical features of this unique lesion.

Design: Cases of IE were retrieved from the consultation files of one of the authors (IOE). Cases were reviewed by three pathologists, and only samples classified as IE where there was a consensus among the three pathologists were included. To characterize the immunophenotype of IE, immunohistochemistry with monoclonal antibodies against ER, CK5/6, and p63 was performed using routine methods. 
Results: Cases of IE were characterized by the presence of ductal structures filled up with cells reminiscent of those of UDH with the periphery of the lesion having jagged, irregular edges infiltrating the adjacent breast stroma and often encasing normal terminal duct-lobular units. The adjacent stroma varied from myxoid to desmoplastic to densely sclerotic. Areas composed predominantly of basal-looking cells, clear cells and squamous metaplasia were identified. IEs were associated with other epithelial proliferative lesions including classic UDH (case 2), a papillary lesion (case 7), and DCIS (case 3). A low-grade adenosquamous carcinoma was detected in two cases (cases 3 and 7). ER expression was observed in 4 cases, ranging from $5-40 \%$ of the cells. p63 was completely absent around the periphery of the epithelial structures in 2 of 7 IEs, while the remaining 5 cases showed positive, but discontinuous p63 staining. p63 and CK5/6 positivity of cells within the IE lesion ranged from $0-10 \%$ and $25-90 \%$, respectively. Conclusions: IE displays a heterogeneous epithelial phenotype. The histologic features of IE and the lack of myoepithelial cells, particularly at the periphery of the lesions, raise the concern that IE may constitute a form of low-grade infiltrating neoplasm. Further studies are required to determine whether IEs would merely constitute a form of complex sclerosing lesion of the breast or a form of low-grade carcinoma pertaining to the spectrum of low-grade adenosquamous carcinomas.

178

Establishing Recommendations for Estrogen Receptor (ER) Staining in Cytology Cell Block Material

REK Eisenberg, A Gitika, R Elyn, G Meighan, A Muzaffar, C Anibal, L Oscar, E Marcia Memorial Sloan-Kettering Cancer Center, New York, NY.

Background: FNA is a common method for diagnosis and determination of hormone receptor status in breast carcinoma (ca) metastases. ASCO/CAP guidelines for standardization, validation and interpretation of ER are established for surgical specimens. There are no recommendations for FNA cell block (CB) material.

Design: Cases of biopsy $(\mathrm{Bx})$ proven $\mathrm{ER}+$ invasive ca spanning $>1 \mathrm{~cm}$ with excision were studied. 8 cytology scrapings and $1 \mathrm{Bx}$ were performed on each ca at grossing. Scrapings were fixed in $10 \%$ neutral buffered formalin (NBF) and processed at 1,3 , $6,8,12,24,48$ and 72 hrs. CBs were prepared per protocol. Bxs were fixed in $10 \%$ NBF for 8 hrs. Histological processing included a short cycle with no NBF step. H\&E-stained slides were prepared. Slides were evaluated for cellularity ( 0 cells, $<20$ or $>20$ ) prior to ER staining. ER \% and intensity were recorded. CBs processed within $2 \mathrm{hrs}$ of intended time point (for $<24 \mathrm{hrs}$ ) and within $6 \mathrm{hrs}$ (for $\geq 24 \mathrm{hrs}$ ) were included. Cases studied met both cellularity ( $<20$ or $>20$ cells $)$ and time point inclusion criteria. Agreement between Bxs and CBs in \% staining was assessed using concordance correlation coefficients (CCC)

Results: 16 pts with ER+ invasive ca were prospectively identified. All pts had excision at our institution over 10 months (6/12-4/13). Pts were female with mean age 59 (range 31-97). Surgical specimens included 8 excisions and 8 mastectomies. Mean tumor size was $2 \mathrm{~cm}$ (range 1-4.8 cm). The cases included 11 invasive ductal ca, 3 invasive lobular ca, 1 tubular ca, and 1 mucinous ca. Mean CBs that met inclusion criteria per time point was 7 (range 4-10). 12 Bxs met inclusion criteria. All cases which stained for ER were moderate to strong intensity. The mean \% of ER+ stained cells on CB was 74 (range $0-100$ ) and on Bx was 95 (range 80-100). Per time point, mean \% staining was 80 (1), 71 (3), 70 (6), 77 (8), 90 (12), 72 (24), 68 (48), and 62 (72). 6\% of CBs were ER-. Exact agreement in percent staining between CBs and Bxs by CCC was poor (range $-0.27-0.58$ ) at all 8 time points. However, detection of any ER+ staining from CB was very high and agreed with $\mathrm{Bx}>86 \%$ except at $72 \mathrm{hrs}(75 \%)$

Conclusions: $\mathrm{CB}$ material fixed in NBF for only $1 \mathrm{hr}$ can be used to assess ER staining by IHC. Although the reproducibility of the exact $\%$ of tumor cells staining was poor between $\mathrm{CB}$ and $\mathrm{Bx}$ material at a given time point, the detection of any positive staining on $\mathrm{CB}$ agreed with the Bx over $88 \%$ at all time points except at $72 \mathrm{hrs}$.

179 ERCC1 Expression in Breast Ductal Cancers in African American Women

AK Esnakula, LJ Ricks-Santi, Y Kanaan, TJ Naab. Washington University, St Louis, MO; Hampton University, Hampton, VA; Howard University Hospital, Washington, DC. Background: Excision Repair Cross Complementing group 1 protein (ERCC1) functions in repairing damage induced by platinum-based chemotherapy. Absence of ERCC1 expression has been associated with response to these agents. The objective of our study was to correlate the immunohistochemical expression of ERCC1 in the four major subtypes of breast carcinoma (luminal A, luminal B, HER2, and Triple Negative) with other clinicopathological factors.

Design: Tissue microarrays (TMAs) were constructed from formalin-fixed paraffin embeded tumor blocks from primary ductal breast carcinomas in 203 African-American females. Two separate $1 \mathrm{~mm}$ cores represented each case. The polymer-HRP system was utilized for immunostaining. Five micrometer sections are stained with a mouse monoclonal antibody against ERCC1 (8F1, Diagnostic Biosystems, Pleasanton, CA). The sections are evaluated for the intensity of nuclear reactivity (2-3) and the percentage of reactive cells; and an $\mathrm{H}$-score is derived from the product of these measurements. Cases are categorized as having negative/weak (score $\leq 10$ ) or moderate/strong (score $>10$ ) ERCC1 expression. Bivariate analysis is done via $\chi 2$ analysis and survivability data was calculated via the generation of Kaplan-Meier curves (SPSS v19). Statistical significance was assumed if $\mathrm{P}<0.05$.

Results: ERCC1 expression was statistically associated with lower grade tumors $(\mathrm{p}=0.027)$, HER 2 positivity $(\mathrm{p}=0.011)$, lymph node negativity $(\mathrm{p}=0.003)$, and overall survival $(\mathrm{p}=0.019)$

Conclusions: In our study, ERCC1 expression was linked to lower grade luminal carcinomas and HER 2 positive tumors indicating possible limited efficacy of platinumbased therapy in these groups. In our study, 31 of $62(50 \%)$ cases showed no ERCC1 expression. This suggests that platinum based chemotherapy may be effective in this group.
BTG2 Loss Is Highly Associated with Triple Negative BreastCancers in African American Women

AK Esnakula, LJ Ricks-Santi, TM Karve, Y Kanaan, TJ Naab. Washington University, St Louis, MO; Hampton University, Hampton, VA; Howard University Hospital, Washington, DC

Background: Inactivation of tumor suppressor genes often plays a significant role in breast carcinogenesis and tumor progression. The B-cell translocation gene-2 (BTG2), a gene induced by $\mathrm{p} 53$, belongs to an anti-proliferative gene family. The goal of our study is to correlate BTG2 immunohistochemical expression in the four major subtypes of breast ductal carcinoma (Luminal A, Luminal B, HER2, and Triple Negative) with other clinicopathological factors.

Design: Tissue microarrays (TMAs) were constructed from optimally-fixed formalinfixed, paraffin-embedded tumor blocks from primary breast ductal carcinomas in 203 African-American females. Two separate $1 \mathrm{~mm}$ cores represented each case. The polymer-HRP system was utilized for immunostaining. Five micrometer sections are stained with a mouse monoclonal antibody against BTG2 (Genway Biotech, San Diego, CA). The sections are evaluated for the intensity of nuclear reactivity $(0-3)$ and the percentage of reactive cells; and an $\mathrm{H}$-score is derived from the product of these measurements. Cases are categorized as having negative/weak (score $<10$ ) or positive (score $>10$ ) expression. Bivariate analysis is done via $\chi 2$ analysis and survivability data is calculated via the generation of Kaplan-Meier curves (SPSS v19). Statistical significance was assumed if $\mathrm{P}<0.05$.

Results: BTG2 expression is significantly associated with ER positivity $(\mathrm{p}<0.001)$, PR positivity ( $p=0.001)$, Luminal A and B breast cancer $(p<0.001)$. Loss of BTG2 is significantly associated with triple negative subtype $(p<0.001)$. No correlation of BTG2 expression is seen with survival.

Conclusions: Triple negative breast cancers are associated with aggression, poor prognosis and have an increased incidence in younger African American women. In human triple-negative breast cancer cell lines, BTG2 has been shown to inhibit proliferation and induce apoptosis. Our study demonstrated marked decrease/absence of BTG2 expression in the triple negative subtype, supporting the important role that BTG2 silencing plays in disease progression. Recent in vitro and in vivo studies have shown that HER2 inhibitors, such as lapatinib, negate breast cancer progression due to loss of BTG2, thereby, indicating potential therapeutic efficacy in treating tumors with BTG2 loss.

181 GATA3, Mammaglobin, and GCDFP-15 Expression in Subtypes of Breast Ductal Carcinoma in African American Women

AK Esnakula, LJ Ricks-Santi, Y Kanaan, TJ Naab. Washington University, St Louis, MO; Hampton University, Hampton, VA; Howard University Hospital, Washington, DC. Background: Mammaglobin and GCDFP-15 are often used to identify breast origin in metastases due to robust diffuse expression in mammary tissue. More recently, GATA3 has been found to be selectively expressed in breast cancer. The goals of our study were to evaluate the immunohistochemical expression of these three antibodies in ductal breast cancers from African American women, to compare expression in the four major breast cancer subtypes (Luminal A, Luminal B, HER2, Triple Negative), and to correlate the findings with other clinicopathologic features.

Design: Tissue microarrays were constructed from tumor blocks from primary breast carcinomas in 203 African-American females. Two separate $1 \mathrm{~mm}$ cores represented each case. The polymer-HRP system was utilized for immunostaining. Five micrometer sections are stained with mouse monoclonal antibodies against GATA3 (L50-823, Biocare Medical), mammaglobin (304-1A5, Dako), and GCDFP-15 (23A3, Cell Marque). The sections are evaluated for intensity of cytoplasmic reactivity $(0-3)$ and percentage of reactive cells; an $\mathrm{H}$-score is derived from the product of these measurements. Cases are categorized as having negative/weak (score $\leq 10$ ) or moderate/ strong (score $>10$ ) expression.

Results: GATA3 expression shows association with lower grade $(\mathrm{p}<0.001)$, ER positivity ( $\mathrm{p}<0.001)$, PR positivity ( $<<0.001)$, and Luminal A and B subtypes $(\mathrm{p}<0.001)$. Mammaglobin expression is associated with lower grade $(\mathrm{p}=0.031)$, ER positivity $(\mathrm{p}=0.038)$, PR positivity $(\mathrm{p}=0.022)$. GCDFP-15 expression shows no statistical significance with any parameter, being expressed in only 14 cases.

Conclusions: GATA3 and mammaglobin demonstrate expression predominantly in luminal breast cancers, but are expressed in some TN breast cancers $(24 / 6239 \%$ and $9 / 6214 \%$, respectively) so they may be useful in confirming breast origin in metastases in this TN subset. GATA3 is expressed in $73 \%$ of cases and mammaglobin in $61 \%$. Surprisingly, GCDFP-15 is expressed in only $8 \%$ of cases.

\section{Enrichment for PI3K Pathway Mutations in Metastatic Low} Proliferative Rate ER+ Breast Cancers

MV Estrada, MG Kuba, JM Giltnane, JM Balko, IA Mayer, V Abramson, I Meszoely, C Vnencak-Jones, CL Arteaga, ME Sanders. Vanderbilt University, Nashville, TN. Background: The PI3K pathway is the most frequently mutated pathway in breast cancer (BC). Data from The Breast Cancer Genome Atlas Network indicates these mutations occur across all BC subtypes (36\%), with the highest frequency in luminal $\mathrm{A} / \mathrm{ER}+\mathrm{BC}(\sim 45 \%)$. We previously identified increased mutation frequency in primary invasive lobular carcinomas (ILC) and carcinomas with lobular features. We have reported an association between PIK3CA mutations and triple negative (TN)BC with metaplastic features. Several studies report higher prevalence of PI3K pathway mutations in metastatic BC (42-53\%)

Design: We characterized the histopathologic features of 350 primary, 27 recurrent and 53 metastatic $\mathrm{BC}$ with known hormone receptor and HER2 status previously screened for 18 hotspot mutations in the PIK3CA (14), PTEN(3) and $A K T(1)$ genes utilizing a SNaPshot multiplex platform developed at our institution. Primary tumors were typed 
and graded according to $\mathrm{WHO}$ and $\mathrm{AJCC}$ criteria. Relationship of mutation frequency to histopathologic features, hormone receptor expression and HER2 status was examined. Slides were reviewed by three breast pathologists for concordance.

Results: The overall PI3K pathway mutation rate was $26.5 \%(114 / 430)$. The distribution of mutations was: PIK3CA (104), AKTI (9) and PTEN (1). $25.7 \%$ of primary tumors and $18.5 \%$ of local recurrences vs $35.8 \%$ of metastatic tumors carried mutations. Mutations were more frequent in ER+ $(35.4 \%)$ than in HER2+ $(14.7 \%)$ and TN $(9.8 \%)$ BC. Furthermore, mutation frequency in ER+ and TNBC metastases was higher than that observed in primary tumors $(42.1 \%$ vs. $35.3 \%)$ and ( $28.6 \%$ versus $7.8 \%)$, respectively. Among 350 primary tumors, mutation frequency was inversely correlated with histologic grade $(30.4 \% / 51.7 \%$ low/intermediate vs. $13 \%$ high; $\mathrm{p}<0.0001)$ and proliferative rate (low $37 \%$ vs. high $15 \% ; \mathrm{p}=0.0001$ ) and further enriched among ILC and tumors with lobular features (No special type $20.2 \%$ vs. ILC/lobular features $38 \% \mathrm{p}=0.0001$ ). $93 \%$ of mutations in $\mathrm{ER}+$ metastases occured in tumors with a low proliferative rate. The 2 metastatic TNBC with mutations had a high grade DCIS-like appearance with central necrosis. There were no other recurrent histologic features predictive of a PI3K pathway mutation in the primary tumors or metastases.

Conclusions: The frequency of PI3K pathway mutations is greater in metastatic ER+ $\mathrm{BC}$ and TNBC than that seen among primary tumors of these subtypes. Among ER+ $\mathrm{BC}$, a low proliferative rate and/or lobular features are most predictive of a mutation in both primary tumors and metastases.

\section{Beta-2 Adrenergic Receptor-Expressing Macrophages in Breast} Carcinomas: A Potential Therapeutic Target?

$V$ Farahmand, B Julianne, C Steven, G Patricia, PS Sullivan. University of California, Los Angeles, Los Angeles, CA.

Background: Recent studies suggest breast cancer progression may be regulated by the sympathetic nervous system via beta-adrenergic recruitment of macrophages and that this pathway for disease progression may be targeted by beta-adrenergic receptor antagonists. We sought to assess the macrophage density and beta adrenergic receptor co-expression in human breast cancer tissues and to correlate these findings with clinical parameters.

Design: Archival breast cancer tissue and clinical parameters were collected from 99 women at a single institution. Formalin-fixed paraffin embedded tumor sections underwent immunohistochemical staining for CD68 and beta2 adrenergic receptor (B2). Macrophage density and B2 co-expression within the tumor and along the tumor edge were assessed by 2 independent observers. Macrophage density was expressed as the number of CD68-positive cells per 10 high power fields. B2 co-expression was assessed by estimating the percentage of macrophages with B2 staining.

Results: CD68 and B2 scoring had good reproducibility. Increased intratumoral macrophage density was associated with increased intratumoral B2 co-expression $(\mathrm{p}=0.02)$, ER negativity $(\mathrm{p}=0.02)$, increased Ki-67 index $(\mathrm{p}<0.0001)$, HER2 overexpression, high grade, and T2 tumors. No association was seen with lymph node status. Increased edge macrophage density was also associated with increased edge B2 co-expression $(\mathrm{p}=0.07)$.

Conclusions: Increased intratumoral and edge macrophages are associated with increased B2 expression and poor prognostic markers in breast cancer. Findings highlight the association of the breast tumor microenvironment with the sympathetic nervous system and support the potential role for beta-adrenergic receptor-targeted therapy in breast cancer.

184 Did OncoType DX Recurrence Score Accurately Predict the Risk of Recurrence and Correctly Guide Who Can Avoid Chemotherapy in Breast Cancer? A 10 Year Clinical Follow-Up Study

$V$ Farahmand, SK Apple. University of California, Los Angeles, Los Angeles, CA. Background: The 21-gene Oncotype DX recurrence score (RS) assay predicts the risk of recurrence and benefit from chemotherapy in estrogen receptor (ER) positive, node negative, and, more recently, limited node-positive breast cancer. The RS is divided into low (L), intermediate (I), and high $(\mathrm{H})$ corresponding to a likelihood of recurrence within 10 years of the initial diagnosis. Clinicians utilize Oncotype DX to guide treatment, particularly whether to add chemotherapy to endocrine therapy. We sought to investigate if Oncotype DX RS accurately predicts the rate of recurrence with respect to each category.

Design: A cohort of 236 patients tested for Oncotype DX from 2003 to 2013 was studied retrospectively. The average follow-up was 28 months with a range of 1 to 102 months. Examined were age, ER, progesterone receptor (PR), HER-2/neu, Ki-67, tumor size and grade, lymph node status, death, recurrence, metastases, and chemotherapy status. Results: LRS, IRS and HRS comprised $65 \%, 29 \%$, and $6 \%$ of the 236 cases studied. We found $98.7 \%$ were $\mathrm{ER}+, 95 \%$ were $\mathrm{PR}+$, and $99.5 \%$ were Her- $2 /$ neu negative; Grade 1 , 2 and 3 cases were $38 \%, 51 \%, 11 \% ; 97 \%$ were T1 or T2, while $3 \%$ were T3; and $17 \%$ had positive lymph nodes. $39 \%$ of IRS and $73 \%$ of HRS received chemotherapy. Of the 15 HRS patients, all had negative nodes, $73 \%$ had Ki- $67 \geq 20 \%, 33 \%$ were PR-, and $7 \%$ were Her-2/neu positive. Discordance rate between IHC and RT-PCR by Oncotype DX for ER, PR and Her-2/neu was low: $1 \%, 9 \%$ and $0 \%$ respectively. No deaths occurred in all patients studied, while $0.8 \%(2 / 236)$ had recurrence/metastasis. One LRS had recurrence in 63 months and one IRS who received chemotherapy had bone metastasis in 48 months. Both patients had grade 2, ER + , Her-2/neu negative and low Ki-67 tumors, while one was PR+ with one node + and the other was PR- with 8 negative nodes.

Conclusions: The addition of chemotherapy in the treatment regimen for node,$+ \mathrm{Her}-2 /$ neu+, and PR negative tumors may be beneficial regardless of RS scores. Ki- 67 of $\geq 20 \%$ and negative/weak PR are more likely to have HRS. There is a high concordance rate between RT-PCR and IHC of ER, PR, and Her-2/neu. While Oncotype DX accurately predicted $99.4 \%$ for the low RS to avoid receiving chemotherapy, the utility of Oncotype $\mathrm{DX}$ is questioned since outcome and treatment are not dramatically different from what is expected based on standard morphoimmunohistologic variables.

185 Clinical-Pathologic Significance of Androgen Receptor (AR) Expression in Hormone Receptor (HR) Negative Breast Cancer

D Farnell, G Pond, S Mukherjee, M Levine, A Bane. McMaster University, Hamilton, Canada.

Background: A subset of HR (estrogen receptor and progesterone receptor) negative breast cancers, encompassing both triple negative (TN) and HER2 over-expressing molecular subtypes have been demonstrated to express the AR. The clinical significance of AR expression in these poor prognostic subtypes of breast cancer however, is poorly characterized. In this study we examined the expression of AR in an historical cohort of HR negative breast cancers and correlated AR expression with clinical-pathologic tumor characteristics and patient outcome.

Design: $130 \mathrm{HR}$ negative primary invasive breast cancers diagnosed and treated between 2004-2007 had formalin fixed paraffin embedded (FFPE) tumor blocks available for tissue microarray (TMA) construction. On the basis of IHC expression of ER, PR, HER2, EGFR, CK5/6, and AR the tumors were classified as HER2 over-expressing or $\mathrm{TN}$ and stratified as AR positive (Allred score $>5$ ) or AR negative (Allred score $<5$ ). A centralized pathology review was additionally performed on one full face section of each tumor by an expert breast pathologist who was unaware of the molecular subtype at the time of review. Kaplan-Meier methods were used to estimate overall survival at 5 years. Fisher's exact, Wilcoxon rank sum and log-rank tests were used to compare tumor characteristics and overall survival between subtypes.

Results: 107 (82.3\%) of the 130 HR negative tumors were classified as TN while 23 (17.7\%) were HER2 over-expressing, and 26 (20.0\%) expressed AR. AR expression was observed in $15 \%$ of TN and $43 \%$ of HER 2 overexpressing tumors $(\mathrm{p}=0.004)$. When compared to AR negative tumors, patients with AR positive tumors were more likely to be older age at diagnosis; mean age $69.85 \mathrm{yrs}$ vs $61.2 \mathrm{yrs}, \mathrm{p}=0.023$. There was no significant difference between the AR positive and negative tumors with regards to tumor grade $(\mathrm{p}=0.13)$, stage $(\mathrm{p}=0.85)$, overall survival $(83.3 \%$ vs $73.9 \%$ at 5 years, $\mathrm{p}=0.56$ ) or event-free survival $(65.6 \%$ vs $68.4 \%$ at 5 years, $\mathrm{p}=0.85)$, nor was there an interaction with HER2 over-expression observed.

Conclusions: AR expression is observed in approximately $20 \%$ of HR negative breast cancers and correlated with older age at diagnosis and HER2 overexpression. No significant differences in pathological tumor characteristics or patient outcome are observed for AR + HR- tumors as compared to AR- HR-tumors irrespective of whether the tumors are TN or HER2 over-expressing.

186 Effectiveness of Core Biopsy for Screen-Detected Breast Lesions $<10 \mathrm{~mm}$. Implications for Surgical Management

G Farshid. BreastScreen South Australia, Wayville, South Australia, Australia; Adelaide University, Adelaide, South Australia, Australia.

Background: Technical advances have improved the ability to discern ever smaller mammographic lesions. In the context of population based screening accurate sampling of these lesions by percutaneous biopsy is crucial in limiting the reliance on surgical biopsies.

Design: Women undergoing core biopsy between Jan 1997-Dec 2007 for lesions $<10 \mathrm{~mm}$ are included. Patient demographics, imaging features and final histology were tabulated. Performance indices and the incidence of missed cancers, delays in surgery or cancers missing in the surgical specimen were evaluated.

Results: This audit includes 803 lesions $<10 \mathrm{~mm}$. Core biopsy was benign in 395 $(49.2 \%)$, malignant in $300(37.4 \%)$, inadequate in $25(3.1 \%)$, atypical in $60(7.5 \%)$ and suspicious in $23(2.9 \%)$. Based on core histology 345 women $(43.0 \%)$ were immediately cleared of malignancy and $300(37.4 \%)$ were referred for definitive cancer treatment. 157 women $(19.6 \%)$ required diagnostic surgical biopsy due to indefinite results or radiologic-pathologic discordance and one woman $(0.1 \%)$ needed further imaging. $46(29.3 \%)$ open biopsies were malignant. The positive predictive value of malignant core biopsy was $100 \%$. Nine of 395 cases with benign cores were ultimately malignant, yielding a negative predictive value for benign core results of $97.7 \%$ and a false negative rate of $2.6 \%$. In 8 of 157 (5.1\%) women advised to undergo surgical biopsy, the lesion could not be visualized subsequently for peroperative localization and surgery was not undertaken. Another woman had surgery but a lesion was not found. At a mean follow up of 41.8 months (range 10-68 mths) all are diseaase free. In 12 of $300(4.0 \%)$ women with malignant cores biopsies, excision specimens did not contain residual malignancy. In each case review of the cores confirmed malignancy and localization clips, the injected carbon ink track or biopsy site changes were found. No instances of local recurrence have occurred during the mean follow up of 42 months (range 19-78 mths). Excessive delays in surgery due to complications of core biopsy were not reported.

Conclusions: Even at this small size range, core biopsy evaluation of screen-detected breast lesions is highly effective, obviating the need for surgical biopsy in $43.0 \%$ of patients and guiding definitive, one stage cancer surgery in a further $37.4 \%$. A lesion miss rate of $3.1 \%$ and under-representation of subsets of lesions on core samples highlight the continued need for multidisciplinary collaboration and selective use of surgical biopsy. 
187 Menacalc, a Quantitative Method of Metastasis Assessment, as a Prognostic Marker for Axillary Node-Negative Breast Cancer

CL Forse, S Agarwal, D Pinnaduwage, F Gertler, J Condeelis, J Lin, XXue, TE Rohan, DL Rimm, IL Andrulis. University of Toronto, Toronto, ON, Canada; Yale University, New Haven, CT; Massachusetts Institute of Technology, Cambridge, MA; Albert Einstein College of Medicine, Bronx, NY

Background: Mena is an actin regulatory protein with multiple splice variants which has been shown to promote breast tumor invasion depending on the isoform expressed. Previous work (Agarwal et al., 2012) demonstrated that Mena ${ }^{\text {calc }}$, an immunofluorescence-based, quantitative method which subtracts the protein expression of the non-invasive Mena isoform (Mena11a) from total Mena expression, was correlated with poor disease-specific survival. Our goal was to determine if Mena ${ }^{\text {calc }}$ could be used as an independent prognostic marker for axillary node-negative (ANN) breast cancer. Design: Tissue microarrays were constructed using tumor specimens prospectively collected from 406 breast cancer patients that were enrolled at eight Toronto hospitals between 1987-1996. Patients were stratified into four quartiles (Q1-Q4) based on Mena $^{\text {calc }}$ score. Analysis of the association of overall survival (OS) with marker status was done by the log-rank test with Kaplan-Meier survival curves. Mena ${ }^{\text {calc }}$ status was defined as 'Higher' if the Mena ${ }^{\text {calc }}$ score fell within Q3 or Q4 and 'Lower' if the score fell within Q1 or Q2. Multivariate OS analysis to assess the contribution of Mena ${ }^{\text {calc }}$ in addition to HER2 status and clinical-pathologic tumor variables was conducted using the Cox proportional hazards model. Relative risks (RR) for each factor were estimated by the hazard ratio in the Cox proportional hazards model in both univariate (UV) and multivariate (MV) analyses.

Results: The ANN cohort varied considerably in tumor size, histological grade, nuclear grade and patient age. 58 deaths were noted during follow-up (minimum and median follow-up 56.1 and 96.5 months respectively). Higher Mena ${ }^{\text {calc }}$ quartiles were associated with decreased OS in UV analysis (Log-Rank $P=0.0251$; UV $P=0.0247$, $\mathrm{RR}(\mathrm{CI})=1.82(1.07,3.10))$. Higher Mena ${ }^{\text {calc }}$ was associated with decreased OS in MV analysis $(P=0.0293, \mathrm{RR}(\mathrm{CI})=2.01(1.07,3.77))$ independent of HER2 status, tumor size, hormone receptor status, nuclear grade, patient age and lymphatic invasion. Decreased OS was preserved when the MV model was applied to a subset of the patient population which did not receive hormone or chemotherapy ( $=143, P=0.0090$, RR $(\mathrm{CI})=3.77(1.39,10.21))$

Conclusions: High Mena ${ }^{\text {calc }}$ levels are associated with decreased overall survival of ANN breast cancer patients. Mena ${ }^{\text {calc }}$ may serve as a useful independent prognostic biomarker for this patient population.

188 Collagen 11 Alpha 1 a Better Marker for Breast Tumor Invasiveness Than Myoepithelial Cell Markers

J Freire, S Pereda, C Caballero, J Rodriguez-Parra, A Azueta, P Garcia-Arranz, J Gomez-Roman. Hospital Universitario Marqués de Valdecilla, Santander, Spain.

Background: Accurate diagnosis of invasive breast lesions, when analyzed by Core Needle Biopsy (CNB), may suppose, in certain situations, a major challenge for the pathologist. Various markers of invasiveness such as laminin, S-100 protein, myosin heavy chain for smooth muscle or calponin have been described along the last years, however none of them is completely reliable.. The use of a specific marker of the infiltrating tumor microenvironment seems vital to support the diagnosis of invasive against in situ lesions. At this point, collagen, type XI, alpha 1 (COL11A1), might be helpful in this situation since it has been described associated to cancer associated fibroblasts (CAFs) in other tumors such as lung, pancreas, or colorectal carcinomas. Previous studies of our group showed that COL11A1 expression presents a great sensitivity in determining the invasiveness of breast tumors. In this retrospective study we demonstrate that the use of COL11A1 would have changed from In situ to invasive diagnosis of CNB.

Design: Immunohistochemistry of COL11A1 (Clone 1E8.33, Oncomatrix) was performed in formalin fixed, paraffin embedded Core Needle Biopsy (CNB) samples of 39 patients with pathological diagnosis of Ductal Carcinoma In Situ. All samples were diagnosed using conventional tools and immunostaining panels for myoepithelial cells. Twenty one of these samples showed infiltration in subsequent surgical biopsy. Results: Our results showed a significant difference $(p<0.001)$ in COL11A1 expression between lesion with infiltrative surgical biopsy (18 out of 20 positive) and the rest of in situ lesions (2 out of 19). Positive staining was clearly observed in fibroblast's cytoplasm of tumor stroma of the invasive lesions, whereas signal was not detected in In Situ confirmed lesion. The levels of both sensitivity and specificity where $90 \%$. Conclusions: These data confirm that stromal fibroblast overexpression of COL11A1 is a better marker of infiltration when compared with other reported markers, with a very high level of sensitivity and specificity. COL11A1 should be added to the immunohistochemical panel in order to diagnose $\mathrm{CNB}$ of infilitrative breast tumors.

189 Role of Src/Ezrin in Angio- and Lymphangiogenesis in Breast Cancer: Potential for a New Targeted Therapy

A Ghaffari, S Varma, A Szeto, V Hoskin, S Sengupta, Y Madarnas, B Elliott. Queen's University, Kingston, ON, Canada; Cancer Research Institute, Kingston, ON, Canada. Background: Overexpression of ezrin, a membrane-cytoskeletal crosslinking protein, has been associated with lymphovascular invasion (LVI), positive lymph node (LN) status, and poor outcome in various cancers including breast. One of the mechanisms of ezrin-associated changes is by activation of Src. The role of Src-ezrin interaction in lymphatic invasion has not been studied to date.

Design: In a tissue microarray of 63 invasive breast cancers (BC), immunofluorescencebased automated quantification analysis (AQUA) was carried out for Src and Ezrin expression. Data on lymphovascular invasion was collected from the Pathology reports using whole tissue sections. To understand the mechanistic aspects, we examined the role of Src/ezrin in tumoral Angio- and lymphangiogenesis using highly invasive human MDA-MB-231 breast carcinoma cell line and xenograft model.

Results: Significantly higher levels of both ezrin and Src were seen in triple negative breast cancers $(\mathrm{p}=0.034 \& 0.003$, respectively). In addition, higher Src AQUA scores were seen in high grade tumors $(\mathrm{p}=0.027)$. The LVI+ cases showed higher levels of both ezrin and Src compared to LVI- cases ( $\mathrm{p}=0.024 \& 0.031$, respectively). Human lymphatic endothelial cells co-cultured with MDA-MB-231 cells expressing constitutively active Src (MDASrc or CA-Src) showed higher migration, tube formation and permeability compared to those cultured with ezrin knockdown MDASrc (ezrin KD) cells. Conditioned media from MDASrc induced a robust endothelial branching response in ex vivo aortic ring assay, whereas this effect was blocked in ezrin KD cells. Xenografts of matrigel plugs containing ezrin KD both in wtMDA231 and MDASrc cells revealed significant reduction in tumour induced angio and lymphangiogenesis. Analysis of downstream targets in the Src-induced angiogenesis pathway showed that ezrin is required for Src-induced Stat 3 activation leading to VEGF-A/-C expression. Conclusions: Our study has shown a novel function for ezrin in the regulation of tumorinduced lymphangiogenesis and hence, prognosis in early stage BC. Current clinical trials on Src inhibitors in $\mathrm{BC}$ have failed to show therapeutic benefit. However, our study suggests that combination therapy with newer ezrin and src inhibitors together may have a potential therapeutic advantage in BC. (Supported by CIHR, BCAK, and Terry Fox Foundation in partnership with CIHR).

190 Invasive Bilateral Breast Cancer

$R$ Ghai, B Mahon, VB Reddy, P Gattuso. Rush University Medical Center, Chicago, IL. Background: The aim of this study was to determine whether the breast cancer biomarkers vary among bilateral breast cancers diagnosed synchronously.

Design: Synchronous bilateral breast cancer was defined as bilateral breast cancer diagnosed concurrently or within 6 months of each other. We compared the biomarker status of these cases and evaluated if the variation was related to morphologic features and the grade of the tumor.

Results: Between 1998 and 2012, bilateral breast cancer cases diagnosed synchronously were retrieved from our pathology archives. The estrogen receptor (ER), progesterone receptor (PR) and Her-2/neu results of these tumors was classified as either positive or negative. Her-2/neu status by immunohistochemistry and fluoresnce in-situ hybridization was evaluated. A total of 69 cases were diagnosed with synchronous invasive bilateral breast cancer. The age ranged from 27 to 76 years with an average age at diagnosis of 56 years. Majority of the patients were multiparous and $79 \%$ were post menopausal. More than half of the patients $(55 \%)$ had at least one first degree relative with a history of breast or ovarian cancer and $9 \%$ of the cohort had a deleterious mutation in BRCA1 or BRCA2. Infiltrating ductal carcinoma (74\%) was most common followed by infiltrating lobular carcinoma (21\%). Associated ductal carcinoma in-situ was identified in 53\% of cases and $19 \%$ of cases had multifocal disease. $35 \%$ of the patients had lymph node involvement with a higher clinical stage of disease. 8 of the 69 cases $(12 \%)$ were identified with different biomarker results. The discordant results were related to tumor grade (4 cases) and morphology (4 cases)

Conclusions: Despite identical hereditary and environmental exposure, some patients develop synchronous breast cancers wth discordant biomarkers suggesting other factors may influence tumor characteristics. Prognosis maybe determined by the tumor with worst prognosis and hence biomarker studies should be performed on bilateral tumors as a small number of cases exhibit different biomarker expression which can affect treatment strategy.

\section{Fibroadenomas Presenting as Suspicious MRI Findings:} Correlation of Pathologic Features with Diffusion Weighted Imaging

NV Giannakopoulos, X Chai, H Rahbar, S Parsian, C-L Liu, SC Partridge, MH Rendi. University of Washington, Seattle, WA.

Background: Breast magnetic resonance imaging (MRI) is increasingly utilized for women at high risk for breast cancer. However, conventional MRI features suggesting malignancy do not always correlate with pathologic findings, with many benign lesions undergoing unnecessary biopsy. Diffusion weighted MRI (DW-MRI) is a functional MR technique that measures the apparent diffusion coefficient (ADC) of tissue, which is related to the mobility of water molecules in vivo and indirectly, the histologic characteristics of the tissue. DW-MRI studies of the breast have demonstrated significantly lower ADCs for breast carcinomas than for benign breast lesions or normal tissue. Fibroadenomas (FAs) are one of the most common false-positives on conventional breast MRI. Our prior work has shown that while many FAs demonstrate an ADC above the malignancy ADC cutoff, a significant number exhibit low ADCs and present a challenge for implementing a useful diagnostic ADC threshold. In this study we compare the histologic characteristics of FAs presenting as false-positive lesions on both conventional breast MRI and DW-MRI with FAs that are negative on DW-MRI. We aim to identify histologic correlates that explain the anomalous imaging findings. Design: After IRB approval, we retrospectively evaluated DW-MRI scans in consecutive women with suspicious findings on clinical breast MRI from 10/2005 to 12/2008 that were subsequently biopsied and showed FA. ADC measures were calculated on DWMRI, and each case was pathologically reviewed for stromal type, stromal cellularity, epithelial proliferation and presence of inflammation. Histologic findings were then correlated with lesion ADC measurement using Wilcoxon Rank-Sum test.

Results: Twenty-six FAs in 25 women were included. ADC values were significantly lower in FAs with increased epithelial proliferation $(\mathrm{p}=0.03)$ and in FAs with higher density collagenous stroma ( $\mathrm{p}=0.03$, Wilson's exact test). There was no correlation between ADC value and stromal cellularity, presence of inflammatory cells, or the stromal:epithelial ratio. 
Conclusions: We show that epithelial proliferation and collagenous stroma in an FA are two histologic features that are significantly correlated with false positive ADC measures on DWI. These data contribute to a better understanding of the histologic features of false positive lesions on functional MRI. Further study of this area may ultimately decrease the number of biopsies performed for false-positive MRI findings and contribute to improved radiologic-pathologic concordance for non-malignant lesions.

\section{2 \\ Smad2}

H Gilmore, P Bomeisl, C Thompson, V Varadan, M Kristy, L Harris. University Hospitals Case Medical Center, Cleveland, $\mathrm{OH}$

Background: The majority of breast cancer (BC) deaths remain a result of metastatic disease. While metastasis is the ultimate stage of tumor progression, the mechanisms are still largely unknown. About $15 \%$ of all metastatic $\mathrm{BC}$ leads to symptomatic brain metastases. There is some evidence that TGF- $\beta$, a growth factor known to have an essential role in metastasis, and downstream signaling via Smad 2 phosphorylation, may mediate metastasis of BC to the brain. However, the expression level of phospho-Smad2 (pSmad2) in brain metastases from BC has not been well studied.

Design: Tissue microarrays (TMAs) were constructed from 69 brain metastases from 61 individual patients with $\mathrm{BC}$. Also included were matched primary breast tumor $(\mathrm{N}=20)$ and other sites of metastasis $(\mathrm{N}=11)$ if available. Immunohistochemistry for antibodies specific for pSmad2, ER, PR, and HER2 was performed on $4 \mu \mathrm{m}$ tissue sections. Results of $\mathrm{pSmad} 2$ staining were recorded using percentage nuclear staining $(0=$ none, $1=1-25 \%, 2=26-50 \%, 3=51-75 \%, 4=76-100 \%)$ and intensity $(0=$ none, $1=$ weak, $2=$ moderate, $3=$ strong) that were multiplied to give an $\mathrm{H}$-score. ER, PR and HER2 were scored according to current ASCO/CAP guidelines. Differences in $\mathrm{pSmad} 2$ staining were assessed using a Wilcoxon rank test.

Results: Overall, 69 of 71 brain metastases had at least some expression of pSmad2 with an average $\mathrm{H}$-score of $6.06(\mathrm{SD}=3.65)$. There was no correlation between $\mathrm{pSmad} 2$ expression and ER, PR, or HER2 status. When comparing the subset of available breast primaries $(\mathrm{N}=20)$ with the matched brain metastases, there was a significantly higher mean expression level of $\mathrm{pSmad} 2$ in the brain lesion (mean $\mathrm{H}$-score $=6.75, \mathrm{SD}=3.43$ ) than in the original primary $\mathrm{BC}$ ( mean $\mathrm{H}$-score $=3.75, \mathrm{SD}=2.59)(\mathrm{p}=0.0068)$. On an individual level, higher pSmad2 expression was seen in 13 of the 20 brain lesions as compared with the original breast primary, with an average change in $\mathrm{H}$-score of $3.00(\mathrm{SD}=4.19)$. Comparison of pSmad2 staining

\begin{tabular}{|c|c|c|c|}
\hline Case & Breast H-score & Brain H-score & Difference \\
\hline 1 & 1 & 1 & 0 \\
\hline 2 & 6 & 9 & 3 \\
\hline 3 & 6 & 4 & -2 \\
\hline 4 & 1 & 6 & 5 \\
\hline 5 & 0 & 8 & 8 \\
\hline 6 & 0 & 4 & 4 \\
\hline 7 & 2 & 6 & 4 \\
\hline 8 & 6 & 6 & 0 \\
\hline 9 & 6 & 9 & 3 \\
\hline 10 & 1 & 9 & 8 \\
\hline 11 & 9 & 9 & 0 \\
\hline 12 & 4 & 6 & 2 \\
\hline 13 & 4 & 12 & 8 \\
\hline 14 & 4 & 4 & 0 \\
\hline 15 & 6 & 12 & 6 \\
\hline 16 & 4 & 9 & 5 \\
\hline 17 & 6 & 2 & -4 \\
\hline 18 & 6 & 1 & -5 \\
\hline 19 & 2 & 6 & 4 \\
\hline 20 & 1 & 12 & 11 \\
\hline Mean (SD) & $3.75(2.59)$ & $6.75(3.43)$ & $3.00(4.19)$ \\
\hline
\end{tabular}

Conclusions: $\mathrm{pSmad} 2$ is expressed in most brain metastases from $\mathrm{BC}$. When comparing the matched brain lesions to the original primary BCs, there was a significantly higher mean expression level of $\mathrm{pSmad} 2$ in the brain metastases. These findings support the hypothesis that TGF- $\beta$ and downstream signaling via Smad 2 phosphorylation are important for the development of brain metastasis from BC.

193 Upgrade to Invasive Carcinoma or Ductal Carcinoma In Situ (DCIS) in Patients Diagnosed with Lobular Neoplasia (LN) on Core Needle Biopsy (CNB): A Prospective Study

L Gilmore, S Schnitt, F Nakhlis. Beth Israel Deaconess Medical Center, Boston, MA; Brigham and Women's Hospital, Boston, MA.

Background: A wide range of upgrade rates to invasive cancer and/or DCIS has been reported for patients (pts) diagnosed with $\mathrm{LN}$ on $\mathrm{CNB}$ who undergo subsequent surgical excision. Virtually all previous studies have been retrospective and most have had few patients. Further, in prior studies not all patients with LN on CNB underwent surgical excision raising concern that in such studies selection bias influenced the reported upgrade rate. We, therefore, conducted a prospective, multi-institutional study to determine the upgrade rate at surgical excision for LN on CNB and to identify clinical, radiologic or histopathologic features that might predict upgrade.

Design: Between 2004 and 2012, following informed consent 78 patients with LN diagnosed on CNB from 5 institutions in the Translational Breast Cancer Research Consortium were prospectively enrolled in a registry to assess the frequency of upgrade to invasive breast cancer or DCIS on surgical excision following CNB. Slides of the CNBs and surgical excisions were centrally reviewed.

Results: Mean patient age was 54.5 years (range, 27-82 years). Of the 78 consented patients, 77 underwent surgical excision; one could not undergo excision because of co-morbidity. Of these 77 patients, CNB showed ALH in 48 and LCIS in 29. CNBs were most commonly performed for mammographic microcalcifications (54 pts) or density (19 pts). Other indications for CNB were MRI enhancement (2 pts) and architectural distortion (1 pt). Information on CNB indication was not available for $1 \mathrm{pt}$. The imaging abnormalities were categorized as BIRADS 4 in all cases, and following CNB all patients had concordant radiologic-pathologic findings. Of the 77 patients with $\mathrm{LN}$ on CNB who underwent surgical excision there were two upgrades $(2.6 \%)$ : one upgrade to tubular carcinoma and one upgrade to DCIS. No clinical, radiologic or histologic features were associated with upgrade.

Conclusions: In this prospective, multi-institutional study of surgical excision following a diagnosis of LN on CNB which, to our knowledge, is the first of its kind, the upgrade rate to invasive carcinoma or DCIS was $2.6 \%$. These results suggest that observation may be a reasonable alternative to surgical excision for patients with $\mathrm{LN}$ found on CNB of BIRADS 4 lesions in which there is a radiographic-pathologic concordance. Given the very low upgrade rate, we were unable to identify any clinical, radiologic or histopathologic predictors of upgrade on surgical excision.

\section{SF3B1 and SF3B3 Expression in Tamoxifen Resistance}

Y Gokmen-Polar, Y Nellamraju, CP Goswami, X Gu, SC Janga, S Badve. Indiana University, Indiananpolis, IN; IUPUI, Indianapolis, IN; Indiana University, Indianapolis, IN

Background: De novo or acquired resistance to endocrine therapy limits its usefulness in a significant number of estrogen receptor $(\mathrm{ER})^{+}$breast cancers. It is crucial to identify pathways of resistance that could provide novel targets for therapeutic intervention and improve the success of endocrine therapies. Although Oncotype Dx recurrence score (RS) predicts the likelihood of distant recurrence in tamoxifen-treated patients with $\mathrm{ER}^{+}$ breast cancer, it does not provide the mechanistic basis for endocrine resistance. Splicing Factor 3b, subunit $1(S F 3 B 1)$ mutations are described in luminal breast cancer. In this study, we evaluated the expression levels of $S F 3 B 1$ and $S F 3 B 3$ belonging to the SF3b complex, a subcomplex of the U2 small nuclear ribonucleoprotein in the spliceosome, in $\mathrm{ER}^{+}$tamoxifen resistance.

Design: Publicly available gene expression datasets were analyzed for the overexpression of $S F 3 B 1$ and $S F 3 B 3$ in breast cancer. To validate the in silico findings for the $S F 3 B 1$ and $S F 3 B 3$ expression, we further performed quantitative real-time RT-PCR (qRT-PCR) in a cohort of 60 paraffin-embedded $\mathrm{ER}^{+}$node ${ }^{-}$breast carcinomas with low, intermediate, and high Oncotype Dx RS (19, 21, and 20 cases, respectively). To further determine the correlation between endocrine resistance and $S F 3 B 1 / S F 3 B 3$, we evaluated their expression levels in an established in vitro model of $\mathrm{ER}^{+} /$tamoxifen-resistance (MCF7/ LCC2: an acquired tamoxifen resistant model of human breast cancer).

Results: In publicly available breast cancer gene expression datasets, we have identified that overexpression of the splicing factors 3 subunit 3 (SF3B3) but not SF3B1, was significantly correlated with relapse in ER+ breast cancers. qRT-PCR analysis further revealed that $S F 3 B 3$ expression is significantly upregulated in Oncotype Dx high risk groups compared to low risk $(P<0.05)$, whereas SF3B1 was not significant. In vitro, $S F 3 B 1$ and $S F 3 B 3$ levels were increased 4.02-fold $(P=0.0005)$ and 3.11 -fold $(P=0.0001)$ in $\mathrm{ER}^{+}$cells with acquired tamoxifen resistance (MCF7/LCC2), respectively. Furthermore, both $S F 3 B 1$ and $S F 3 B 3$ levels did not change in response to E2 alone or $\mathrm{E} 2$ and tamoxifen treatment in combination.

Conclusions: These findings suggest a role for the elevated expression of SF3B3 in tamoxifen resistance and recurrence of $\mathrm{ER}^{+}$breast cancer. Further studies are ongoing to determine their mechanistic role in $\mathrm{ER}^{+}$cancer and methods of targeting $S F 3 B 3$.

195

HOTAIR Expression in Breast Cancer and Nodal Metastases

$Y$ Gokmen-Polar, IT Valdislav, U Sirimalle, S Badve. Indiana University School of Medicine, Indianapolis, IN

Background: Recent studies have shown that the idea of DNA transcription resulting in synthesis of corresponding protein is rather simplistic; the process being modified by microRNAs and long noncoding RNAs (lncRNAs). At least 8000 lncRNAs have been identified. HOTAIR, (HOX Antisense Intergenic RNA), a large noncoding RNA which is 2158-nucleotides long, was described as a metastases-associated lncRNA. Its expression has been linked to increased breast, liver and colon cancer cell invasiveness. More importantly, expression was a significant predictor of metastases and death in a series of 132 breast cancer patients with extensive follow-up. Given the purported importance of HOTAIR in breast cancer, we sought to address the question whether the expression of HOTAIR could be used as a surrogate for assessing nodal metastases. Design: After appropriate IRB approval, a tissue microarray was constructed from 133 consecutive patients with breast cancer. The analysis of HOTAIR was performed using in situ hybridization methods using probes and kits obtained from Advanced Cell Diagnostic Inc (Hayward, CA). The number of signals was counted in 100 tumor cells and categorized in to four categories for statistical analysis. These categories were $0=$ less than 1 signal per 100 cells, $1=1-10$ signals, $2=10-100$ signals, and $3=$ multiple signals in each cell. None of the patients had received neoadjuvant chemotherapy. The expression of the HOTAIR IncRNA was primarily correlated with nodal status. The secondary endpoints included correlations with other clinicopathological parameters such as age, tumor size, and grade.

Results: The expression of HOTAIR could be analyzed in 94 of the 133 cases. The distribution of the scores was $0=17 \%, 1=32.9 \%, 2=30.8 \%$, and $3=19.1 \%$. The expression of HOTAIR did not correlate with nodal metastasis regardless of the scoring intensity. It also did not correlate with other parameters such as age (categorical $\leq 50$ versus $>50$ ) and tumor grade. However, it did correlate with tumor size $(P=0.02)$; more specifically, smaller tumors were more likely to be positive for HOTAIR.

Conclusions: Overexpression of HOTAIR was not associated with nodal metastases, suggesting that it might be an indicator for hematogenous metastases rather than lymphatic metastases. The correlation with small size is intriguing and needs further investigation. 
196

Upgrade Rate for High Risk Lesions Diagnosed by MRI Guided Needle Core Biopsy

KM Golden, T Thurow, M Goldberg, K Su, D Gupta, ME Sullivan. Northwestern University, Chicago, IL.

Background: The use of MRI for both screening and determining extent of disease is causing an increasing number of high risk (HR) lesions to be diagnosed on MRI guided core biopsy (CB). However, much of the literature about rates of upgrade at excision is based on traditional imaging modalities. In this study, we reviewed HR breast lesions diagnosed on MRI CB to determine if the upgrade rate is similar and if extent of pathologic changes influences the final upgrade rate.

Design: After IRB approval, MRI guided CB performed between January 2008 and June 2013 with a high risk pathology result were identified. NCB in patients with an ipsilateral BC were excluded. Lesions categorized as suspicious on the MRI and not identified on other imaging modalities were sampled using MRI guidance with a $9 \mathrm{~g}$ vacuum assisted breast biopsy. High risk lesions included atypical ductal hyperplasia (ADH), flat epithelial atypical (FEA), lobular neoplasia (LN), papillomas (IDP), and complex sclerosing lesions (CSL). All CB slides were reviewed and the extent of the HR lesions was estimated by number of cores involved and greatest extent in a single core. The excisional pathology results were obtained to determine the upgrade rate. Results: 169 CB were reviewed.

\begin{tabular}{|l|l||l||l|l||}
\hline & NMLE & Mass/Focus & Excised & Upgrades \\
\hline ADH $(\mathrm{N}=14)$ & 6 & 8 & $11 / 14(79 \%)$ & $2 / 11(18 \%)$ \\
\hline FEA (N=13) & 7 & 6 & $10 / 13(76 \%)$ & $3 / 10(30 \%)$ \\
\hline LN (N=47) & 29 & 18 & $24 / 47(51 \%)$ & $2 / 24(8 \%)$ \\
\hline CSL/IDP (N=67) & 17 & 50 & $49 / 67(73 \%)$ & 0 \\
\hline Multiple (N=28) & 9 & 19 & $25 / 28(89 \%)$ & $2 / 25(8 \%)$ \\
\hline
\end{tabular}

42 MRIs were performed for screening and the rest for extent of disease. CSL/IDP most commonly presented as a mass/focus $(75 \%)$ and showed no upgrades. Patients with multiple HR lesions had the highest rate of excision but only $8 \%$ were upgraded. LN and CSL/IDP tended to involve multiple cores ( $70 \%$ and $60 \%$, respectively). $33 \%$ of excised lesions involved only 1 core vs. $48 \%$ with no excision. Extent did not impact upgrade rate as $50 \%$ of upgraded cases only involved 1 core. ADH associated with NME had the highest upgrade rate $(50 \%)$. All upgrades were to DCIS except for one case with a $0.2 \mathrm{~cm}$ invasive lobular carcinoma.

Conclusions: In our patient population, the rate of upgrade in MRI CB is similar to more traditional imaging modalities with the exception of CSL/IDP which showed no upgrades despite often involving multiple cores. As half of the upgraded cases involved only $1 \mathrm{CB}$, extent did not predict risk of malignancy. ADH associated with NMLE had an especially high upgrade rate at $50 \%$.

197 High Density of Metastatic Intravasation Sites (TMEM) Correlate with Stem Cell Rich Tumor Microenvironments

$S$ Goswami, E Adler, E Grunblatt, JG Jones, M Oktay. Montefiore Medical Center, Bronx, NY; Yeshiva University, New York, NY.

Background: Chemotherapy targets proliferative tumor cells but misses two low proliferative populations; highly motile cells and cancer stem cells (CSCs). Patients with high proportions of these cells are poor treatment candidates and are at risk for developing metastases. To differentiate chemotherapy responders from non-responders, a method of identifying tumors with high proportions of treatment resistant cells is needed. Using immunohistochemistry (IHC) we previously identified the microanatomical sites of breast cancer cell intravasation, named TMEM. TMEM density positively correlates with distant metastases and the proportion of migratory cells, which are CSC enriched. We now questioned if TMEM rich microenvironments are also enriched for CSCs.

Design: Cells were collected from 47 human breast invasive ductal carcinomas by fine needle aspiration (FNA) of surgical resections and the percentage of $\mathrm{CD}^{+} 4^{+} / 24^{-}$ CSCs were identified using flow cytometry. TMEM density in histologic sections of the corresponding resections were evaluated with IHC using a triple stain for Mena expressing cancer cells, macrophages and vascular endothelial cells.

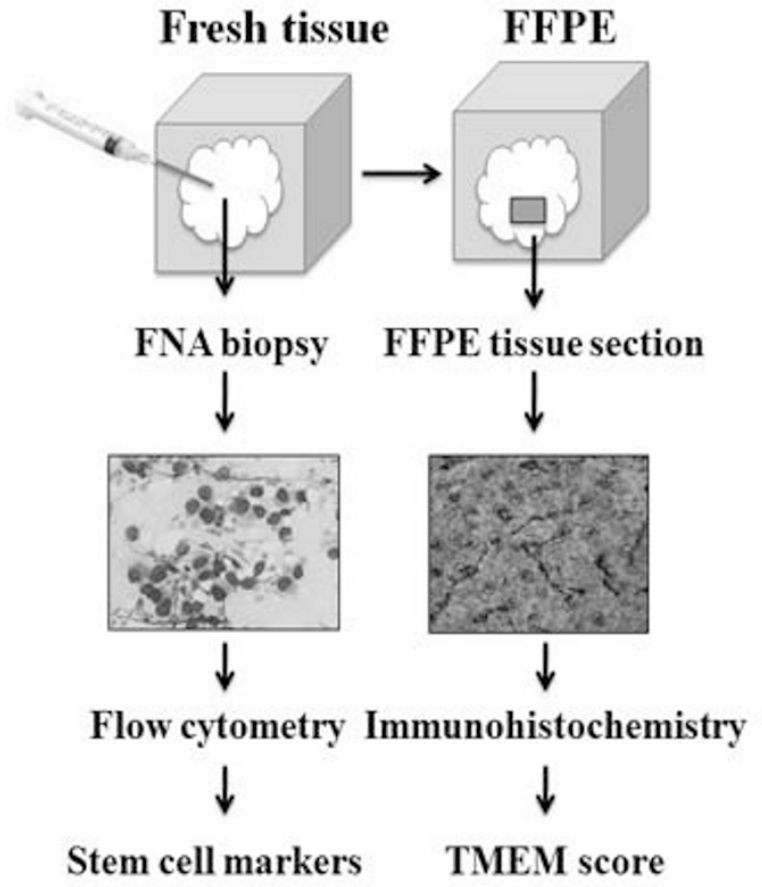

Results: TMEM density positively correlated with the percentage of CSCs across all clinical subtypes $(\mathrm{r}=.92, \mathrm{p}<.001)$.

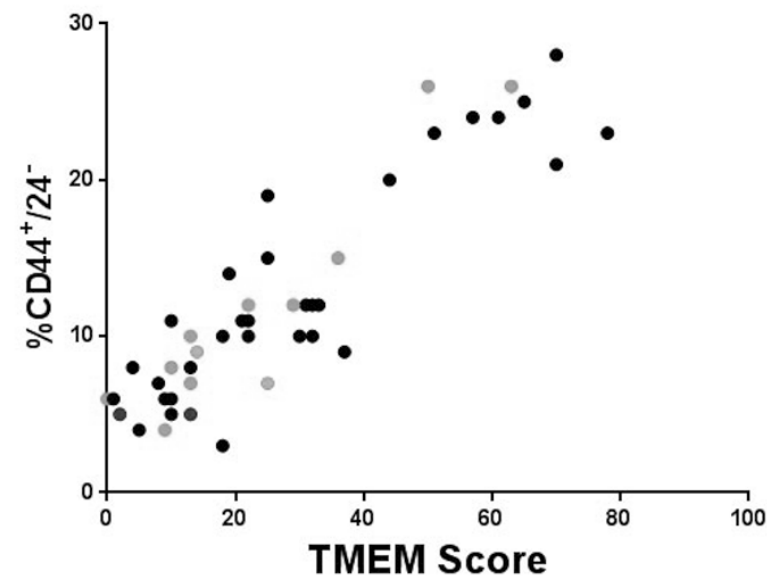

There was a significant difference in tumor size between cases with high percentage of CSC and high TMEM density $(\mathrm{P}<.001)$. The percentage of CSC and TMEM density did not correlate with the degree of differentiation, mitotic index or lymph node status. Conclusions: Microenvironments associated with metastasis in human breast cancer are enriched for CSCs. The proportion of CSCs can be successfully assessed using flow cytometry on FNA collected breast cancer cells. Additional studies are under way to determine if this approach is useful in predicting chemotherapy response.

198

Institutional Quality Assurance for Breast Cancer HER2 Immunohistochemical Testing: Identification of Outlier Practice Patterns IF Green, DL Zynger. The Ohio State University Medical Center, Columbus, OH. Background: No prior studies have compared HER2 immunohistochemical (IHC) results between pathologists within a single institution in routine clinical practice. Additionally, the impact on reporting if both HER2 IHC and HER2 fluorescent in situ hybridization (FISH) are performed in every specimen has not been evaluated. In this investigation, we analyzed HER2 IHC practice patterns among pathologists at our institution.

Design: We retrospectively retrieved pathology reports from consecutive breast needle biopsies with breast carcinoma between Jan. 2008 and Sep. 2012 at a tertiary care academic medical center with subspecialty pathology sign-out. HER2 IHC and HER2 FISH were ordered simultaneously. IHC is available 2-4 days before FISH, which is interpreted by a separate molecular pathologist. The following were recorded: IHC result and sign-out date, FISH result and sign-out date, and breast pathologist. IHC 
slides were reviewed in a blinded fashion. Pearson's $\mathrm{X}^{2}$ test, Wilcoxon rank-sum test, Cohen's kappa coefficient ( $\mathrm{k}$ ), and Chi-square goodness of fit were performed using STATA version 10 statistical analysis software.

Results: 928 cases were signed-out by 9 pathologists. HER 2 IHC results were: $67.8 \%$ negative, $11.2 \%$ positive, and $21.0 \%$ equivocal. Rates of negative (49.8-83.2\%), positive (8.7-14.1\%), and equivocal (5.2-41.5\%) varied between pathologists. There was a significant association between case pathologist and IHC score ( $<<0.0001)$, but not case pathologist and FISH score $(84.5 \%$ not amplified, $14.5 \%$ amplified, and $0.99 \%$ equivocal) $(\mathrm{p}=0.69)$. For 1 pathologist, HER2 IHC results signed-out simultaneously with FISH were significantly different than IHC results that were reported first, with an equivocal rate nearly 2 -fold lower $(10.5 \%$ vs. $20.9 \%)(\mathrm{p}=0.04)$. HER2 IHC and FISH concordance was $98.9 \%(\mathrm{k}=0.96)$. On review of HER2 IHC originally reported as IHC equivocal and FISH negative, concordance was $55.8 \%$. All discordant cases were scored as negative on review. Concordance was significantly lower for 1 pathologist $(\mathrm{p}<0.0001)$. Conclusions: Institutions should be aware that while overall HER2 IHC reporting may be consistent with established guidelines, there may be significant variation among individual practitioners. Furthermore, groups that routinely order both IHC and FISH for all cases of breast carcinoma should be aware that the availability of both test results at case sign-out may impact interpretation.

199 MicroRNA-9 Is Associated with Epithelial-Mesenchymal Transition, Breast Cancer Stem Cell Phenotype, and Tumor Progression in Breast Cancer

JM Gwak, HJ Kim, EJ Kim, YR Chung, S Yun, AN Seo, HJ Lee, SY Park. Seoul National University College of Medicine, Seoul, Korea; Seoul National University Bundang Hospital, seongnam, Korea; Asan Medical Center, Seoul, Korea.

Background: MicroRNAs (miRNAs) are involved in the progression of breast cancer. Some miRNAs, especially the miR-200 family, miR- 9 and miR- 155 have been reported to be associated with epithelial-mesenchymal transition (EMT) and breast cancer stem cell (BCSC) phenotypes.

Design: This study was designed to evaluate the expression levels of these miRNAs in human breast cancer specimens and correlate them with clinicopathologic features of the tumor including breast cancer subtype, EMT, BCSC phenotype, and prognosis. One hundred and twenty-nine patients with invasive breast cancer were included in the study. Expression levels of the miR-200 family (miR-200a, 200b, 200c, 141 and 429), miR-9, and miR-155 were quantified using qRT-PCR. Breast cancer subtype, BCSC phenotype (CD44+/CD24-, and ALDH1+), and expression of EMT markers (vimentin expression and E-cadherin loss) were evaluated by immunohistochemistry using tissue microarrays.

Results: Expression levels of the miR-200 family (miR-200a, 200b, 200c, 141 and 429), miR-9, and miR-155 were quantified using qRT-PCR. Breast cancer subtype, BCSC phenotype (CD44+/CD24-, and ALDH1+), and expression of EMT markers (vimentin expression and E-cadherin loss) were evaluated by immunohistochemistry using tissue microarrays. miR-9 was highly expressed in HER2+ and triple-negative subtypes, and was associated with aggressive features of tumor, such as high T stage, high histologic grade, $\mathrm{p} 53$ overexpression and high proliferation index. Its expression also showed correlation with the CD44+/CD24- phenotype, vimentin expression, and E-cadherin loss. Furthermore, high level of miR-9 expression was found to be an independent prognostic factor for poor disease-free survival of the patients. Expression of miR-200a and miR-141 was highest in luminal A subtype, and miR-155 expression was highest in triple-negative subtype. Although some of the miR-200 family members and miR-155 correlated with EMT or BCSC phenotype, they were not associated with patients' prognosis.

Conclusions: In conclusion, overexpression of miR-9 is associated with aggressive phenotypes and poor prognosis in breast cancer, suggesting that it can serve as a potential biomarker for breast cancer progression and a target for treatment.

200 Tumor Infiltrating Lymphocytes Are Correlated with RCBI and Ki67 in Post Neoadjuvant Breast Cancer

SW Hakim, NChang, MH Clemons, A Arnaout, DH Gravel, SJ Robertson. University of Ottawa, Ottawa, ON, Canada; The Ottawa Hospital Cancer Centre, Ottawa, ON, Canada. Background: Neoadjuvant chemotherapy (NAC) in locally advanced breast cancers (LABC) has become a mainstay modality in treatment strategies. Tumor infiltrating lymphocytes (TIL) have been associated to the sensitivity and success of NAC. Also, TIL, albeit controversial, have been correlated with complete pathologic response (pCR) and associated with various predictive factors. Correlation of Ki67 and residual cancer burden index (RCBI) to overall survival are reported in the literature. Until now, TIL have not been correlated with RCBI or Ki67 post NAC. We explore the influence of TIL on these 2 variables.

Design: We reviewed 51 patients over a period of 3 years with $\mathrm{LABC}$ who received NAC. ER/PR/HER2 and nuclear grade from the pre-NAC biopsies and post-NAC resections as well as the RCBI and Ki67 scores were reviewed. The TIL were scored by a 4 tier grading system; 0 (absent), 1 (weak), 2 (moderate), and 3 (dense). Each case was scored in pre-NAC biopsies and post-NAC resections. A separate score (dTIL) represented the difference of the two. A Pearson correlation coefficient (r) was then computed to assess the relationship between these TIL scores and each variable mentioned above.

Results: Of the 51 patients (mean age $=49.7, \mathrm{sd}=9.2), 5$ had pCR. The remaining 46 patients had RCBI scores and post-NAC ki67 indices. Pre-NAC TIL scores were: 11 $=$ score $3,24=$ score $2,13=$ score 1 and $3=$ score 0 . Post-NAC TIL scores were: $9=$ score $3,20=$ score $2,13=$ score $1,9=$ score 0 . The pre TIL score negatively correlated with the RCBI score $(r=-0.266, p=0.03)$, indicating that higher pre-TIL is associated with lower tumor burden on resection. Post TIL score showed direct correlation with the RCBI score $(\mathrm{r}=0.25, \mathrm{p}=0.03)$ and with the post resection Ki67 $(\mathrm{r}=0.41, \mathrm{p}=0.002)$, demonstrating that high levels of post-TIL were associated with an increased tumor burden/proliferation rate. The dTIL correlated with RCB $(\mathrm{r}=0.31, \mathrm{p}=0.01)$ and post nuclear grade $(\mathrm{r}=0.31, \mathrm{p}=0.01)$. HER2 showed a negative correlation both pre $(\mathrm{r}=$ $-0.41, \mathrm{p}=0.002)$ and post $(\mathrm{r}=-0.38, \mathrm{p}=0.005)$ NAC

Conclusions: These results suggest that TIL post NAC correlates with post NAC Ki67 and RCBI and therefore may be used as independent predictors for therapeutic efficacy. Although this study was limited by its retrospective design, if validated prospectively, it could be incorporated into clinical patient care. Consequently, further studies to assess TIL during active NAC, when modifications can be made, are also likely warranted.

201 Anaplastic Lymphoma Kinase (ALK) Gene Aberrations in Breast Cancers

MG Hanna, V Najfeld, J Tripodi, IY Hanna, A Nayak. The Mount Sinai Hospital, New York, NY.

Background: $A L K$, a transmembrane receptor tyrosine kinase (TK), has emerged as a novel tumorigenic factor in several cancers. Crizotinib, an $A L K$ TK inhibitor, a new FDA approved drug, is currently being used to treat lung cancer patients with EML4-ALK gene rearrangements. Recent preclinical studies have shown $A L K$ aberrations in breast cancer, particularly inflammatory breast cancer (IBC). Identifying $A L K$ aberrations in a subset of breast cancers could imply future treatment with Crizotinib. The purpose of this study was to determine the incidence of $A L K$ aberrations in relation to different breast cancer subtypes.

Design: Tissue microarrays were constructed from 133 invasive breast cancers including 13 cases of IBC, 56 ER-/PR-/HER2-, 32 ER+/HER2-, 12 ER-/HER2+, and 20 ER+/ PR+/HER2+ cases. FISH studies were performed using $A L K$ and CEP2 probes. 100 tumor cells/case were evaluated. Low copy number gain (LCNG) was defined as 3-6 fusion signals/cell, and high copy number gain (HCNG) with $>6$ signals in $>6 \%$ of cells (lab reference for $A L K$ ). Rearrangement was defined if at least 1 set of orange and green signals were $\geq 2$ signals apart in $\geq 50 \%$ of cells. IHC for $A L K$ protein expression was performed on the IBC cases.

Results: LCNG was identified in 11 IBC ( $85 \%$, range: $9-69 \%$ of tumor cells), 6 HER2+ (50\%, range: $11-68 \%$ of tumor cells), 5 ER+/HER2- $(16 \%$, range: $2-30 \%$ of tumor cells), 4 triple negative ( $7 \%$, range: $1-68 \%$ of tumor cells), and none $(0 \%)$ of the triple positive cases. $\mathrm{HCNG}$ were identified in $3 \mathrm{IBC}(23 \%$, range: $6-13 \%$ of tumor cells) and 1 HER $2+$ case $(8 \% ; 8 \%$ of tumor cells). The CEP2 analysis in HCNG cases revealed polysomy of chromosome 2 in $4 / 4(100 \%)$ cases with 3 to 6 signals noted in $51-64 \%$ of tumor cells. None of the cases showed $A L K$ rearrangement. IHC for $A L K$ protein expression was negative in all the IBC cases.

Conclusions: Neither $A L K$ gene rearrangements nor amplification was identified in the 133 breast cancer cases evaluated. We observed copy number gains (CNG) in all IBCs and a significant percentage of other breast cancer subtypes, except for the triple positive group. The results obtained with the CEP2 probe demonstrate that $\mathrm{CNG}$ are likely due to polysomy rather than amplification. The clinical significance of the role of $A L K C N G$ is yet to be elucidated. Although the lack of $A L K$ protein expression by IHC suggests no functional significance of $\mathrm{CNG}$, larger studies are required to investigate the role of $A L K$ copy number alterations in breast cancer. This study is one of the largest studies to have investigated $A L K$ gene aberrations in breast cancer and the only study to include all subtypes.

202 Prognostic Value of Coexisting Lobular Carcinoma In Situ with Invasive Lobular Carcinoma

A Harbhajanka, I Lamzabi, S Syed, J Macagba Slade, P Gattuso. Rush University, Chicago, IL.

Background: Recent studies show that Lobular carcinoma in situ (LCIS) and invasive lobular carcinoma (ILC) share a similar genetic molecular biology. With this new emerging evidence, there are increasing concerns regarding the biological significance of LCIS. Aim of this study is to clarify whether the presence of coexisting LCIS in ILC affects tumor biology and behavior to correlate it with other clinicopathologic parameters.

Design: 254 cases of ILC were included and patient with ILC and coexisting LCIS(ILC+LCIS) were compared with pure ILC cases with respect to clinicopathologic parameters. Immunohistochemical (IHC) stains for ER, PR, E-cadherin, HER2, MIB-1 and FISH for HER2 of 254 ILC cases were retrieved.

Results: Of the 254 cases, 107 cases were pure ILC and 147 cases were ILC+LCIS. Seventy seven $(41.62 \%)$ cases showed axillary lymph node metastases. Nodal involvement, recurrence and metastasis of ILC with LCIS is less frequent compared to ILC without LCIS with p-value of 0.034 and 0.007 respectively. The presence of coexisting LCIS in ILC predicts higher disease free survival compared to pure ILC $(\mathrm{p}=0.034, \log$ rank $)$. 


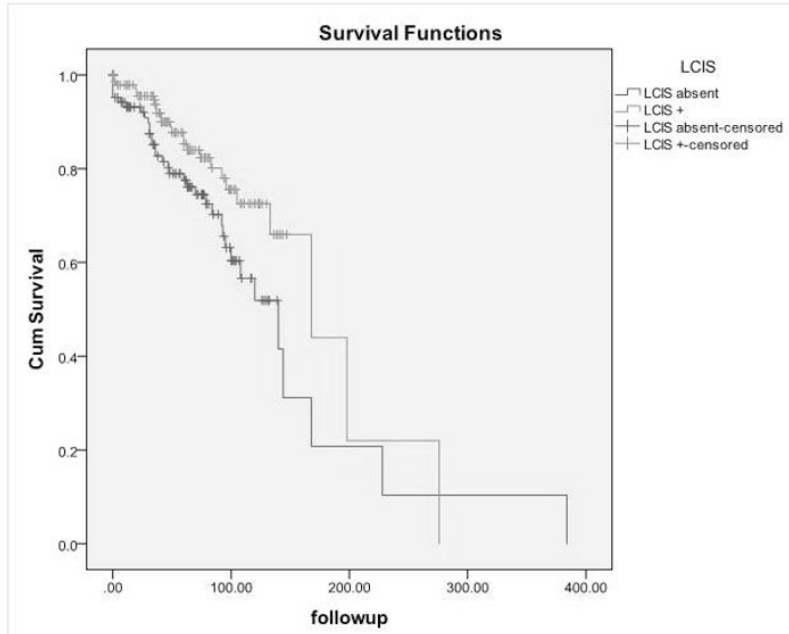

When divided into different strata, ER positive ILC cases with associated LCIS cases show better disease free survival (DFS) then ER positive pure ILC ( $\mathrm{p}=0.021, \log$ rank).
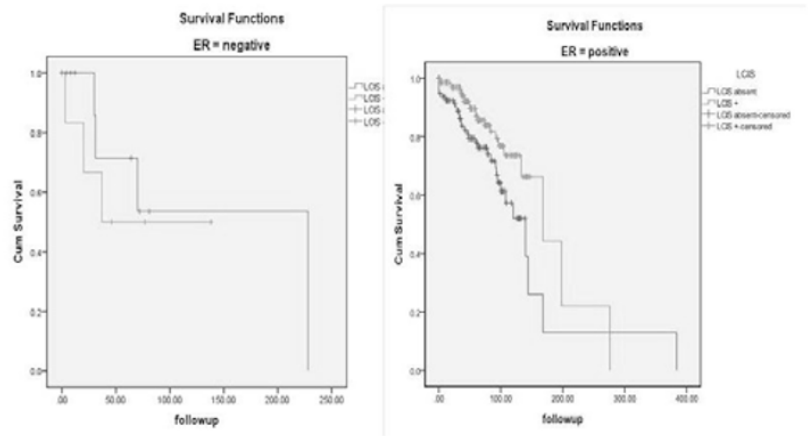

Similarly, ILC cases with LCIS in patient $<50$ years show better DFS then the patient $<50$ years with pure ILC ( $\mathrm{p}=0.045, \log$ rank).

Conclusions: In conclusion, invasive lobular carcinoma coexisting with lobular carcinoma in situ is characterized by less nodal involvement, lower recurrence and metastatic propensity and better disease free survival than pure ILC. When divided into different strata, ER positive and $<50$ years groups with ILC + LCIS show even significant better DFS then pure ILC. These findings suggest that there is biological significance of coexisting LCIS in ILC and even have more effects on tumor aggressiveness in certain strata of ILC.

\section{Prophylactic Mastectomies: Trends over More Than 20 Years at a Single Institution}

BT Harrison, A Lo, B Singh. New York University, New York, NY; Southern California Permanente Medical Group, Fontana, CA.

Background: Recent studies indicate that the number of prophylactic mastectomies has increased over the last decade. We aimed to determine the trends in number of prophylactic mastectomies performed at our institution over more than 20-years, the prevalence of pathology within these specimen, and whether the implementation of a more comprehensive grossing protocol influenced the rate of detection of high-risk lesions.

Design: All prophylactic mastectomies, including both contralateral prophylactic mastectomies (CPM) in patients with ipsilateral breast cancer and bilateral prophylactic mastectomies (BPM) in patients with genetic or familial predisposition, were retrospectively identified from the departmental database from January 1992 to July 2013. From 2006 onward, prophylactic mastectomies were grossed according to a protocol requiring four random sections from each quadrant in addition to sections from the nipple and subareolar tissue. Reduction mammoplasties identified from this same time period served as controls. Statistical analysis employed Fisher's exact test. Results: In total, 601 CPM, 129 BPM and 1302 reduction mammoplasties were performed. Specimen from women with BRCA mutations accounted for $9.3 \%$ of CPMs and $47.3 \%$ of BPMs. CPMs have steadily risen over the years with averages of 4.6 , 11, 23.2, and 67.2 per year over successive 5-year intervals from 1992 to 2011. BPMs significantly increased from an average of 0.64 per year from 1992 to 2005 to 18.7 per year from 2006 to 2011, and significantly decreased to an average of 3 per year since then. DCIS or invasive carcinoma was found in 37 cases $(6.2 \%)$ of CPMs and 6 cases $(4.7 \%)$ of BPMs and ADH, ALH and LCIS in 148 cases $(24.6 \%)$ of CPMs and 21 cases $(16.3 \%)$ of BPMs. Most pathologic findings were significantly increased in prophylactic mastectomies versus controls. There was no statistical difference in the detection of pathologic findings before and after changes to grossing protocols in 2006. Conclusions: To our knowledge, this is the largest single-institution retrospective study of pathologic findings in prophylactic mastectomy specimen to date. The number of prophylactic mastectomies has significantly increased in recent years. Four random sections from each quadrant provide adequate sampling from prophylactic mastectomy specimens. Rates of occult malignancy (DCIS/IC) and high-risk lesions (ADH, ALH, LCIS) are comparable to those reported in other large studies of CPMs (4-6\% and $15-28 \%$, respectively) and lower than those reported for BPMs (13-15\% and $33-42 \%$, respectively).

204 Peri-Angiolymphatic Tumor-Infiltrating Leukocytes May Predict Angiolymphatic Invasion and Metastatic Lymph Node Status in Breast Carcinoma

JM Hawkins, SM Cavone, RC Heintzelman, MD Zarella, FU Garcia. Drexel University College of Medicine, Philadelphia, PA.

Background: Peri-angiolymphatic leukocytes (PALs) are a subset of tumor infiltrating lymphocytes that are associated with tumor growth, angiogenesis, invasiveness and metastatic potential. We studied the leukocyte infiltration in the peri-angiolymphatic space in areas at the periphery of the tumor to determine if the number of leukocytes and the subtype can be correlated with angiolymphatic invasion (ALI) and lymph node metastasis.

Design: Thirty-five cases with primary invasive mammary carcinoma treated with definitive surgery were retrieved from an IRB approved database. Twenty-three consecutive $\mathrm{pN} 1$ and twelve consecutive pN0 cases were included in this study. All the H\&E slides were reviewed, an the slide from each case with the most PALs was selected for immunohistochemistry and for identification of angiolymphatic invasion. CD3, CD4, CD8, and CD68 antibodies were utilized to characterize the leukocytes. The angiolymphatic spaces with surrounding inflammation were further classified with CD31 and D2-40 antibodies. The slides were scanned using Aperio Scanscope XT and the PALs were quantified using the Aperio cytoplasmic algorithm. The leukocyte subtypes were enumerated and statistically analyzed using ROC analysis.

Results: Patient age ranged from 33 to 80 years. CD31 and D2-40 were used to determine whether vascular or lymphatic spaces exhibit PALs. Within the pN0 subset, CD31 was expressed in $11(91.7 \%)$ cases and $1(8.3 \%)$ expressed D2-40. In the pN1 subgroup, $4(11.4 \%)$ cases expressed only CD31, $6(17.1 \%)$ cases expressed only D2-40 and 13 (37.1\%) cases had both lymphatic and vascular spaces (D2-40 and CD31 expression). ROC analysis was used to compare PALs expression between ALI and no ALI . The area under the ROC curve (aROC) was 0.71 for CD3+ lymphocytes, and 0.62 for both $\mathrm{CD} 8+$ and CD68+ leukocytes for the presence of ALI. To compare $\mathrm{pN} 0$ and $\mathrm{pN} 1$, aROC was 0.65 and 0.68 for $\mathrm{CD} 3+$ and $\mathrm{CD} 4+$ lymphocytes respectively, which suggests that PALs are predictive for both ALI and nodal status.

Conclusions: PALs in pN0 tumors surround vascular channels and in pN1 tumors surround both vascular and lymphatic spaces. PALs are more predictive for angiolymphatic invasion than for metastatic status. CD4+ cells are predictive for nodal status, while CD8+ cells are predictive for angiolymphatic invasion, which suggests early endothelial injury. The presence of PALs at the periphery of the tumor should alert the pathologist for the presence of angiolymphatic invasion.

205 Molecular Mapping of Triple Negative Invasive Ductal Carcinoma in African-American Women

$J$ He, R Zreik, E Dauway, K Walker, L Watson, P Zhang, D Jupiter, A Rao. Scott \& White Memorial Hospital, Temple, TX; Texas A\&M Health Science Center, Bryan, TX. Background: Molecular mechanisms of triple negative breast cancers (TNBC) are being increasingly explored to facilitate discovery of new therapeutic targets and understand the mechanisms behind this aggressive phenotype. TNBC has been identified as a common phenotype in African American (AA) women of all ages and is associated with a higher stage and aggressive behaviour. Though molecular mechanisms and gene expression data are being increasingly studied in TNBC, very limited data is available on $\mathrm{TNBC}$ in the AA population. This study presents gene expression in epithelial to mesenchymal transition (EMT) genes and PI3K pathway genes as also preliminary next generation sequencing data in TNBC in the AA group.

Design: A retrospective search of the Scott \& White Hospital database identified 35 African American women with triple negative breast cancer resection samples from 2008 to 2012 with follow up clinical data. Formalin-fixed, paraffin embedded, blinded tumor resections was evaluated for the epithelial to mesenchymal transition (EMT) gene expression as also gene expression of the PI3K-AKT signaling pathway components using RT ${ }^{2}$ ProfilerTM PCR Array expression kits containing 84 genes each. Tumor DNA was also analyzed by next generation sequencing (NGS) for mutations in 400 cancer hot spot gene panel using Ion Torrent PGM. Activating mutations at the 3 mutational hot spots of the PI3K gene (codons 542, 545, and 1047) were also tested by PCR using Qiagen PI3K Mutation Test Kit on the RotorGene instrument.

Results: EMT gene expression showed all tumors had the WNT pathway gene expression down regulated. Early stage (stage1) breast cancers over-express the cytokeratin 19 and TGFB in ER signaling pathway and RAC1 in PI3K pathway, whereas late stage (stage 4) cancers do not. The most impressive finding was the presence of PIK3 hot spot mutations in $79 \%$ of cases, including 10 at codon 542,11 at codon 545 , and 6 at codon 1047, confirmed by real time PCR assay. Preliminary NGS identified multiple mutations in PI3K pathway.

Conclusions: Our data suggest that distinct differences including CK 19 over-expression and PI3K mutations characterize African American triple negative breast cancers. These may serve as early distinct prognosis markers and/or as early therapeutic target in the African American population. 
Telomere Length Alterations in Invasive Lobular Carcinomas

CM Heaphy, P Argani, AK Meeker, A Cimino-Mathews. The Johns Hopkins University School of Medicine, Baltimore, MD.

Background: Invasive lobular carcinoma (ILC) is the second most common form of breast cancer and represents $10-15 \%$ of all invasive breast cancers. Telomeres, nucleoprotein complexes located at the extreme ends of eukaryotic chromosomes, protect chromosome ends from degradation and recombination. Dysfunctional telomeres contribute to genomic instability and promote tumorigenesis. Alterations in telomere length have been reported in many tumor types, including breast, and have been associated with cancer risk and prognosis. Short telomere lengths have been associated with triple negative and Her2+ ductal carcinomas. However, no previous investigations have specifically assessed ILC cases. Here, we evaluate telomere lengths within ILC cases with complete characterization of estrogen receptor (ER), progesterone receptor (PR), and Her2 status.

Design: We evaluated tissue microarrays with 34 ILC cases, containing 32 luminal A $(\mathrm{ER}+/ \mathrm{PR}+/ \mathrm{Her} 2-), 1$ luminal B (ER+/PR+/Her2+), and $1 \mathrm{Her} 2+(\mathrm{ER}-/ \mathrm{PR}-/ \mathrm{Her} 2+)$. A telomere-specific fluorescence in situ hybridization (FISH) assay that provides single cell resolution of telomere length while maintaining tissue architecture was used. Telomere lengths were qualitatively scored by direct visual assessment of the stained slides, comparing telomere signals from tumor cells to telomere signals from benign cells from the same case. Overall telomere length was compared to standard clinical and pathological markers.

Results: Out of the 34 cases, 19 (56\%) displayed either normal or long telomeres, whereas only 15 (44\%) displayed short telomeres. Both the luminal B and Her2+ cases displayed short telomeres. Telomere length was not associated with stage, grade, tumor size, age, and race. However, the cases with normal or long telomeres had an overall higher Ki-67 index compared to the cases with short telomeres $(\mathrm{p}=0.039)$. Interestingly, 3 cases $(9 \%)$ with normal or long telomeres demonstrated a unique telomere pattern consisting of a single bright telomere spot among the normal telomere signals within each individual cancer cell.

Conclusions: A smaller subset of ILC cases demonstrate short telomeres than previously studied invasive ductal carcinomas, which may reflect the smaller proportion of luminal $\mathrm{B}, \mathrm{Her} 2+$ and triple negative carcinomas in this tumor type. Telomere length may have utility as a prognostic marker in invasive lobular carcinomas. Additional studies are needed to further evaluate the significance of the unique single spot telomere phenotype described here, which has not been previously described in any tumor type.

207 Characteristics of Lymphovascular Invasion in Differen Histologic Subtypes of Invasive Breast Carcinoma

$C$ Hilliges, $M$ Gallagher, $M$ Stempel, M El-Tamer, E Brogi. Memorial Sloan-Kettering Cancer Center, New York, NY; Karolinska University Hospital, Stokholm, Sweden. Background: The presence of tumor cells within a lymphatic or vascular space (LVS) constitutes lymphovascular invasion (LVI). The relationship between LVI and histologic subtypes of invasive breast carcinoma (BC) has not been fully evaluated. We sought to characterize and quantify LVI in a cohort of patients (pts) with $\mathrm{BC}$ and correlate the findings with $\mathrm{BC}$ histologic subtype.

Design: We identified consecutive pts with unilateral T1-T2 BC, metastases to 1-3 lymph nodes (LN), and index diagnosis of LVI who underwent mastectomy at our Center. We excluded pts treated with neoadjuvant chemotherapy, with isolated tumor cells in LNs, unavailable slides or no LVI on slide re-review. A pathologist (C.H.) assessed BC type and grade, and quantified amount and characteristics of LVI near and away from BC. Another pathologist (E.B.) reviewed problematic cases. ER, PR and HER2 data were retrieved from the pathology records.

Results: Our study cohort consists of 135 female pts with a median age of 51 years (range 33-89). The histologic types of BC and the characteristics of the associated LVI are summarized in the table.

\begin{tabular}{|c|c|c|c|c|c|c|c|}
\hline Tumor type & 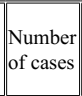 & $\begin{array}{l}\text { Tumor size } \\
(\mathrm{mm}) \text { (range) }\end{array}$ & $\begin{array}{l}\text { Mean } \\
\text { number LVI } \\
\text { foci (range) }\end{array}$ & $\begin{array}{l}\text { Mean size } \\
\text { largest tumor } \\
\text { embolus }(\mathrm{mm}) \\
\text { (range) }\end{array}$ & \begin{tabular}{|l|} 
Cases \\
with \\
occlusive \\
LVI
\end{tabular} & ER+ & HER 2+ \\
\hline Apocrine & 6 & $2.7(1.9-5)[* 3]$ & $11(1-31)$ & $0.5(0.15-0.9)$ & 4 & 2 & 3 \\
\hline $\begin{array}{l}\text { Pleomorphic } \\
\text { ILC }\end{array}$ & 5 & $2.8(2-4.5)[* 4]$ & $4(1-10)$ & $0.2(0.05-0.35)$ & 1 & $\mid 5$ & 2 \\
\hline Micropapillary & 5 & $1.5(1.0-2.1)[* 3]$ & $254(3-638)$ & $3.1(0.2-14)$ & 1 & 5 & 0 \\
\hline Neuroendocrine & 3 & $3.6(2.8-4.4)$ & $23(3-61)$ & $0.5(0.4-0.6)$ & 3 & 3 & 0 \\
\hline $\begin{array}{l}\text { Mixed ductal } \\
\text { and lobular }\end{array}$ & |2 & $2.6(2.5-2.7)[* 1]$ & $16(7-25)$ & $0.2(0.2-0.2)$ & 0 & | 2 & 0 \\
\hline Classical ILC & 1 & 4.5 & 1 & 0.4 & 1 & 1 & 0 \\
\hline IDC NOS & 113 & $2.2(0.7-5)[* 29]$ & $26(1-100)$ & $1.0(0.03-18)$ & 32 & 88 & 14 \\
\hline All cases & 135 & $2.3(0.7-5)[* 44]$ & $33(1-638)$ & $1.0(0.03-18)$ & 42 & 106 & 19 \\
\hline
\end{tabular}

[*N of multifocal cases]

Conclusions: The mean number of foci of LVI was highest in micropapillary BC, but invasive ductal carcinoma NOS and neuroendocrine BC also had more than 20 foci of LVI/case. In the patient population studied, occlusive LVI, a morphologic mimic of DCIS, was most common in neuroendocrine BC ( $3 / 3$ cases) and apocrine BC ( $4 / 6$ cases), and occurred in nearly a third of invasive ductal carcinoma NOS. Our results detail the features of LVI in different subtypes of BC with 1-3 positive LNs. This information will assist pathologists in recognizing LVI in BC, expecially when only limited tissue is available.
208 Morphologic Features and Prognostic Value of Lymphovascular Invasion in Lymph Node Positive Breast Carcinoma

$C$ Hilliges, $M$ Hsu, $M$ Gallagher, $M$ Stempel, M El-Tamer, E Brogi. Memorial SloanKettering Cancer Center, New York, NY; Karolinska University Hospital, Stockholm, Sweden.

Background: Lymphovascular invasion (LVI) indicates presence of tumor emboli (TE) within lymphatic or vascular spaces (LVS). Although the extent of LVI can vary considerably, it is usually reported only as present or absent. LVI correlates with worse outcome in patients (pts) with invasive breast carcinoma (BC) and negative lymph nodes (LN-neg), but the prognostic significance of LVI in LN-positive (LN-pos) pts is unclear. Design: We identified pts with T1-T2 BC, 1-3 LN-pos and reported LVI who underwent mastectomy at our Center between 1995 and 2006. Pts with bilateral BC, status-post neoadjuvant chemotherapy and/or post-mastectomy radiotherapy, unavailable slides or no evidence of LVI at slide re-review were excluded. A pathologist (C.H) reviewed all cases, recorded $\mathrm{BC}$ features and quantified amount and characteristics of LVI near and away from BC, including number of involved LVS and largest TE size. Problematic cases were reviewed with another pathologist (E.B.). Clinical follow-up was extracted from a service database.

Results: Ninety-eight pts, all women, met the study inclusion criteria. Pts median age at diagnosis was 51 years (range 34-89). The median tumor size was $2.1 \mathrm{~cm}$ (range 0.7-5.0) (10 T1b, $31 \mathrm{~T} 1 \mathrm{c}$ and $49 \mathrm{~T} 2 \mathrm{BC})$. A median of $17 \mathrm{LNs} / \mathrm{pt}$ were examined (range 2-44): $53 \%$ pts had $1 \mathrm{LN}-$ pos, $33 \%$ had $2 \mathrm{LN}-$ pos and $14 \%$ had $3 \mathrm{LN}-$ pos. The median number of LVS with TE/pt was 8 (range 1-317): $26 \%$ pts had $1-2$ involved LVS; $33 \%$ had $3-10$ and $42 \%$ had $>10$. The median size of the largest TE was $0.3 \mathrm{~mm}$ (range $0.03-$ 18 ) and the median occlusion ratio (=diameter largest TE/diameter of corresponding LVS) was $73 \%$ (range $2-100 \%$ ). In 28 cases (29\%) at least one TE completely filled a LVS. The median follow-up time was 73 months (range 2-152). 17 (17\%) pts developed recurrent disease that was local (1 pt), distant ( 9 pts) or both $(7 \mathrm{pts})$. Seven pts died of disease. Kaplan-Meier estimate of the 5 -year event-free rate was $86 \%(95 \% \mathrm{CI})$. Cox regression analysis found no association between LVI and outcome.

Conclusions: In pts with 1-3 LN-pos T1-T2 BC, extensive LVI is a common finding. Our results suggest that extent and morphologic characteristics of LVI do not add any further predictive value to its presence; detailed quantification of LVI in this setting seems unnecessary.

209 Expression of Tissue Inhibitors of Metalloproteinase 1 and 3 in a Spectrum of Proliferative Breast Lesions

CV Hojilla, G Kuruzar, R Khokha, MC Chang. Mount Sinai Hospital, Toronto, ON, Canada; University of Toronto, Toronto, ON, Canada; Princess Margaret Hospital and University of Toronto, Toronto, Canada.

Background: The Tissue Inhibitors of Metalloproteinases (TIMPs) are important protein mediators of stromal remodeling in both normal development and invasion by breast carcinoma. Overexpression of TIMP-1 and TIMP-3 has been observed both in more aggressive and more chemotherapy-resistant tumors. Although the TIMPs are potential breast biomarkers, TIMP expression is not well characterized in benign and premalignant breast lesions. Our aim was to describe TIMP-1 and TIMP-3 expression in a range of breast tissues.

Design: TIMP-1 (VT-7 clone) and TIMP-3 (polyclonal) immunohistochemical staining (heat retrieval in citrate, MACH4 protein polymer detection with DAB chromogen) was optimized using appropriate controls. Paraffin-embedded breast tissues (24 blocks from 10 different cases) were selected to represent normal breast and a range of proliferative breast lesions. Benign breast lesions included apocrine metaplasia, papillomas, sclerosing adenosis, complex/radial sclerosing lesions, and epithelial hyperplasia. Neoplastic lesions included ADH/ALH, DCIS, and tissues with invasive carcinoma associated with in situ disease.

Results: In normal breast, benign sclerosing lesions, and apocrine lesions, TIMP-3 was expressed in all cases, whereas TIMP-1 was negative in all cases. Both TIMP-1 and TIMP-3 were highly expressed in $80 \%$ of DCIS cases, $50 \%$ of columnar-cell and papillary lesions. Low-to-moderate TIMP-1 and TIMP-3 co-expression was seen in simple adenosis. Where DCIS was present in association with invasive carcinoma, the in situ component had strong TIMP-1 and TIMP-3 expression, whereas the invasive component did not consistently overexpress either TIMP.

Conclusions: Although TIMPs are possible predictive biomarkers for breast carcinoma, they are also highly expressed in a subset of benign lesions and in DCIS. TIMP-1 may be more closely associated than TIMP-3 with neoplastic change in glandular epithelium.

210 The Matrix Associated Protein, CCN6/WISP3, Reduces Tumor Initiating Cells in Triple Negative Breast Cancer

$W$ Huang, ME Gonzalez, CG Kleer. University of Michigan, Ann Arbor, MI.

Background: Triple negative breast carcinomas are an agressive group of tumors reported to be enriched in stem cells. The transition between epithelial and mesenchymallike states, a process which occurs in embryogenesis, is important for tumor invasion. The acquisition of mesenchymal-like features has been linked to increased self renewal and tumorigenicity. CCN6 is an extracellular protein with tumor suppressor functions in breast cancer. Our laboratory has found that CCN6 downregulation in benign breast cells induces an epithelial to mesenchymal transition and triggers invasion. We hypothesize that upregulation of CCN6 in triple negative breast cancer decreases tumorigenicity by altering the cell phenotype and the tumor initiating cell population.

Design: We overexpressed CCN6 in triple negative breast cancer cell lines, MDAMB-231 and SUM-159, which have mesenchymal-like features and low levels of endogenous CCN6 protein. We investigated the morphological changes induced by CCN6 upregulation, as well as its effect on cell motility and invasion. The role of CCN6 on stem cells was studied using mammosphere assays and the Aldehyde dehydrogenase 
(ALDH1) assay by FACS. The effect of CCN6 overexpression on the tumor initiating capacity was tested by injecting ADLH1+ and ALDH1- populations of MDA-MB-231 CCN6 overexpressing cells and controls in the mammary fat pads of NOD/SCID mice. Results: CCN6 upregulation in triple negative breast cancer cells induced a morphological change from mesenchymal-like to epithelial, and decreased breast cancer cell motility and invasion. In tandem, CCN6 upregulation increased cytokeratin 18 and E-cadherin, and decreased vimentin and the transcription factor Slug (Snail2). CCN6 reduced the number of mammospheres and of ALDH1+ cells. Reconstitution of Slug expression reversed the tested effects of CCN6 overexpression, indicating that Slug mediates CCN6 functions. In vivo, CCN6 overexpression significantly delayed tumor formation, and decreased tumor volume and metastasis in the ALDH1+ and ALDH1populations of MDA-MB-231 cells compared to controls.

Conclusions: Our data suggest a novel function of CCN6 in regulating Slug-induced epithelial to mesenchymal transition states and the tumor initiating cell population of triple negative breast cancer cells.

211 The Rate of Upgrade for Pure ADH, Pure FEA and Mixed ADH/FEA in Mammographically Detected Lesions: A Study of 249 Cases

X Huang, X Chen, D Wang, S Liu, T Khoury. Roswell Park Cancer Institute, Buffalo, NY. Background: It is established that atypical ductal hyperplasia (ADH) has higher risk of upgrade than flat epithelial atypia (FEA) diagnosed on a core needle biopsy (CNB). $\mathrm{ADH}$ and FEA frequently coexist in the same core. However, the rate of upgrade of this lesion is unclear.

Design: We reviewed 103 pure ADH cases, 46 pure FEA, and 100 mixed FEA/ADH from RPCI files between 2006 and 2013. We recorded the reason for biopsy (mass or microcalcifications). Clinical information including patient age, race, menopausal status, and history of breast cancer (concurrent or past, ipsilateral or contralateral) are also recorded. The slides were reviewed. In mixed ADH/FEA, the percentage of FEA was estimated. Upgrade is defined as presence of invasive carcinoma (IC) or ductal carcinoma in situ (DCIS). Fisher exact test was used to examine the association of the rate of upgrade between each two categories.

Results: Among the 249 cases, the upgrade rate was $24.1 \%$, with the upgrade rates for pure $\mathrm{ADH}$, pure FEA and mixed $\mathrm{ADH} / \mathrm{FEA} 30.1 \%, 6.52 \%$ and $26 \%$, respectively. Figure 1 shows the rate of upgrade with each level of FEA percentage. Both pure ADH and mixed ADH/FEA had higher rate of upgrade comparing to pure FEA ( $p=0.0031)$. The rate of upgrade of pure ADH vs. mixed ADH/FEA had no statistically significant difference. The relative risk of upgrade in mixed FEA/ADH vs. pure FEA is 3.99 with $95 \%$ CI of $[1.27,12.50]$. Based on the p-value, the optimal cutoff of FEA component in mixed $\mathrm{ADH} / \mathrm{FEA}$ of predicting upgrade is $\leq 50 \%$ with sensitivity of $78.3 \%$ and specificity of $43.4 \%(\mathrm{p}=0.002)$. Based on the area under the curve, the best cutoff is $\leq 80 \%$ with sensitivity of $91.7 \%$ and specificity of $31.8 \%(\mathrm{p}<0.001)$. When there is a history of breast cancer, pure ADH predicts DCIS (7/36) and mixed ADH/FEA predicts IC $(6 / 6)(\mathrm{p} \leq 0.001)$

Conclusions: While the rate of upgrade of FEA is significantly less than ADH, mixed FEA/ADH had similar rate of upgrade to pure ADH. The history of breast cancer could help predicting the type of upgrade IC vs. DCIS.

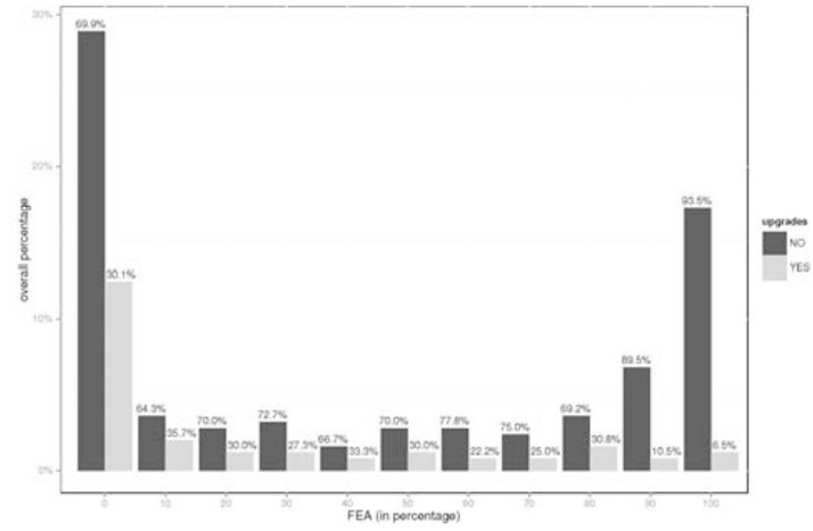

212 Electron Microscopy of Encapsulated and Solid Papillary Carcinoma, Is This an In-Situ or an Invasive Carcinoma

$J$ Hugh, S Silverman, K Chung, $R$ Vriend. Misericordia Hospital, Edmonton, AB, Canada; university of alberta, Edmonton, AB, Canada.

Background: For long time encapsulated and solid papillary carcinomas have been debated either an in-situ or an invasive entity. The goal of our study was to examine the presence or absence and the quality of myoepithelial cells, the presence or absence and thickness of basement membrane in all the selected cases.

Design: 8 cases of encapsulated and solid papillary carcinomas, 3 cases of low to intermediate grade DCIS in association with low grade IDC NOS and 2 cases of combined IG DCIS and encapsulated papillary carcinomas as well as 2 cases of normal breast were selected from your database. The morphology was reviewed, immunohistochemical stains to highlight myoepithelial cells were performed and all cases were subjected for digital electron microscopy

Results: All 5 cases of encapsulated papillary carcinoma show the presence of continuous or discontinuous attenuated basement membrane and absence of myoepithelial cells, 3 solid papillary carcinomas show possible small myoepithelial cells. Conclusions: We think that encapsulated papillary carcinomas represent a category of neoplasms in transition from an in-situ to invasive carcinomas, and at this stage should be interpreted as low grade invasive ductal carcinomas with favorable behavior. Solid papillary carcinomas should be divided into 2 categories: an invasive solid papillary carcinoma and an in-situ solid papillary carcinomas.

213 Clinico-Pathological Determinants of Neoadjuvant Treatment Response in Breast Cancer: An Analysis of $\mathbf{7 5}$ Cases

AR Husain, RY Tsang, H Yang. University of Calgary, Calgary, AB, Canada.

Background: The use of neoadjuvant chemotherapy for locally advanced and early stage breast cancer is on the rise. However, clinico-pathological factors which influence treatment response remain unclear. The purpose of this study is to investigate common clinico-pathological characteristics of treatment responders versus non-responders in an attempt to improve insight into the mechanisms of treatment response, thereby optimizing treatment strategies.

Design: Seventy five cases of breast cancer that received neoadjuvant chemotherapy at our institution between 2010-2012 were identified. An average of 6-8 cycles of neoadjuvant chemotherapy is the institutional standard, including trastuzumab in HER2 positive cases. Cases were divided into pathologic complete response (pCR), partial response (pPR) and no response (pNR) groups based on current AJCC Cancer Staging for Breast Cancer, $7^{\text {th }}$ edition. Clinico-pathological variables including age, tumor type, grade and biomarker status including ER, PR, HER2 for each group were analyzed and compared.

Results: Of the 75 neoadjuvant cases, 16 (21.3\%) patients achieved pCR ; $10(13.3 \%)$ pNR and $49(65.3 \%)$ pPR. The pCR group ranged from ages 27 to 72 years with a mean age of 48 years. All cases were invasive ductal carcinoma (IDC). Tumor grade was II in two cases and III in fourteen cases. ER/PR was positive in six and negative in ten cases. HER2 was positive in eight and negative in eight cases. In contrast, the pNR group ranged from ages 47 to 65 years with an average age of 51 years. The tumor type was IDC in 7 cases, invasive lobular carcinoma (ILC) in 2 cases and mucinous in 1 case. The tumor grades were I in two cases, II in four cases and III in four cases. The ER/PR was positive in eight cases and negative in two. HER2 was negative in seven cases and positive in three. For the pPR group, the ages ranged from 26 to 75 years with a mean age of 51 years. Thirty cases were MBR grade III, 18 grade II and 1 grade I. Her2 was negative in 34 cases and positive in 15 . ER/PR was positive in 37 cases and negative in 12 .

Conclusions: Consistent with previous reports, the $\mathrm{pCR}$ group was associated with higher grade and ER/PR negativity, with an increased tendency for HER2 positivity. In contrast, the pNR group was associated with lower grade including non-ductal types (lobular and mucinous carcinoma), and were more likely to be ER/PR positive and HER2 negative.

214 Human Equilibrative Nucleoside Transporter1 (hENT1) May Play a Role as Predictive Biomarker in Triple Negative Breast Carcinoma

J Iqbal, AA Thike, LK Hon, PH Tan. Singapore General Hospital, Singapore, Singapore. Background: Recent evidence suggests human equilibrative nucleoside transporter 1 (hENT1) is a prognostic biomarker in gemcitabine-treated pancreatic cancer, and may play a role as a predictive biomarker of gemcitabine efficacy. Interestingly, the gemcitabine/platinum combination has demonstrated activity in metastatic breast carcinoma as well as triple negative breast carcinoma (TNBC). An immunohistochemical assay may assist in determining hENT1 expression in TNBC allowing us to determine whether post-therapy outcomes can be predicted based on pretreatment hENT1 protein expression.

Design: Immunohistochemistry was performed on paraffin-embedded tumor tissue of a consecutive cohort of 114 female patients diagnosed with triple negative breast cancer. hENT1 was scored on the basis of relative staining of lymphocytes as internal positive control. Staining intensity was graded as absent (0), positive but less intense than lymphocytes $(1+)$, positive, similar intensity as lymphocytes $(2+)$, and positive, more intense than lymphocytes $(3+)$. Tumors with intensity staining $(2+$ and $3+)$ in $>75 \%$ of the tumor cells were considered as high expression of hENT1. Follow-up information was obtained from case-notes. Disease free survival (DFS) and overall specific survival (OS) was defined as time from diagnosis to recurrence or death respectively. Associations between hENT1 with clinicopathological parameters, DFS and OS were evaluated. A $\mathrm{p}$ value of $<0.05$ defined statistical significance.

Results: hENT1 was overexpressed in $29 \%$ of cases and was negative in one case. It showed statistically significant correlation with median age and tumor size. However no correlation with either grade or lymphovascular invasion was observed. Over-expression of hENT1 showed nonstatistically significant trend of better DFS and OS.

Conclusions: The results suggest role of $h E N T 1$ expression as a (a) predictive biomarker in TNBC and (b) in the aggressive biology of the tumor. Further studies in larger cohort of breast cancer patients are warranted to understand possible role of hENT1 in chemosensitivity of TNBC.

215 Increase in Tumor-Associated T-Lymphocytes Are of Prognostic Importance in Triple Negative Breast Cancer

J Iqbal, H Matsumoto, AA Thike, PH Tan. Singapore General Hospital, Singapore, Singapore.

Background: The majority of tumor infiltrating lymphocytes (TILs) in solid tumors are of the CD3+ T-cell phenotype, which includes CD4+ helper cells and regulatory T-cells as well as CD8+ cytotoxic T-cell. Compared with other subtypes, the clinical outcome of triple negative breast cancer (TNBC) has been shown to be particularly influenced by TILs. The aim of the study was to assess the prognostic role of CD4, CD8 and CD3 expression related to type, density and distribution of TILs in TNBC. Design: Immunohistochemistry was performed on paraffin-embedded tumor tissue of a 
consecutive cohort of 115 female patients diagnosed with TNBC. Immunohistochemical reactions were performed using monoclonal antibodies against $\mathrm{CD} 3, \mathrm{CD} 4$ and $\mathrm{CD} 8$. The intensity of the lymphocytic infiltrate was scored according to the percentage of positive stained cells and density as: 1 , low intensity; 2 , high intensity infiltrate using the median T-cell count as cut-off. The univariate significance of differences in marker expression was appraised by Pearson's chi-square test. The specific OS and DFS were estimated using the Log-rank method. The significance level was set at 0.05 .

Results: No correlations between CD3, CD4, or CD8 expression and tumor clinical and pathological parameters were observed. CD8 (median=30, 0-77), CD4 (median=9, 0-50), $\mathrm{CD} 3$ (median=79, 5-96). Intensity of CD4 cell is correlated with density of lymphocytic infiltration. Higher $\mathrm{CD} 3$ count shows better OS; but $\mathrm{p}$-value is not significant. However higher $\mathrm{CD} 4 / \mathrm{CD} 8$ ratio shows significantly better $\mathrm{DFS}(\mathrm{p}=0.039)$.

Conclusions: (1) Overall, a T-cell infiltrate provides evidence of prognostic importance of T-cell mediated immune response in TNBC. (2) A higher CD4/CD8 ratio independently provides prognostic information in subsets of TNBC patients. Larger studies are needed to confirm the prognostic role of TILs in TNBC.

\section{Is Axillary Lymph Node Dissection Justified Following a Positive U/S-Guided Axillary Fine Needle Aspiration? A Reappraisal Based on the Z0011 Trial Recommendations}

M Jabbour, G Berjawi, K Charafeddine, M Saadeldine, F Boulos. American University of Beirut, Beirut, Lebanon.

Background: A positive axillary FNA is currently used to select patients for axiilary lymph node dissection (ALND). Recently, the Z0011 trial has shown that breast cancer patients with 2 or less involved axillary sentinel lymph nodes (SLN) have no improved survival with completion ALND. The aim of this retrospective study is two-fold: first to evaluate whether positive axillary FNAs are resulting in unnecessary ALND based on Z0011 recommendations, and second to investigate whether certain axillary sonographic features with a positive FNA are helpful in predicting a subgroup of patients eligible for completion ALND without the need for SLN sampling.

Design: 69 positive axillary FNA cases with corresponding ALND were retrieved from the pathology database (1996-2013) at the American University of Beirut Medical Center. Clinico-pathologic features including age, gender, tumor histology, grade, and size, number of lymph nodes (LN) involved, size of $\mathrm{LN}$ metastasis and prior neoadjuvant chemotherapy were recorded. Axillary U/S characteristics including thick cortex, abnormal lobulation, calcifications, doppler signals and adenopathy were documented. Results: $31 / 69$ (44.9\%) of cases with a positive FNA showed 2 or less involved axillary LNs, 9/69 (13.0\%) had a positive FNA and negative axillary LNs; however 8 of the 9 $(88.9 \%)$ received neoadjuvant chemotherapy. $38 / 69(55.1 \%)$ had 3 or more involved axillary LNs, with a median of 7 positive LNs (range 3-36 out of 10-52). U/S analysis revealed $37 / 69(53.6 \%)$ cases with adenopathy, 14/69 (20.3) thick cortices, $7 / 69$ $(10.1 \%)$ adenopathy and thick cortex, $1 / 69(1.5 \%)$ calcifications, $2 / 69(2.9 \%)$ normal $\mathrm{U} / \mathrm{S}$ and in $8 / 69(11.6 \%) \mathrm{U} / \mathrm{S}$ imaging information was not available. A significant correlation between involved LN number $(>2$ or $\leq 2)$ and primary tumor size was identified $(\mathrm{p}=0.001)$. Furthermore, involved $\mathrm{LN}$ number $(>2$ or $\leq 2)$ was associated with metastatic $\mathrm{LN}$ deposit size with a cut-off limit of $0.9 \mathrm{~cm}(\mathrm{p}=0.008)$. Finally, there was no significant predictive value of axillary LN U/S findings and number of LNs involved $(>2$ or $\leq 2) \quad(\mathrm{p}=0.130)$.

Conclusions: The results suggest that axillary LN U/S findings in patients with breast cancer are not predictive of the number of involved LNs and as many as $44 \%$ of patients with a positive axillary FNA will receive an unnecessary ALND based on the Z0011 trial. The sole determinant of completion ALND remains SLN examination with a potential role of tumor size and SLN metastatic size in selecting patients for completion ALND.

217 Phyllodes Tumor (PT) Subsequent to a Diagnosis of Fibroadenoma (FA) on Breast Core Needle Biopsy (CNB): Frequency and Characteristics TW Jacobs, Y-Y Chen, DG Guinee, PR Eby, AA Thike, P Vohra, PH Tan. Virginia Mason Medical Center, Seattle, WA; UCSF, San Francisco, CA; Singapore General Hospital, Singapore, Singapore.

Background: A diagnosis of FA can usually be readily made based on histologic features on CNB, and these patients may be managed by observation alone provided imaging studies are concordant. In contrast, patients with PT require surgical excision. Anecdotal examples exist of PT occurring subsequent to a diagnosis of FA on CNB. However the incidence and features of such cases have not been studied in detail.

Design: To address this, we analyzed the frequency and characteristics of cases where PT occurred subsequent to a diagnosis of FA on CNB over a 16-17 year period amongst 3 large breast pathology services in the USA and Singapore. Pathology databases were searched for all FA on CNB, and PT at surgery, and diagnoses were verified. Pathologic features were evaluated, imaging parameters analyzed, and the results were then correlated.

Results: A total of 4163 consecutive FA were diagnosed on CNB over 16-17 years. 16 patients $(0.38 \%)$ had a PT subsequent to a diagnosis of FA on CNB. In contrast, cellular fibroepithelial lesions on CNB were followed by PT in 41-63\% cases (our published data). PT were benign in 14 and borderline in 2. Median PT size was $30 \mathrm{~mm}$ (range 11-105mm), median age at PT diagnosis was 47 years (range $36-70$ years) and interval between the CNB and PT ranged from 0.3-163.4 months (median 4.0 months). PT stromal cellularity ranged from mild to moderate, 7 had heterogeneous cellularity, and 6 had FA-like foci. Amongst 16 FA on CNB, 10 had fragmented cores, 4 were focally mildly cellular and 2 had mitoses (1-2/10HPF). Of 10 cases with imaging, 7 had suspicious findings (rapid growth, irregular border, lobulation, cystic areas or heterogeneity), 5 at time of CNB and 2 on follow-up. All biopsies were ultrasoundguided: 14 with gun, 2 with vacuum; 15 were $14 \mathrm{~g}$ and 1 was $9 \mathrm{~g} ;<5$ cores were obtained in 7 of 16 cases (range: $2-10$ ).
Conclusions: 1) The incidence of PT subsequent to a diagnosis of FA on CNB is extremely low (0.38\%), with most cases categorized as benign. 2) Although PT heterogeneity likely accounted for several CNB-excision discrepancies, no pathologic features on CNB with FA appeared to be prospectively predictive of PT at excision. 3) Suspicious imaging features at time of CNB or on follow-up should prompt consideration for surgical excision. 4) Diagnosing FA on CNB is reliable and safe, provided there is adequate imaging correlation and follow-up.

218 Targeted Sequencing Analysis of Triple Negative Breast Cancer and Correlation with Clinicopathologic Features

K Jakate, E Brogi, H Won, M Akram, M Berger, YH Wen. Memorial Sloan-Kettering Cancer Center, New York, NY.

Background: Information on genetic alterations of triple negative breast cancer (TNBC) remains limited. We used IMPACT (Integrated Mutation Profiling of Actionable Cancer Targets) assay, a targeted sequencing platform performing exon capture of 300 cancer related genes by hybridization followed by next-generation sequencing to analyze genetic alterations in an annotated cohort of TNBC. We then correlated findings with morphology and outcome.

Design: We extracted DNA from paired frozen tumor ( $>75 \%$ tumor content) and normal tissue of 39 TNBC patients (pts) treated at our center. DNA samples (250-500ng) were subjected to targeted sequencing with IMPACT platform. Tumor/normal pairs were sequenced to average depth of $678 \mathrm{x} / 348 \mathrm{x}$ respectively. We used two-tailed student $\mathrm{t}$-test for continuous variables and Fischer Exact test for categorical variables.

Results: All pts were female, with mean age 47 years (y) (range 28-78); 7 pts had $B R C A 1$ germline mutation. TNBC consisted of $32(82 \%)$ invasive ductal carcinomas NOS (IDC NOS), $5(13 \%)$ apocrine BCs, and $2(5 \%)$ metaplastic BCs. Twenty $(51 \%)$ pts had lymph node (LN) metastases at presentation. Distant metastases (DM) developed in $28 \%$ pts. Median follow-up was 43 months and 11 pts died of disease within 3 y of diagnosis. Average somatic mutations, copy number gains and losses were $3(0-10)$, $17(0-44)$ and $7(0-45)$ respectively. Most frequent alterations are listed in Table 1. Pts with apocrine BC were older than pts with IDC NOS (64 vs $43 \mathrm{y}, \mathrm{p}=<.01$ ). Among 5 apocrine BCs, 3 had PIK3CA mutation, 1 had PIK3RI mutation. Compared with IDC NOS, apocrine BCs had less copy number gains ( 4 vs $19, \mathrm{p}=.006)$ and more PIK3CA pathway mutations $(80 \%$ vs $3 \%, \mathrm{p}<.001)$. Albeit not significant due to small sample size, other trends were: 1) BRCAl pts had more copy number losses (12 vs 6), ARIDIB mutations ( $33 \%$ vs none) and fewer $M Y C$ gains ( $14 \%$ vs $47 \%$ ); 2 ) $M L L 2$ mutations were more frequent in $\mathrm{LN}$-negative pts ( $26 \%$ vs $5 \%$ ) and pts without DM ( $25 \%$ vs none); 3 ) Pts with $<3$ y survival had more frequent copy number gains in PARPI ( $50 \%$ vs $17 \%$ ) and $W N K l(60 \%$ vs $27 \%)$.

\begin{tabular}{|c|c|c|c|c|c|c|}
\hline & & & & & & \\
\hline Rank & Mutation & $\%$ & Gain & $\%$ & Loss & $\%$ \\
\hline$\frac{1}{2}$ & $\sqrt{11553}$ & $\sqrt{74}$ & $\mid$ MYC & $\frac{41}{38}$ & CDKN2A & $\frac{18}{\sqrt{18}}$ \\
\hline 3 & PI3KCA & 10 & \begin{tabular}{|l|} 
NBN \\
N
\end{tabular} & 35 & CDKN2B & 15 \\
\hline 4 & KDM6A & 10 & WNK1 & 34 & PLK2 & 15 \\
\hline 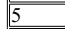 & ARID1B & 10 & DDR2 & 31 & TEK & $\sqrt{15}$ \\
\hline 6 & $\widehat{S Y K}$ & 10 & $\overline{\mathrm{FH}}$ & 31 & FAT1 & 14 \\
\hline$\sqrt{7}$ & JAK1 & 8 & KLF6 & 31 & INPP4B & 10 \\
\hline 8 & MLL3 & 8 & NCOA2 & 28 & BRCA1 & 10 \\
\hline & FAT1 & 7 & PARP1 & 28 & DIS3 & 10 \\
\hline 10 & GRM3 & 7 & PREX2 & 28 & FBXW7 & 10 \\
\hline
\end{tabular}

Conclusions: Frequent mutations detected by IMPACT platform are comparable to TCGA data. Apocrine BC show high frequency of PIK3CA pathway mutations, suggesting PI3K inhibitors may be beneficial in this subgroup of TNBC.

219 Molecular Abnormalities Differentiating DCIS Lesions with and without Associated Stromal Invasion

G Jin, J Groth, J Geradts. Duke University Medical Center, Durham, NC.

Background: We recently described a panel of 8 candidate genes with differential copy number aberrations (CNAs) in microdissected low grade (LG) DCIS cases with and without associated stromal invasion (Liao et al., Genes Chrom. Cancer 51: 1067$78,2012)$. We now report new validation studies on non-microdissected $L G$ and high grade (HG) DCIS lesions.

Design: We selected two cohorts of LG and HG DCIS (50 cases each) that were stratified by the presence of associated invasive carcinoma. DNA was extracted from whole paraffin sections of DCIS and 10 normal control tissues. Quantitative (Q-) PCR analysis was performed as previously described. DNA copy number was additionally assessed by chromogenic in situ hybridization (CISH) of the LG DCIS cohort. Immunohistochemical (IHC) assays were developed for all 8 genes and were applied to the 50 LG DCIS cases. Results: Due to the dilutional effect of DNA from admixed benign cells, many CNAs were not detectable in extracts from whole tissue sections, especially if the tumor cellularity was $<50 \%$. Nevertheless Q-PCR of non-microdissected LG DCIS cases revealed CNA frequencies in SMRT/NCOR2, NR4A1/NUR77 and CELSR1 that were significantly different in lesions with and without invasion. No such differential CNAs were found in HG DCIS. However, HG DCIS had a higher overall frequency of CNAs than LG DCIS in 7 of 8 genes (average 3.4 versus 2.1 per case). Gains of the novel genes GRAP2 and TAF1C and loss of the tumor suppressor gene ST13 were noted in $>40 \%$ of all HG DCIS cases. IHC analysis of the LG DCIS cohort revealed a tendency for more frequent downregulation of DYNLRB2 and overexpression of SMRT/NCOR2 in cases with stromal invasion. There was a high rate of GRAP2 upregulation (65\%) and ST13 downregulation (54\%) across the LG DCIS cohort. 
Conclusions: Contamination of benign DNA affected the detection of CNAs by Q-PCR in whole tissue sections of DCIS. Nevertheless differentially distributed CNAs could be identified in 3 of 8 candidate genes in LG DCIS. Molecular abnormalities in low grade lesions could also be demonstrated by in situ techniques (IHC and CISH). HG DCIS cases had higher CNA frequencies that did not correlate with stromal invasion, however.

220 Tumor MicroEnvironment of Metastasis (TMEM) Is a Novel Approach to the Assessment of Metastatic Risk in Breast Cancer

J Jones, XXue, H-M Lin, T D'Alfonso, P Ginter, M Oktay, B Robinson, F Gertler, A Glass, J Sparano, J Condeelis, TRohan. Einstein, Bronx, NY; Mount Sinai, New York, NY; Weill Cornell, New York, NY; MIT, Boston, MA; Kaiser Permanente, Portland, OR. Background: Although hematogenous metastasis is the major cause of death in breast cancer, current assessments of metastatic risk (OncotypeDx;IHC4) are indirect. TMEM(Tumor MicroEnvironment of Metastasis) is a mechanism-based assay performed on breast cancer samples which quantitates intravasation sites, and has been shown to be significantly associated with the development of systemic metastasis (Robinson et al, 2009). The assay is based on observations from intravital imaging of mouse models. The purpose of this study was to 1) assess TMEM as a marker of metastatic risk in a population cohort of breast cancer patients, 2) compare TMEM with IHC4 -a proxy for OncotypeDx recurrence score (RS), and 3) evaluate the correlation between TMEM score and OncotypeDx RS in a separate cohort.

Design: The population-based cohort included 259 case-control pairs diagnosed between 1980 and 2000. Cases developed distant metastasis; controls did not. Tissue sections of primary breast cancer samples were subjected to a sequential triple immunostain with CD31 (a vascular marker), CD68 (a macrophage marker) and Mena (an invasive tumor cell marker). Five pathologists, blinded to outcome, evaluated TMEM density over 10 HPF (400x) in areas of high tumor cellularity and tumor vascularity. A TMEM is defined as the direct contact of a vascular endotheial cell with a perivascular macrophage and Mena-overexpressing invasive tumor cell. IHC-4 (ER,PR,Her2,Ki67) was assessed using standard staining and interpretation methods. Analysis was performed for 3 subgroups: ER+/Her2-, Her2+, and triple negative.

Results: TMEM was associated with an almost 3 fold increased risk of metastasis in $\mathrm{ER}+/$ Her2- tumors (multivariate $\mathrm{OR}(95 \% \mathrm{CI})$ high vs. low tertile $=2.70(1.39-5.26)$, Ptrend $=0.004)$. IHC4 had only a borderline positive association with risk in this subgroup. The association for TMEM persisted after adjustment for IHC4. TMEM was not associated with lymph node count $(0-4+)$. TMEM score was only weakly correlated with IHC score (Spearman correlation=0.12). In a separate cohort of 58 cases with known OncotypeDx recurrence scores (RS), the Spearman correlation between TMEM and OncotypeDx RS=0.18.

Conclusions: TMEM score is a novel assay that is positively associated with risk of distant metastasis in women with ER+/Her2- breast cancer and offers insight different from IHC4 and OncotypeDx.

221 Is Frozen Section Evaluation of Reexcision Margins of Value in Preventing Reoperation?

JM Jorns, S Daignault, AJ Wu. University of Michigan, Ann Arbor, MI.

Background: Margin reexcision is the most common indication for reoperation in patients undergoing breast conserving therapy (BCT). A prior study at our institution showed marked reduction in reoperation for margin reexcision following the development of an intraoperative frozen section of margins (FSM) practice on lumpectomy specimens. In this study we examined the value of FSM on reexcision specimens.

Design: An electronic database search (8/09-11/12) identified BCT surgeries for margin reexcision only $(\mathrm{N}=177) .27$ were excluded due to surgery at sites where FSM was unavailable. Margins for FSM were defined as follows: true negative (TN) if called negative $(>0.2 \mathrm{~cm})$ at the time of FS and negative on permanent section (PS) control slides; true positive (TP) if called positive (at ink) or close $(<0.2 \mathrm{~cm}$ ) on FS and confirmed by PS; and false negative (FN) if positive or close margins were identified on control or additionally submitted PS slides but not on original FS slides.

Results: Of 150 reexcision cases with FSM capability 46 (30.7\%) underwent FSM. Patients with and without FSM were similar amongst clinicopathologic features, including need for further reexcision.

\begin{tabular}{|c|c|c|}
\hline & FSM (N=46) & No FSM (N=104) \\
\hline Age (y) & $57.4(38.4-84.5)$ & $56.3(29.8-85.3)$ \\
\hline \multicolumn{3}{|l|}{ Diagnosis (\%) } \\
\hline DCIS & 43.5 & 36.5 \\
\hline IDC & 50 & 51 \\
\hline ILC & 4.3 & 7.7 \\
\hline Other & 2.2 & 4.8 \\
\hline \multicolumn{3}{|l|}{ Grade (\%) } \\
\hline 1 & 23.9 & 29.8 \\
\hline 2 & 43.5 & 44.2 \\
\hline 3 & 32.6 & 26 \\
\hline Size (cm) & $1.1(0.1-3.3)$ & $1.4(0.1-6.3)$ \\
\hline \multicolumn{3}{|l|}{ Multiocality (\%) } \\
\hline DCIS & 39.1 & 38.5 \\
\hline Invasive & 10.9 & 16.3 \\
\hline \multicolumn{3}{|l|}{ Previous surgery (\%) } \\
\hline Excisional biopsy & 50 & 43.3 \\
\hline Lumpectomy & 32.6 & 49 \\
\hline Reexcision & 17.4 & 7.7 \\
\hline Previous margin multifocally close/pos (\%) & 56.5 & 51.9 \\
\hline \multicolumn{3}{|l|}{ Rexcision margin status (\%) } \\
\hline No residual & 56.5 & 67.3 \\
\hline Neg & 17.4 & 8.7 \\
\hline Close & 19.6 & 15.4 \\
\hline Pos & 6.5 & 8.7 \\
\hline Rexcision margin multifocally close/pos (\%) & 19.6 & 16.3 \\
\hline Further reexcision needed (\%) & 23.9 & 22.1 \\
\hline \# Surgeries & $1.7(1-4)$ & $1.9(1-4)$ \\
\hline \multicolumn{3}{|l|}{ Final Surgery (\%) } \\
\hline BCT & 82.6 & 84.6 \\
\hline Mastectomy & 17.4 & 15.4 \\
\hline
\end{tabular}

FSM identified $75 \%$ of cases with residual disease (12 TP \& 6 TN margins). FN accounted for the remaining 6 cases, 2 of which did not require reexcision due to extreme focality and limit of anatomic resection. Reexcision in FSM (11/46; 23.9\%) was due to: close/positive margins in specimens sent for PS only(5); pathology sampling error(3); post-op imaging findings(2); and both sampling error \& close/positive margins in specimens sent for PS only(1). FSM was performed most often by 2 of 7 breast surgeons. Conclusions: FSM for BCT reexcision specimens was infrequently performed and highly surgeon-dependent. FSM resulted in no difference in need for subsequent reexcision despite good pathology performance, suggesting FSM of reexcision specimens may not be beneficial.

222 Clinical and Radiologic Follow-Up Study for Biopsy Diagnosis of Radial Scar/Radial Sclerosing Lesion without Other Atypia

E Kalife, A Lourenco, M Resnick, M Mainiero, Y Wang. Alpert Medical School of Brown University, Providence, RI.

Background: Radial scars/radial sclerosing lesions (RS/RSL) are distinct lesions sometimes detected by imaging or microscopically in a needle biopsy (NB) performed for other findings. Advances in imaging techniques and larger NB samples have led to increased diagnosis of RS/RSL; the question of how to manage these lesions remains unanswered. Our objective is to determine the incidence of malignancy at surgery or imaging follow-up when RS/RSL is identified on NB in lesions with otherwise benign histology.

Design: A search of the pathology database from 2005-12 yielded 117 NB with a diagnosis of RS/RSL \& no associated atypia [ductal/lobular atypia (ADH, ALH); flat epithelial atypia (FEA), columnar cell atypia (CCA)] or malignancy. The 68 cases with either surgical excision or documented imaging follow-up [6 mo study after RS/RSL, followed by annual imaging examination if negative (BIRAD 1/2)] were included. The RS/RSL was considered "incidental" if the target was calcifications \& "targeted" if imaging revealed a mass/architectural distortion/MRI enhancement.

Results: Of the 68 NB, 40 were stereotactic for calcifications ("incidental") \& 28 were US/MRI-guided ("targeted") for a mass. In the incidental group, 12 had surgical excision; 28 had close imaging follow-up. In the targeted group, 22 had excision; 6 had close imaging follow-up (see Table 1). Ten surgical excisions showed atypia: 8 had negative follow-up imaging (range 1-7 yrs) and 2 were lost to follow-up. One of the 28 "incidental" patients who had imaging follow-up without surgical excision developed contralateral invasive ductal carcinoma after 7 yrs. There were no ipsilateral malignancies.

\begin{tabular}{|l||l|l|}
\hline NB & Follow-up & Outcome (\%) \\
\hline Incidental 40 & Excision 12 & Benign 8 (66.7) \\
\hline \hline & & ADH 2 (16.7) \\
\hline \hline & & ALH 2 (16.7) \\
\hline \hline & & CCA/FEA 0 $(0)$ \\
\hline \hline & Imaging 28 & DCIS or Invasive Carcinoma 0 (0) \\
\hline \hline Targeted 28 & All negative (1-7 yrs) \\
\hline \hline & & Benign 16 (72.7) \\
\hline \hline & & ADH 2 (9.1) \\
\hline \hline & & ALH 0 $(0)$ \\
\hline \hline & & CCA/FEA 4 (18.2) \\
\hline & Imaging 6 & DCIS or Invasive Carcinoma 0 (0) \\
\hline
\end{tabular}

Conclusions: We found no evidence of associated malignancy at surgical excision after diagnosis of RS/RSL in either study group. Patients with atypia identified on excision had negative imaging follow up for 1-7 yrs without development of ipsilateral malignancy. All cases with close imaging follow-up after RS/RSL diagnosis without surgical excision were negative. Our study suggests that imaging follow-up is adequate for those patients with a RS/RSL without evidence of other atypia on NB. 
Prognostic Significance of RICTOR Overexpression in Invasive Mammary Carcinoma: Cytoplasmic Immunoreactivity Independently Predicts Disease Recurrence

BVS Kallakury, KF Afaneh, OR Elkadi, CB Sheehan, CE Sheehan, AB Boguniewicz, JS Ross. Georgetown University Hospital, Washington, DC; Albany Medical College, Albany, NY.

Background: RICTOR is a core protein of the mTOR (mammalian target of rapamycin) pathway complex which plays a major role in cancer development and progression. Although RICTOR expression has been reported to be associated with increased risk of metastasis in mammary carcinoma, its overall prognostic significance in the disease has not been fully elucidated.

Design: Formalin-fixed, paraffin-embedded tissue sections from 92 cases of invasive mammary carcinoma [74 ductal carcinomas (IDC) and 18 lobular carcinomas (ILC)] were immunostained by a manual method using mouse monoclonal RICTOR (ab56578; Abcam, Cambridge, MA). Cytoplasmic and nuclear immunoreactivity was semiquantitatively scored based on staining intensity and distribution and the results were correlated with morphologic and prognostic variables.

Results: Cytoplasmic RICTOR (cRICTOR) overexpression was observed in 38/92 $(41 \%)$ tumors and correlated overall with high tumor grade $(\mathrm{p}=0.022)$, advanced stage $(\mathrm{p}=0.038)$, ER- status $(\mathrm{p}=0.029), P R-$ status $(\mathrm{p}=0.028)$, disease recurrence $(\mathrm{p}=0.003)$ and with a trend toward HER $2+$ status $(\mathrm{p}=0.10)$. Within the IDC subgroup, $\mathrm{cRICTOR}$ correlated with high tumor grade $(\mathrm{p}=0.04)$, ER- status $(\mathrm{p}=0.022), P R-$ status $(\mathrm{p}=0.031)$, disease recurrence $(\mathrm{p}=0.024)$ and with a trend toward advanced stage $(\mathrm{p}=0.076)$ and HER2+ status ( $\mathrm{p}=0.094)$. Within the ILC subgroup, cRICTOR correlated with disease recurrence $(\mathrm{p}=0.024)$. Within the ER+ subgroup, cRICTOR correlated with high tumor grade $(\mathrm{p}=0.008), P R$ - status $(\mathrm{p}=0.021)$, disease recurrence $(\mathrm{p}<0.0001)$ and with a trend toward advanced tumor stage $(\mathrm{p}=0.053)$. Nuclear RICTOR (nRICTOR) overexpression was observed in 36/92 (39\%) tumors and correlated with low tumor grade within the $\mathrm{ER}+$ subgroup; and with a trend toward correlation with low tumor grade overall $(\mathrm{p}=0.067)$ and within the IDC subgroup $(\mathrm{p}=0.079)$. There was an inverse correlation of cRICTOR and nRICTOR ovexpression $(\mathrm{p}<0.0001)$. On multivariate analysis, early age at diagnosis $(\mathrm{p}=0.002)$, advanced stage $(\mathrm{p}=0.002)$ and cRICTOR $(\mathrm{p}=0.003)$ independently predicted disease recurrence.

Conclusions: Cytoplasmic RICTOR overexpression is associated with adverse prognosis in both IDC and ILC and is an independent predictor of disease recurrence. Further study of RICTOR expression and its potential as a target of therapy for invasive breast cancer appears warranted.

224 Prognostic Significance of Immunohistochemical (IHC) Expression of DC-SCRIPT in Mammary Carcinomas

BVS Kallakury, G Niu, AB Boguniewicz, CB Sheehan, CE Sheehan, JS Ross. Georgetown University Hospital, Washington, DC; Albany Medical College, Albany, NY.

Background: Nuclear receptors such as estrogen, progesterone and androgen receptors have well established roles in progression of breast and prostate cancers. A dendritic cell specific transcript, DC-SCRIPT, is a transcriptional co-regulator of nuclear receptors influencing prognosis in the above malignancies. Although increased DC-SCRIPT mRNA levels have been found to be an independent predictor of favorable outcome in mammary carcinomas, the prognostic significance of immunohistochemical DCSCRIPT protein expression has not been previously reported.

Design: Formalin-fixed, paraffin-embedded tissue sections from 130 cases of invasive mammary carcinoma [86 ductal carcinomas (IDC) and 44 lobular carcinomas (ILC)] were immunostained by automated method (Ventana Medical Systems Inc., Tucson, AZ) using goat anti-human DC-SCRIPT/ZNF366 antibody (R\&D Systems, Minneapolis, $\mathrm{MN}$ ). Cytoplasmic immunoreactivity was scored based on intensity (weak, moderate, intense) and percentage of positive cells (focal $<=10 \%$, regional $11-50 \%$, diffuse $>50 \%$ ) in tumor, in situ disease when present, and adjacent benign epithelium. An additive index was calculated and then cases were assessed as tumor=benign $(T=B)$, tumor $>$ benign $(\mathrm{T}>\mathrm{B})$ and tumor $<$ benign $(\mathrm{T}<\mathrm{B})$; and in situ=benign $(\mathrm{I}=\mathrm{B})$, in situ $>$ benign $(\mathrm{I}>\mathrm{B})$ and in situ $<$ benign $(\mathrm{I}<\mathrm{B})$. Results were correlated with clinicopathologic variables. Results: Cytoplasmic DC-SCRIPT immunoreactivity was noted in tumor, in situ disease, and adjacent benign glands in all cases with $\mathrm{T}=\mathrm{B}[91 / 130(70 \%)], \mathrm{T}>\mathrm{B}[36 / 130(28 \%)]$, $[\mathrm{T}<\mathrm{B} 3 / 130(2 \%)]$ cases; $\mathrm{I}=\mathrm{B}[56 / 68(82 \%)]$ and $\mathrm{I}>\mathrm{B}[12 / 68(18 \%)]$ cases. Overall DC-SCRIPT dysregulation [T $>$ B + T $<$ B, 39/130 (30\%)] correlated with tumor type [ $41 \%$ ILC vs $24 \%$ IDC, $p=0.052$ ]; early tumor stage [ $42 \%$ early vs $17 \%$ advanced, $\mathrm{p}=0.027]$; and within the IDC subgroup correlated with ER positive status [33\% ER+ vs $13 \%$ ER-, $\mathrm{p}=0.045]$. Within the $68 / 130(52 \%)$ cases with in situ disease present, in all I $>$ B $[12 / 68(18 \%)]$ DC-SCRIPT immunoreactivity was also overexpressed in the tumor $(\mathrm{T}>\mathrm{B}), \mathrm{p}<0.0001)$. On multivariate analysis, advanced stage $(\mathrm{p}<0.0001)$ and disease recurrence $(\mathrm{p}=0.008)$ independently predicted overall survival.

Conclusions: DC-SCRIPT protein is expressed in pre-invasive and invasive mammary carcinomas and is more frequently dysregulated in tumors with more favorable prognosis including ILC, ER+ IDC and early stage carcinomas of both types. Further study of DC-SCRIPT expression in mammary carcinomas appears warranted.

225 Comparative Sensitivities and Specificities of Antibodies to Breast Markers GCDFP-15, Mammaglobin A, and Different Clones of GATA-3

PL Kandalaft, AM Gown. PhenoPath Laboratories, PLLC, Seattle, WA.

Background: GATA-3 is a transcription factor that is crucial to differentiation of many tissues including breast, hair follicles, T-lymphocytes, adipose tissue, kidney, and nervous system. Recent studies have shown GATA-3 to be a very sensitive and specific marker for breast and urothelial carcinomas (CAs). Results in the few published studies to date have demonstrated the sensitivity of GATA-3 in breast CA at $91 \%$ and $100 \%$ for ductal and lobular types, respectively. Given this high rate of sensitivity, we wished to compare the specificity and sensitivity of GATA-3 with the breast-restricted markers GCDFP-15 and mammaglobin A, which have been utilized for many years as the standard immunohistochemical markers for identifying metastatic breast CA, with reported sensitivities in the range of $50-80 \%$.

Design: Paraffin-embedded, formalin-fixed tissue sections of various CAs were utilized: 24 infiltrating ductal and lobular breast CAs (IDC and ILC), 22 triple negative breast CAs (TNBCA), 16 transitional cell CAs (TCC), squamous cell CAs (SCC, 16 head and neck, 22 lung), adenocarcinomas (AdCa, 14 gastric, 14 lung, 16 colon, 18 prostatic), and ovarian CAs (OC; 11 papillary serous, 9 endometrioid). All cases were immunostained with monoclonal antibodies $(\mathrm{Ab})$ to mammaglobin A (Mamma31A5), GCDFP-15 (23A3) and GATA-3 (clones L5O-823 and HG3-31). Each slide was scored utilizing the following semiquantitative scale: 0 staining $=$ negative, $<1 \%=$ rare,$+ 1-25 \%=$ focal,$+ 26-75 \%=$ variably + , and $>75 \%=$ uniformly + .

Results:

\begin{tabular}{|l|l|l||l|l||}
\hline CA type & GATA3 (HG3-31) & GATA3 (LSO-823) & GCDFP-15 & Mammaglobin A \\
\hline \hline TNBCA & $59 \%$ & $86 \%$ & $14 \%$ & $23 \%$ \\
\hline IDC and ILC & $91 \% *$ & $96 \% *$ & $74 \%$ & $57 \%$ \\
\hline TCC & $94 \% *$ & $100 \% *$ & $13 \%$ & $0 \%$ \\
\hline SCC (head \& neck) & $0 \%$ & $0 \%$ & $0 \%$ & $0 \%$ \\
\hline SCC (lung) & $5 \%$ & $0 \%$ & $0 \%$ & $0 \%$ \\
\hline Gastric AdCa & $0 \%$ & $0 \%$ & $0 \%$ \\
\hline Lung AdCa & $0 \%$ & $7 \%$ & $0 \%$ & $0 \%$ \\
\hline Colon AdCa & $0 \%$ & $6 \%$ & $0 \%$ & $0 \%$ \\
\hline Prostate AdCa & $0 \%$ & $11 \%$ & $5 \%$ & $44 \%$ \\
\hline OC papillary serous & $0 \%$ & $0 \%$ & $17 \%$ \\
\hline OC endometrioid & $0 \%$ & $0 \%$ & $17 \%$ \\
\hline
\end{tabular}

* majority uniformly +

Conclusions: The newly described transcription factor GATA-3 showed superior sensitivity in identifying breast CAs (96\% clone L50-823, 89\% clone HGC-31) in comparison to GCDFP-15 (74\%) and mammaglobin A (57\%). Both clones of GATA-3 also showed a significantly superior level of sensitivity in detecting TNBCA $(86 \%$ clone L50-823, 59\% clone HGC-31) in comparison with GCDFP-15 (14\%) and mammaglobin A $(29 \%)$. However, the L50-823 clone of GATA-3 showed lower specificity compared with the HG3-31 clone, demonstrating significant positivity in the setting of SCC. An appropriate immunohistochemical panel is critical in determining whether a metastatic CA represents a breast primary.

226 The Rate of Upgrade for Mammary Atypia in MRI-Guided Core Needle Biopsy: A Study of 108 Cases from Three Different Institutions

$R$ Karabakhtsian, S Sanati, $P$ Kumar, B Reig, X Chen, X Huang, D Wang, S Liu, $T$ Khoury. Montefiore Medical Center, New York, NY; Washington University, St. Louis, MI; Roswell Park Cancer Institute, Buffalo, NY.

Background: The rate of upgrade has been extensively studied in mammographically detected lesions. Breast MRI differs from mammography in many fundamental ways. Its use is increasing and concurrently diagnoses of atypia have increased. While the diagnoses of atypia in MRI-guided biopsies have increased, still little is known about the risk of upgrade, mainly due to the relatively low incidence of atypia in MRI guided biopsies.

Design: We reviewed 785 cases to yield $108(13.8 \%)$ cases with atypia over 7-years from three institutions. Mammary atypia included flat epithelial atypia (FEA), atypical ductal hyperplasia (ADH), lobular neoplasia (LN), papilloma, radial scar (RS) or combination of those lesions. The following clinicoradiologic variables were recorded, including age, race, menopausal status, history of breast cancer (concurrent or past, ipsilateral or contralateral), MRI findings [mass-enhancement (ME) or nonmss-enhancement (NME)], and the reason for the MRI (high risk screening, staging, equivocal mammogram, or clinical suspicion). Upgrade was considered when the subsequent surgically excised specimen showed invasive carcinoma (IC) or ductal carcinoma in situ (DCIS). Fisher exact test was used for statistical analysis

Results: Table1 illustrates the risk of upgrade in each category. The rate of upgrade in pure ADH, FEA, LN, papilloma and RS was as follows: 5 of $24(20.83 \%), 0$ of $5(0 \%)$, 5 of $21(23.81 \%), 2$ of $19(10.53 \%)$ and 0 of $10(0 \%)$, respectively, with no significant difference. MRI targeted NME lesions appeared to have higher risk of upgrade compared to ME lesion ( $\mathrm{p}=0.03$ ).

Conclusions: The management of LN diagnosed on mammographically detected lesion with surgical excision is controversial. We found that the risk of upgrade for LN diagnosed on MRI-guided biopsy is comparable to ADH. Therefore, in this setting, LN should be treated with subsequent surgical excision similar to ADH.

Table 1: Correlation of the rate of upgrade and the historadiologic variables

Table 1: Correlation of the rate of upgrade and the historadiologic variables
\begin{tabular}{|l|l|l|}
\hline Variable(total) & Upgrade-Yes & Upgrade-No \\
\hline Pathology & & \\
\hline ADH-Yes(38)* & $9(50)^{\wedge}$ & $29(32)$ \\
\hline ADHH-No(70) & $9(50)$ & $61(68)$ \\
\hline FEA-Yes(14)* & $2(11)$ & $12(13)$ \\
\hline FEA-No(94) & $16(89)$ & $78(87)$ \\
\hline LN-Yes(37)* & $7(39)$ & $30(33)$ \\
\hline LN-No(71) & $11(61)$ & $60(67)$ \\
\hline Papilloma-Yes(29)* & $6(33)$ & $23(26)$ \\
\hline Papilloma-No(79) & $12(67)$ & $67(74)$ \\
\hline RS-Yes(19)* & $1(6)$ & $18(20)$ \\
\hline RS-No(89) & $17(94)$ & $72(80)$ \\
\hline Mass(43)** & $3(17)$ & $40(44)$ \\
\hline Mass like(65) & $15(83)$ & $50(56)$ \\
\hline Hx-Past-ipsilateral-Yes(4)^ & $3(17)$ & $1(1)$ \\
\hline Hx-Past-ipsilateral-No(104) & $15(83)$ & $89(99)$ \\
\hline
\end{tabular}

*includes all cases pure and mixed; ${ }^{\wedge} \mathrm{N}(\%) ; *^{* *} \mathrm{p}=0.03 ; \wedge \wedge \mathrm{p}=0.01$ 


\section{Assessment of Discordances of HER2 Expression and Gene} Amplification: $10 \%$ Versus $30 \%$ Cutoff?

C Karakas, LM McShane, A Sahin, M Yi, S Trivedi, K Hunt, K Keyomarsi. MD Anderson Cancer Center, Houston, TX; National Institutes of Health, Baltimore, MD.

Background: In a rare subgroup of breast cancers defined as HER2+ by immunohistochemistry (IHC) and fluorescence in situ hybridization (FISH) according the $2007 \mathrm{ASCO} / \mathrm{CAP}$ guidelines, HER2 overexpression and gene amplification are restricted to a subset of cancer cells $(>30 \%)$. Some have questioned whether original testing guidelines could be broadened to include more patients to benefit from HER2 targeted agents. The aim of this study was to address two questions: 1) reflex from IHC testing to FISH testing when an initial result of $2+$ has been observed by IHC compared to reflex testing for $2+$ or $1+$ result, 2) use of a threshold of $30 \%$ stained cells to define an IHC score of $3+$ compared to use of a $10 \%$ threshold.

Design: IHC analysis and FISH of HER2 was performed on tissue micro arrays that were collected from US National Cancer Institute's Cooperative Breast Cancer Tissue Resource. Stained TMA cores were assessed for positive cells, and final IHC results on the scale $0,1+, 2+, 3+$ were determined in two different ways using $>30 \%$ and $>10 \%$ thresholds as $3+$ score. All IHC $2+$ cases were reflexed to FISH testing. For purposes of the study, any case that was IHC 1+ was also reflexed to FISH testing. Results were correlated with different clinicopathological variables.

Results: 1000 cases were analyzed by using IHC. FISH were available in 325 cases. Of the 73 cases that were initially $2+$ when using IHC $>30 \%$ cutoff, 24 became $3+$ using the lower IHC $>10 \%$ cutoff. $21 / 24$ ( $87.5 \%)$ of them are amplified with FISH. Of the 49 that stay $2+$ even under the $10 \%$ cutoff, $28 / 49(57.1 \%)$ are FISH amplified. Amplification rate for IHC $1+$ cases were $9 / 252(3.6 \%)(95 \%$ CI $0.02-0.07)$. There was an increased HER2 amplification rate in IHC $1+$ cases especially in tumors with negative PR status and high pathologic grade ( $\mathrm{p}$ value $<0.5$ ). There was no significant change in HER2 positivity with age, nodal status, tumor stage and ER status.

Conclusions: This study indicates that using $>30 \%$ cutoff may cause false negative results of HER 2 status. In addition to current algorithm that recommends FISH to detect IHC $2+$ cases, we suggest considering FISH testing in IHC $1+$ cases especially with adverse prognostic characteristics.

228 Expression of Autophagy-Related Marker Light Chain 3 in Mucinous Carcinomas of the Breast

K Kawachi, S Shimizu, T Yokose. Kanagawa Cancer Center, Yokohama, Japan.

Background: Autophagy is the degradation system of intracellular components via lysosomes and is important for cancer cell survival for satisfy metabolic demand of cancer cells.Although the role of autophagy-related proteins in breast cancer has not been assessed enough and there are few studies that investigated expression of autophagyrelated marker in invasive breast carcinomas of special subtypes. The aim of this study was to assess the expression of autophagy components light chain (LC) 3 in mucinous carcinomas of the breast, major invasive carcinomas of special subtypes, to investigate the relationship between expression of this marker and pathological characters.

Design: Surgically resected mucinous carcinomas of the breast were histologically subclassified into pure (type A or B), or mixed variants. ER, PgR and HER2 immunostaining were evaluated. Immunohistochemical staining for LC3 was performed using formalin-fixed paraffin-embedded tissues of mucinous carcinomas. Biopsied specimens of ER-positive invasive carcinomas of no special subtypes were also immunostained as a reference. The interpretation of staining was evaluated on the basis of the proportion of stained cells and the immunostaining intensity. We use Fisher's exact test for the comparison of the results.

Results: Thirty five cases of mucinous carcinomas and 45 cases of ER-positive invasive carcinomas of no specific subtypes were analyzed. All cases of mucinous carcinomas were ER-positive. The expression levels of LC3 in tumors were higher in mucinous carcinoma than invasive carcinomas of no special subtypes $(p=0.012)$. In mucinous carcinomas, expressions of LC3 tended to be high in mixed variant than pure variant. In pure variant, LC3 expression was higher in type B than type A $(p=0.016)$. LC3 expression of the tumors were significantly associated with high nuclear grade (Grade 1 and 2 vs Grade $3 ; p=0.038$ ).

Conclusions: Autophagy-related marker, LC3, is highly expressed in mucinous carcinomas than non-mucinous ER-positive invasive carcinomas of no special subtypes. This result indicates that autophagic activity may be increased in mucinous carcinomas. In addition, high grade carcinomas or mixed variant mucinous carcinomas revealed higher expression levels of LC3. According to this result, the autophagic activity may be associated with relatively poor-prognosis group of mucinous carcinomas.

229 Nomograms to Predict the Likelihood of Upgrade of Atypical Ductal Hyperplasia Diagnosed on a Core Needle Biopsy in Mammographically Detected Lesions

T Khoury, B Turner, X Chen, D Wang, S Kandel, S Liu. Roswell Park Cancer Institute, Buffalo, NY; Rochester General Hospital, Rochester, NY

Background: The standard of care for atypical ductal hyperplasia (ADH) diagnosed on core needle biopsy (CNB) is excisional biopsy (EB). However, overtreatment has been a concern in mammary premalignant lesions. Accurate estimates of the likelihood of upgrade could assist greatly in decision making for further treatment.

Design: A total of $203 \mathrm{ADH}$ cases diagnosed on CNB in mammographically detected lesions and had subsequent $\mathrm{EB}$ were reviewed. The pathologic features of $\mathrm{ADH}$ were assessed with multivariable logistic regression to predict the likelihood of upgrade for these patients. Nomograms were created using histologic pattern, presence or absence of microcalcifications (calcs) in $\mathrm{ADH}$ and/or in benign ducts, number of foci, size of largest focus with complete involvement, total number of cores, number of involved cores, percentage of concurrent flat epithelial atypia, presence of lobular neoplasia or radial scar, mammographic findings (mass vs. calcs), size of the needle, and BI-RAD. Then, corresponding formulas are created to calculate the risk of upgrade. This risk is then divided into low, intermediate and high.

Results: A total of $57(28.1 \%)$ cases had upgrade. The following parameters were statistically significant in the multivariate analysis and indicative of upgrade, calcs in $\mathrm{ADH}(82.4 \%)$, no calcs in benign ducts $(59.7 \%)$, total number of foci [median and range 3 (1-16)], number of involved cores [median and range 2 (1-10)], and size of largest focus with complete involvement [median and range $0(0-3)$ ]. When the targeted lesion is a mass, $52.4 \%$ of these had upgrade. Two useful nomograms (figure) and two corresponding formulas were created. The first nomogram included calcs in ADH, number of foci, number of involved cores and mass vs. calcs. The second nomogram included number of involved cores, size of largest focus with complete involvement and mass vs. calcs.

Conclusions: We have developed user friendly nomograms that used variables easily recognized to calculate the likelihood of upgrade for $\mathrm{ADH}$. These nomograms could assist the treating surgeon in decision making, particularly when the patient is at risk for surgery.

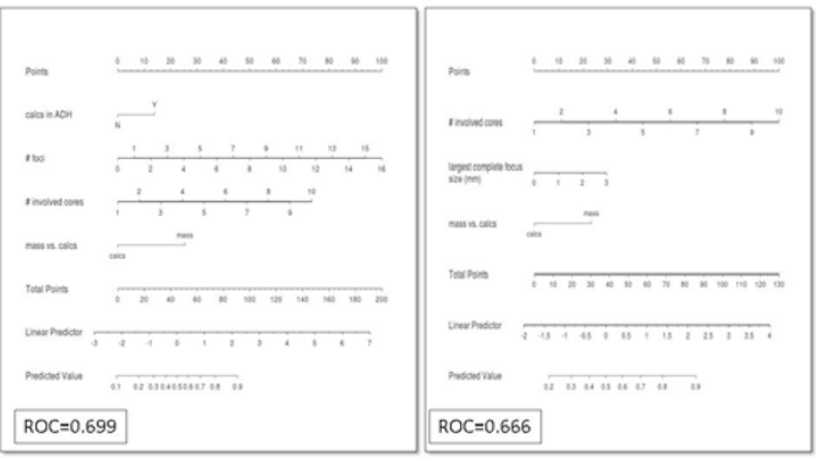

230 Sentinel Lymph Node Frozen Section Utilization Declines in Breast Conserving Therapy Patients after Institutional Policy Change Following the ASCOG Z11 Trial

K Kidwell, JM Jorns. University of Michigan, Ann Arbor, MI

Background: The ASCOG Z11 trial was a prospective randomized trial that found no survival difference between breast conserving therapy (BCT) patients with favorable prognosis breast carcinoma and low volume sentinel lymph node (SLN) metastases that underwent axillary lymph node dissection (ALND) vs. no further surgery. Thus an algorithm was created allowing patients meeting Z11 eligibility criteria to avoid ALND and its comorbidities. This study examined surgeon utilization of SLN frozen section (FS) and outcomes before and after this change.

Design: An electronic database search identified all patients undergoing SLN biopsy 18 months prior to $(\mathrm{N}=116)$ and following $(\mathrm{N}=135)$ institutional policy change. Clinicopathologic features were assessed and data analyzed by a statistician.

Results: Pre- and post-Z11 populations had similar distributions for age, laterality, diagnosis, tumor grade, hormone receptor status, preoperative axillary ultrasound (US) use, SLN procedure and final surgery and FS performance was equivalent. There was a marked decrease in SLN FS use post-Z11 in BCT patients but no difference for those in which SLN was performed alone or with mastectomy.

\begin{tabular}{|c|c|c|c|}
\hline & Pre-Z11 & Post-Z11 & P-value \\
\hline SLN alone & $40 \%(4 / 10)$ & $30.4 \%(7 / 23)$ & 1.59 \\
\hline SLN w/BCT & $73.6 \%(53 / 72)$ & $25 \%(19 / 76)$ & $<.0001$ \\
\hline SLN w/mast & $64.7 \%(22 / 34)$ & $75 \%(27 / 36)$ & .35 \\
\hline
\end{tabular}

Post-Z11 ALND significantly declined for patients with SLN macrometastases ( $\mathrm{p}=.047)$. There was no difference in preoperative axillary US use or need for additional surgery for ALND.

\begin{tabular}{|c|c|c|c|}
\hline & Pre-Z11 & Post-Z11 & P-value \\
\hline Pre-op axillary US: & $\mathrm{N}=116$ & $\mathrm{~N}=135$ & 16 \\
\hline Not done (ND) & $55.2 \%$ & $65.9 \%$ & \\
\hline Negative (neg) & $39.6 \%$ & $26 \%$ & \\
\hline Susp/FNA neg & $5.2 \%$ & $5.9 \%$ & \\
\hline Susp/FNA QNS/ND & $0 \%$ & $2.2 \%$ & \\
\hline ALND performed & $17.2 \%(17 / 116)$ & $11.5 \%(14 / 135)$ & .23 \\
\hline \multicolumn{4}{|l|}{ \#ALND by SLN metastasis size: } \\
\hline Isolated tumor cells & $0 \%(0 / 1)$ & $0 \%(0 / 3)$ & 1 \\
\hline Micrometastasis & $44.4 \%(4 / 9)$ & $23.1 \%(3 / 13)$ & 38 \\
\hline Macrometastasis & $92.9 \%(13 / 14)$ & $57.9 \%(11 / 19)$ & .047 \\
\hline ALND positive & $17.6 \%(3 / 17)$ & $28.6 \%(4 / 14)$ & .67 \\
\hline Separate procedure for ALND & $47.1 \%(8 / 17)$ & $50 \%(7 / 14)$ & .87 \\
\hline
\end{tabular}

SLN positivity correlated with larger tumor size (by imaging $(\mathrm{p}<.0001)$ and pathologic $(\mathrm{p}=.0003)$ staging) and use of preoperative axillary US ( $\mathrm{p}=.003)$.

Conclusions: There was a marked decrease in SLN FS utilization for BCT patients following the post-Z11 policy change with no difference in reoperation for ALND. These findings support the benefit of preoperative axillary staging via US \pm FNA and the reduced need for SLN FS in a post-Z11 era. 
231

Using Immunohistochemical Proliferative Markers to Predict Local Recurrence/Progression of Ductal Carcinoma In-Situ: A Search for a More Cost-Effective Solution

MR Kilgore, OH Chang, S Mehri, PE Swanson, RA Schmidt, ER Parrilla Castellar, RL Garcia, MH Rendi. University of Washington, Seattle, WA.

Background: Ductal Carcinoma In-Situ (DCIS) of the breast is a heterogenous in-situ neoplastic process. Determining which of these lesions will recur locally or progress to invasive carcinoma has proven unreliable. Molecular methods, such as the Oncotype DX ${ }^{\circledR}$ Breast Cancer Assay for DCIS Patients (Genomic Health ${ }^{\mathrm{TM}}$ ), have emerged to predict the likelihood of a "local event" (ipsilateral recurrent DCIS \&/or invasive carcinoma) at 10 years in patients diagnosed with DCIS and treated with local excision. However, these genomic assays are expensive. We examine if more cost effective methods, mainly in-house immunohistochemical assays, can predict the probability of a local event in patients with DCIS

Design: Cases were identified through an initial query to find DCIS patients, without invasive carcinoma, having a follow-up breast case at least 1 year after the first DCIS diagnosis. Reports and slides were then reviewed to arrive at a dataset of 7 patients with local events (positive patients [PP]) at least 1 year out from diagnosis. Negative controls (NC) were defined as patients with at least 10 year follow up in our institution without a local event. PP and NC were matched by DCIS grade and age at diagnosis. Immunohistochemistry ( $\mathrm{IHC}$ ) for Phosphohistone-H3 (pHH3) and Ki67 were performed on all cases. One person, blinded to the cases, counted all cells for consistency. Positive staining was defined as any nuclear stain regardless of intensity. 1000 cells/IHC-stained slide were counted from an area interpreted as the most proliferative.

Results: Across grades, $\mathrm{pHH} 3$ in PP vs NC is statistically significant $(\mathrm{p}<.03)$ and Ki67 approaches significance $(\mathrm{p}<.06)$. For $\mathrm{PP}, \mathrm{pHH} 3$ vs grade is statistically significant $(\mathrm{p}<.02)$ and Ki67 vs grade approaches significance $(\mathrm{P}<.07)$. For $\mathrm{NC}$, there is no relationship between grade and $\mathrm{pHH} 3(\mathrm{p}<.31)$ or Ki67 $(\mathrm{p}<.22)$

Pos Patients (left) : Neg Controls (right)

\begin{tabular}{|c|c|c|c|c|c|c|}
\hline Grade & Age & Ki67+Cells & $\mathrm{pHH} 3+$ Cells & Age & Ki67+Cells & pHH3+ Cells \\
\hline High & 38 & 363 & 22 & 39 & 17 & 0 \\
\hline High & 48 & 239 & 25 & 49 & 178 & 12 \\
\hline Int & 44 & 89 & 5 & 41 & 48 & 0 \\
\hline Int & 49 & 246 & 11 & 35 & 0 & 0 \\
\hline Int & 35 & 108 & 4 & 33 & 83 & 5 \\
\hline Int & 38 & 47 & 3 & 41 & 48 & 0 \\
\hline Low & 30 & 91 & 5 & 37 & 7 & 1 \\
\hline
\end{tabular}

Conclusions: In this small study, DCIS with a follow up local event is associated with increased $\mathrm{pHH} 3$ and Ki67 staining. In positive patients, proliferative rate by $\mathrm{pHH} 3$ staining correlates with grade, which could be part of a multivariate score predictor. Findings suggest IHC may serve as an alternative for more expensive ancillary testing. Additional studies are needed for increased statistical power.

232

Diagnosis of Phyllodes Tumors on Core Needle Biopsy of the Breast: Challenges and Helpful Features

D Kokh, S Kesmodel, T Barry, L Goicochea, KC Tuttle, OB Ioffe. University of Maryland, Baltimore, MD

Background: Accurate preoperative diagnosis is essential for appropriate surgical management of fibroepithelial tumors as fibroadenomas (FA) and phyllodes tumors $(\mathrm{PH})$ require different surgery. We have undertaken a study of these tumors on core biopsy to facilitate surgical triage and avoid additional surgery for positive margins.

Design: Forty patients with $\mathrm{PH}$ were identified in our database. 20 patients with usual FA were used as control group. Core biopsy was primary means of diagnosis in 16 patients with PH; of these, biopsy correctly classified the lesion in 4 cases: 3 as malignant and 1 as borderline, all confirmed on excision. 12 biopsies were diagnosed as cellular FA/ atypical fibroepithelial lesion; excision was recommended. Excision showed benign, borderline and malignant $\mathrm{PH}$ in 5,6 and 1 cases, respectively. Re-excision was necessary for positive margins in 4 patients. Ki-67, CD117, p16 and CD34 were performed; Ki-67 index was determined by digital image analysis (Aperio ScanScope ${ }^{\circledR}$ )

Results: Patients with phyllodes tumors were older than patients with FAs (average age 41.5 vs. 33 ; range $25-59$ vs 20-53), NS. Average tumor size was 3.6 for $\mathrm{PH}$ (0.9$11.2 \mathrm{~cm})$ and $2.5 \mathrm{~cm}$ for FA $(0 .-3.8 \mathrm{~cm}), \mathrm{p}=.02$. Periductal condensation was present in $44 \%$ of PH. More than mild hypercellularity and border infiltration were observed in $38 \%$. Heterogeneity was more reliable; present in all but 1 case of PH. Leaf-like architecture could be appreciated in $62 \%$. Necrosis was seen in 2 malignant PH. None of these features were seen in FA. PASH-like stroma was present in $32 \%$ of FA, in most BN and borderline cases, and in none of malignant PH. Mitotic count was low and therefore not helpful in raising suspicion for BN or borderline tumors, but was elevated in all but one malignant $\mathrm{PH}$ (one case had low mitotic count on core biopsy due to sampling). Ki67 index was $2.9 \%$ in FA, $8.7 \%$ in BN PH, $10.9 \%$ in borderline and $36.6 \%$ in malignant $\mathrm{PH}(\mathrm{p}=.01)$. CD117 was more commonly expressed in borderline and malignant $\mathrm{PH}$, only I one $\mathrm{BN} \mathrm{PH}$, and none FA. p16 and CD34 were not significantly different between any diagnostic entities.

Conclusions: Accurate diagnosis of $\mathrm{PH}$ is difficult on core biopsy material, primarily due to heterogeneity of these lesions and resultant sampling error. Stromal heterogeneity was the most helpful feature on H\&E; periductal condensation, hypercellularity and infiltration were only helpful in some biopsies. CD117 was only helpful in higher grade lesions. Ki-67 index by image analysis most accurately predicted the diagnosis in these limited specimens.
233 Breast Cancer in HIV-Positive Women: Clinicopathologic Features and Comparison with General Population

D Kokh, P Rosenblatt, L Goicochea, S Kesmodel, S Chumsri, D Riedel, OB Ioffe. University of Maryland, Baltimore, MD

Background: In recent decades, women have become the fastest growing group of new HIV cases; since breast cancer is the most common malignancy in women in the United States, the subset of HIV-positive women diagnosed with breast cancer is expected to grow, especially with the use of highly active antiretroviral therapy. Currently, the available data on pathological characteristics and clinical presentation of breast cancer among patients infected with HIV is limited.

Design: 5,000 charts of HIV positive patients diagnosed from 2000-2011 were reviewed, and 20 cases of HIV-positive women with breast cancer were identified. These tumors were then reviewed and compared to 100 consecutive cases from 2011 (historical controls), as well as 20 age- and grade-matched controls, both from our institution.

Results: please see Table 1

\begin{tabular}{|c|c|c|c|c|}
\hline & HIV patients & $\begin{array}{l}\text { Matched } \\
\text { controls }\end{array}$ & $\begin{array}{l}\text { Historical } \\
\text { Controls }\end{array}$ & p value \\
\hline Median age at diagnosis & 48 & 49.3 & 57 & .05 \\
\hline Race & $95 \% \mathrm{AA}$ & $65 \% \mathrm{AA}$ & $57 \% \mathrm{AA}$ & .01 \\
\hline Stage I & $45 \%$ & $35 \%$ & $30 \%$ & .05 \\
\hline Stage II & $30 \%$ & $50 \%$ & $40 \%$ & NS \\
\hline Stage III & $25 \%$ & $15 \%$ & $23 \%$ & NS \\
\hline Stage IV & 0 & 0 & $7 \%$ & NS \\
\hline Family History & $47 \%$ & 0 & $15 \%$ & .01 \\
\hline Grade 1 & $21 \%$ & $20 \%$ & $33 \%$ & NS \\
\hline Grade 2 & $21 \%$ & $25 \%$ & $30 \%$ & NS \\
\hline Grade 3 & $58 \%$ & $55 \%$ & $37 \%$ & NS \\
\hline IDC & $75 \%$ & $85 \%$ & $84 \%$ & NS \\
\hline ILC & $5 \%$ & $10 \%$ & $9 \%$ & NS \\
\hline Medullary & $5 \%$ & 0 & 0 & NS \\
\hline Micropapillary & $10 \%$ & $5 \%$ & $4 \%$ & NS \\
\hline Mucinous & $5 \%$ & 0 & $3 \%$ & NS \\
\hline ER pos & $70 \%$ & $85 \%$ & $81 \%$ & NS \\
\hline PR pos & $50 \%$ & $70 \%$ & $65 \%$ & NS \\
\hline HER2 pos & $20 \%$ & $10 \%$ & $14 \%$ & .07 \\
\hline Luminal A & $20 \%$ & $20 \%$ & $23 \%$ & NS \\
\hline Luminal B & $55 \%$ & $70 \%$ & $59 \%$ & NS \\
\hline HER2 enriched & $5 \%$ & 0 & $4 \%$ & NS \\
\hline Basal-like & $20 \%$ & $10 \%$ & $14 \%$ & .05 \\
\hline LVI & $35 \%$ & $25 \%$ & $19 \%$ & .06 \\
\hline multifocal/multicentric & $35 \%$ & $10 \%$ & $12 \%$ & .05 \\
\hline Lymphocytic peritumoral infiltrate & $35 \%$ & $20 \%$ & $18 \%$ & .07 \\
\hline Lymphocytic mastitis & $35 \%$ & $15 \%$ & $18 \%$ & .07 \\
\hline Hyalinized tumor stroma & $25 \%$ & $5 \%$ & $8 \%$ & .05 \\
\hline LN hyperplasia & $35 \%$ & $10 \%$ & $21 \%$ & .08 \\
\hline Follicle lysis & $25 \%$ & 0 & 0 & 1.02 \\
\hline LN atrophy & $30 \%$ & 0 & $1 \%$ & .01 \\
\hline
\end{tabular}

Conclusions: HIV-positive women in our series have been presenting at a younger age and with family history of breast cancer. These women are more commonly African-American, reflecting the rates of HIV infection in the population. Tumors in these patients tend to possess unfavorable characteristics such as high histologic grade, more common Her2 positivity, multifocal/multicentric distribution, lymphatic vascular invasion, and more prevalent presumed basal-like intrinsic molecular subtype. However, despite these unfavorable predictors, most patients presented with early stage disease; only $25 \%$ had stage III disease and there were no metastatic presentations. Interestingly, both tumors and benign breast tissue in HIV-positive women tend to show more common lymphocytic infiltrate. Both enlarged and atrophic lymph nodes were more common, consistent with HIV lymphadenopathy.

234 Effects of Prolonged Fixation Time on Ki67 in Breast Carcinoma Z Kos, PA Williams, WR Parks, SJ Robertson. University of Ottawa, Ottawa, ON, Canada; Eastern Ontario Regional Laboratory Association, Ottawa, ON, Canada.

Background: Ki67 is emerging as a prognostic and predictive biomarker in breast carcinoma. In routine clinical practice, Ki67 will likely be done on the same tissue as hormone receptors and follow the corresponding fixation guidelines. Long holiday weekends can make it difficult to ensure compliance with the published maximum fixation time of 72 hours. We have previously validated extending fixation time up to 100 hours in our institution for breast prognostic markers. We now investigate the effect of prolonged fixation times on Ki67 counts in breast carcinoma.

Design: Cancer tissue was prospectively collected from 80 surgical resections, with cold ischemic times of less than 60 minutes. Samples from each specimen were fixed in 10\% neutral buffered formalin for $8,12,24,72,100$ and 120 hours; then embedded into the same paraffin block in random order. Ki67 testing was thus done with all variables other than fixation time held constant. Immunostaining was performed on the BOND MAX system using Leica clone MM1. Slide assessment was done blindly; 1000 contiguous tumour cells were manually counted from the visually-identified highest-staining area on each sample using scanned images and tallying annotation software. Results were expressed as the percentage of tumour cells showing any degree of nuclear staining. Results: 50 cases had adequate tumour in the $24 \mathrm{~h}$ fixation sample, considered the gold standard fixation time. Mean Ki67 proliferation indices of samples fixed for 8-120 h showed no statistical difference compared to those fixed for $24 \mathrm{~h}$ (paired t-tests; $\mathrm{p}$-values $0.38-0.67)$. Although there was variation in Ki67 counts amongst the differentially-fixed samples of the same tumour, no trends suggestive of signal degradation emerged. The mean Ki67 scores for specimens fixed for 8-120 h were all within 1.5\% (absolute difference) of those fixed for $24 \mathrm{~h}$, indicating that the variation in Ki67 counts between samples of the same tumour was due to proliferative heterogeneity and not related to fixation time-dependent protein degradation. 
Conclusions: Current recommendations for fixation time for breast hormone receptors are more than adequate for Ki67, the fixation time for which can be safely extended to 120 hours.

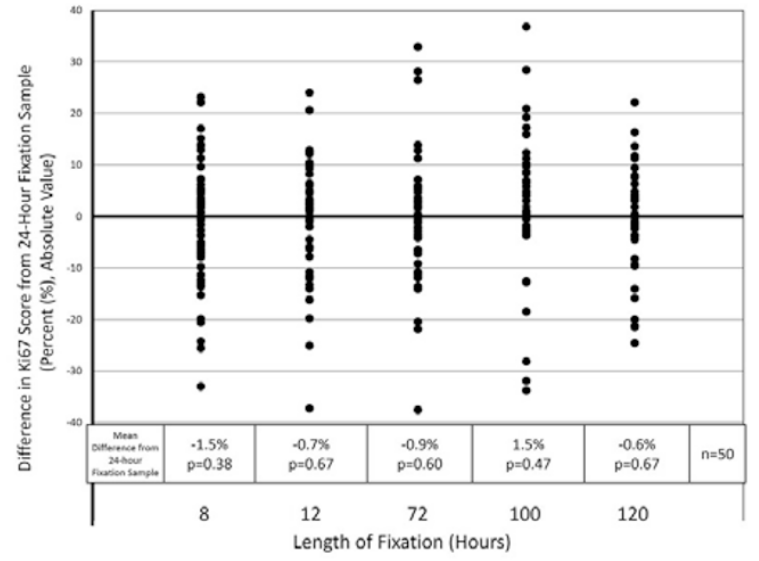

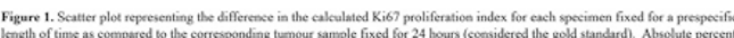

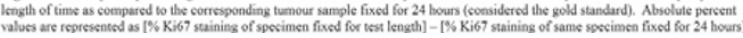

235 Sensitivity and Utility of Commonly Used GATA3 Antibodies in Triple Negative Breast Cancers (TNBC)

$G$ Krings, $M$ Nystrom, $P$ Vohra, $Y$-Y Chen. UCSF, San Francisco, CA.

Background: TNBC are aggressive breast cancers $(\mathrm{BrC})$ which may not express many breast-specific markers. GATA3 expression is increasingly used for diagnosis of metastatic $\mathrm{BrC}$ and may have prognostic value. GATA3 is associated with estrogen receptor (ER) expression, but positivity in TNBC has been reported with widely varying sensitivities. The most commonly used GATA3 antibodies from Biocare Medical (B) and Santa Cruz Biotechnology (S) have not been compared in this setting. The diagnostic utility of GATA3 in conjunction with mammaglobin (MG) and GCDFP15 has not been evaluated in TNBC.

Design: Tissue microarrays of 44 ER+HER2-, 32 HER2+ and 136 TNBC were immunostained with MG, GCDFP15, GATA3B and GATA3S and scored $1+(<1 \%), 2+(1-$ $9 \%), 3+(10-49 \%)$ or $4+(>50 \%)$. Three cores were analyzed per antibody. Whole tissue sections (WTS) of 5 TNBC stained with GATA3B and GATA3S were scored similarly. Results: GATA3B and GATA3S were positive in $48 \%$ and $27 \%$ of all TNBC cores, respectively $(\mathrm{p}=0.000)$, and $49 \%$ positive TNBC cores stained more extensively with GATA3B than GATA3S. GATA3B and GATA3S were positive in $64 \%$ and $44 \%$ TNBC $(\mathrm{p}=0.002)$ and $54 \%$ and $36 \%$ MG-GCDFP15- TNBC, respectively $(\mathrm{p}=0.026)$. Multiple positive TNBC cores per case were consistently more likely with GATA3B $(\mathrm{p}=0.000)$, which also showed more diffuse staining in 5/5 WTS. MG, GCDFP15 or GATA3B were positive in $73 \%$ of TNBC compared to $52 \%$ with GATA3S in the panel (Tables). Comparison of GATA3 Antibodies in BrC Subgroups

\begin{tabular}{|c|c|c|c|c|c|c|}
\hline & TNBC & & ER+HER2- & & HER2+ & \\
\hline & $\begin{array}{l}\text { GATA3B, } \\
\text { GATA3S (n) }\end{array}$ & $\mathrm{p}$ & $\begin{array}{l}\text { GATA3B, } \\
\text { GATA3S (n) }\end{array}$ & $\mathrm{p}$ & $\begin{array}{l}\text { GATA3B, } \\
\text { GATA3S (n) }\end{array}$ & $\mathrm{p}$ \\
\hline$\%$ total cores ${ }^{+}$ & $48(318), 27(325)$ & 0.000 & $98(121), 90(121)$ & 0.030 & $94(89), 82(89)$ & 0.018 \\
\hline $\begin{array}{l}\%+\text { cores upscored with } \\
\text { GATA3B }\end{array}$ & $49(86)$ & & $21(109)$ & & $27(73)$ & \\
\hline$\%$ cases $\geq 1$ core + & $64(126), 44(126)$ & 0.002 & $93(44), 96(44)$ & 1.00 & $97(31), 90(31)$ & 1.00 \\
\hline$\%$ cases $\geq 2$ cores + & $45(110), 19(110)$ & 0.000 & $93(41), 90(41)$ & 1.00 & $90(31), 84(31)$ & 1.00 \\
\hline MG-GCDFP15-, $\% \geq 1$ core + & $54(78), 36(77)$ & 0.026 & $88(8), 88(8)$ & 1.00 & $100(4), 100(4)$ & 1.00 \\
\hline MG-GCDFP15-, $\% \geq 2$ cores + & $28(78), 10(77)$ & 0.008 & $88(8), 88(8)$ & 1.00 & $100(4), 100(4)$ & 1.00 \\
\hline $\begin{array}{l}\text { \% GATA3+, MG }+ \text {, or } \\
\text { GCDFP15+ }\end{array}$ & $73(131), 52(131)$ & 0.001 & $98(44), 98(44)$ & 1.00 & $100(32), 100(32)$ & 1.00 \\
\hline
\end{tabular}

GATA3, MG and GCDFP15 in TNBC

\begin{tabular}{|c|c|c|c|c|c|c|c|}
\hline & GA & $3 \mathrm{~S}$ & $p(B \vee S)$ & MG & $\begin{array}{l}\text { p (MG v } \\
\text { GATA3B, } \\
\text { GATA3S) }\end{array}$ & P15 & $\begin{array}{l}\text { p (GCDFP15 } \\
\text { GATA3B, } \\
\text { GATA3S) }\end{array}$ \\
\hline-1 & $64(126)$ & $44(126)$ & .002 & (135) & $0.000,0.007$ & 35) & $0.000,0.000$ \\
\hline $\begin{array}{l}\text { \% TNBC } \geq 2 \\
\text { cores }+(\mathrm{n})\end{array}$ & $45(110)$ & 19(110) & 0.000 & $18(125)$ & $0.000,1.000$ & $13(127)$ & $0.000,0.287$ \\
\hline
\end{tabular}

Conclusions: GATA3B is more technically and clinically sensitive than GATA3S in TNBC, including MG-GCDFP15- cases, and is more technically sensitive in other BrC groups. An immunopanel of MG, GCDFP15 and GATA3B shows optimal sensitivity for TNBC

236 The Rate of Upgrade for Mammary Atypia in MRI-Guided Core Needle Biopsy: A Single Institution Experience

P Kumar, X Chen, X Huang, D Wang, S Liu, T Khoury. Roswell Park Cancer Institute, Buffalo, NY.

Background: Atypia detected after mammary core needle biopsy (CNB) requires surgical excision. The purpose of this study is to evaluate the risk of upgrade in MRI guided biopsy and to define the risk factors that can predict an upgrade.

Design: A total of 358 MRI-guided mammary CNB were reviewed from 2005 to 2013 in a single institution. Mammary atypia included flat epithelial atypia (FEA), atypical ductal hyperplasia (ADH), lobular neoplasia (LN), papilloma, radial scar (RS) or mixed of the abovementioned lesions. The following clinicopathologic variables were recorded, age, race, menopausal status, history of breast cancer (concurrent or past, ipsilateral or contralateral), MRI findings [mass-enhancement (ME) or non-mass-enhancement
(NME)], and reason for MRI (high risk screening, staging, equivocal mammogram). Upgrade was considered when the subsequent surgically excised specimen showed invasive carcinoma (IC) or ductal carcinoma in situ (DCIS). Fisher exact test was used for statistical analysis.

Results: A total of 68 of $358(19 \%)$ had atypia, FEA ( $n=4)$, ADH ( $n=15)$, LN ( $n=16)$, papilloma $(\mathrm{n}=7), \mathrm{RS}(\mathrm{n}=7)$ and mixed $(\mathrm{n}=19)$. Eleven $(16.2 \%)$ cases had upgrade, divided among $\operatorname{ADH}(n=3), L N(n=4)$ and mixed $(n=4)$. There was no statistically significant difference between any of the histologic types in terms of risk for upgrade. Table 1 illustrates the correlation between the variables and the upgrade status.

Conclusions: MRI targeted NME lesions appeared to have higher risk of upgrade compared to ME lesion. While the requirement for surgical excision for mammographically-detected LN is controversial, MRI-detected LN has similar rate of upgrade to $\mathrm{ADH}$. Therefore, we recommend these lesions to be surgically excised. Table 1: Correlation of the rate of upgrade and the historadiologic variables

\begin{tabular}{|c|c|c|}
\hline Variable (total) & Upgrade-Yes & Upgrade-No \\
\hline \multicolumn{3}{|l|}{ Histology } \\
\hline ADH-Yes (25) & $21(37)^{*}$ & $4(36)$ \\
\hline ADH-No (43) & $36(63)$ & $7(64)$ \\
\hline LN-Yes (29) & $22(39)$ & $7(64)$ \\
\hline LN-No (39) & $35(61)$ & $4(36)$ \\
\hline Papilloma-Yes (13) & $11(19)$ & $2(18)$ \\
\hline Papilloma-No (55) & $46(81)$ & $9(82)$ \\
\hline RS-Yes (16) & $15(26)$ & $1(09)$ \\
\hline RS-No (43) & $42(74)$ & $10(91)$ \\
\hline \multicolumn{3}{|l|}{ MRI findings^ ${ }^{\wedge}$} \\
\hline ME (26) & $26(46)$ & $0(0)$ \\
\hline NME (44) & $31(54)$ & $11(100)$ \\
\hline \multicolumn{3}{|l|}{ Reason for MRI } \\
\hline Equivocal mammogram (4) & $4(7)$ & $0(0)$ \\
\hline High risk (41) & $35(61)$ & $6(55)$ \\
\hline Staging (23) & $18(32)$ & $5(45)$ \\
\hline
\end{tabular}

$* \mathrm{~N}(\%) ; \wedge \mathrm{p}<0.01$

237 Unnecessary Subsequent Surgery Might Be Avoided for a Diagnosis of Atypical Ductal Hyperplasia on a Core Needle Biopsy by Evaluating Historadiologic Features: A Study of 67 Cases

P Kumar, X Chen, D Wang, S Liu, T Khoury. Roswell Park Cancer Institute, Buffalo, NY. Background: The standard of care for atypical ductal hyperplasia (ADH) diagnosed on core needle biopsy (CNB) is excisional biopsy (EB). However, overtreatment has been a concern in mammary premalignant lesions. Accurate estimates of the likelihood of upgrade could assist greatly in decision making for further treatment.

Design: A total of 67 consecutive ADH cases diagnosed on CNB in mammographically detected microcalcifications (calcs) and had subsequent EB were reviewed. The pathologic features of ADH were assessed including histologic pattern, presence or absence of calcs in $\mathrm{ADH}$ and/or in benign ducts, number of foci, size of largest focus with complete involvement, total number of cores, number of involved cores, and size of the needle. Pre-excision radiologic type of calcs (round/punctate, amorphous coarse or heterogeneous, and fine pleomorphic or linear branching), distribution (clustered, linear, or regional/segmental/diffuse) and size of the calcs were recorded. Presence or absence of post-excision calcs was also recorded. Upgrade was considered when the subsequent surgically excised specimen showed invasive carcinoma or ductal carcinoma in situ. Fisher exact test was used for statistical analysis.

Results: A total of $18(26.9 \%)$ cases had upgrade. The significant variables are listed in table 1 . There was no statistically significant difference among the various radiologic types of calcs.

Conclusions: Our results indicate that there are historadiologic features that might help in predicting malignant findings on a subsequent EB. These histologic features, along with imaging studies can help clinicians to subcategorize patients into high and low risk groups for malignancy, thus possibly avoiding unnecessary subsequent surgery.

Table1: Statistically significant historadiologic variable predicting upgrade

\begin{tabular}{|c|c|c|c|}
\hline Variable & Upgrade-No & Upgrade-Y & P value \\
\hline \multicolumn{4}{|l|}{ Histology } \\
\hline \#Foci * & $0(0-4)$ & $0(0-16)$ & 0.01 \\
\hline$\#$ \#nvolved cores* & $1(1-5)$ & $2(1-10)$ & 0.05 \\
\hline Size of largest complete focus-mm* & $1.5(0.2-3)$ & $1.9(0.3-3)$ & 0.01 \\
\hline \multicolumn{4}{|l|}{ Radiology } \\
\hline \multicolumn{4}{|l|}{ Distribution of calcs } \\
\hline Clustered ${ }^{\wedge}$ & $36(77)$ & $9(47)$ & 0.01 \\
\hline Regional, segmental or diffuse ${ }^{\wedge}$ & $8(17)$ & $3(16)$ & \\
\hline Linear ${ }^{\wedge}$ & $3(6)$ & $7(37)$ & \\
\hline \multicolumn{4}{|l|}{ Residual calcs } \\
\hline $\mathrm{No}^{\wedge}$ & $16(39)$ & $2(12)$ & 0.06 \\
\hline Yes^ & $25(61)$ & $14(88)$ & \\
\hline
\end{tabular}

*median and range; ${ }^{\wedge} \mathrm{N}(\%)$

$238 \mathrm{C} / \mathrm{EBPb}$ Expression Is an Independent Predictor of Overall Survival in Breast Cancer Patients by CD4-Dependent Mechanism of Metastasis Formation

E Kurzejamska, V Prakash, P Religa. Karolinska Institutet, Stockholm, Sweden; Warsaw Medical University, Warsaw, Poland.

Background: $\mathrm{C} / \mathrm{EBPb}$ is a transcription factor playing a critical role in mammary gland development and breast cancer progression. In this study, we analyzed the prognostic significance of $\mathrm{C} / \mathrm{EBPb}$ expression in invasive breast cancer and determined the effect of $\mathrm{C} / \mathrm{EBPand}$ metastasis formation in murine $4 \mathrm{~T} 1$ tumor model.

Design: Tissue microarray (TMA) study was performed on 173 cases of invasive breast cancer. Using immunohistochemistry, the intensity of $\mathrm{C} / \mathrm{EBPb}$ staining was evaluated and compared with relevant clinicopathological characteristics. Cox proportional 
hazards models and Kaplan-Meier analysis were used to assess the effect of $\mathrm{C} / \mathrm{EBPb}$ expression on overall survival (OS). Additionally, mouse 4T1 tumor model was used in order to determine the effect of C/EBPand metastasis formation.

Results: C/EBP $\beta$ expression analysis of human tissue microarray (TMA) revealed that decreased C/EBP $\beta$ expression is associated with shorter overall survival of breast cancer patients. Studies performed on murine 4T1 breast cancer model showed that $\mathrm{C} / \mathrm{EBP} \beta$ affects tumor growth, its morphology and metastasis formation in the lungs with strong inflammation. This hypothesis was confirmed by means of microarray and immunohistochemical analysis, which showed that C/EBP $\beta$ controls immunological response in murine $4 \mathrm{~T} 1$ tumors via activation of $\mathrm{MHCII}$ and $\mathrm{CD} 4+$ lymphocytes. Furthermore, anti-CD3 and anti-CD4 treatment of $\mathrm{C} / \mathrm{EBP} \beta$-silenced tumor-bearing mice resulted in attenuation of $\mathrm{C} / \mathrm{EBP} \beta$ effect on tumor morphology and its effect on tumor growth. Additionally, experiment on CD4+ cell-deprived nude mice confirmed CD4-dependent mechanism of metastasis formation.

Conclusions: Presented results indicate that $\mathrm{C} / \mathrm{EBPb}$ expression in breast cancer is an independent predictor of $\mathrm{OS}$ in breast cancer patients. The effect of $\mathrm{C} / \mathrm{EBPb}$ on lung metastasis formation is mediated by MHCII expression and accumulation of CD4+ lymphocytes in the tumor

\section{Androgen Receptor Expression with Different Significance in ER} Positive and Negative Breast Cancers

MD Lacambra, JYS Tsang, Y-B Ni, S-K Chan, M-M Shao, BKB Law, PH Tan, GMK Tse. Prince of Wales Hospital, The Chinese University of Hong Kong, Shatin, Hong Kong; Kwong Wah Hospital, Yau Ma Tei, Hong Kong; Shenzhen Affiliated Hospital, Guangzhou University of Traditional Chinese Medicine, Shenzhen, China; Union Hospital, Shatin, Hong Kong; Singapore General Hospital, Singapore, Singapore. Background: Androgen receptor (AR) is a nuclear steroid hormone receptor, similar to estrogen and progesterone that is highly expressed in breast cancers regardless of estrogen receptor status. To date, most studies focus mainly on ER and PR, and thus the role of AR in breast carcinogenesis is less clearly defined.

Design: A total of 1144 primary invasive breast carcinoma were included in this cohort. AR was correlated with different immunohistochemistry (IHC) stains (ER, PR, AR, EGFR, Her2, Ki67, p63, CK5/6, CK14, c-kit, GCDFP-15, and aßcrystallin), and its association with the different molecular subtypes using IHC as surrogate (luminal A, luminal B, Her2 group, basal-like breast cancers, and unclassified) was analyzed. Results: AR expression was associated with lower tumor grade $(\mathrm{p}<0.001)$, presence of extensive in-situ component ( $\mathrm{p}=0.003)$, absence of necrosis $(\mathrm{p}<0.001)$, lower degree of lymphocytic infiltration $(\mathrm{p}<0.001)$, and lower $\mathrm{pT}$ stage $(\mathrm{p}<0.001)$; and not related with lymphovascular invasion, nodal status, and apocrine features. AR expression was seen in $57.8 \%$ ( 461 of 798) of ER positive and 24.7\% ( 84 of 340) of ER negative cancers. The expression rate of AR was $54.7 \%$ in luminal A, 57.6 in luminal B, 28.0\% in Her2+, and $21.6 \%$ in TNBC, $15.3 \%$ in BLBC, and $26.7 \%$ in unclassified. AR is correlated positively with ER ( $p<0.001)$, PR $(p<0.001)$, and GCDFP-15 $(p<0.001)$; but negatively correlated with c-kit $(\mathrm{p}=0.035)$, CK $5 / 6(\mathrm{p}<0.001)$, CK14 $(\mathrm{p}<0.001)$, and $\mathrm{a} \beta$ crystallin $(\mathrm{p}=0.002)$. No significant correlation was found with Her2, EGFR, and p63. A significant association of AR expression with molecular apocrine cancers was also observed $(\mathrm{p}<0.001)$. As to patients outcome, AR expression was associated with better overall survival and disease free survival ( $p<0.001$ respectively).

Conclusions: AR expression with various clinico-pathologic features, biomarker expression, and patient's outcome differed in ER+ and ER- cancers, suggesting a potentially different roles of AR pathway and that AR expression generally bears a more favorable prognostic role. Furthermore, AR in molecular apocrine cancers may have substantial prognostic implication and as such warrants further validation.

240 Clinicopathological Characterization of pT1a,b pNO M0 Breas Cancer - Impact on Treatment Decision in the ODISSEE Cohort

MLacroix-Triki, NRadosevic-Robin, B Louis, I Roche-Comet, M-S Soubeyrand, E Mery, F Dalenc, Y Belkacemi, F Penault-Llorca. Institut Claudius Regaud, Toulouse, France; Centre Jean Perrin and ERTICa EA4677, Clermont Ferrand, France; Cabinet d'Anatomie et Cytologie Pathologiques, Schiltigheim, France; Institut HistoCytoPathologie, Le Bouscat, France; Cabinet Cypath, Villeurbanne, France; AP-HP Centre Sein Henri Mondor, Créteil, France.

Background: Incidence of infracentimeter breast cancer (icBC) is increasing but its management remains controversial. Namely, the role of biomarkers in the decision for systemic adjuvant therapy is not fully explored. The aims of this study were to (i) detail the clinicopathological and molecular characteristics of icBC in a large multicenter cohort and (ii) assess the influence of icBC biopathology on treatment decision.

Design: From 2009 to 2010, 616 patients with unifocal, pT1a,b pN0 M0 BC were included in the ODISSEE cohort. Central review ( $\mathrm{n}=375$, reported herein) was performed independently by two pathologists and discordant cases resolved under a multihead microscope. Immunohistochemical analysis was performed on tissue microarrays for markers of molecular classification (ER, PR, HER2, Ki67, EGFR, cytokeratin 5/6), ER-pathway (Bcl-2, FOXA1, GATA3, COX2, AR) and PI3K/AKT/mTOR pathway (eIF4E, 4EBP1-p, PS6)

Results: Median age was $60 \mathrm{yr}$. Median tumor size was $8 \mathrm{~mm}$ (14\% pT1a, $86 \%$ pT1b). Histological types were: $72 \%$ invasive ductal carcinoma of no special type, $11 \%$ invasive lobular carcinoma and $17 \%$ others (among which 53\% of tubular). Tumors were mainly grade I or II ( $50 \%$ and $40 \%$ respectively), with low mitotic count in $82.5 \%$ and Ki67 index $<14 \%$ in $87.3 \%$. Most tumors were hormone receptor-positive (93\%) and of luminal A subtype (84.6\%); only few were HER2-positive (4.3\%) or triplenegative $(1.5 \%)$. We observed a distinct, molecular subtype-related pattern of signaling pathway marker expression. ER-pathway markers were significantly associated with luminal tumors. On the contrary, higher Ki67 index and increased 4EBP1-p and PS6 expression were statistically associated with non-luminal HER2+ and triple-negative phenotypes and more frequently found in luminal B as compared to luminal A tumors. Decision for systemic adjuvant treatment was based on the presence of an aggressive phenotype such as HER $2+(72.7 \%$ of whom received chemotherapy + trastuzumab $)$ or triple-negative (chemotherapy in $36.4 \%$ ).

Conclusions: The pT1a,b pN0 BC ODISSEE cohort mainly corresponded to lowgrade, ER+, HER2-, luminal A tumors. However, activation of the eIF4E/4EBP1 axis in the PI3K/Akt/mTOR-pathway warrants further investigation to establish its role in the non-luminal subgroup of icBC, whose management still remains controversial.

241 Do Male and Female Breast Cancer Share mRNA Expression Patterns? Similarities and Differences

KLara Otero, C Garcia Maciass, J Sanz, MJ Merino. NCI, Bethesda, MD; University of Salamanca, Salamanca, Spain; Hospital Clinico Universitario, Madrid, Spain.

Background: Mature microRNAs (miRNAs) are small (18-25 nucleotides) non-protein coding RNAs which modulate gene expression by acting post-transcriptionally. There are only few studies suggesting that male breast cancer (MBC) displays distinctive miRNAs patterns with relevance in the development of MBC but whether these MBC have similar expression patterns than female breast cancer (FBC) is not known. We performed a comprehensive profiling of 800 miRNAs in MBC samples to further elucidate their role in the development and treatment of these tumors, and compared them with known profiles in FBC.

Design: Forty-two cases of MBC were studied and their clinicopathological features were reviewed, including tumor size, lymph node metastasis and status of ER, PR, AR, Her-2 and Ki-67. After manual microdissection, miRNA was extracted from tumor and normal epithelium for profiling using NanoString approach. Differentially expressed miRNAs for each case were obtained comparing tumor vs. normal epithelium.

Results: The comprehensive profiling of 800 miRNAs showed different microRNA expression between tumor and normal tissues, suggesting a cancer-specific microRNA expression profile. Forty-nine miRNAs showed increased expressions while 26 were down-regulated in the cancers. The miRNA profile found in our study was different from known miRNA profiles in FBC. For instance, expression of miR-222 and miR296-5p have shown no significant difference between breast cancer and normal tissue in females, while in the present study, both showed to be up-regulated more than two fold in male tumors. miR-135b-5p, miR-1180, miR-503, miR-34a-5p, miR-362-5p, miR424-5p and miR-194-5p showed to be significantly up-regulated in our cohort of male tumors while in female breast cancer these miRNAs have shown to be down-regulated. Among the most down-regulated miRNAs found in the present study, miR-155-5p and miR-203 have shown to be up-regulated in female breast tumors. Relationships between the expression of the most deregulated miRNAs and the pathologic features showed a statistically significant association between tumor size and miR-125b $(P<0.05)$ Conclusions: The results presented in this study support the fact that $\mathrm{MBC}$ has a different miRNA expression profile when compared with female breast cancer, that specific miRNA's may play an important role in the development and progression of $\mathrm{MBC}$, and that they provide new tools for the development of new molecular therapeutic targets.

242 Male Breast Cancer, Is There a Correlation between Prognostic Factors and Molecular Analysis?

K Lara-Otero, C Garcia Macias, J Sanz, MJ Merino. National Cancer Institute, Bethesda, MD; Universidad de Salamanca, Salamanca, Spain; Hospital Clinico Universitario, Madrid, Spain.

Background: Male breast cancer (MBC) is rare with about 2,140 new cases and 500 deaths/year. These cancers share morphological characteristics with its female counterpart, but, male tumors are less common, present at later onset, and have overall poor prognosis. $\mathrm{MBC}$ has a high rate of hormone receptor expression $(\mathrm{ER}+\mathrm{PR}+)$, whereas most tumors do not show Her2 amplification. We investigated the risk factor profile, histologic features and mRNA molecular profile in MBC and investigated whether they have correlation with clinico-pathologic parameters and a possible role in therapy.

Design: Forty-two cases of MBC were studied and their clinico-pathological features reviewed (tumor size, tumor type and the presence of nodal metastasis). Status of ER, PR, Her-2, AR, Ki-67 and p53 was determine by IHC. In 12 cases, normal and tumor tissues were microdissected for RNA extraction and microRNA's quantitative assay for miR10, miR106a, miR-181c, miR-183, miR-17 and miR-21 was done to evaluate mature miRNA expression using the TaqMan ${ }^{\circledR}$ approach with RNU6B as endogenous control. Results: Age range was $36-85$ (m 61 years). Tumor size varied from 0.5 to $5 \mathrm{~cm}$. Histologically, most tumors were invasive ductal cancers, 4 were papillary, 1 micropapillary and 1 mucinous. Fifty-five percent were grade II and $45 \%$ Grade III; six cases were DCIS. LN status was known in $68 \%$ of the cases, of these, $74 \%$ had lymph node metastasis. Prognostic factors were known in 28 cases, 25 were ER+ and $22 \mathrm{PR}+, 2$ were ER+/PR- and 3 had Her2 amplification, 6 were 2+ and the rest were negative. P53 was positive in 8 cases. AR was heterogenous and was strongly + in DCIS. There was down regulation of miR-10a, miR-181c, miR-106a and miR-17. miR-183 was up-regulated. All cases that showed miR-183 up-regulation were ER+/ $\mathrm{PR}+$ and were either moderate or high grade tumors. Although correlation between down-regulation of miR-10a, miR-181c, miR-106a and miR-17 and prognostic factors presented more variability, the tendency was towards a majority of ER and PR positivity and moderate grade tumors.

Conclusions: Our study suggests that male breast cancer has specific miRNAs implicated in the development and progression of male breast cancer. The presence of these miRNAs and their correlation with prognostic factors may identify tumors with aggressive behavior and makes them good candidates as possible molecular targets. 
243 Comparing Definitions of HER2 Gene Amplification and "Heterogeneity" in Breast Carcinoma: A Retrospective Cohort Study $B$ Lategan, $M$ Wood, MC Chang. Mount Sinai Hospital, Toronto, ON, Canada; University of Toronto, Toronto, ON, Canada.

Background: Amplification of human epidermal growth factor receptor 2 (ERBB2/ HER2) is of prognostic and predictive significance in breast carcinoma. HER2 gene amplification is determined by in situ hybridization (ISH). To decide on anti-HER2 treatment, the FDA defines HER2-amplified by HER2/CEP17 ratio $>2.0$ or HER2 copy number $>6$. Because of intratumoral heterogeneity, the percentage of individual HER2+ (\%-cells HER2+) cells sometimes drives treatment decisions. Our aim was to compare different definitions of HER2+.

Design: We identified 222 consecutive cases of primary breast carcinoma tested by ISH for HER2 with follow-up data available (median time $=33 \mathrm{mo}$ ). This included cases tested by ISH only and those reflexed for equivocal IHC. For each case, HER2 and CEP17 counts were recorded, and also \%-cells HER2+. Descriptive statistics and Kaplan-Meier survival analysis were applied.

Results: Based on the FDA (ratio >2.0) definition, 37/222 (17\%) were HER2+. Using HER2 copy number $>6,33 / 222(15 \%)$ were HER2+. Using \%-cells HER2+, thresholds of $10 \%$ and $50 \%$ resulted in HER 2 rates of 56/222 (25\%) and 32/222 (14\%) respectively. On Kaplan-Meier survival analysis, there was a (non-significant) trend for HER2+ groups towards poorer prognosis when using ratio $>2.0$ (and HER2 $>6$, not shown), but not $10 \%$-cells HER2+.

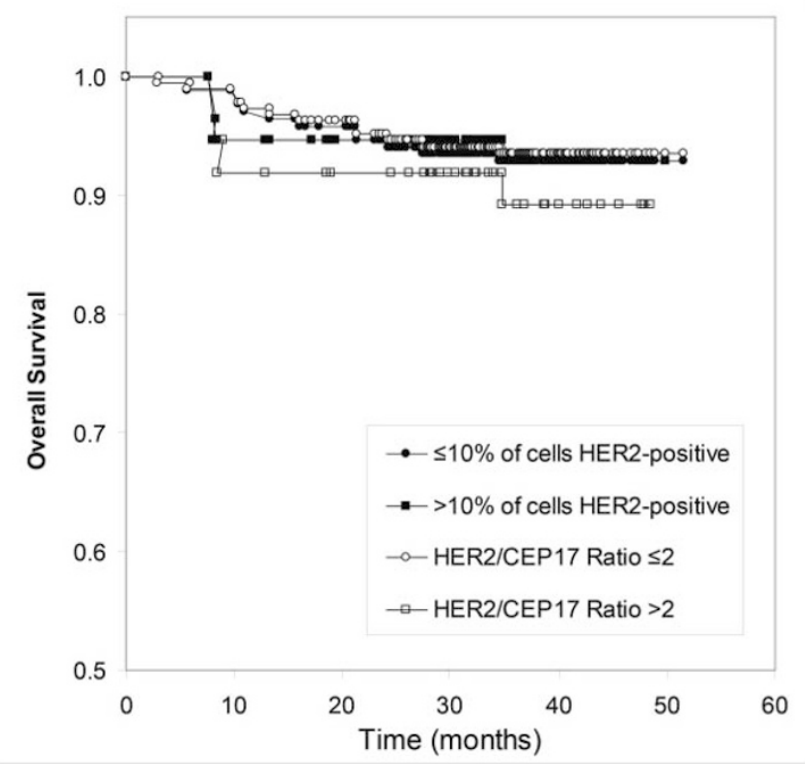

Figure 1: Kaplan-Meier survival analysis shows a (non-significant) trend for the HER2+ group defined by HER2/CEP17 Ratio $>2.0$ towards poorer prognosis, but not the HER2+ group defined by $>10 \%$-cells HER2-positive.

The relation between \%-cells HER2+ and HER2/CEP17 ratio was a standard logistic curve. Using $10 \%$-cells HER2+ as a threshold is equivalent to shifting the HER2/ CEP17 ratio to 1.6, compared to 2.2 at 50\%-cells HER2+ and 2.0 at 30\%-cells HER2+.

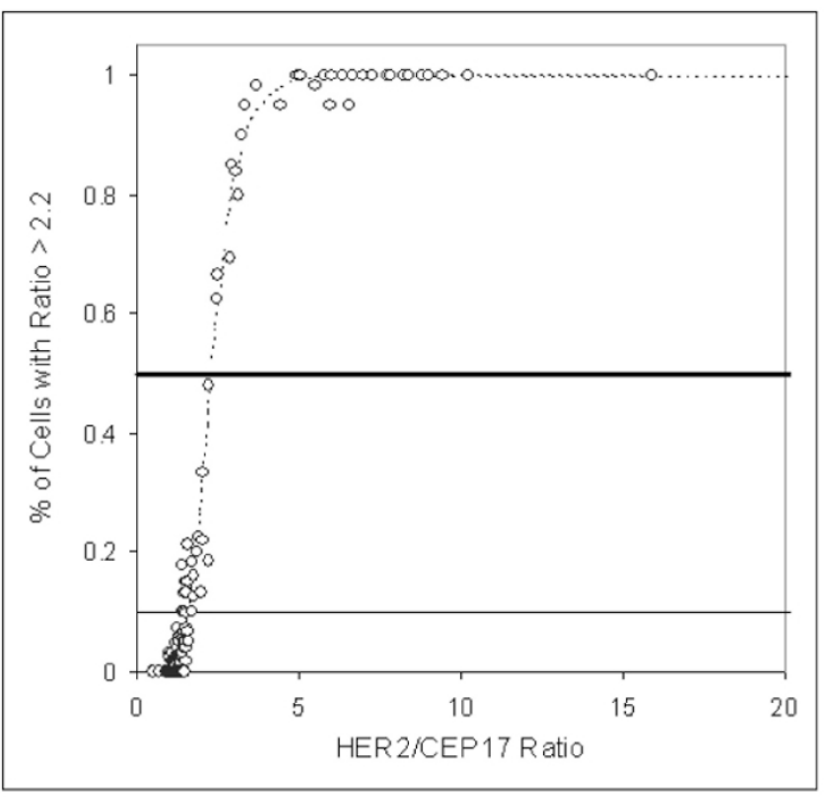

Figure 2: \%-cells HER2+ behaves as a function of HER2/CEP17 ratio. When fit to a standard (4-parameter) logistic curve, 50\%-cells HER2+ (thick horizontal line) corresponds to a HER2/CEP17 ratio of 2.2, whereas $10 \%$-cells HER2+ corresponds to a HER2/CEP17 ratio of 1.6 .

Conclusions: In keeping with the bulk of prior clinical trials, HER2/CEP17 ratio and HER2 copy number in the overall tumor are informative of HER2-positivity. The $\%$-cells positive behaves as a function of HER2/CEP17 ratio. Using 10\%-cells HER2 positive may overestimate the extent of HER2 positivity by lowering the threshold.

244 Expression of MET and PTEN in Triple Negative Breast Cancer $S$ Lau, S Rost, H Koeppen. Genentech, South San Francisco, CA.

Background: Triple negative breast cancer (TNBC) accounts for approximately $15 \%$ of ductal breast cancer and is characterized by an aggressive clinical course. Also, in contrast to hormone receptor (HR)- and HER2-positive breast cancer, targeted therapies are currently not available for TNBC patients. We determined MET and PTEN expression as relevant biomarkers for targeted therapies currently in clinical development for various indications. PTEN is being evaluated as predictive marker for therapies targeting the Pi3K/AKT pathway and MET-targeted therapies have recently shown early clinical promise in MET-positive lung and gastric cancer.

Design: Paraffin sections of 178 TNBC cases from patients enrolled in a global phase II trial testing efficacy of the anti-MET antibody onartuzumab in patients with metastatic TNBC were stained for MET and PTEN using antibodies SP44 (Ventana) and 138G6 (Cell Signaling Technology), respectively. Cases were scored as MET-High if 50\% or more tumor cells showed moderate or strong staining. PTEN expression was scored using an $\mathrm{H}$-score algorithm estimating \% tumor cells showing no, weak, moderate or strong staining. Cases with an $\mathrm{H}$-score of 0 were classified as PTEN-loss, cases with an $\mathrm{H}$-score between 1 and 100 were classified as PTEN-Low, cases with an H-score $>100$ were scored as PTEN-positive. The lack of expression of HR and HER 2 had been confirmed by the pathologist at the referring clinical site.

Results: $88 \%(156 / 178)$ of cases were classified as MET-Low; $45 \%$ of cases were negative ( $>50 \%$ of tumor cells without staining) and $43 \%$ were weakly positive ( $>50 \%$ of tumor cells weakly positive) for MET. $12 \%$ of cases were classified as MET-High with $21 / 22$ cases showing moderate and one case showing strong staining for MET in $>50 \%$ of tumor cells. $17 \%(29 / 166)$ of cases showed loss of PTEN and $15 \%$ showed decreased levels of PTEN (PTEN-low). There was no correlation between MET and PTEN expression levels.

Conclusions: Prevalence of PTEN loss appears lower in our series than previously published, which is possibly related to our stringent definition of PTEN loss. Moderate or high expression of MET was infrequently observed. The role of MET as therapy target in TNBC remains to be determined; updated efficacy results from the onartuzumab trial will be presented at the meeting.

PhH3 as a Potential Prognostic/Predictive Marker for Breast

LH Lee, G Bigras, $H$ Yang. University of Calgary, Calgary, AB, Canada; University of Alberta, Edmonton, AB, Canada.

Background: Phosphorylation of histone $\mathrm{H} 3$ is crucial for chromosome condensation and cell cycle progression during mitosis. Phosphohistone $\mathrm{H} 3(\mathrm{PhH} 3)$ can be visualized in dividing cells from late $\mathrm{G} 2$ phase to early telophase by immunohistochemical stain (IHC). The prognostic and predictive ability of $\mathrm{PhH} 3$ count in breast cancer is unknown. The objective of this study is to investigate the correlation of $\mathrm{PhH} 3$ count with known Oncotype DX recurrence score (RS), MBR grade, and mitotic score.

Design: Cases of invasive breast carcinoma with Oncotype DX RS results from our institution were selected with seven cases from each of high, intermediate, and low recurrence scores. Representative whole tumor sections were stained for $\mathrm{PhH} 3$ (rabbit polyclonal, 1/200 dilution, Cell Marque Corp.). $\mathrm{PhH} 3$ counts were reported 
as the average of two pathologists' independent count of 10 consecutive high power fields (HPF, 40X objective) in the region of highest staining concentration. Pearson's correlation coefficient between $\mathrm{PhH} 3$, RS, MBR grade, and mitotic score was calculated using IBM SPSS 19.

Results: Of the 21 cases identified, $\mathrm{PhH} 3$ correlated best with $\mathrm{RS}(\mathrm{r}=0.855, \mathrm{p}<0.001$, Figure 1). Other correlations are: $\mathrm{PhH} 3$ vs mitotic score $(\mathrm{r}=0.797, \mathrm{p}<0.001), \mathrm{PhH} 3$ vs MBR grade $(\mathrm{r}=0.736, \mathrm{p}<0.001)$, mitotic score vs $\mathrm{RS}(\mathrm{r}=0.832, \mathrm{p}<0.001)$, and MBR grade vs RS ( $(=0.791, p<0.001)$. Significant correlation between $\mathrm{PhH} 3$ and $\mathrm{RS}$ remained after controlling for mitotic score by partial correlation ( $\mathrm{r}=0.572, \mathrm{p}=0.008)$.

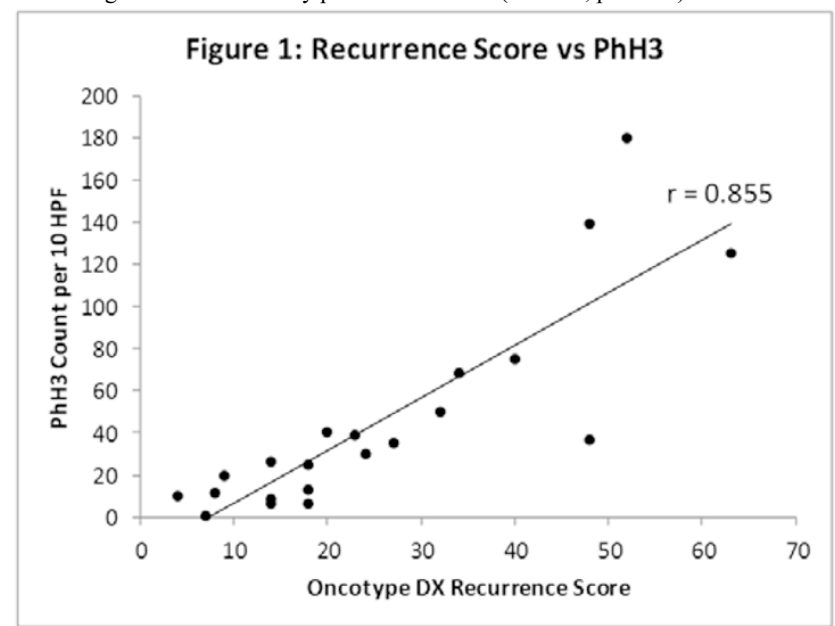

Conclusions: $\mathrm{PhH} 3$, RS, mitotic score, and MBR grade correlate well with each other. Correlation between $\mathrm{PhH} 3$ and RS is the strongest among the variables. Given that RS has an established strong relationship with prognosis and therapy responsiveness, $\mathrm{PhH} 3$ may also be an important predictive/prognostic measure in breast cancer. Also, the color contrast in $\mathrm{PhH} 3 \mathrm{IHC}$ is easily visualized and may help pathologists identify the areas with the highest mitotic activity, thus improving mitotic count accuracy for MBR grading. Future steps will be to increase case volume and compare $\mathrm{PhH} 3$ directly with patient outcome.

246

Invasive Paget Disease of the Breast: Twenty Years' of Experience at a Single Institution

$H$-W Lee, EY Cho. Samsung Medical Center, Sungkyunkwan University College of Medicine, Seoul, Republic of Korea.

Background: Mammary Paget's disease (MPD) is an uncommon intra-epidermal malignant neoplasm. MPD occupies approximately $1 \%$ to $3 \%$ of all primary breast cancers. MPD with dermal invasion (invMPD) is extremely rare, and its prognosis remains largely unknown. We reviewed MPD in our institution, and analyzed clinicopathological characteristics of invMPD and non-invMPD to compare their incidences and outcome.

Design: We conducted a retrospective review of 205 women with MPD diagnosed between 1994 and 2013 at Samsung Medical Center. We excluded some cases with direct invasion of underlying ductal carcinoma into dermis or epidermis. All slides and medical records were thoroughly reviewed. We investigated the clinicopathologic parameters, such as demographic data, intra-epidermal extent of MPD, underlying parenchymal disease data, and clinical outcome in both invMPD and non-invMPD. Mean follow-up period was 47.3 months.

Results: In all patients with MPD, 201 patients underwent excision and lymph node dissection and the remaining received excision only. Sixteen of 205 cases (7.8\%) of MPD showed dermal invasion of Paget cells. Out of 189 non-invMPD, 145 cases had underlying invasive ductal carcinoma, 41 DCIS, and 3 without underlying tumor. In 16 invMPD, 12 cases had underlying invasive ductal carcinoma, 3 DCIS, and 1 without underlying tumor. The mean depth and extent of Paget cell invasion in invMPD cases was $637.5 \mu \mathrm{m}$ and $1268.1 \mu \mathrm{m}$, respectively. The horizontal extent of MPD in invMPD was significantly larger (mean $14.31 \mathrm{~mm})$ than in non-invMPD $($ mean $7.35 \mathrm{~mm})(p=0.002)$. Distant metastasis and disease related death were observed in $12.7 \%(24 / 189)$ and $12.2 \%$ $(23 / 189)$ of patients with non-invMPD compared to $6.3 \%(1 / 16)$ with invMPD, and this difference was not significant $(p=0.7)$. Presence of invasion in MPD didn't correlate with mean size of underlying tumor $(p=0.592)$, lymph nodes metastasis $(p=1.00)$, estrogen receptor status $(p=0.41)$, HER2 expression $(p=1.00)$, treatment modality $(p=0.77)$, or poor prognosis $(p=0.7)$.

Conclusions: In this study, the extent of MPD was significantly correlated with presence of invasion in MPD. However, other clinicopathologic parameters were not associated with dermal invasion of MPD. In contrast to invasive extramammary Paget disease, dermal invasion in MPD was rare and didn't predict regional lymph nodes metastasis or poor prognosis. As most invMPD showed similar prognosis to non-invMPD, invMPD must be distinguished from locally advanced breast cancer presenting as satellite skin nodule.

247 Dual ERBB2 (HER2) Gene Protein Assay: Focused Study of Breast Cancers with 2+ Immunohistochemical Expression

$Z$ Li, DJ Dabbs, R Bhargava. Magee-Womens Hospital of UPMC, Pittsburgh, PA. Background: The combined gene protein assay (GPA) for HER2 (Ventana, Tucson $\mathrm{AZ}$ ) is a newly developed technique where both HER2 gene and protein can be simultaneously assessed on a single slide using bright field microscopy. The goal of the study was to compare this new GPA methodology with standard fluorescence in situ hybridization (FISH).

Design: This validation study included whole tissue sections of 50 invasive breast cancer cases with equivocal HER2 IHC results. For both FISH and GPA, HER2 amplification was defined as HER2/CEP17 ratio $\geq 2.0$. In addition, 25 HER2 IHC 3+ invasive carcinoma cases (on a tissue microarray, TMA) and 22 IHC negative (scores 0 and $1+$ on TMA) were also evaluated by GPA.

Results: GPA was successful in all cases in the first attempt. All previously tested IHC $2+$ cases were again scored as 2+ on GPA. The overall agreement between FISH and GPA with respect to HER2 gene status was $94 \%$.

Gene protein assay versus FISH for IHC $2+$ cases

\begin{tabular}{|c|c|c|c|}
\hline & FISH Amplified & FISH Not Amplified & Total \\
\hline GPA Amplified & 3 & 3 & 6 \\
\hline GPA Not Amplified & $=5$ & 44 & 444 \\
\hline Total & |3 & 47 & $\sqrt{50}$ \\
\hline
\end{tabular}

The 3 discordant cases were amplified by GPA and not amplified by FISH. The HER 2 to CEP17 ratio on these 3 cases by GPA was 2.0, 2.16, and 2.34 with average HER 2 copies/ cell of 5.7, 7.8, and 5.5 respectively. For the $50 \mathrm{IHC} 2+$ cases, statistical averages were also calculated. The mean $H E R 2$ copies per cell for FISH were 3.11 (sd: 1.81; range 1.83 to 11.07 ) versus 3.51 (sd: 1.84 ; range: 1.75 to 9.95 ) for GPA. The mean CEP17 signals per cell were 2.21 (sd: 0.61 ; range: 1.31 to 4.2 ) for FISH versus 2.30 (sd: 0.61 ; range: 1.20 to 3.85) for GPA. The mean for HER2:CEP17 ratio was 1.39 (sd: 0.66 ; range; 1.03 to 4.32 ) by FISH and 1.49 (sd: 0.65 ; range: 1.02 to 4.72 ) by GPA. All $25(100 \%)$ of IHC 3+ cases showed clusters of HER2 gene consistent with HER2 amplification by GPA. These 25 cases also showed strong membranous reactivity consistent with $3+$ IHC score. Similarly, the 22 known IHC negative cases on the TMA, were again IHC negative, and lacked HER2 gene amplification by GPA.

Conclusions: The HER2 GPA shows excellent concordance with both HER2 IHC assay and FISH assay. It performs accurately not only for unequivocally positive and negative cases but also on the most challenging (IHC 2+) breast cancer cases. This new assay combines the advantages of both IHC and FISH, i.e. accuracy similar to FISH for IHC equivocal cases, faster turnaround time (less than 24 hours) like IHC and evaluation under bright-field microscopy where the tissue morphology is well preserved and heterogeneity is better appreciated.

$248 \quad$ Nuclear Nano-Morphology Markers Predict Breast Cancer Risk in Patients with Atypical Breast Epithelial Hyperplasia

$Z$ Li, R Bhargava, $K$ Staton, $H$ Wang, S Uttam, RE Brand, A Soran, GM Ahrendt, $Y$ Liu. Magee-Womens Hospital of UPMC, Pittsburgh, PA; University of Pittsburgh, Pittsburgh, PA.

Background: Patients with atypical breast epithelial hyperplasia, i.e., atypical ductal hyperplasia (ADH) and lobular neoplasia (LN) are at increased risk for developing breast cancer. We recently developed a novel optical microscope, spatial-domain lowcoherence quantitative phase microscopy (SL-QPM) to analyze nanoscale structural characteristics of nuclei (i.e. nuclear nano-morphology markers) on histological slides. In this study, we aim to assess nuclear nano-morphology markers on predicting breast cancer risk in patients with $\mathrm{ADH} / \mathrm{LN}$.

Design: 78 patients with ADH/LN treated by excision with no more severe lesions were included in this study. 39 patients including $25 \mathrm{ADH}$ and $14 \mathrm{LN}$ (high risk group) developed more severe lesions (ductal carcinoma in situ or invasive cancer) during an up to 12 years follow-up period; the other 39 patients including $22 \mathrm{ADH}$ and $17 \mathrm{LN}$ (low-risk group) did not. The SL-QPM analysis was performed on $\sim 50-60$ nuclei of $\mathrm{ADH} / \mathrm{LN}$ cells from histological slides. The univariate Cox models were used to evaluate nuclear nano-morphology markers' effectiveness on predicting breast cancer risk.

Results: We identified 4 markers ( $\angle \mathrm{OPD}>$ : a measure of nuclear density; skewness: a measure of nano-structural distribution; <amp $>$ and $\sigma_{\text {amp }}$ : measures of variation of refractive index profile) which showed significant difference between these two groups (p-value $<0.05$ ). Furthermore, the increased hazard ratio after combining any two makers demonstrated additive effect on predicting breast cancer risk.

Nuclear nano-morphology markers predict breast cancer risk in patients with $\mathrm{ADH} / \mathrm{LN}$

\begin{tabular}{|c|c|c|c|}
\hline ical marker & p value & Hazard ratio & $95 \%$ confidence interval \\
\hline COPD $>$ & 0.0274 & 2.70 & $1.12-6.52$ \\
\hline Skewness & 0.0135 & 2.51 & $1.21-5.21$ \\
\hline amp> & 0.0026 & 2.74 & $1.42-5.28$ \\
\hline бamp & 0.0135 & 2.32 & $1.19-4.54$ \\
\hline$\langle\mathrm{OPD}>*<\mathrm{amp}>$ & 0.0005 & 3.21 & $1.66-6.22$ \\
\hline KOPD $>*$ бamp & 0.0016 & $\sqrt{3.01}$ & $1.52-5.95$ \\
\hline$\overline{\text { <amp }>*^{*} \sigma a m p}$ & 0.0007 & 3.43 & $168-6.97$ \\
\hline
\end{tabular}

No statistically significant difference in patient age was observed between low- and high-risk patients.

Conclusions: This is the first clinical study to demonstrate the feasibility of using nuclear nano-morphology markers to predict breast cancer risk in patients with ADH/ $\mathrm{LN}$. This technique might be used as a cost-effective test to assess breast cancer risk in patients with $\mathrm{ADH} / \mathrm{LN}$ clinically.

249 Triple Negative Breast Cancers Lack Nuclear FOX1 Expression $X$ Li, GH Smith, FE Castaneda-Valladares, MJ Giri, GM Oprea-Ilies, C Cohen. Emory University, Atlanta, GA; ISSEMY-Tlalnepantla, Tlalnepantla, Mexico; Georgia Regions University, Augusta, GA.

Background: Forkhead-box (FOX) family proteins are DNA binding proteins regulating transcription and DNA repair. FOX1 functions as tissue specific alternative splicing regulator, stimulates expression of genes involved in cell division, attenuation of oxidative stress, tumor metastasis, including epithelial-mesenchymal transition (EMT), cell migration, and drug resistance. The recurring association of Fox binding 
site (UGCAUG) with tissue-specific alternative exons suggests an important alternative splicing regualtor role. FOX1 depletion causes cell death by mitotic catastrophe, occuring during mitosis, oftern due to aberrant G2 checkpoint control. Therefore FOX1 inhibiton could represent a potential theranostic biomarker in breast cancer. The aim of the study was to assess FOX1 immunohistochemical (IHC) expression in subtypes of breast cancer in correlation with clincal presentation and outcome.

Design: Invasive mammary carcinomas (IMC) were grouped in hormone receptor positive (HRP) and triple negative tumors (TNT). The HRP carcinomas included estrogen and/or progesterone receptor positive tumors. The TNTs included tumors that were hormone receptor and did not overexpress HER2. Tissue microarrays (TMAs) were constructed with two $1 \mathrm{~mm}$ representative cores from each IMC and were stained with FOX1 monoclonal antibody. Nuclear staining in more than $5 \%$ of cells was considered positive. These data along with age, race: African-American (AA) and Caucasians (CS), tumor grade and stage were entered in an excel spreadsheet.

Results: A total of 206 breast cancer patients were evaluated: 87 had TNT and 119 had HRP tumors. The age at diagnosis ranged from 24-89 years. FOX1 expression was statistically significant associated with HRP breast cancers $(p=0.0001)$ while only 13 TNT expressed FOX1. In the TNT group, AA tended to show less FOX1 expression. The age, tumor grade and stage and outcome did not show statically significant differences. Conclusions: 1. Nuclear expresion depletion of FOX1 may differentiate breast cancer tumor subtype and explain the more aggressive nature of TNT. Furthemore it may be associated with carcinogenisis and tumor progession in AA.

2. Targeted inhibition of FOX1 may represent a powerful theranostic agent in breast cancer management.

250 Prognostic Significance of Multifocal Lesion in ER+ Breast Cancers $Y$ Liang, X Li, J Wang, L Huo, C Albarracin, Y Gong, Y Wu. The University of Texas MD Anderson Cancer Center, Houston, TX; Emory University, Atlanta, GA; General Hospital, Jinan Command of the People's Liberation Army, Jinan, China.

Background: Multifocality is relatively common in breast cancers. However, whether it is an adverse prognostic factor remains controversial. Most published studies on the subject focus on clinicopathologic features without emphasis on the intrinsic biological characteristics of the tumor, including ER, PR and Her2.

Design: We retrospectively reviewed 812 consecutive primary surgical resections of breast cancer without pre-operative neoadjuvant hormonal or chemotherapy from 2002 -2004 in our institution. We identified 119 cases with more than 1 focus of invasive carcinoma, considered as multifocal breast cancer (MFBC). Demographic, pathologic, biological and clinical outcomes were compared between MFBC and unifocal breast cancer (UFBC).

Results: The incidence of synchronous ipsilateral MFBC in the current study is $15 \%$. MFBC occurs more in white women $(82 \%)$ compared to UFBC $(78 \%)(p=0.024)$ MFBC is often associated with larger tumor size (measured from the dominant tumor) $(17.3 \pm 9.5 \mathrm{~mm})$ compared with UFBC $(16.9 \pm 12.1 \mathrm{~mm})(\mathrm{p}=0.001)$, more frequent regional lymph nodal metastasis $(\mathrm{p}=0.004)$, and high pathologic stage $(\mathrm{p}=0.015)$ There are no differences in ER, PR, HER2 status and nuclear grade between MFBC and UFBC groups. However, with mean follow-up time of 102 months, ER+ MFBC showed significant worse disease free survival (DFS) compared to ER+ UFBC (logrank test $\mathrm{p}=0.022$ ) (Figure 1 ). No prognostic difference was seen in other subtypes including HER2+ and triple negative groups, probably due to small case number in the MFBC group.

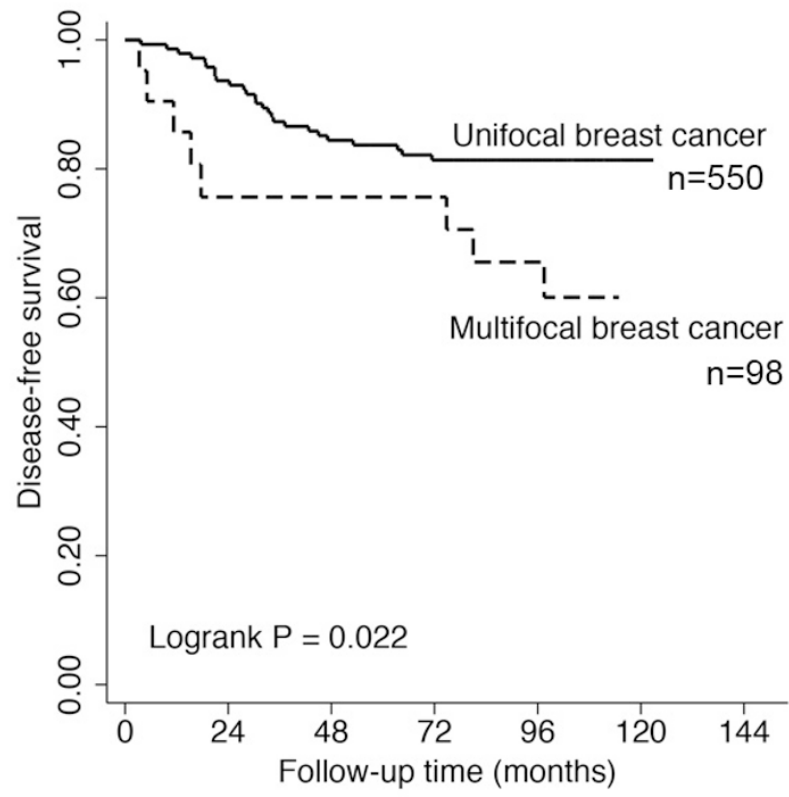

Conclusions: Amongst ER + patients, the present of multifocal lesion is associated with a worse DFS. Due to small number of HER2+ and triple negative cases in the MFBC group, additional study with larger number of cases may need to clarify whether HER2+ and triple negative MFBC also carry worse prognosis than those with UFBC.
251 Evaluation of C-Myc Expression by Immunohistochemistry (IHC) in Invasive Breast Carcinoma (BC)

G Liles, S Mohanty, F Chung, S Alkan, F Dadmanesh. Cedars-Sinai, Los Angeles, CA. Background: The c-myc oncoprotein is a transcription factor that regulates various target genes encoding proteins that initiate carcinogenesis and maintain the cells in a transformed state. Recent investigations have shown that triple negative (TN) BCs have increased c-myc expression due to altered expression of c-myc regulatory genes, resulting in increased activity of the c-myc pathway. In addition, these tumors are suggested to be responsive to specific therapies such as targeted cyclin-dependent kinase inhibition. To date, no studies have investigated expression of c-myc in these tumors using immunohistochemistry (IHC). Therefore, we sought to study c-myc expression by IHC, not only in TN BC but also in other molecular subtypes.

Design: We selected 88 cases of invasive $\mathrm{BC}$ from our pathology database: $53 \mathrm{TN}$, 17 Luminal A, 9 Luminal B, and 9 Her-2neu subtypes. IHC detection of c-myc was performed (Ventana Medical Systems) and nuclear staining was evaluated using a 4 point scale (0-3) and percent positivity. Distribution across molecular subtypes was tested using chi-squared analysis.

Results: Positivity for c-myc expression was significantly different across molecular subtypes (p-value $<0.0001$ ) with $92 \%$ in $\mathrm{TN}, 41 \%$ in Luminal A, $33 \%$ in Luminal B, and $56 \%$ in Her-2neu (Figure 1). All the positive cases show moderate to strong ( 2 to $3+)$ nuclear staining in greater than $10 \%$ of tumor cells.

Figure 1. C-myc Expression by IHC

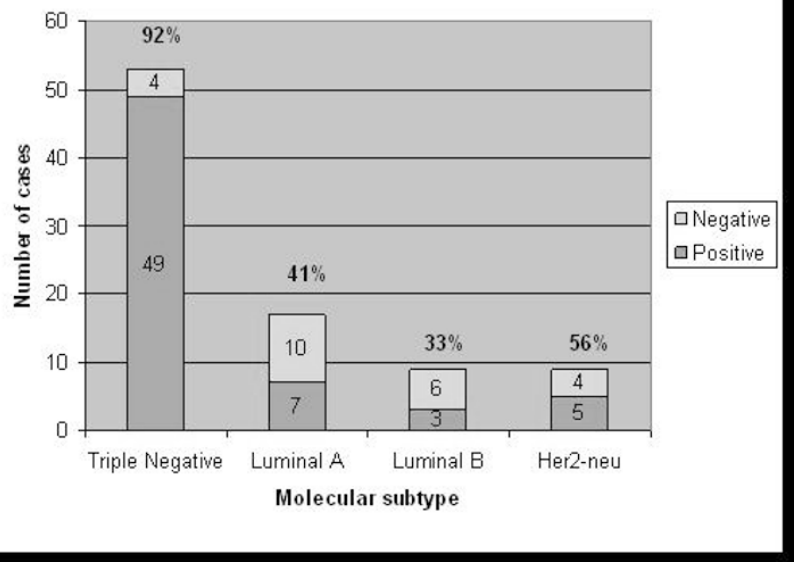

Conclusions: C-myc expression by IHC in TN BCs correlates with previous genomic and molecular data suggesting increased activity of the myc signaling pathway. Therefore, newer therapeutic modalities targeting c-myc for treatment of TN BC are warranted. If these targeted therapies become available in the future, c-myc IHC may potentially play an important role in predicting therapy response.

\section{Lobular Intraepithelial Lesions on Core Biopsies: To Excise or Not} to Excise?

CA Llanos, S Umar, M Jorda, C Gomez-Fernandez. University of Miami, Miami, FL. Background: The significance of lobular intraepithelial lesions encompassing atypical lobular hyperplasia (ALH), low grade or classic lobular intraepithelial neoplasia (LGLIN), and high grade lobular intraepithelial neoplasia (HG-LIN), remains uncertain. Consequently, the management of these conditions when diagnosed on core biopsy is not well established. The aim of this study was to evaluate our experience in the follow-up for patients diagnosed with these entities.

Design: We reviewed core biopsies diagnosed with ALH, LG-LIN, and HG-LIN, and the subsequent surgical excisional specimens whenever available. Diagnostic criteria were as follows: ALH: loosely cohesive cellular proliferation partially or completely filling the ductules without distension; LG-LIN: loosely cohesive cellular proliferation completely filling and distending at least some of the ductules; HG-LIN: loosely cohesive cellular proliferation filling and maximally distending the ductules (macroacinar), and/ or increased nuclear atypia (pleomorphic), signet ring cell features, presence of necrosis. IHC for E-cadherin was negative in all cases included in the study.

Results: There were a total of 44 core biopsies (ALH (24), LG-LIN (11), HG-LIN (9). Of the 24 patients with ALH, 17 had subsequent surgical excision, including $2(12 \%)$ patients initially diagnosed with concurrent invasive carcinoma. The remaining 15 patients had benign disease on subsequent excision. Of the 11 patients with LG-LIN on core biopsy, 10 had subsequent surgical excision and out of this patients, $8(80 \%)$ had invasive carcinomas. 7 with concurrent invasive lobular carcinoma (ILC) and one, with DCIS, had invasive ductal carcinoma (IDC) on surgical excision. For the 9 patients with HG-LIN, all had follow-up surgical excision. 5 patients had concurrent invasive carcinoma on initial core biopsy (4 ILC, 1 IDC). Subsequent surgery identified an additional 2 ILC for a total of 7 patients (78\%) with invasive carcinoma.

Conclusions: The majority of patients with ALH on core biopsies were pure and not associated with other pathologic findings. In the absence of any other pathologic findings, patients with ALH who undergo short term surgical excision have benign disease. In contrast, core biopsies with LIN2 and LIN3 are more likely to be associated with ipsilateral invasive carcinoma at initial core biopsy diagnosis and at subsequent 
resection. This suggests that patients with both LG-LIN and HG-LIN should generally be considered for surgical excision to rule out a more significant lesion, while patients with pure ALH may benefit from a more conservative approach.

253 Atypical Lobular Hyperplasia, Are We Treating Too Soon?

CA Llanos, S Umar, M Jorda, C Gomez-Fernandez. University of Miami, Miami, FL. Background: Clinical significance and long term prognosis of atypical lobular hyperplasia (ALH) is not completely understood. Therefore, subsequent management of this condition does not have a well established approach, and surgical resection is frequently the preferred treatment. The aim of this study was to evaluate the findings of the patients who underwent resection after the diagnosis of ALH was made.

Design: A retrospective search of laboratory and medical records was performed for ALH diagnosis in biopsy specimens. Diagnostic criteria for ALH was defined as a loosely cohesive cellular proliferation partially or completely filling the ductules without distension. We retrieved the concomitant resections specimens if available. Evaluation of the associated pathologic findings present at the time at the biopsy and the resection was performed and compared among cases as well as the time interval between the biopsy and resection.

Results: There were a total of 22 core biopsies identified. $15(68 \%)$ of the cases were managed surgically while 7 (32\%) of the cases were given a conservative management. The mean time interval between biopsy and surgical resection was 2.2 months. No additional abnormality was identified in the patients that underwent conservative management. In the patients that underwent surgical management, none of the biopsy specimens had an associated pathology other than benign changes and in the concurrent resection specimens, no malignant or premalignant lesions were identified despite extensive sampling.

Conclusions: The majority of patients with ALH on core biopsies received short term surgical excision despite the lack of any other pathologic findings at the time of biopsy. Moreover, surgical specimens from these patients failed to reveal a more ominous lesion. The short interval ( 2.2 months) between biopsy and surgical management and the absence of additional lesions at the time of resection suggest that a different and more conservative approach for this lesion may be ultimately more beneficial for patients.

\section{Claudin 4 and 7 Expression Is Not Correlated to Triple Negative Phenotype in Invasive NOS Breast Carcinomas: A Wide Series Assessed} by Immunohistochemistry

AF Logullo, MA Stiepcich, S Nonogaki, R Malagoli, MM Brentani, FS Pasini, FA Soares. UNIFESP-EPM, São Paulo, Brazil; FLEURY, São Paulo, Brazil; A.C. Camargo Cancer Center, São Paulo, Brazil; FMUSP-Brazil, São Paulo, Brazil.

Background: Molecular phenotype has improved the understanding of wide range of different subsets of breast cancer disease. Recently, a new molecular subtype denominated "claudin low" was described among the metaplastic triple negative cases, and associated to worse prognosis and CD24/44 stem cell profile. Among more than 19 proteins called claudins, isotype 4 and 7 are the most common in breast tissue and breast carcinomas. Thus, we aimed to verify the differences in claudin immunoexpression among Luminal A, Her-2, and triple negative phenotypes; and CD24/44 status.

Design: We evaluated estrogen and progesterone receptor status (ER,PR) HER-2 expression, cytokeratin 5/6 and CD24/44 profiles in 745 invasive breast carcinomas SOE disposed in a Tissue Micro Array and correlated to Claudin 4 and 7 expression determined by immunohistochemistry. ER and PR were assessed by Allred's method. HER2 expression was evaluated by ASCO/CAP guidelines, as well Cytokeratin 5/6, claudin 4 and 7 staining. CD24/44 was considered positive when more than $10 \%$ of cells were dark brown.

Results: 486 cases were positive for claudin 4 and 340 cases for claudin 7 . The majority of claudin 4 negative cases were clustered in luminal A cases $-185(70.6 \%)$ whereas only $57(21.8 \%)$ showed triple negative profile. The remaining 20 cases $(7.6 \%)$ exhibited strong HER-2 expression $(\mathrm{p}=0.001)$. The same trend was observed for the lack of claudin 7 expression: 205 cases $(61.5 \%)$ were Luminal A, 50 cases $(15.0 \%)$ were HER-2 positive, and the remaining 79 cases $(23.6 \%)$ were triple negative. When both claudins 4 and 7 expression were negative, the majority of the cases were luminal A [82 (70.7\%)], instead of triple negative cases [27 $(23.3 \%)]$ or HER-2 expressing cases [7 $(6.0 \%)]$. Claudin 4 expression clearly correlated to ER, PR and HER-2 expression $(p=0,01)$. On the other hand, claudin 7 corelated to CD24/44 profile $(\mathrm{p}=0,04)$.

Conclusions: Claudin expression. profile, -namely claudin low- assessed on protein level by immunohistochemistry, does not reflect the molecular clustering in molecular subtype triple negative profile. However, claudin 4 and 7 show distinct correlation to stem cell markers; being claudin 7 correlated to CD24/44 profile.

255

Is p53 an Independent Marker of Aggressiveness in an Ethnically Diverse Sample of Breast Cancer Patients, after Accounting for Molecular Subtype?

AM Mahmoud, V Macias, JK Kresovich, KA Dookeran, A Kajdacsy-Balla, EL Wiley, GH Rauscher. University of Illinois at Chicago, Chicago, IL

Background: Immunohistochemical (IHC) p53 expression has been demonstrated to be a prognostic marker in invasive breast cancer. The goal of these analyses was to examine the extent to which p53 expression was associated with specific breast cancer subtypes, and whether it could serve as an additional prognostic marker above and beyond breast cancer subtype, in an ethnically diverse sample of 287 patients ( 86 Non-Hispanic White, 84 Hispanic and 116 African American).

Design: IHC analysis was performed on tissue microarrays constructed from invasive breast cancer samples obtained from the Breast Cancer Care in Chicago study (patients diagnosed between 2005-2008). Tissue samples were tested for the expression of estrogen receptor (ER), progesterone receptor (PR), HER2, EGFR and CK5/6. From these results, breast cancers were classified as Luminal A ( $\left.\mathrm{ER}^{+} / \mathrm{PR}^{+} / \mathrm{HER} 2^{-}\right)$, Luminal $\mathrm{B}\left(\mathrm{ER}^{+} / \mathrm{PR}^{+} / \mathrm{HER} 2^{+}\right), \mathrm{HER} 2$ enriched $\left(\mathrm{ER}^{-} / \mathrm{PR}^{-} / \mathrm{HER}^{+}\right)$, and triple negative (TN) (ER $/$ $\left.\mathrm{PR}^{-/ H E R} 2^{-}\right)$. TN tumors were subclassified into basal like (BL) if they expressed either EGFR or CK5/6 or otherwise classified as unspecified (US) if negative for both EGFR and CK5/6. P53 expression was classified as low $(<20 \%)$ or high $(\geq 20 \%)$. We used multivariable logistic regression to examine associations between p53 expression, breast cancer subtype, and histological grade, controlling for race/ethnicity and age at diagnosis in all models.

Results: In adjusted models, the proportion of tumors with a high p53 expression was much lower for luminal A(13\%) than for luminal B, HER2, TN-US, and TN-BL subtypes $(38,30,57$ and $59 \%$, respectively, $\mathrm{p}<0.0001)$. The proportion of tumors that have high histologic grade was also much lower for luminal A $(29 \%)$ than for luminal B, HER2, TN-US, and TN-BL subtypes $(85,86,94$ and $68 \%$, respectively, $\mathrm{p}<0.0001)$. Before accounting for subtype, $68 \%$ of high p 53 tumors were high grade, compared with only $37 \%$ of low p53 tumors ( $<0.0001)$; after controlling for breast cancer subtype these percentages were $55 \%$ and $42 \%$, respectively, $\mathrm{p}=0.09$ ).

Conclusions: In this multi-ethnic sample of breast cancer patients, we found that p53 might serve as an independent predictor of more aggressive, higher grade disease, after accounting for molecular subtype.

\section{Determining HER2 Gene Amplification Status in Technically} Unsatisfactory Cases

A Maskovyak, CLanigan, E Downs-Kelly, RR Tubbs. Cleveland Clinic, Cleveland, $\mathrm{OH}$. Background: Accurate assessment of ERBB2 (HER2) in breast cancer is critical in providing prognostic information and identifying patients who may benefit from trastuzumab. HER 2 testing by fluorescence in situ hybridization (FISH) is sensitive and specific, but is subject to pre-analytical variables such as fixation duration and medium or specimen quality. At times, these variables can result in a technically unsatisfactory (tech unsat) assay which is uninformative. INFORM HER2 DNA Probe Cocktail assay (HER2 Dual ISH)(Ventana Medical Systems Inc., Tucson, AZ) is a bright field in situ hybridization assay that has been shown to be robust; the focus of this study was to investigate HER2 Dual ISH as a rescue assay in HER2 FISH tech unsat cases.

Design: HER2 FISH tech unsat cases were identified from 01/2010 through 06/2012. A 4 micron fixed, paraffin-embedded tissue section that was cut at the time of the original FISH assay was subjected to HER2 Dual ISH on the BenchMark XT automated instrument. Cases were evaluated according to the package insert with HER2/CHR17 ratios recorded. When available, results of HER2 Dual ISH were compared to 4B5 immunohistochemistry (IHC; Ventana Medical Systems Inc.) or HER2 FISH (Abbott Molecular Vysis) performed on the same tissue or an alternative specimen from the same tumor.

Results: There were 3812 HER FISH assays performed from 01/2010 through 06/2012; 111 cases of either a breast primary or a metastasis from 105 patients were tech unsat. Fixatives included zinc formalin, alcoholic formalin, CytoLyt, neutral buffered formalin, and decal. Of 111 cases, 92 had tissue available for Dual ISH; $32(35 \%)$ had scorable signals which allowed for HER 2 genotyping ( 30 non amplified, 2 amplified). Two cases had scorable signal but no residual invasive carcinoma, 17 cases had CHR 17 signal but no scorable HER 2 signal, and 42 cases had weak endogenous signal not amendable to scoring or no signal. HER2 status on the same tissue (determined by IHC, $n=14$ ) or an alternative specimen from the same tumor (determined by FISH, $\mathrm{n}=11$ ) were available in 25 of the 32 cases genotyped by Dual ISH with excellent concordance ( 2 amplified, 3 equivocal, and 20 non amplified). Of the 3 cases that were equivocal by IHC, one had historical HER2 FISH which was non-amplified and no additional information was available on the other two cases.

Conclusions: HER 2 Dual ISH appears to be a robust assay with excellent concordance with other testing methodologies and the ability to overcome some of the pre-analytical variables that pose a problem in HER2 assessment by FISH.

257 Carcinoma

SMasood, E El-Gabry, C Zhang, B Garrison, R Bertholf, Z Wang. University of Florida College of Medicine-Jacksonville, Jacksonville, FL.

Background: Telomerase reactivation is a relatively early event in mammary tumorigenesis. DNA methylation at a $\mathrm{CpG}$ island rich region $\sim 600 \mathrm{bp}$ upstream the transcription start site was documented to play an important, yet complicated, role in $h T E R T$ gene regulation. This study further investigates the DNA methylation on surgical specimens using pyrosequencing. One such endeavor leads to establishment of a potential methylation index that helps distinguish breast malignancies from normal and thus highlights its significance in clinical diagnostics.

Design: The study includes 87 cases with invasive ductal carcinoma. DNA of tumor and normal breast was isolated (Gentra ${ }^{\circledR}$ Puregene). bisulfite-modified (SYMO RESEARCH), and PCR amplified. Pyrosequencing was performed to determine methylation levels of $10 \mathrm{CpG}$ islands: four "upstream" (-634 bp -655 bp) and six "downstream" (-548 bp -581 bp). Methylation was divided into three levels: upregulated $(>50 \%)$, intermediate $(30 \% \sim 50 \%)$ and down-regulated $(<30 \%)$. Equivocal cases with down-regulation in tumor or above down-regulation in normal breast were repeated on microdissected tissue from formalin fixed paraffin embedded (FFPE) sections (TrimGen Inc.). The data was analyzed by chi-square test for statistical significance.

Results: A significant global increase of methylation was noted in tumors at all of the $10 \mathrm{CpG}$ islands $(\mathrm{p}<0.01)$. Differential methylation was best seen in the first $\mathrm{CpG}$ island of the upstream region where 78 of $87(90 \%)$ tumors showed greater than intermediate level methylation (up-regulated:intermediate $=57: 21)$; 81 of $87(93 \%)$ normal breast displayed down-regulation with none being up-regulated. The six downstream $\mathrm{CpG}$ islands exhibited no upregulation and two intermediate methylation in normal breast. 
Further data culling revealed that of the nine tumors with down-regulation in the upstream region, six were status post neoadjuvant therapy; one had no residual tumor; two were due to paucity of invasive tumor. Repeated assays on microdissected FFPE sections enhanced the data.

Conclusions: Methylation of $h T E R T$ gene may be further explored and developed as an index for breast cancer diagnostics in a larger case cohort. Loss of methylation in tumors status post chemotherapy could provide 1) a rare molecular marker to determine the recurrent essence vs. metachronous nature of a new tumor; 2) research opportunities on mechanisms other than methyaltion utilized by recurrent tumor. Expansion of the study to include pre-malignancies and different histologic types of breast cancer appears justified.

258 Incidental Radial Scars in Needle Core Biopsy Have a Lower Frequency of Pathologic Upgrade at Excisional Biopsy Than Other Types of Radial Scars

C Matrai, L Pharmer, MB Drotman, TM D'Alfonso, SJ Shin. Weill Cornell Medical College, New York, NY.

Background: Radial scar (RS) is a benign lesion that may be detected on imaging as a mass, architectural distortion, or as calcifications. Currently, patients with RS diagnosed on NCB undergo excisional biopsy (EXBX) due to the concern that a more significant lesion (pathologic upgrade) associated with the RS had gone unsampled. It is unclear whether radiographically-targeted RSs show the same frequency of pathologic upgrade as those which are incidental. We hypothesize that incidental RSs show a lower frequency of pathologic upgrade than non-incidental ones which is the focus of this study.

Design: 75 patients with RS diagnosed on NCB with subsequent EXBX were identified. Clinical and radiographic data were reviewed. Slides were reviewed and microscopic features were recorded including size of RS, number of involved cores, presence and location of calcifications, and completeness of excision of RS in the NCB. Cases with coexisting findings that warrant EXBX [e.g. atypical duct hyperplasia (ADH)] were excluded. RSs were categorized as targeted or incidental after histologic and radiographic correlation. Cases were deemed indeterminate if it was uncertain whether the RS represented the targeted lesion (e.g. NCB performed for MRI enhancement or targeted calcifications present in both the RS and adjacent tissue). Pathologic upgrade was defined as the presence of atypia or carcinoma in the EXBX.

Results: Pathologic upgrade was seen in $9 / 75$ cases $(12 \%)$. Size of the RS or completeness of excision was not significantly different among RSs that were radiographic targets, incidental, or indeterminate. Of the 15 radiographically-targeted RSs, the pathologic upgrade rate was $20 \%(3 / 15 ; 2 \mathrm{ADH}, 1 \mathrm{ALH})$. Of the 13 incidental RSs, the pathologic upgrade rate was $8 \%(1 / 13 ; 1$ LCIS $)$. Among 47 RSs of indeterminate nature, the pathologic upgrade rate was $11 \%(5 / 47 ; 5$ LCIS). $2 / 9$ cases with pathologic upgrade showed residual RS in the EXBX ( 1 targeted, 1 indeterminate). Of the 9 cases with pathologic upgrade, the upgraded lesion was adjacent to the biopsy site in 4 (all indeterminate) of which 1 also had residual RS in the EXBX. The remaining 5 were distant from the biopsy site with or without residual RS.

Conclusions: Radiographically targeted RSs and incidental RSs found in NCB demonstrate divergent pathologic upgrade rates in subsequent EXBXs. Acknowledging inherent selection bias in our study, the true pathologic upgrade rate for incidental RSs is likely even lower, leading us to consider that such patients may be candidates for non-surgical management at the time of NCB.

259 The Association of Sentinel/Axillary Lymph Node Metastasis with Lymph Node Reactive Patterns and FOXP-3 Positive T Regulatory Cells in Breast Cancer

SH Mauzo, H Sun, M Li, RL Hunter, S Zhang. University of Texas Health Science Center at Houston, Houston, TX.

Background: Early studies showed that sinus histiocytosis was associated with marked prolonged survival and germinal center hyperplasia in axillary lymph nodes was associated with cancer metastasis. Recently, some studies have shown that FOXP-3 regulatory $\mathrm{T}$ cells were associated with lymph node metastasis and prognosis in breast cancer, gastric cancer and melanoma. The goal of this study is to correlate the lymph node reactive patterns, FOXP-3 cell count and nodal metastatic status.

Design: Cases of breast cancer with sentinel nodal biopsy or axillary lymph node dissection were included in the study. The lymph node reactive patterns were reviewed by a breast pathologist, and FOXP-3 immunohistochemistry was performed on cases with available material. The FOXP-3 positive cells were blindly counted in three areas under high power field using light microscope and the mean was used. Fisher exact test and student t-test were used for statistic analysis.

Results: 47 cases were reviewed and 2 cases were excluded from the study due to total replacement of lymph node structure with metastasis. 45 cases included 37 invasive ductal carcinoma, 6 invasive lobular carcinoma, 1 metaplastic carcinoma and 1 ductal carcinoma in situ. There were 18 cases with lymph node metastasis including 3 micrometastasis and 27 negative for metastasis. Germinal center hyperplasia was noted in 25 cases and other reactive patterns (sinus histiocytosis and interfollicular hyperplasia) were in the remaining 20 cases. Lymph node metastasis was present in $17 / 25$ patients with germinal center hyperplasia and only in $1 / 20$ patients with other reactive patterns $(\mathrm{p}=0.001)$. FOXP-3 positive regulatory $\mathrm{T}$ cell counts showed no significant differences between the positive and negative lymph nodes $(272 \pm 88 / \mathrm{HPF}$ vs $345 \pm 198 / \mathrm{HPF}, \mathrm{p}=0.34$ ), or between lymph nodes with germinal center hyperplasia or other reactive patterns $(309 \pm 146 / \mathrm{HPF}$ vs $334 \pm 209$, $\mathrm{p}=0.74)$.

Conclusions: Germinal center hyperplasia is significantly associated with sentinel/ axillary lymph node metastasis in breast cancer. There is no significant difference in FOXP-3 positive $\mathrm{T}$ regulatory cells among all groups. Future study will explore the relation between lymph node reactive patterns and tumor cell immunity.
GATA3 Has Prognostic Value in Advanced Breast Cance

BC McCleskey, T Nguyen, WE Grizzle, K Zhang, O Hameed, GP Siegal, S Wei. University of Alabama at Birmingham, Birmingham, AL.

Background: GATA3 is a transcription factor regulating luminal cell differentiation and has been implicated in the luminal A type of breast cancer (BC). Its expression is maintained in metastatic BCs showing loss of ER/PR. Further, the molecule is reportedly expressed in a subset of triple negative BCs (TNBCs). There have been conflicting findings on the utility of GATA3 expression for BC prognosis. The aim of this study was to explore the relationship between GATA3 expression and established clinicopathologic features and to evaluate its prognostic significance in a subset of patients with advanced $\mathrm{BCs}$

Design: Tissue microarrays were constructed from 62 primary BC cases with known distant (bone/viscera/brain) metastases obtained between 1997-2004 and 10 control BC cases (no metastases with at least 8 years of follow up). Nuclear immunoexpression of GATA3 was assessed by multiplying the intensity (0-3) and \% tumor cell staining to determine a $\mathrm{H}$-score. $\mathrm{X}^{2}$ test and Cox Proportional Hazard Model were used for statistical analyses.

Results: GATA3 expression was significantly higher in BCs with distant metastases than controls $(\mathrm{p}=0.04)$. Among advanced $\mathrm{BCs}$, its expression was not associated with age at diagnosis, race, tumor type, size, nodal status, or specific organ relapse. As expected, the molecule was strongly correlated with expression of ER $(\mathrm{p}<.0001)$ and $\mathrm{PR}(\mathrm{p}=.0008)$, and inversely associated with tumor grade $(\mathrm{p}<.0001)$. Reflecting these findings, GATA3 levels in luminal BCs were significantly higher than in the HER2 and TNBC subtypes $(\mathrm{p}<.0001)$, but were similar to that of the luminal controls. It is of further interest that $88 \%(23 / 26)$ ER-negative tumors showed GATA3 expression (H-score range, 10-275) and that the HER2 subtype had a significantly higher level of GATA3 expression than TNBCs $(\mathrm{p}=.009)$. Moreover, high GATA3 expression was a favorable prognostic indicator for overall survival as a continuous $(\mathrm{p}=.03)$ or dichotomized $(\mathrm{p}=.002)$ variable, and a significant prognosticator for relapse free survival as a dichotomized variable $(\mathrm{p}=.01)$

Conclusions: Although strongly associated with ER status, variable expression of GATA 3 was seen in nearly $90 \%$ of non-luminal BCs that are typically high grade. This argues against the previous findings that GATA3 acts solely as a luminal differentiation marker. The observation of significant higher levels of GATA3 expression in the HER2 subtype than in TNBCs may provide new insight into the pathogenesis of BC. Further, GATA3 expression provides prognostic significance in advanced BCs along with other conventional variables, and thus may be utilized in assisting clinical decision making. Cancer: Factors Associated with Residual Disease in Re-Excision

S McHugh, O Tawfik, F Fan. University of Kansas Medical Center, Kansas City, KS Background: Previous studies have attempted to identify factors such as the size and extent of invasive and in-situ carcinoma, and extent of positive/close margins that are associated with re-excision and the presence of residual disease in the re-excision specimen for breast cancer. Controversy still exists on the definition of positive/close margins and the need for re-excision. In this study, we retrospectively reviewed all lumpectomy cases for invasive breast carcinoma that were followed by re-excisions. The aim was to identify factors that were associated with presence of residual disease in re-excisions.

Design: A retrospective chart review was performed for the time period between July 2002 and July 2013 to identify patients who underwent lumpectomy for invasive mammary carcinoma followed by re-excision. Primary tumor characteristics including tumor size, grade, lymph node status, and margin status were collected and categorized. Margin status was divided into positive (tumor on ink), close $(<2 \mathrm{~mm})$ and negative $(>2 \mathrm{~mm})$. These parameters were compared between cases with residual carcinoma and cases without residual carcinoma in re-excisions

Results: Among 845 cases that had lumpectomy for invasive carcinomas, 117 (14\%) were identified as having subsequent re-excision. The results are shown in table 1. 


\begin{tabular}{|c|c|c|c|c|}
\hline & & $\begin{array}{l}\text { With residual } \\
\text { carcinoma }(n=37)\end{array}$ & $\begin{array}{l}\text { Without residual } \\
\text { carcinoma }(\mathrm{n}=80)\end{array}$ & p value* \\
\hline \multirow[t]{2}{*}{ Age (yrs) } & $\geq 50(\mathrm{n}=93)$ & 29 & 64 & 0.839 \\
\hline & $<50(\mathrm{n}=24)$ & 8 & 64 & \\
\hline \multirow[t]{2}{*}{ Invasive tumor size $(\mathrm{cm})$} & $\leq 2(\mathrm{n}=101)$ & 29 & 72 & 0.089 \\
\hline & $>2(n=16)$ & 8 & 8 & \\
\hline \multirow[t]{4}{*}{ Invasive tumor grade } & $\mathrm{I}(\mathrm{n}=26)$ & 8 & 18 & 0.727 \\
\hline & II $(\mathrm{n}=64)$ & 22 & 42 & \\
\hline & III $(\mathrm{n}=25)$ & 7 & 18 & \\
\hline & Unknown & 0 & 2 & \\
\hline \multirow{3}{*}{$\begin{array}{l}\text { Extensive in-situ } \\
\text { component }\end{array}$} & Present $(\mathrm{n}=33)$ & 14 & 19 & 0.119 \\
\hline & Absent $(\mathrm{n}=24)$ & 4 & 20 & \\
\hline & Unknown $(\mathrm{n}=60)$ & 19 & 41 & \\
\hline \multirow[t]{4}{*}{$\begin{array}{l}\text { Ductal carcinoma in- } \\
\text { situ grade }\end{array}$} & Low $(\mathrm{n}=10)$ & 3 & 7 & 0.713 \\
\hline & Intermediate $(\mathrm{n}=27)$ & 11 & 16 & \\
\hline & High $(n=34)$ & 10 & 24 & \\
\hline & Unknown $(n=46)$ & 13 & 33 & \\
\hline \multirow[t]{3}{*}{$\begin{array}{l}\text { Margins for invasive } \\
\text { and/or in-situ } \\
\text { carcinoma }\end{array}$} & Positive $(\mathrm{n}=46)$ & 21 & 25 & 0.032 \\
\hline & $\leq 2 \mathrm{~mm}(\mathrm{n}=62)$ & 14 & 48 & \\
\hline & $>2 \mathrm{~mm}(\mathrm{n}=9)$ & 2 & 7 & \\
\hline \multirow[t]{3}{*}{$\begin{array}{l}\begin{array}{l}\text { Number of positive/ } \\
\text { close margins }(n=108)\end{array} \\
\end{array}$} & $\mid 1$ & 8 & 34 & 0.001 \\
\hline & 2 & 8 & 26 & \\
\hline & $\geq 3$ & 19 & 13 & \\
\hline \multirow[t]{2}{*}{$\begin{array}{l}\text { Lymphovascular } \\
\text { invasion }\end{array}$} & Present $(n=17)$ & 7 & 10 & 0.359 \\
\hline & Absent $(\mathrm{n}=100)$ & 30 & 70 & \\
\hline \multirow[t]{4}{*}{ Axillary lymph node } & No $(n=76,2$ ITC $)$ & 22 & 54 & 0.539 \\
\hline & N1a $(n=21)$ & 7 & 14 & \\
\hline & $\mathrm{N} 1 \mathrm{~b}(\mathrm{n}=5)$ & 3 & 2 & \\
\hline & Unknown $(\mathrm{n}=15)$ & 5 & 10 & \\
\hline
\end{tabular}

* Chi-square, $\mathrm{p}<0.05$

Conclusions: The distance of invasive/in-situ carcinoma from margin (positive, $\leq 2 \mathrm{~mm}$ and $>2 \mathrm{~mm}$ ) and the number of positive and/or close margins are significantly $(\mathrm{p}<0.05)$ associated with the presence or absence of residual carcinoma in the re-excisions. 2 $\mathrm{mm}$ or less is an adequate cut-off measurement for close margin. Positive and/or close margins, especially when multiple, warrant re-excision.

262 Atypical Lobular Hyperplasia: A Review of Follow Up Surgical Treatment

S Mehrotra, JA Wisell, SB Sams. University of Colorado School of Medicine, Aurora, CO.

Background: Lobular neoplasia (LN) of the breast is a monomorphic proliferation, including atypical lobular hyperplasia (ALH) and classic lobular carcinoma in situ (LCIS). Histologically there is a proliferative gradation from ALH to LCIS, depending upon the extent of involvement of the acini. Epidemiological studies demonstrate that LCIS imparts a significant increased relative risk of subsequent invasive carcinoma and recent molecular studies support the concept of LCIS as a non-obligate precursor of breast cancer. LN is typically an incidental finding on core needle biopsies (CNB) targeting other lesions and given these silent features, the true prevalence and the rate of progression are unknown. Thus, clinical management of LN remains controversial, ranging from clinical/radiological follow up to obligatory surgical excision. At our institution ALH identified on CNB is managed with surgical excision.

Design: We performed a 5 year retrospective review of CNB with a diagnosis of ALH, subsequent surgical excision, and the mammography findings in correlation with the targeted lesion in terms of calcifications or mass lesions. Biopsies with additional diagnoses of atypical ductal hyperplasia, DCIS, invasive tumors or other entities warranting an excision were excluded. The slides were reviewed by a board certified pathologist and pathology fellow to confirm the diagnosis. Reports of subsequent excisions were reviewed for upgraded diagnoses including DCIS or invasive carcinoma. Results: 20 cases of ALH on CNB with subsequent excisions were identified. 18 cases were targeted for calcifications and 2 for asymmetrical mammography findings without calcifications. Microcalcifications were confirmed on biopsy in all 18 cases. Diagnoses on surgical excision were as follows: benign (38\%), ALH (42\%) and LCIS (14.2\%). None of the cases had invasive cancer or DCIS.

Conclusions: All cases on excision contained lesions not requiring additional surgical management. The rate of upgrade to carcinoma in our study was $0 \%$ and in the literature ranges from $2-40 \%$. Arguably, this variation arises from the incidental finding of LN associated with other lesions such as ductal hyperplasia. In our limited study, 18 of 20 cases had calcifications, confirmed present in the biopsy, indicative that the targeted lesion was sampled. We propose biopsies with diagnosis of ALH alone and a strong imaging correlation confirming the targeted lesion are best managed clinically in lieu of surgical excision. We acknowledge that confirmation of sampling of mass-like asymmetries can be more difficult to determine and may warrant an excisional biopsy.

Cytokeratin 17Trends toward Overexpression in Triple-Negative Breast Carcinoma but Is Unrelated to Sox2 Expression

RD Merkin, JL Romeiser, CR Rizk, S Burke, AL Shroyer, KR Shroyer. Stony Brook University School of Medicine, Stony Brook, NY.

Background: Triple-negative breast carcinomas (TNBC) (ER-, PR-, Her2-) is a particularly challenging subtype of breast carcinoma to treat, as they are unresponsive to targeted therapies that utilize the estrogen and Her2 receptors, resulting in poorer survival outcome for patients. In our lab, cytokeratin 17 (Krt17) has been recently identified by mass spectrometry and confirmed with immunohistochemistry (IHC) as a prognostic marker of squamous cell carcinomas of the cervix, and may be an important marker in other carcinomas as well. Sox 2 is a marker of pluripotency in cells, and is believed to play a role in breast tumorigenesis. This study focused on invasive cancers, and sought to examine the hypotheses that 1) Krt17 expression is increased in TNBC relative to non-TNBC, and 2) that Sox 2 expression is related to Krt17 expression.

Design: Forty four cases of invasive breast carcinoma ( $\mathrm{n}=38$ invasive ductal carcinoma [IDC], $\mathrm{n}=6$ invasive lobular carcinoma [ILC]), treated with surgical excision of tumor at our University Hospital, were identified through the New York Cancer Registry. Formalin-fixed, paraffin embedded tumor specimens were retrieved from the UH archives. Representative sections of each tumor were used to localize Krt17 and Sox2 using indirect immunoperoxidase methods. Cytoplasmic, $2+$ staining $\geq 5 \%$ of the tumor cells was scored positive for Krt17; any nuclear staining was scored positive for Sox 2 . Fisher's exact tests were used to detect differences between TNBC and non-TNBC specimens. McNemar's test was used to examine differences in Krt17 and Sox2 expression in all specimens.

Results: There was no significant difference in Krt17 staining between ILCs and IDCs $(\mathrm{p}=0.57)$. Staining was detected in $18.2 \%(8 / 44)$ of all specimens; $50 \%(3 / 6)$ of TNBCs showed positive expression compared to $13.2 \%(5 / 38)$ of non-TNBCs $(\mathrm{p}=0.06)$. Sox 2 was detected in $36 \%(16 / 44)$ of all specimens; $50 \%$ (3/6) of TNBCs showed positive expression compared to $34.2 \%(13 / 38)$ of non-TNBCs $(p=0.65)$. Sox 2 expression was not correlated with $\mathrm{Krt17}$ expression $(\mathrm{p}=0.12)$.

Conclusions: In summary, this study suggests that Krt17 might show a greater expression in TNBC as compared to non-TNBC, but a larger sample size is needed. Krt17 does not appear to be related to Sox 2 expression, thus, Krt17 expression appears to be unrelated to stem cell-like properties of breast carcinoma. Further studies are underway to determine if $\mathrm{Krt17}$ has prognostic significance for tumor recurrence and patient survival.

264 Biopathological Signification of the Long Intergenic Non Coding RNA TUG1 Dysregulation across Invasive Breast Carcinomas

D Meseure, K Drak Alsibai, S Vacher, E Martel, S Arrufat, A Nicolas, M Caly, $M$ Trassard, X Sastre, I Bieche. Curie Institute, paris, France.

Background: Long non coding RNAs (lncRNAs) orchestrate coordinated spatiotemporal regulatory patterns of gene expression in association with distinct architectural nuclear structures. Pc2 (Polycomb 2 protein) is a reader for the histone code and its methylation/demethylation controls relocation of genes between nuclear Polycomb bodies (PcBs) where their expression is repressed and interchromatin granules (ICGs, speckles) where their expression is activated.This movement is the consequence of binding of Pc2 to two lncRNAs, TUG1 (Taurine Up-regulated Gene 1) and MALATI (metastasis-associated lung adenocarcinoma transcript 1) respectively located in PcGs and ICGs.

Design: We quantified (i) TUG1 and MALATI at RNA level using RT-PCR and RNAFISH in a series of 456 invasive breast carcinomas (IBCs) and at protein level using immunohistochemistry in a series of $90 \mathrm{IBCs}$, (ii) TUG1 and MALAT1 regulators (RNPS1, SON, PRPG) and coactivators (LSD1, MLL, SETD2), (iii) components of speckles (SRSF1, SRSF2, SRSF3), and Polycomb PRC1 complex (MEL18, BMI1, $C B X 4 / 8, R I N G 1 A / B)$, (iv) genes involved in the cell cycle (E2F1, B-MYB, $p 14 / A R F$, p15, p16, p21), apoptosis (caspase3/8/9, BCL2, BAX, BCLXL, BIRC6), EMT (SNAI1, $S N A I 2, Z E B 1, Z E B 2)$, migration (AIM1, LAYN, HMMR) and metastatic spread (ROBO1, GPC6, LPHN2, CDCP1, ABCA1, DRD1, COL6A1)

Results: Overexpression of TUG1 and MALATI in IBCs were (i) associated with dysregulation of speckles components and $P R C l$, (ii) observed in all IBCs subtypes, particularly in $\mathrm{HR}+$ tumors and (iii) significantly correlated with overexpression of transcripts involved in cell cycle proliferation, survival but of most importance in EMT, invasion and metastatic spread.

Conclusions: Dysregulation of the two $\operatorname{lnc} R N A s$ TUG1 and MALATI in IBCs seems to disrupt the function of the nuclear bivalent platform represented by ICGs and PcBs, which coordinates an extensive multigene transcriptional program implicated in survival, EMT and metastatic spread of cancer cells.

\section{5 miRNA Expression Pattern in Predicting BRCA Mutation Status} in Invasive Breast Carcinoma

SK Mohanty, G Liles, S Thakur, SS Sahu, K Kalra, MB Amin, RK Pillai, F Dadmanesh. Cedars-Sinai Medical Center, Los Angeles, CA; BioGenex Laboratories, Fremont, CA. Background: MicroRNAs (miRNAs) are short, non-coding RNAs involved in posttranscriptional regulation of gene expression by affecting the stability and translation of mRNAs. It has been shown that miRNAs are aberrantly expressed in breast cancers (BC). Three miRNAs have been associated with breast tumorigenesis: miR21, let-7a and miR17. Although there is limited data for the utility of immunohistochemical (IHC) markers for predicting BRCA mutation status in BC, there is no data available for in situ expression of miRNA within FFPE tissue of BRCA-related BC. Thus, we sought to correlate in situ expression of miR21, let-7a and miR17 with BRCA mutation status in invasive BC

Design: We selected 13 cases of invasive ductal carcinoma: 6 BRCA and 7 sporadic. In situ hybridization using FAM-labeled miRNA probes (BioGenex) for let-7a, miR21 and miR17 was performed. The staining results were evaluated in the tumor and benign epithelial and myoepithelial nuclei as weak, moderate or strong; percent positivity was estimated as focal $(<50 \%)$ or diffuse $(>50 \%)$. 
Results: Tumor expression of miRNA is summarized in Figure 1.

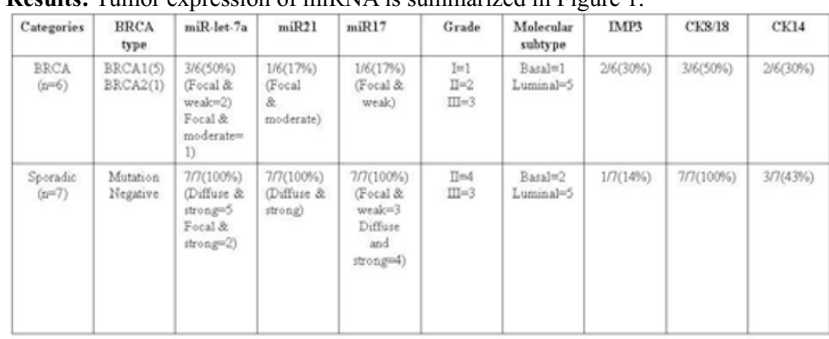

In the sporadic group, the expression of $\operatorname{miR} 21, \operatorname{miR} 17$, and let-7a in normal epithelium and myoepithelium is strong and uniform, whereas the expression is focal and weak in the BRCA group.

Conclusions: In contrast to microarray-based miRNA studies, we evaluate in situ expression of miRNA within FFPE BC tissue, where histologic features are wellmaintained. Compared to sporadic BC, expression of let-7a, miR21 and miR17 is downregulated in BRCA-related BC. Of note, both of the miR21 and let-7a + cases in the BRCA group are Luminal A, suggesting an estrogen receptor linkage. Underexpression of these miRNA markers may favor a BRCA-mutated phenotype in BC and support an alternative pathway of oncogenesis in BRCA-related BC. Ongoing studies in a larger cohort are underway to fully assess the potential of these miRNAs in predicting BRCA mutation status. The ability to interpret miRNA expression patterns may help more accurately determine patients who require BRCA genetic testing, which is both costly and time consuming.

266 Identification of Single Nucleotide Variations in the ESR1 Ligand Binding Domain for OH-Tamoxifen in ER(+), Treatment Naïve Breast Cancers

P Mojica, D Sykes, W Mojica. UCLA, Los Angeles, CA; University at Buffalo, Buffalo, NY.

Background: Estrogen receptor positive $(\mathrm{ER}+)$ breast cancer patients are candidates for targeted adjuvant therapy with selective estrogen receptor modulators like tamoxifen. While the majority of treated patients have a favorable response, a subset demonstrate recurrent disease. Several escape mechanisms have been postulated to account for this, however relatively little attention has been paid to identifying variants in the estrogen receptor gene's (ESR1) highly conserved ligand binding domain (LBD) as a possible reason for refractory disease. Variations in nucleotide sequence can affect the binding affinity of therapeutic agents by reducing the number of favorable van der Waals contacts, creating unfavorable electrostatic interactions or through an increase in steric hindrance.

Design: The dbSNP database was queried for regions responsible for binding the active form of tamoxifen. Four separate primer pairs were designed to encompass the critical amino acids responsible for $\mathrm{OH}$-tamoxifen binding from a previous crystallography study. Twelve cases of ER+ breast cancer were identified and tissue blocks with $>80 \%$ tumor content selected. DNA was extracted from formalin fixed, paraffin embedded tissue sections and subjected to the polymerase chain reaction creating 48 different samples. The resulting amplicons were purified and sequenced, followed by basic local alignment analysis to the most recent NCBI homo sapien gene annotation for the ESR1 gene (GI:201023303)

Results: No variants are documented in the dbSNP database for the amino acids involved in the $\mathrm{OH}$-tamoxfen binding to the ESR1 protein. Two samples in this study failed to generate interpretable sequences. Three cases exhibited a silent variant change in the same location g.413209A $>$ C. One case had a variant (g.413259 A $>$ T) that resulted in an amino acid substitution (asparagine for tyrosine) adjacent to an amino acid implicated by crystallography to be involved in the van der Waals binding of OH-Tamoxifen to the ESR1 protein binding pocket. One case had a variant resulting in the substitution of valine for glutamic acid (g.413281A $>$ T). All variations were found in exon 10 . Conclusions: In this small study a surprising number of variations were identified in the highly conserved ESR1 LBD. Those cases with variations will require comparison with matched control tissue to establish whether these variants represent polymorphisms or mutations. Clinical follow-up and functional studies are required to determine if these variants have clinical implications.

267 Transcriptome-Wide Association of MKI67 Expression: Pre- and Post-Neoadjuvant Paclitaxe

AM Moyer, KR Kalari, KJ Thompson, DW Visscher, VJ Suman, J Eckel-Passow, $H$ Sicotte, SN Hart, JP Sinnwell, TJ Dockter, KN Jones, AL Conners, X Tang, J Yu, B Gao, DW Mahoney, P Barman, P Vedell, SA McLaughlin, A Moreno Aspitia, DW Northfelt, RJ Gray, ED Wieben, G Farrugia, C Schultz, JN Ingle, L Wang, JC Boughey, RM Weinshilboum, MP Goetz. Mayo Clinic, Rochester, MN; Mayo Clinic, Jacksonville, FL; Mayo Clinic, Scottsdale, AZ

Background: KI67, a marker of proliferation, is used in prognosis of breast cancer and incorporated into clinical multi-gene expression panels. However, the underlying biology and function of the MKI67 protein is not well understood. Neoadjuvant chemotherapy (NAC) is the standard of care for locally advanced breast cancer and elevated KI67 post NAC is associated with poor prognosis. The impact of NAC on KI67 index and protein function has not yet been fully explored. We sought to identify mRNAs with expression correlated with expression of MKI67 and to determine if that relationship is altered by NAC to better understand the function and relationships of MKI67.

Design: RNA was extracted from core biopsies of triple negative and luminal B breast tumors from women enrolled in the Mayo Breast Cancer Genome-Guided Therapy
(BEAUTY) study before $(\mathrm{n}=18)$ and after $(\mathrm{n}=16) 12$ weeks of neoadjuvant paclitaxel. The median baseline KI67 (by IHC) was $45 \%$ and decreased to $14 \%$ post paclitaxel. RNAseq was performed using the Illumina HiSeq2000 and the Mayo Analysis Pipeline for RNAseq (MAP-Rseq) for quality control, sequence alignment, and gene counts. After normalization, MKI67 expression was evaluated for association with transcripts across the transcriptome. Ingenuity Pathway Analysis Software was used to identify pathways containing the transcripts of interest.

Results: MKI67 expression showed highest association with the "axonal guidance signaling" pathway, pre- and post-NAC ( $\mathrm{p}=6.28 \mathrm{E}-11$ and $5.51 \mathrm{E}-07$, respectively). Comparing the top 100 transcripts associated with MKI67 expression (positively, $r>0.796$ and negatively, $r<-0.764$ ), 55 transcripts overlapped between the two groups, whereas 145 transcripts were unique to each group. Analysis of the unique transcripts showed pre-treatment MKI67 expression was associated with increased expression of the "role of CHF proteins in cell cycle checkpoint control" $(\mathrm{p}=5.47 \mathrm{E}-05)$ and "role of BRCA1 in DNA damage response" ( $p=8.34 \mathrm{E}-05)$ pathways, while in the post-NAC group, MKI67 expression was most associated with increased expression of the "mitotic roles of polo-like kinase" pathway $(\mathrm{p}=2.78 \mathrm{E}-07)$.

Conclusions: In this exploratory study, we identified differently expressed genes and pathways associated with $M K I 67$ expression, pre- and post-paclitaxel. These results raise the possibility that the biological function of MKI67 may be altered by NAC. Further studies are required to explore this hypothesis and to identify new treatment strategies, given that elevated $\mathrm{KI} 67$ post-NAC is associated with poor prognosis.

\section{Tumor-Infiltrating T-Bet-Positive Th1 Lymphocytes Predict} Outcome in Lymph Node Negative Breast Cancer

AM Mulligan, D Pinnaduwage, S Tchatchou, S Bull, I Andrulis. University Health Network, Toronto, ON, Canada; Mount Sinai Hospital, Toronto, ON, Canada.

Background: Interactions of tumor cells with their microenvironment may influence tumor growth and progression. The presence of tumor specific $\mathrm{T}$ cells has been correlated with improved outcome in different cancers; however, certain T cells can promote the progression of tumors through different growth factors. We hypothesized that lymphocytes expressing T-box transcription factor (T-bet), a transcription factor essential for Th1 development and IFN- $\gamma$ production, would confer a favorable prognosis in breast cancer.

Design: The cohort comprised a prospectively accrued consecutive series of women with lymph node negative breast carcinoma from eight hospitals. Tissue blocks were available for tissue microarray construction in 887 women. Patients were followed for recurrence and death. Estrogen and progesterone receptor status was determined biochemically at the time of surgery. HER2, Ki67, CK5, EGFR, p53 and T-bet status was determined using immunohistochemistry. Tumors were assigned to luminal A, luminal B, HER2 and basal subgroups based on previous studies. The number of T-bet+ lymphocytes were counted in tumor nests (intratumoral), in stroma immediately adjacent to tumor cells, and in stroma distant to tumor cells. Tumors were dichotomized into T-bet-high and T-bet-low based on the number of intratumoral and immediately adjacent stromal T-bet + lymphocytes using a cut-off chosen without knowledge of clinical outcome. Results: Patients with T-bet-high tumors had larger $(\mathrm{p}=0.0158)$ and higher grade tumors $(p<0.0001)$ and were more likely to have received chemotherapy $(p<0.0001)$. These tumors were also significantly associated with hormone receptor negativity, CK5, EGFR and p53 positivity, high Ki67 labelling index and basal subgroup. Using the Cox proportional hazards model, in univariate analysis (median follow-up of 96.5 months) T-bet-low tumor status was associated with a reduced disease free survival compared with T-bet-high tumor status ( $\mathrm{p}=0.0526, \mathrm{RR}=7.03,95 \% \mathrm{CI}: 0.98-50.45)$. This finding was retained in multivariate analysis that included traditional clinicopathologic factors and HER2 ( $\mathrm{p}=0.0373, \mathrm{RR}=8.33,95 \% \mathrm{CI}: 1.13-61.27$ ).

Conclusions: Despite being associated with adverse clinical-pathologic variables, high numbers of T-bet + lymphocytes in breast cancers are associated with a favorable outcome compared with low numbers of T-bet+ lymphocytes. This supports their role in Th1-mediated anti-tumor activity and may provide insight for the development of new therapeutic strategies.

269 Androgen Receptor Expression in Subtypes of Breast Ductal Cancer in African American Women

TJ Naab, LJ Ricks-Santi, Y Kanaan, AK Esnakula. Howard University Hospital, Washington, DC; Hampton University, Hampton, VA; Washington University, St Louis, MO.

Background: The triple negative (TN) breast cancers lack specific molecular targeted therapies. It has been shown that approximately $10-35 \%$ of tumors without ER/PR expression or HER2 amplification (TN breast carcinomas) are associated with androgen receptor (AR) expression. Thus, AR may represent a therapeutic target in $\mathrm{TN}$ breast cancers, which have an increased incidence in African American women. The objective of our study is to correlate the immunohistochemical expression of AR in the four major subtypes of breast carcinoma (Luminal A, Luminal B, HER2, and Triple Negative) in a cohort of African American women with other clinicopathological factors.

Design: Tissue microarrays (TMAs) were constructed tumor blocks from primary ductal breast carcinomas in 203 African-American females. Two separate $1 \mathrm{~mm}$ cores represented each case. The polymer-HRP system was utilized for immunostaining. Five micrometer sections are stained with a mouse monoclonal antibody against the androgen receptor (AR441, Dako, CA). The sections are evaluated for the intensity of nuclear reactivity (0-3) and the percentage of reactive cells; an H-score is derived from the product of these measurements. Cases are categorized as having negative/ weak (score $<10$ ) or positive (score $>10$ ) expression. Bivariate analysis is done via $\chi^{2}$ analysis and survivability data is calculated via the generation of Kaplan-Meier curves (SPSS v19). Statistical significance was assumed if $\mathrm{P}<0.05$. 
Results: AR expression was significantly associated with lower grade $(\mathrm{p}<0.001)$, ER positivity ( $\mathrm{p}<0.001)$, PR positivity ( $\mathrm{p}<0.001)$, and Luminal A and $\mathrm{B}$ tumors $(\mathrm{p}<0.001)$. AR expression was found in $24 \%$ (15/62) of TN breast cancers. No significant correlation was seen with survival.

Conclusions: In our study, AR expression is associated with lower grade, ER positivity, and PR positivity. However, it is expressed in $24 \%$ of TN cases. Few studies have looked at the utility of AR as a prognostic or predictive marker in breast cancer. AR expression has been associated with a clinical response of advanced breast cancers to medroxyprogesterone acetate and has been shown to be more highly expressed in cancers with a specific PIK3CA mutation. AR may represent a target for therapy, probably in combination with other agents

\section{Concurrent Molecular Targeting of Raf and Mek in Triple} Negative Breast Cancer

TS Nagaria, C Leduc, S Sikdar, PA Greer, W Sangrar. Division of Cancer Biology and Genetics, Queen's Cancer Research Institute, Queen's University, Kingston, ON, Canada.

Background: Despite advances in predictive testing in breast cancer, there has been minimal improvement in stratifying patients with triple negative breast cancer (TNBC). A substantial proportion of TNBCs harbor aberrations in the mitogen activated protein kinase (MAPK) pathway. With the advent of small molecule inhibitors, there is a clear potential for these agents in the treatment of TNBC as well as a possible role for predictive molecular testing at the time of tissue diagnosis. Here, we explore combination treatment of TNBC with small molecule inhibitors of Raf and Mek using both in vitro and in vivo models.

Design: TNBC cell line MDA-MD-231 (MDA) with mutations in $K R A S$ and $B R A F$ was used for MTT cytotoxicity assay with treatments as follows: Raf inhibitor Sorafenib (SFN); Mek inhibitor U0126; SFN with fixed doses of U0126; control. Drug interactions were analysed using COMPUSYN software to generate drug effect-combination indices (CIs): $<0.9$, synergy; 0.9-1.1, additivity; $>1.1$, antagonism. MDA cells were xenografted into mammary fat pads of BalbC-RAG2 ${ }^{-/}\left[\mathrm{IL} 2 \mathrm{R} \gamma \mathrm{c}^{-/}\right.$mice $(n=24)$ that were randomized for treatment: SFN; Mek inhibitor AZD6244 (AZD); SFN+AZD; or PBS (control). Primary tumor and lungs were harvested at day 37 for histologic analysis, and immunoblotting was performed to assess MAPK signalling.

Results: Sorafenib exhibited strong cytotoxicity in vitro $\left(K_{\mathrm{Eff}} 99 \%, \mathrm{EC}_{50} 10.3 \mu \mathrm{M} \pm 0.83\right.$ SEM). U0126 potentiated SFN cytotoxicity at low doses with CIs in the synergistic range $(<0.9)$. In vivo, SFN+AZD showed greatest suppression of both primary tumour growth and metastases compared to single agents: Primary tumour, SFN+AZD vs control, SFN or AZD $(p<0.001,2$-way ANOVA, shown in Figure 1$)$; metastases, SFN+AZD vs control $(p=0.0002, t$-test $)$. SFN+AZD strongly suppressed Mek, Erk, Rb activity in tumour lysates suggesting decreased MAPK signalling in vivo.

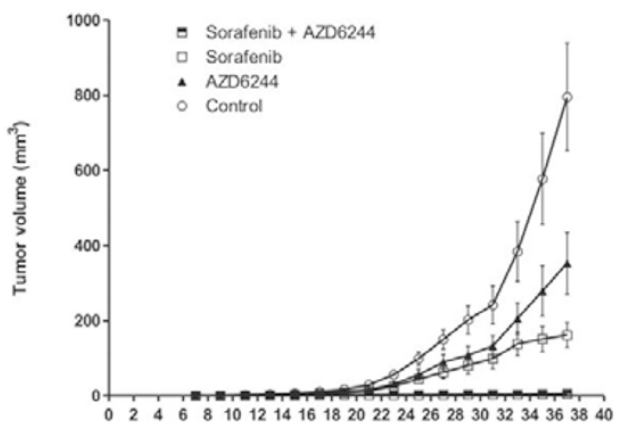

Time (days)

Figurel: Combined treatment with Sorafenib and AZD6244 results in enhanced tumor suppression in viro. Shown are estimates of mean tumor volume \pm SEM: SEN + AZD vi SEN or AZD or control $(P<0.0001 ; 2$-way

Conclusions: Stratifying patients with TNBC remains challenging, in part due to limited treatment options. This study demonstrates the therapeutic potential of concurrent Raf and Mek inhibition in a MAPK-dependent TNBC model, which suggests a role for molecular profiling at diagnosis.

\section{1}

Optimizing MIB-1 Detection in Breast Cancer: Is Digital Imaging Accurate?

KK Narra, ME Salama, RE Factor. University of Utah, Salt Lake City, UT.

Background: Ki67 is a marker of proliferation detectable by immunohistochemistry using the MIB-1 antibody. Recent clinical trials have shown that Ki67 has important prognostic and predictive significance in breast cancers, and exact percentages provide critical cut-offs for treatment decisions. At this time, the measurement of Ki67 has not been standardized. Our study objectives were to determine the accuracy of digital imaging for Ki67 measurements and to investigate whether a keratin/MIB-1 dual stain would improve the accuracy of the digital count, since cells other than malignant cells can stain positive for MIB-1

Design: Unstained slides from formalin-fixed breast tissue were obtained from an IRB-approved clinical trial that required a Ki67 score as a study endpoint. There were fourteen total cases, which underwent Ki67 assessment by four different methods: 1) "eyeballing" the result; 2) staining with MIB-1 and counting 1000 malignant cells using light microscopy in a designated area; $3+4$ ) digitally scanning H\&E and MIB-1 stained slides or MIB-1 and keratin AE1/AE3 (two color dual stain) with the Aperio XT Scanscope and quantitatively analyzing the same designated area with image analysis algorithms. For 2-4, a positive result was reported as a percent of positively stained malignant cells out of the total examined. Statistical analysis to compare the different measuring methods was performed using the Pearson Correlation Coefficient.

Results: Manual count of Ki67 stained tumor was considered the gold standard. Eyeballing demonstrated the closest correlation $(r=0.9019, p<0.0001)$, digital imaging of the Ki67 stain demonstrated close correlation $(\mathrm{r}=0.8296, \mathrm{p}<0.0002)$, and manual counting with the keratin and MIB-1 dual stain also showed statistically significant correlation $(r=0.7151, p<0.004)$. Digital imaging of the dual stained slides showed poor correlation to the gold standard $(r=-0.04378, p=0.8819)$. The computer recognition of the dual stain was complicated both by technical issues, such as crush artifact and cytoplasmic staining, and by tumor characteristics whereby some cells that were clearly malignant did not stain, suggesting that the same tumor may have heterogeneous staining due to differences in differentiation.

Conclusions: Eyeballing and digital imaging of MIB-1 strongly correlate with manual counts of the MIB-1 stained breast tumor. A dual stain with keratin and MIB-1 demonstrates poor correlation and is not a useful method to distinguish Ki67 in tumor versus non-tumor cells.

272 Radial Scar/Complex Sclerosing Lesions: A Clinicopathologic Correlation Study from a Single Institution

A Nassar, AL Conners, HJ Tina. Mayo Clinic, Jacksonville, FL; Mayo Clinic, Rochester, $\mathrm{MN}$.

Background: Radial scars (RS) or complex sclerosing lesions (CSL) of the breast are benign radiological and histological entities. Radiologically, they appear as architectural distortion with central radiolucency. These stellate lesions are frequently identified on screening mammography and, with the introduction of population-based screening programmes, their incidence has increased to $0.03 \%-0.09 \%$ of all core needle biopsies (CNB). They can, however, pose diagnostic difficulty as their radiological and histological appearance mimic carcinoma. Because of the high incidence of atypia or associated occult malignancy, the current literature recommendation is excision of all mammographically detected RS/CSL diagnosed on CNB.

Design: Our aim was to review all RS/CSL that were previously diagnosed on imageguided CNB from $1 / 94$ to $8 / 13$, and assess the pathology from the excisional biopsy to identify cases upstaged to atypia or neoplasm.

Results: There were 135 cases of radial scar without concomitant atypia on CNB diagnosed during that period. Average age of women is 54 years (range: $23-87$ years). Laterality includes 55 cases on the right breast, and 80 cases on the left. Thirty-five (26\%) cases have follow-up excision; whereas $100(74 \%)$ cases did not have. The average size of the excised radial scars is $2.4 \mathrm{~cm}(1.1-3.6 \mathrm{~cm})$. Almost two-third of excised cases $(66.7 \% ; 20 / 33)$ were greater than $1.0 \mathrm{~cm}$. The mammographic and ultrasonographic imaging features were listed as architectural distortion in $64.7 \%(22 / 34)$ and hypoechoic nodules with irregular margins in $84.8 \%(28 / 33)$ respectively. Almost all excised cases $(97.1 \% ; 34 / 35)$ showed radiologic and pathologic discordance, and are designated as Bi-Rads level 4 (suspicious for malignancy). The follow-up excisional biopsies revealed: 7 (20\%) invasive mammary carcinomas (2 metaplastic carcinoma; 3 invasive ductal and 1 invasive lobular carcinomas); $2(6 \%)$ in-situ ductal carcinoma; 3 (9\%) atypical lobular hyperplasia; 19 (54\%) residual radial scars; and $4(11 \%)$ with no residual lesions on follow-up.

Conclusions: Follow-up excisional biopsy is warranted for RS/CSL specifically if they are larger than $1.0 \mathrm{~cm}$ with worrisome radiographic images or showed radiologic and pathologic discordance, as approximately one-quarter $(26 \%)$ of these cases will have an upgrade to in-situ or invasive carcinomas on follow-up.

\section{Atypical Duct Hyperplasia Close to Surgical Margins: Time to} Revisit the Surgical Practices

A Nayak, MG Hanna, SChaudhary, IJ Bleiweiss, CS Nagi, S Jaffer. Mount Sinai Medical Center and Icahn School of Medicine, New York, NY.

Background: Surgical management of ADH present close to or involving the breast excision margins is debated. We have noticed marked variation in the practice amongst surgeons. The purpose of this study was to evaluate the incidence of residual disease in re-excised cases and to investigate if there is high incidence of cancer recurrence in cases not undergoing surgical re-excision.

Design: A retrospective review of pathology database between 2001 and 2013 was performed to identify cases with ADH present close to (within 1-mm of) or involving the surgical excision margins. Clinical, pathologic and follow-up data was recorded. Slides were reviewed to confirm the diagnosis.

Results: We identified 117 lumpectomy cases (pure ADH, 56; DCIS, 33; invasive carcinoma, 28) where $\mathrm{ADH}$ was the highest risk lesion in relation to resection margins (at or within 1-mm). Of these, 48 cases underwent re-excision (pure ADH, 24; DCIS, 17 ; invasive carcinoma, 7). Residual ADH or carcinoma was found in $26(54 \%)$ cases on re-excision. Of the 24 pure ADH cases, 7 showed residual ADH, and 4 were upgraded to carcinoma (DCIS, 2; invasive carcinoma, 2) on re-excision. Of 17 DCIS cases, 6 had residual ADH and 6 DCIS. Of 7 invasive carcinoma cases, 2 had residual ADH and 1 residual DCIS. In total, residual ADH, DCIS or invasive carcinoma was identified in $15(31 \%), 9(19 \%)$ and $2(4 \%)$ cases respectively. Of the remaining 69 patients with no re-excision, follow-up was available in $39(57 \%)$ with a mean follow-up of 29 months (range, 4 to 91 months). Two (5\%) of these developed invasive carcinoma and $1(3 \%), \mathrm{ADH}$. In the re-excised group, follow-up was available in $34(71 \%)$ with a mean follow-up of 40 months (range, 2 to 136 months). One (3\%) of these developed invasive carcinoma, 1 (3\%) DCIS and $2(6 \%) \mathrm{ADH}$.

Conclusions: Our results show a high rate $(54 \%)$ of residual ADH or carcinoma on re-excision. The lack of difference in recurrence rate between the re-excised and non 
re-excised group may be due to short follow-up time in our study. We recommend that distance of margin from ADH should be reported in pathology reports, and cases with close margins (within 1-mm) should be considered for re-excision.

274 Metaplastic Breast Carcinomas Display Genomic and Transcriptomic Heterogeneity

CK Ng, B Weigelt, T Popova, O Mariani, A Vincent-Salomon, JS Reis-Filho. Memorial Sloan-Kettering Cancer Center, New York, NY; Institut Curie, Paris, France.

Background: Metaplastic breast carcinoma (MBC) is a rare histological type of breast cancer, preferentially of triple-negative phenotype. In addition to the 'intrinsic gene' subtypes (i.e. luminal A, luminal B, basal-like, HER2-enriched, normal breast-like and claudin-low), six transcriptomic subtypes of triple-negative breast cancer with therapeutic implications (i.e. basal-like 1, basal-like 2, immunomodulatory, luminal androgen receptor, mesenchymal-like and mesenchymal stem-like) have recently been described. Furthermore, SNP array-based methods have been employed to identify BRCA1-associated genomic instability signatures. Here we sought to define the molecular subtypes of MBCs using current microarray-based classifiers and to determine whether MBCs display gene copy number profiles consistent with those of $B R C A 1$-associated breast cancers.

Design: Thirty consecutive triple-negative MBCs were retrieved from the authors' institutions, and classified into three groups: MBCs with spindle cell metaplasia, with squamous metaplasia, and with heterologous elements. DNA and RNA extracted from frozen sections with tumor cell content $>50 \%$ were subjected to gene expression (Illumina HumanHT-12 v4) and copy number profiling (Affymetrix, SNP 6.0), respectively. 'Intrinsic subtyping' was performed using a microarray-based version of PAM50, and the triple-negative subtyping using a dedicated website (http://cbc. mc.vanderbilt.edu/tnbc/). An algorithm to identify and quantify large-scale state transitions was applied to SNP6 data to stratify MBCs into BRCA1-like or nonBRCA1-like subtypes

Results: All but one MBCs with spindle cell metaplasia $(n=12)$ were of claudin-low subtype, whereas MBCs with squamous metaplasia $(n=10)$ or with heterologous elements $(n=8)$ were preferentially of basal-like subtype. The triple-negative subtyping revealed that no sample was classified as of luminal androgen receptor subtype. MBCs with spindle cell metaplasia were classified as mesenchymal stem-like $(n=5)$ or mesenchymal $(n=1)$ subtype, or were unclassified $(n=6)$. MBCs with heterologous elements were of mesenchymal $(n=8)$ subtype, whereas MBCs with squamous metaplasia were of mesenchymal $(n=3)$, basal-like $1(n=1)$ or basal-like $2(n=2)$ subtype, or were unclassified $(n=4)$. Nine of the 26 MBCs subjected to SNP6 analysis were classified as BRCA1-like.

Conclusions: The diversity of histological features of MBCs is reflected at the transcriptomic level, and only MBCs with spindle cell metaplasia were shown to be preferentially of claudin-low subtype. Only a subset (35\%) of MBCs display BRCA1like genomic profiles.

275 Subclassifying Lobular Carcinoma In-Situ (LCIS) Based on Phosphohistone H3 (PHH3)-Detected Mitotic Index

MT Nguyen, Z Chen, SJ Shin. Weill Cornell Medical College, New York, NY

Background: Pathologists report pleomorphic LCIS (PLCIS) as studies have suggested that this subtype may be biologically more aggressive than classical LCIS (CLCIS)

However, there is a significant grey area of interpretation since clear morphologic criteria have not been set. Also, other characteristics such as apocrine or signet ring features or florid growth pattern not uncommonly co-exist in PLCIS and whether these findings in the absence of high nuclear grade are sufficient to make the diagnosis is unclear. Subclassifying LCIS by another parameter such as mitotic index may be more accurate and reproducible, the biologic basis of which can be substantiated in part by results of recent findings that mitotic score was more prognostic than histologic grade in invasive lobular carcinomas (Rakha et al., Mod Pathol 2013). We set out to evaluate whether mitotic index as determined by $\mathrm{PHH} 3$, a mitotic specific immunostain, could differentiate variants of LCIS from CLCIS.

Design: Biopsies containing LCIS of 80 patients were identified in our files. Foci of PLCIS, LCIS with apocrine or signet ring features (apoLCIS), intermediate-high LCIS [those with florid pattern and/or intermediate-high (I-H) nuclear grade], and CLCIS were identified in each case. Corresponding unstained slides were stained with $\mathrm{PHH} 3$ (rabbit polyclonal, Abcam). The mitotic index (\# of mitoses per 1000 LCIS cells) was manually calculated in LCIS foci. Statistical analysis was performed.

Results: The mean, median, and standard deviation values for all LCIS subgroups are shown.

Table 1: Mitotic index* using PHH3 stain in subgroups of LCIS

\begin{tabular}{|l||l||l|l|l||}
\hline Histologic Subgroup & $\mathbf{N}$ & Mean & SD & Median \\
\hline CLCIS & 18 & 0.1 & 0.2 & 0 \\
\hline LCIS with I-H nuclear grade and/or florid growth pattern & 25 & 1.0 & 0.7 & 0.9 \\
\hline apoLCIS & 27 & 1.6 & 1.1 & 1.2 \\
\hline PLCIS & 10 & 4.2 & 4.1 & 2.7 \\
\hline
\end{tabular}

PLCIS

*Number of mitoses per 1000 lesional cells.

When comparing the mean mitotic indexes between each subgroup, there were statistically significant differences between CLCIS and PLCIS, CLCIS and apoLCIS, and CLCIS and I-H LCIS $(\mathrm{p}<0.05)$. There was no significant difference in the mitotic indexes amongst PLCIS, apoLCIS, and I-H LCIS subgroups.

Conclusions: PHH3-detected mitotic indexes can accurately subclassify LCIS and may be more reproducible than assessing by nuclear grade alone. LCIS with other traits such as apocrine features or florid growth pattern may be clinically comparable to PLCIS and when found, a diagnosis of PLCIS would be appropriate even in the absence of high nuclear grade.
276 Limitations of Breast Biomarkers Testing in Fine Needle Aspiration Cytology Preparation

S Nofech-Mozes, N Hosseini, S Noy, F-I Lu, E Slodkowska, G Han, W Hanna, Z Ghorab. Sunnybrook Health Sciences Centre, Toronto, ON, Canada.

Background: Receptor testing in primary and recurrent breast cancer is used to guide treatment decisions. Accurate and standardized testing methods are critical to ensure the proper classification of the patient's receptor status and evaluate the likelihood of response to endocrine and targeted therapy. According to the current practice recommendations testing has to be performed in formalin fixed material and any assay modification from the current protocol requires optimization and validation. In our institution Fine Needle Aspiration (FNA) cytology material is fixed in alcohol based preservative.

Design: Fifty one cytology specimens with malignant cells in cell blocks (CB) were retrieved (34 breast and 17 axillary FNAs). FNA samples were fixed in alcohol based preservative, followed by fixation in $10 \%$ neutral-buffered formalin. Immunocytochemistry for ER, PgR and HER2 (clones SP1, 1E2 and 4B5, respectively; Ventana Medical Systems, Tucson AZ, USA) was performed on CB. The status of ER, PgR and HER2 on CB was compared with the paired surgical pathology specimen. This set of cases was specifically enriched in HER-2 positive cases.

Results:

\begin{tabular}{|c|c|c|c|c|c|c|}
\hline & \multicolumn{2}{|c|}{ ER $(\mathrm{n}=48)$} & \multicolumn{2}{|c|}{$\operatorname{PgR}(\mathrm{n}=48)$} & \multicolumn{2}{|c|}{ HER $2 *(\mathrm{n}=31)$} \\
\hline & SS+ & SS- & SS+ & SS- & SS+ & SS- \\
\hline $\mathrm{CB}+$ & $23^{1}$ & 0 & $14^{2}$ & 0 & 12 & 0 \\
\hline CB- & $77^{3}$ & 18 & $\sqrt{7^{3}}$ & 27 & $\sqrt{3^{3}}$ & 16 \\
\hline
\end{tabular}

$\mathrm{CB}$ - cell block; $\mathrm{SS}$ - surgical specimen $;{ }^{1}$ - 3 cases were low positive, all were negative on cytology; ${ }^{2}-3$ cases were low positive, two were negative on cytology; ${ }^{3}$ - false negatives: * - cases with equivocal results on IHC were not included in the analysis since these are further tested by in situ hybridization. The specificity was $100 \%$ for all three markers. The sensitivity for ER, PgR and HER2 was $76.7 \%, 66.7 \%, 80 \%$, respectively. The negative predictive value was $72 \%$ (CI: $50.61-87.88$ ), $79.4 \%$ (CI: 62-91.26) and 84\% (CI: 60.4-96.43), respectively.

Conclusions: Negative results of breast biomarkers tested by immunocytochemistry on alcohol-fixed FNA preparations need to be interpreted with caution. However, positive results are concordant with surgical specimens in $100 \%$ of the cases. Other limitations include difficulty in distinction between in situ and invasive carcinoma, evaluation of paucicellular samples and availability of internal or appropriate external positive control.

277 High Agreement between Whole Slide Imaging and Optical Microscopy for Assessment of HER2 Expression in Breast Cancer

CB Nunes, RM Rocha, MA Buzelin, D Balabram, FS Foureaux, SS Porto, H Gobbi. Federal University of Minas Gerais, Belo Horizonte, Minas Gerais, Brazil; A.C. Camargo Hospital Cancer Center, São Paulo, Brazil.

Background: Whole slide imaging (WSI) technology has been used for training, teaching, researching, and remote consultation. Few studies compared HER2 expression using conventional optical microscopy (OM) and WSI evaluations in breast cancer. However, no consensus has been achieved comparing both assessments.

Design: Sections from tissue microarray containing 200 pre-selected invasive breast cancers were submitted to immunohistochemistry applying three anti-HER2 antibodies (DAKO HercepTest ${ }^{\mathrm{TM}}$, Novocastra CB11, Lab Vision SP3) and Dual colour dual hapten brightfield in situ hybridisation (DDISH, VENTANA INFORM HER2 Dual ISH assay). The immunostained TMA slides were scanned with a 20X microscope objective (Pannoramic MIDI, 3DHISTECH) and evaluated using OM and WSI (Pannoramic Viewer 1.15.2, 3DHISTECH), following the ASCO/CAP HER2 scoring guideline. HER2 expression agreement using both evaluations and sensitivity and specificity were calculated comparing the anti-HER2 antibodies and DDISH.

Results: WSI and OM HER2 evaluations agreement was considered good (SP3, $\mathrm{k}=0.78$; CB11, $\mathrm{k}=0.79$ ) to very good (HercepTest ${ }^{\mathrm{TM}}, \mathrm{k}=0.83$ ). WSI evaluation led to higher sensitivity (ranged from 98.5 of SP3 and HercepTest ${ }^{\mathrm{TM}}$ to 95.4 of CB11), and lower specificity (ranged from 86.2 of SP3 to 89.6 of HercepTest $^{\mathrm{TM}}$ ) compared to OM evaluation (sensitivity ranged from 89.0 of CB11 to 97.0 of SP3 and specificity ranged from 95.2 of SP3 - HercepTest ${ }^{\mathrm{TM}}$ to 97.1 of CB11).

Conclusions: High agreement was achieved between WSI and OM evaluations. All three antibodies were highly sensitive and specific using both types of evaluations. WSI can be considered an alternative tool for HER2 immunohistochemical assessment.

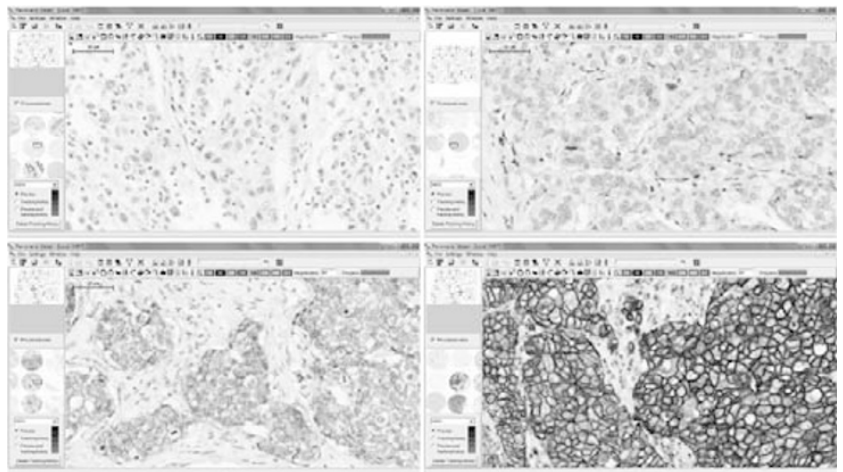

Figure 1 - Whole slide images of TMA cores displaying $0,1+, 2+$ and $3+$ HER2 scores following ASCO/CAP recommendations (SP3 antibody, 60X). 


\section{Radiographic Imaging Modalities in Invasive Mammary Carcinoma}

FD Oakley, HD Edwards, H Omar, Vanderbilt Breast Radiology. Vanderbilt University Medical Center, Nashville, TN.

Background: Tumor size is one of the most important factors in determining diseasefree and cause-specific survival in invasive mammary carcinoma. Increasing use of neoadjuvant and breast conserving therapies has also made it essential to have an accurate determination of this size prior to any definitive surgical intervention. Previous studies examining the correlation between imaging and breast pathology have been limited by size, number of imaging modalities examined and inclusion of final pathologic tumor stage (pT)

Design: A retrospective review of pathologic and radiologic data at our institution was performed to determine and explore the relationships between the pathologic tumor size/stage and radiologically determined tumor sizes of a consecutive series of 188 invasive mammary carcinomas.

Results: The best correlation with pathologic size was observed with MRI and the least with mammography (mammography 0.48 [151 cases], ultrasound (U/S) 0.66 [158 cases], MRI 0.70 [108 cases]). MRI was also most accurate in predicting the final pT score (MRI 61.1\%, mammography 57.6\%, U/S 55.1\% ). All modalities did worse predicting pT staging of small tumors, particularly in the case of stage pT1a $(\leq 0.5 \mathrm{~cm})$ where collectively only 2 out of 24 were correctly predicted. Of tumors $\leq 1 \mathrm{~cm}, U / \mathrm{S}$ most frequently gave the correct pT stage (U/S $47.3 \%$, mammography $40.0 \%$, and MRI $37.5 \%$ ), while for tumors $>1 \mathrm{~cm}$ MRI most frequently predicted the correct pT stage (MRI $71.1 \%$, mammography $68.8 \%, \mathrm{U} / \mathrm{S} 60.2 \%$ ). Of the 188 cases 66 had all 3 imaging modalities. MRI demonstrated the greatest linear correlation in the subset of cases where all 3 modalities were available (MRI 0.78, mammography $0.63, \mathrm{U} / \mathrm{S} 0.48$ ), whereas U/S had the lowest linear correlation. In the subset of cases with mammography, U/S and no MRI, U/S had a higher correlation (0.87), while the performance of mammography was similar (0.66). In cases where multiple imaging modalities predicted the same $\mathrm{pT}$ stage, the predictive value is higher. An agreement by all three modalities correctly predicted the final pT $74.4 \%$ of the time (vs mammography $68.2 \%$, U/S $57.6 \%$, MRI $66.7 \%$ ). Conclusions: A) Overall, mammography, U/S and MRI can correctly predict the true $\mathrm{pT}$ stage in only around $50 \%$ of the time an even less in tumors $\leq 0.5$. B) $U / S$ performed the best for tumors $\leq 1 \mathrm{~cm}$ and MRI performing best for tumors $>1 \mathrm{~cm}$. C) The predictive value of radiographic size in predicting the pT stage increases when more than one imaging modality predict the same stage.

\section{Extensive Rewiring of Epithelial-Stromal Crosstalk Networks} in Breast Cancer

E-Y Oh, SM Christensen, NW Knoblauch, L Montaser-Kouhsari, AH Beck. Beth Israel Deaconess Medical Center, Boston, MA.

Background: Epithelial-stromal crosstalk plays a critical role in cancer pathogenesis; however, little is known on a systems level about how epithelial-stromal interactions evolve during carcinogenesis. To address this issue, we developed an analytical framework for building genomewide epithelial-stromal crosstalk networks in the setting of breast carcinogenesis.

Design: We used expression profiling data derived from 72 laser captured epithelialstromal pairs from invasive breast cancer (IBC) and 41 pairs from normal breast available from five datasets in the Gene Expression Omnibus. We used the MatrixEQTL package in $\mathrm{R}$ to compute all pairwise associations of epithelial and stromal gene expression levels on 11,717 genes on ER+ IBC, ER- IBC, and normal breast. We selected the top $10 \mathrm{~K}$ strongest interactions for network analyses using Cytoscape, Network Analyzer, ClusterOneCytoscape, iGraph, and GOstats. Finally, for each analyzed gene, we computed survival analysis in ER+ and ER-negative IBC for a total of 4679 patients with publicly available clinically annotated expression profiling data.

Results: We identify 211 statistically significant epi-stromal interactions in normal breast, 1749 in ER+ IBC, and 2415 in ER-negative IBC at FDR's < 5\%. Among statistically significant interactions, we identified no self-loop interactions in normal breast, and a significantly higher proportion of self-loop interactions in ER-negative IBC $(210 / 2415(8.7 \%))$ and ER+ IBC $(332 / 1749(19.0 \%))($ All $\mathrm{P}<2.2 \mathrm{e}-16)$. The most statistically significant epithelial-stromal crosstalk interactions in IBC tend to be selfloop interactions, with $89 \%(\mathrm{ER}+)$ and $75 \%$ (ER-) of interactions significant at FDR $<0.01 \%$. Using the ClusterONE network analysis, we identify 219 highly connected clusters of genes in normal breast, 180 in ER-negative IBC, and 143 in ER+ IBC. Among the strongest epi-stromal crosstalk networks, we identify the highly connected KIF20AUBE2C-FOXM1-CCNB2-KIF4A sub-network in ER+ breast cancer, which we show to be strongly predictive of breast cancer patient survival $(\mathrm{HR}=1.43, \mathrm{P}=1.38 \mathrm{E}-07)$. Conclusions: Our analysis shows that epithelial-stromal crosstalk networks undergo extensive rewiring in invasive breast cancer leading to the emergence of epithelialstromal crosstalk network self-loops, hubs, and structured sub-networks enriched for biological hallmarks of carcinogenesis. This approach may facilitate the development of new diagnostics and therapeutics targeting epithelial-stromal crosstalk networks in cancer. Lesions (BSL)

E-Y Oh, LC Collins. Beth Israel Deaconess Medical Center, Boston, MA.

Background: Complex sclerosing lesions (CSL), radial scars (RS) and sclerosing papillary lesions (ScPap) of the breast are benign fibrosclerotic lesions that exhibit a wide spectrum of histologic appearances. One feature that characterizes BSLs is the presence of spindle cells within the sclerotic stroma. Metaplastic carcinomas, particularly those of the low grade fibromatosis-like type, may rarely arise in the setting of BSLs. However, it may be difficult to distinguish these sometimes subtle metaplastic carcinomas from the background BSL since both processes feature relatively bland spindle cells in a sclerotic stroma. Although immunostains for cytokeratins and/or p63 are observed in the spindle cells of metaplastic carcinomas, it is unclear whether these markers are also expressed in the stromal spindle cells of BSLs.

Design: To address this, we studied 55 CSL/RS and ScPap diagnosed between 20052012. H\&E stains were reviewed to characterize lesion type, growth pattern (infiltrative vs circumscribed), presence of squamous metaplasia, spindle cell nuclear atypia and mitoses/10HPF and biopsy site changes, if present. Cases were immunostained for cytokeratins (CK5/6, CK903, MNF116) and p63. The extent (focal/diffuse) and intensity (weak/moderate/strong) of positive staining within the BSL and biopsy site were evaluated.

Results: Thirty-two CSL/RS and $23 \mathrm{ScPap}$ lesions were evaluated. Immunostain results are shown in the Table.

\begin{tabular}{|c|c|c|c|c|c|}
\hline & & $7 \overline{C K ~ 5 / 6}$ & CK 903 & MNF116 & $\mathrm{p} 63$ \\
\hline \multirow[t]{2}{*}{$\overline{\mathrm{CSL} / \mathrm{RS}}$} & Bx Site & $0 / 8$ & $0 / 9$ & $1 / 10(10 \%)$ & $0 / 9$ \\
\hline & Lesion & $0 / 29$ & $0 / 29$ & $7 / 30(23 \%)$ & $1 / 27(4 \%)$ \\
\hline \multirow[t]{2}{*}{ ScPap } & Bx Site & $0 / 15$ & $0 / 15$ & $1 / 15(6 \%)$ & $0 / 15$ \\
\hline & Lesion & $1 / 22(5 \%)$ & $1 / 21(5 \%)$ & $3 / 23(13 \%)$ & $0 / 22$ \\
\hline \multirow[t]{2}{*}{ Overall } & Bx Site & $0 / 23$ & $0 / 24$ & $2 / 25(8 \%)$ & $0 / 24$ \\
\hline & Lesion & $1 / 51(2 \%)$ & $1 / 50(2 \%)$ & $10 / 53(19 \%)$ & $1 / 51(2 \%)$ \\
\hline
\end{tabular}

\# positive cases/total evaluable cases

Among 55 cases, none showed spindle cell reactivity for all 4 markers; 1 case (2\%) showed positivity for 3 markers (CK5/6, CK903, MNF116); 1 case $(2 \%)$ for 2 markers (MNF116, p63); 8 cases (15\%) for 1 marker (all MNF116). Most positive cases demonstrated very focal, weak staining of spindle cells with just 1 case showing moderate staining with all CKs. Spindle cell positivity was not associated with lesion type, growth pattern, spindle cell atypia or mitoses, nor with development of recurrent lesions.

Conclusions: Stromal cells of $81 \%$ of BSLs showed no staining for CKs or p63. Among the remaining cases, most showed only focal, weak staining, most often with MNF116. These findings suggest that while expression of CK5/6 and CK 903 may be useful tools to distinguish BSLs from metaplastic carcinomas arising in this setting, MNF116 staining should be interpreted with caution.

281 If Re-Excision Is Necessary When Invasive Mammary Carcinoma Is within $1 \mathrm{~mm}$ to Inked Margin and If Tumor Focality or Ductal or Lobular Plays a Role on Residual Tumor

$R$ Omman, $S$ Al Diffalha, S Pambuccian, Y Li, C Ersahin, C Perez, C Godellas, $R$ Gamez, X Duan. Loyola University Medical Center, Maywood, IL.

Background: A common problem with breast conserving surgery is the question of a safe margin, i.e what is the margin with no need for re-excision? Majority agree that tumor on ink is valid for re-excision but no consensus has come as far as close margins. Much controversy has surrounded this issue and different institutions and surgeons have different opinion as to when to re-excise. In this study, we will examine the rate of residual tumor on re-excision for positive and close margins; compare the difference on residual tumor between ductal and lobular carcinoma and between multifocal and single focus tumors.

Design: We searched our database from 2005-2012 and identified a total of 500 invasive ductal carcinoma and 72 invasive lobular carcinoma lumpectomy specimens. We then examined 92 invasive breast cancers treated with re-excision with margins either positive (on ink) or close (within $1 \mathrm{~mm}$ of inked margin). We examined the percentage of tumor identified on re-excision and compared the difference of residual tumors between ductal and lobular carcinoma and between multifocal and single focus of tumor

Results:

Comparison of positive tumors on re-excision between tumor at ink and tumor within $1 \mathrm{~mm}$ and

\begin{tabular}{|l|l|l|l|l||}
\hline Initial Lumpectomy & Total tumor at ink: 56 & Total tumor within 1 mm: 36 \\
\hline \hline \# & Ductal: 47 & Lobular: 9 & Ductal: 30 & Lobular:6 \\
\hline \# of positive cases on re-excision & 19 & 7 & 5 \\
\hline \% of positive cases on re-excision & $40 \%$ & $77 \%$ & $16 \%$ \\
\hline Total positive & $26(46 \%)$ & $8 \%$ \\
\hline
\end{tabular}

Total positive

We also examined if multifocality played a role on residual tumor on re-excision. We identified 18 total multifocal tumors on initial excision of which 11 were at ink and 7 were within $1 \mathrm{~mm}$. 10/11 at ink multifocal tumors were positive for residual tumor on re-excision, while only $2 / 7$ cases within $1 \mathrm{~mm}$ had residual tumor present on re-excision. Conclusions: The percentage of residual tumors were $46 \%$ and $22 \%$ on re-excision for original tumor with positive and close margins respectively. We also found that there was a higher percentage of residual tumor in lobular vs ductal carcinoma. Also, a majority of multifocal tumors at ink continued to persist on re-excision when compared to multifocal tumors within $1 \mathrm{~mm}$. Tumor within $1 \mathrm{~mm}$ to inked margin needs to be re-excised.

282 Does a Standardized Protocol for Measuring Ki67 Improve Interobserver Variability in Breast Carcinoma?

A Ordobazari, J Stone, B Hunt, B Behmaram. MCW, Milwaukee, WI.

Background: Use of ki67 labeling index (LI) has gained significant popularity as a proposed prognostic marker in breast carcinoma biopsy. Very high or low values of Ki67 labeling correlate well with histologic grade and are otherwise not problematic. However, the accuracy and precision of the clinically relevant intermediate values are less clear. This study was designed to determine if a standardized method of estimating Ki67 LI would provide increased interobserver agreement.

Design: 100 biopsies of invasive mammary carcinoma were reviewed. 13 were eliminated due to inadequate material. MIB-1 immunostain on the 87 core needle biopsies, reviewed by 3 pathologists and a resident using 2 different methods: 1) Visually estimating the percentage of positive staining malignant cells; 2) Calculating 
an estimate of positive staining malignant cells on three representative $40 \mathrm{X}$ fields using a 10x10 square grid eyepiece graticule with a standardized method. All 87 cases were evaluated with visual estimates. Only the 26 grade 2 cases evaluated by calculation due to high agreement seen with low grade and high grade lesions. The agreement between the estimates of readers was assessed with Cohen's kappa and intraclass correlation coefficient (ICC).

Results: The percentage of positive cells grouped into 3 categories: $\leq 5 \%$ (kappa:0.69), $6-19 \%$ (kappa:0.40), $\geq 20 \%$ (kappa:0.74), for overall kappa of 0.62 . For samples scored within the $6-19 \%$ range, numeric agreement was evaluated using ICC; grade 1: 0.27 , grade 2: 0.14 , overall: 0.24 . The resident data was consistently an outlier with higher estimation, and recalculation with 3 pathologists gave an overall kappa of 0.76 . The standardized method of calculating grade 2 lesions had an overall ICC: 0.65 vs ICC: 0.53 from visual estimates. Regarding the $6-19 \%$ group, overall ICC for grade 2 was 0.18 , with visual estimation of 0.14 .

Conclusions: The common practice of estimating Ki67 LI can lead to inaccurate evaluations, usually in the direction of higher index, particularly among less experienced pathologists. This is especially problematic in grade 2 carcinomas where clinical decisions can be influenced by Ki67 LI. Our study shows that in grade 2 disease, there was only fair correlation in estimating Ki67 LI which was minimally improved by using a standardized method. The differences observed when using a standardized method are likely due to variability in $40 \mathrm{X}$ field selection and variable interpretation of other cell types such as lymphocytes.

\section{The Role of Notch Signaling in the Regulation of FOXP3 Expression} in Breast Carcinoma

F Ortiz-Martinez, FJ Gutierrez-Avino, A Perez-Balaguer, P Martinez-Peinado, M Rodrigo, FI Aranda, E Lerma, J Sanchez-Paya, JM Sempere-Ortells, G Peiro. University General Hospital, Alacant, Spain; University of Alacant, Alacant, Spain; Hospital de la Santa Creu i Sant Pau, Barcelona, Spain.

Background: Notch pathway has a role in normal cell growth and development. Its activation has been reported in several neoplasms, especially those with positive hormonal receptor status. Experimental studies also suggest its potential role in the regulation of FOXP3 expression and Tregs differentiation and expansion. In breast carcinoma (BC), the relationship between Notch signaling and FOXP3 expression is unknown.

Design: We analyzed by quantitative RT-PCR the mRNA expression of Notch1, Notch2, Notch4 and FOXP3 in a series of 152 BC patients (74 Luminal and 78 Triple-negative/ Basal-like [TN/BL]). Relative mRNA expression levels were carried out using the $\Delta \Delta \mathrm{C}_{\mathrm{T}}$ method and they were expressed as the fold-change (FC). A pool of normal breast tissues was used as calibrator for Luminal BC, whereas TN/BL tumors were compared with the adjacent tumor tissue. FOXP3+ Tregs within the tumor and/or immediately adjacent stroma were counted in 3 high power fields ( $\mathrm{x} 400$ ). The mRNA expression levels, Tregs tumor content and clinical-pathological factors were correlated.

Results: High median FOXP3 mRNA expression was found among tumors $>20$ $\mathrm{mm}(\mathrm{p}=0.026)$, grade $3(\mathrm{p}=0.006)$, positive lymph-node status $(\mathrm{p}=0.026)$ and among tumors with high number of Tregs (median threshold $\geq 15$ FOXP3+ cells) as a trend $(\mathrm{p}>0.05)$. In contrast, higher median of Notch1, Notch2 and Notch4 mRNA expression were found among tumors $\leq 20 \mathrm{~mm}$ (all $\mathrm{p}<0.019$ ), Luminal phenotype (all $\mathrm{p}<0.019$ ), negative lymph-node status (all $\mathrm{p} \leq 0.023$ ), and as a trend in those tumors with low Tregs content (all $\mathrm{p} \leq 0.140$; Mann-Whitney/Kruskal-Wallis tests). Moreover, Notch2 mRNA expression was negatively correlated with FOXP3 mRNA expression ( $\mathrm{r}=-0.188$, $\mathrm{p}=0.020$; Spearman's Rank Correlation)

Conclusions: Our findings in a series of $\mathrm{BC}$ indicate that Notch signaling is activated in Luminal tumors with good prognostic factors. In contrast, this pathway, and specifically Notch2, negatively modulates FOXP3 expression and Tregs content in TN/BL phenotype.

Supported by Grants FIS 10/00082, AP-172/10, FCVI-HGUA (2009/GE02B and 2011/PC-03)

\section{CD44 Induces FOXP3 Expression in Regulatory T-Cells (Tregs) in Triple Negative/Basal-Like Breast Carcinoma}

F Ortiz-Martinez, A Perez-Balaguer, P Martinez-Peinado, E Lerma, JM SempereOrtells, FI Aranda, G Peiro. University General Hospital, Alacant, Spain; University of Alacant, Alacant, Spain; Hospital de la Santa Creu i Sant Pau, Barcelona, Spain. Background: FOXP3 (Xp11.23) has been identified as a tumor suppressor gene that regulates tumor development. Its product is an essential transcription factor for immune suppressive function of regulatory T-cells (Tregs). Recent research suggests that stimulation of the receptor CD44, a cancer stem cell marker, induces FOXP3 expression in Tregs. However, the relationship between CD44 and FOXP3 in breast cancer $(\mathrm{BC})$ is unknown.

Design: We evaluated FOXP3 and CD44 mRNA expression by qRT-PCR using TaqMan assays in breast cancer cell lines (MCF7, BT474, SKBR3, MDAMB231 and MDAMB468) and in 154 primaries BC. Relative changes in gene expression were calculated as the fold-change (FC) by the $2^{-\Delta \Delta C t}$ method. PUM1 and ACTB were the reference genes. Normal epithelial breast cell line 184A1 and breast tissue were used as control samples for cells and carcinomas, respectively. FOXP3+ Tregs within the tumor and/or immediately adjacent stroma were counted in 3 high power fields ( $x 400)$. BCs were classified immunohistochemically into Luminal (27\%), HER2+ $(35 \%)$ or Triple Negative/Basal-Like (TN/BL) (38\%)

Results: MCF7, BT474 and SKBR3 cell lines overexpressed FOXP3 but not CD44, whereas MDAMB231 and MDAMB468 showed the opposite results. High median FOXP3 mRNA expression was seen in grade 3 tumors $(\mathrm{p}=0.014)$ with high Tregs content (median threshold $>15$ FOXP3 + cells $)(F C=4.16, p=0.040)$. High median CD44 mRNA expression was observed in tumors of older patients ( $\mathrm{p}=0.028$; Mann-Whitney
U and Kruskal-Wallis tests). A positive correlation between FOXP3 and CD44 mRNA expression was detected only in TN/BL tumors ( $r=0.502, \mathrm{p}<0.001$; Spearman's Rank Correlation).

Conclusions: Our results in a series of BC suggest that CD44 induces FOXP3 expression in Tregs, which are increased in TN/BL phenotype.

Supported by Grants FIS 10/00082, AP-172/2010, FCVI-HGUA (2010/PC-04 and 2011/PC-03).

285 A Positive Correlation between Osteopontin, VEGFA and ICAM1 mRNA Expression in Breast Carcinoma: Treatment and Prognostic Implications

F Ortiz-Martinez, A Perez-Balaguer, J Sanchez-Paya, FI Aranda, E Lerma, G Peiro. University General Hospital, Alacant, Spain; Hospital de la Santa Creu i Sant Pau, Barcelona, Spain.

Background: $S P P 1$ (secreted phosphoprotein 1) is a metastasis-associated gene that encodes osteopontin (OPN), a glycophosphoprotein secreted by multiple human tissues. Experimental studies in breast cancer (BC) cells and in vivo studies with mice support that OPN induces the expression of cancer biomarkers involved in mechanisms of cell survival (Bcl2), invasiveness (ICAM-1) and angiogenesis (VEGFA). However, this has not been confirmed in clinical series of $\mathrm{BC}$ yet.

Design: 148 BC tumors were classified immunohistochemically into Luminal (32\%), HER2-positive $(30 \%)$ and Triple Negative/Basal-Like (TN/BL; 38\%) subtypes. Quantitative real-time PCR was performed using TaqMan Assays to analyze >BCL2, $>$ ICAM1, $>$ PUM1, $>$ SPP1 and $>$ VEGFA gene expression. The $2^{-\Delta \Delta C t}$ method was used to calculate the mRNA expression as the fold change (FC). Normal mammary tissue was used as the calibrator sample and $>$ PUM1 as the reference gene.

Results: The median of $\mathrm{Bcl} 2 \mathrm{mRNAFC}$ was higher among tumors with low histological grade (1 and 2 ) and Luminal phenotype. In contrast, the median of ICAM-1 and VEGFA mRNA FC was higher among TN/BL phenotype. Moreover, higher VEGFA mRNA levels were found among tumors $>20 \mathrm{~mm}$ (all $\mathrm{p}<0.05$; Mann-Whitney $U$ and KruskalWallis tests). OPN mRNA expression correlated positively with ICAM-1 ( $\mathrm{r}=0.225$, $\mathrm{p}=0.006$ ) and VEGFA mRNA levels ( $\mathrm{r}=0.283, \mathrm{p}<0.000$; Spearman's Rank Correlation). Survival analysis showed that in patients with OPN overexpressing tumors, VEGFA mRNA level remained as an independent prognostic factor for recurrence $(\mathrm{HR}=2.95$, 95\% CI: $1.48-5.87, \mathrm{p}=0.002)$ and death $(\mathrm{HR}=3.25,95 \% \mathrm{CI}: 1.48-7.11, \mathrm{p}=0.003)$ (Multivariate analysis; Cox regression).

Conclusions: Our study provides further insight into the mechanism of action of OPN through the regulation of invasion (ICAM-1) and angiogenesis (VEGFA) pathways, especially in TN/BL BC. In addition, the demonstration of an independent prognostic value of VEGFA makes it a potential target for antiangiogenic therapy in this aggressive phenotype.

Supported by Grants FIS 10/00082, FCVI-HGUA (NI-04/2011, E-02/2012).

286 Routine Ancillary Studies Accurately Are Correlated with Oncotype DX Recurrence Score

$R$ Owings, E Gould, A Renshaw. Brigham and Women's Hospital, Boston, MA; Baptist Hospital of Miami, Miami, FL.

Background: Oncotype $\mathrm{DX}^{\mathrm{TM}}$ quantifies the risk of recurrence in ER positive, Stage 1 or 2 breast cancer patients treated with tamoxifen and also helps assess the added benefit of chemotherapy. Many oncologists find the results useful to guide therapy, but some patients are not tested, due to financial or access concerns. Several studies have shown that Oncotype DX recurrence score (RS) is correlated with routine ancillary studies. The study aimed to develop a model that would divide cancers into those for which RS could be predicted and those for which RS may provide additional information. Design: The model was originally developed using 39 cases and then validated and revised after testing an additional 32 cases, using cases sent for Oncotype DX testing. The factors were ER and PR (percentage and intensity), HER2, mitotic score (modified Bloom Richardson score, 1 to 3 ) and histologic type (mucinous or nonmucinous). The resulting algorithm is shown in Figure 1. The model was designed to predict cases that were low risk or high risk. All other cases were considered intermediate/unclear risk that might benefit from Oncotype DX testing. Additional consecutive cases from a second hospital were used to test the model.

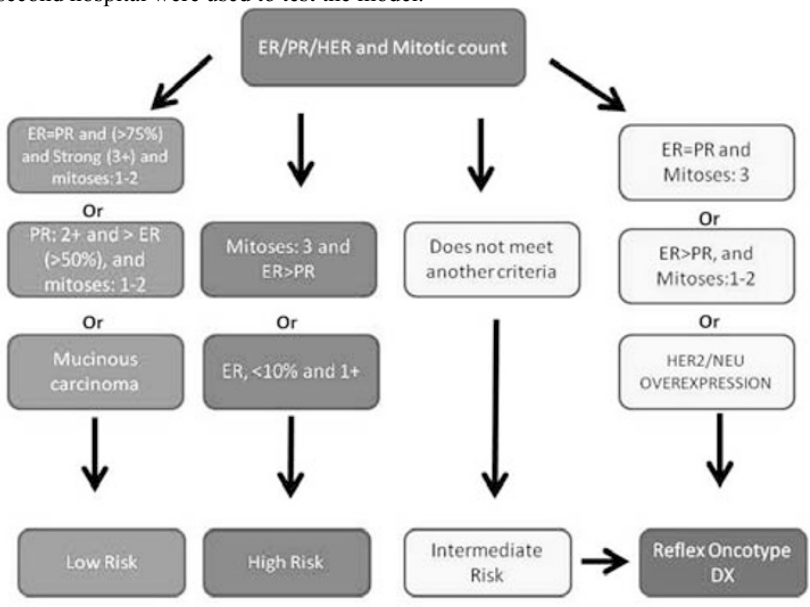


Results: From 237 cases, the algorithm correctly classified $223(94 \%)$ to the appropriate RS groups by direct classification or by suggesting an Oncotype DX test. Of these cases, $166(70 \%)$ were correctly assigned to an RS category, while only $112(47 \%)$ required reflex to Oncotype, a reduction of $53 \%$ (correctly classified intermediate risk cases were reflexed). The algorithm classified 113 cases as low risk (1 reflexed), 107 cases as intermediate risk (all reflexed), and 17 cases as high risk (4 reflexed). 14 (6\%) cases were incorrectly classified and not reflexed to Oncotype. 13 of these were classified as low risk and 1 as high risk, while RS assigned them to intermediate risk.

Conclusions: Routine diagnostic information can predict RS classification in a subset of cancer cases. When reflexed to Oncotype DX for intermediate cases, it can achieve similar classification in $94 \%$, while reducing Oncotypes $53 \%$. The use of such an algorithm could help guide therapy and reduce the need for some Oncotype DX testing in certain settings.

\section{Claudin Profile of Molecular and Morphological Subtypes of Breast} Cancer

U Ozerdem, Y Liu, TM D'Alfonso, SJ Shin. Weill Cornell Medical College, New York, NY.

Background: Claudins contribute to cohesion of epithelial cells. Herein, we set out to elucidate the expression of Claudin 1, 3, 4, and 7 in subtypes of breast cancer

Design: Tissue microarrays containing Luminal A ( $\mathrm{n}=150)$, HER2 ( $\mathrm{n}=103)$, Luminal B $(\mathrm{ER}+/ \mathrm{PR}+/ \mathrm{HER} 2+, \mathrm{n}=106)$, Luminal $\mathrm{B}(\mathrm{ER}+/ \mathrm{PR}+/ \mathrm{Ki} 67>14 \%, \mathrm{n}=100)$, triple negative $(\mathrm{n}=149)$, DCIS $(\mathrm{n}=45)$, LCIS $(\mathrm{n}=21)$, and infiltrating lobular carcinoma (IFLC, $\mathrm{n}=48)$ were immunostained. Slides were digitized with Aperio. Image analysis was performed with NIH ImageJ to calculate an integrated density. Data were analyzed using two-way ANOVA and Bonferroni post-tests.

Results: There was a significant difference of Claudin 1, 3, 4 and 7 expression between subtypes of breast cancer $(\mathrm{P}<0.0001)$.

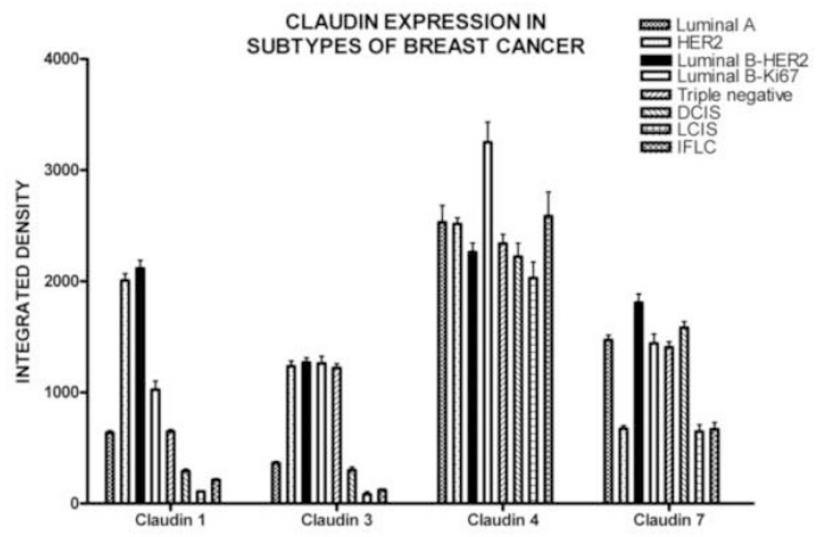

RESULTS OF POST-HOC STATISTICAL COMPARISONS WITH BONFERRONI POSTTESTS

\begin{tabular}{|c|c|c|c|c|}
\hline & Claudin 1 & Claudin 3 & Claudin 4 & Claudin 7 \\
\hline Luminal A vs. HER2 & $\mathrm{P}<0.001$ & $\mathrm{P}<0.001$ & $\mathrm{P}>0.05$ & $\mathrm{P}<0.001$ \\
\hline Luminal A vs. Luminal B-HER2 & $\mathrm{P}<0.001$ & $\mathrm{P}<0.001$ & $\mathrm{P}>0.05$ & $\mathrm{P}<0.05$ \\
\hline Luminal A vs. Luminal B-Ki67 & $\mathrm{P}<0.01$ & $\mathrm{P}<0.001$ & $\mathrm{P}<0.001$ & $\mathrm{P}>0.05$ \\
\hline Luminal A vs. Triple negative & $P>0.05$ & $\mathrm{P}<0.001$ & $\mathrm{P}>0.05$ & $\mathrm{P}>0.05$ \\
\hline Luminal A vs. DCIS & $\mathrm{P}<0.01$ & $\mathrm{P}>0.05$ & $\mathrm{P}<0.01$ & $\mathrm{P}>0.05$ \\
\hline Luminal A vs. LCIS & $\mathrm{P}<0.001$ & $\mathrm{P}<0.05$ & $\mathrm{P}<0.001$ & $\mathrm{P}<0.001$ \\
\hline Luminal A vs. IFLC & $\mathrm{P}<0.001$ & $P>0.05$ & $\mathrm{P}>0.05$ & $\mathrm{P}<0.001$ \\
\hline HER2 vs. Luminal B-HER2 & $\mathrm{P}>0.05$ & $\mathrm{P}>0.05$ & $\mathrm{P}>0.05$ & $\mathrm{P}<0.001$ \\
\hline HER2 vs. Luminal B-Ki67 & $\mathrm{P}<0.001$ & $\mathrm{P}>0.05$ & $\mathrm{P}<0.001$ & $\mathrm{P}<0.001$ \\
\hline HER2 vs. Triple negative & $\mathrm{P}<0.001$ & $\mathrm{P}>0.05$ & $\mathrm{P}>0.05$ & $\mathrm{P}<0.001$ \\
\hline HER2 vs. DCIS & $\mathrm{P}<0.001$ & $\mathrm{P}<0.001$ & $\mathrm{P}<0.05$ & $\mathrm{P}<0.001$ \\
\hline HER2 vs. LCIS & $\mathrm{P}<0.001$ & $\mathrm{P}<0.001$ & $\mathrm{P}<0.001$ & $\mathrm{P}>0.05$ \\
\hline HER2 vs. IFLC & $\mathrm{P}<0.001$ & $\mathrm{P}<0.001$ & $\mathrm{P}>0.05$ & $\mathrm{P}>0.05$ \\
\hline Luminal B-HER2 vs. Luminal B-Ki67 & $\mathrm{P}<0.001$ & $\mathrm{P}>0.05$ & $\mathrm{P}<0.001$ & $\mathrm{P}>0.05$ \\
\hline Luminal B-HER2 vs. Triple negative & $\mathrm{P}<0.001$ & $\mathrm{P}>0.05$ & $\mathrm{P}>0.05$ & $\mathrm{P}<0.01$ \\
\hline Luminal B-HER2 vs. DCIS & $\mathrm{P}<0.001$ & $\mathrm{P}<0.001$ & $P>0.05$ & $\mathrm{P}>0.05$ \\
\hline Luminal B-HER2 vs. LCIS & $\mathrm{P}<0.001$ & $\mathrm{P}<0.001$ & $\mathrm{P}>0.05$ & $\mathrm{P}<0.001$ \\
\hline Luminal B-HER2 vs. IFLC & $\mathrm{P}<0.001$ & $\mathrm{P}<0.001$ & $\mathrm{P}>0.05$ & $\mathrm{P}<0.001$ \\
\hline Luminal B-Ki67 vs. Triple negative & $\mathrm{P}<0.01$ & $\mathrm{P}>0.05$ & $\mathrm{P}<0.001$ & $\mathrm{P}>0.05$ \\
\hline Luminal B-Ki67 vs. DCIS & $\mathrm{P}<0.001$ & $\mathrm{P}<0.001$ & $\mathrm{P}<0.001$ & $\mathrm{P}>0.05$ \\
\hline Luminal B-Ki67 vs. LCIS & $\mathrm{P}<0.001$ & $\mathrm{P}<0.001$ & $\mathrm{P}<0.001$ & $\mathrm{P}<0.001$ \\
\hline Luminal B-Ki67 vs. IFLC & $\mathrm{P}<0.001$ & $\mathrm{P}<0.001$ & $\mathrm{P}<0.001$ & $\mathrm{P}<0.001$ \\
\hline Triple negative vs. DCIS & $\mathrm{P}<0.01$ & $\mathrm{P}<0.001$ & $\mathrm{P}>0.05$ & $\mathrm{P}>0.05$ \\
\hline Triple negative vs. LCIS & $\mathrm{P}<0.001$ & $\mathrm{P}<0.001$ & $\mathrm{P}<0.05$ & $\mathrm{P}<0.001$ \\
\hline Triple negative vs. IFLC & $\mathrm{P}<0.001$ & $\mathrm{P}<0.001$ & $\mathrm{P}>0.05$ & $\mathrm{P}<0.001$ \\
\hline DCIS vs. LCIS & $\mathrm{P}>0.05$ & $\mathrm{P}>0.05$ & $\mathrm{P}>0.05$ & $\mathrm{P}<0.001$ \\
\hline DCIS vs. IFLC & $\mathrm{P}>0.05$ & $\mathrm{P}>0.05$ & $\mathrm{P}<0.01$ & $\mathrm{P}<0.001$ \\
\hline LCIS vs. IFLC & $\mathrm{P}>0.05$ & $\mathrm{P}>0.05$ & $\mathrm{P}<0.001$ & $\mathrm{P}>0.05$ \\
\hline
\end{tabular}

Claudin 1 was overexpressed in HER2 and Luminal B-HER2 subtypes, and down regulated in LCIS and IFLC. Claudin 4 expression in all subtypes was higher than Claudin 1,3, and 7 expression.

Conclusions: Loss of Claudin 1 and 3 in LCIS and IFLC could be exploited as an adjunct diagnostic tool.
288 VEGF Receptor-3 Expression in Molecular and Morphological Subtypes of Breast Cancer

U Ozerdem, Y Liu, TM D'Alfonso, SJ Shin. Weill Cornell Medical College, New York, NY.

Background: It is imperative to identify molecular targets in a given breast specimen to personalize novel targeted therapies such as anti-VEGFR-3 monoclonal antibodies. Herein, we set out to elucidate the VEGFR-3 expression in subtypes of breast cancer. Design: Tissue microarrays containing Luminal A (ER+/PR+/HER2-, n=150), HER2 (ER-/PR-/HER2+, n=103), Luminal B (ER+/PR+/HER2+, n=106), Luminal B (ER+/ PR $+/$ HER2-/Ki67 $>14 \%, n=100$ ), triple negative (ER-,PR-,HER2-, $n=149$ ), ductal carcinoma in situ (DCIS, $\mathrm{n}=45$ ), lobular carcinoma in situ (LCIS, $\mathrm{n}=21$ ), and infiltrating lobular carcinoma (IFLC $\mathrm{n}=48$ ) were immunostained for VEGFR-3. Slides were digitized with Aperio Scanscope. Image analysis of digitized slides were performed by using NIH ImageJ software to calculate an integrated density (intensity $\mathrm{x}$ area) of VEGFR-3. Data were analyzed with Prism software using one-way ANOVA and Bonferroni post hoc test.

Results: VEGFR-3 was expressed by breast cancer cells and tumor vasculature. There is a statistically significant difference of VEGFR 3 expression between molecular subtypes of breast cancer $(\mathrm{P}<0.0001)$.

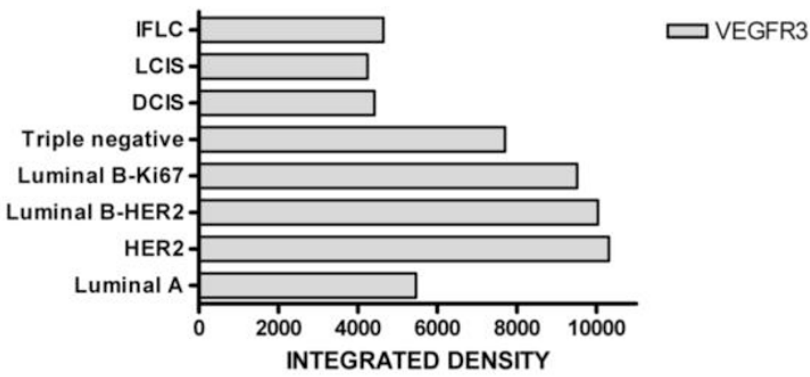

BONFERRONI MULTIPLE COMPARISON TEST FOR VEGFR3 EXPRESSION IN BREAST

\begin{tabular}{|c|c|}
\hline & \\
\hline Luminal A vs. HER2 & $\mathrm{P}<0.001$ \\
\hline Luminal A vs. Luminal B-HER2 & $\mathrm{P}<0.001$ \\
\hline Luminal A vs. Luminal B-Ki67 & $\mathrm{P}<0.001$ \\
\hline Luminal A vs. Triple negative & $\mathrm{P}<0.001$ \\
\hline Luminal A vs. DCIS & $\mathrm{P}>0.05$ \\
\hline Luminal A vs. LCIS & $\mathrm{P}>0.05$ \\
\hline Luminal A vs. IFLC & $\mathrm{P}>0.05$ \\
\hline HER2 vs. Luminal B-HER2 & $\mathrm{P}>0.05$ \\
\hline HER2 vs. Luminal B-Ki67 & $\mathrm{P}>0.05$ \\
\hline HER2 vs. Triple negative & $\mathrm{P}<0.001$ \\
\hline HER2 vs. DCIS & $\mathrm{P}<0.001$ \\
\hline HER2 vs. LCIS & $\mathrm{P}<0.001$ \\
\hline HER2 vs. IFLC & $\mathrm{P}<0.001$ \\
\hline Luminal B-HER2 vs. Luminal B-Ki67 & $\mathrm{P}>0.05$ \\
\hline Luminal B-HER2 vs. Triple negative & $\mathrm{P}<0.001$ \\
\hline Luminal B-HER2 vs. DCIS & $\mathrm{P}<0.001$ \\
\hline Luminal B-HER2 vs. LCIS & $\mathrm{P}<0.001$ \\
\hline Luminal B-HER2 vs. IFLC & $\mathrm{P}<0.001$ \\
\hline Luminal B-Ki67 vs. Triple negative & $\mathrm{P}<0.05$ \\
\hline Luminal B-Ki67 vs. DCIS & $\mathrm{P}<0.001$ \\
\hline Luminal B-Ki67 vs. LCIS & $\mathrm{P}<0.001$ \\
\hline Luminal B-Ki67 vs. IFLC & $\mathrm{P}<0.001$ \\
\hline Triple negative vs. DCIS & $\mathrm{P}<0.001$ \\
\hline Triple negative vs. LCIS & $\mathrm{P}<0.001$ \\
\hline Triple negative vs. IFLC & $\mathrm{P}<0.001$ \\
\hline DCIS vs. LCIS & $\mathrm{P}>0.05$ \\
\hline DCIS vs. IFLC & $\mathrm{P}>0.05$ \\
\hline LCIS vs. IFLC & $\mathrm{P}>0.05$ \\
\hline
\end{tabular}

Conclusions: VEGFR3 is expressed by tumor cells and vasculature of breast cancer. VEGFR3 expression was higher in HER2, Luminal B and triple negative subtypes than Luminal A, IFLC, LCIS and DCIS. Differences of VEGFR3 expression in subtypes of breast cancer could be exploited for therapeutic targeting using anti-VEGFR-3 monoclonal antibodies.

289 Metaplastic Carcinoma of Breast: A Clinicopathologic Study of 94 Cases

A Patil, P Motghare, T Shet, S Desai. Tata Memorial Hospital, Mumbai, Maharashtra, India.

Background: Metaplastic carcinoma of breast (MCB) comprises of a heterogeneous group of breast carcinoma showing sqaumous and/or mesenchymal differentiation and are characterized by overall worse clinical outcome with lower response to adjuvant chemotherapy. Individual subtypes of MCB have been shown to be associated with variable prognosis. The aim of this study was to evaluate clinical and pathological characteristics of different subtypes of MCB.

Design: We identified 94 cases of MCB from pathology records between years 20032010 with slides and blocks available for study. The morphological features were assessed by two pathologists (AP and PM) and the cases were divided into three categories: Sqaumous (SC), sarcomatoid (SR) and matrix producing (MP) types. SR cases without overt epithelial component were confirmed by expression of one or more epithelial markers (AE1/AE3, CK5/6, CK14, CK17). Clinical data was obtained from electronic medical records. 
Results: All 94 patients were females (age: 21-83 yr, median 50 yr). 59 cases were treated at our institution, while 35 were referral cases with limited data available. Tumor size in 59 cases ranged from $1-25 \mathrm{~cm}$ (median $5 \mathrm{~cm}$ ), with IIA as most common stage of presentation. 38 of 53 sarcomatoid carcinoma cases showed accompanying epithelial component with 10 also showing DCIS, while 15 cases showed pure spindle cell morphology with expression of epithelial markers. Commonest nuclear grade was 3 ( 88 cases, $94 \%$ ), followed by 2 ( 6 cases, $6 \%$ ). Heterologous component was noted in 19 cases $(20 \%)$, commonest in SR (16 cases). The clinicopathological characteristics are described in Table 1. Lymph node metastases, more common in SC, were also seen in SR, including 4 pure SR cases without overt epithelial component. We did not find any significant association of three subtypes with overall survival (OS) and disease free survival (DFS).

Table 1. Clinicopathological findings in MCB ( $\mathrm{n}=94)$
\begin{tabular}{|l|l|l|l||l|}
\hline \hline & Sqaumous & Sarcomatoid & Matrix producing & Total \\
\hline Total number of cases & $24 / 94(26 \%)$ & $53 / 94(56 \%)$ & $17 / 94(18 \%)$ & 94 \\
\hline DCIS & $9 / 24(37 \%)$ & $10 / 53(19 \%)$ & $0 / 17(0 \%)$ & $19 / 94(20 \%)$ \\
\hline Lymphovascular emboli (LVI) & $6 / 24(25 \%)$ & $3 / 53(6 \%)$ & $1 / 17(6 \%)$ & $10 / 94(11 \%)$ \\
\hline Lymph node metastasis & $10 / 16(63 \%)$ & $14 / 32(44 \%)$ & $6 / 12(50 \%)$ & $30 / 60(50 \%)$ \\
\hline Local recurrence & $4 / 14(29 \%)$ & $14 / 30(47 \%)$ & $2 / 10(20 \%)$ & $20 / 54(37 \%)$ \\
\hline Distant metastasis & $2 / 18(11 \%)$ & $10 / 35(29 \%)$ & $2 / 12(17 \%)$ & $14 / 65(22 \%)$ \\
\hline
\end{tabular}

Conclusions: In our series of MCB, SR was the commonest subtype, with more frequent local recurrence and distant metastasis, while DCIS, LVI and nodal metastasis was more commonly seen in SC. Fnding nodal metastasis in pure SR emphasizes need of axillary dissection in these cases.

\section{Lobular Neoplasia within a Fibroadenoma: The Decision for} Excision

L Pelaez, S Alghamdi, RJ Poppiti. Mount Sinai Medical Center, Miami Beach, FL. Background: Fibroadenomas are the most common benign tumor in the female breast. Epithelial proliferative changes may occur in a fibroadenoma, including lobular neoplasia. Infiltrating carcinoma may also involve a fibroadenoma. It has been reported that "in situ" carcinomas when present in a fibroadenoma are confined to the fibroadenoma in the majority of cases. The aim of our study is to evaluate the association between fibroadenomas harboring lobular neoplasia and the presence of lobular neoplasia and infiltrating carcinoma in the surrounding breast tissue.

Design: We retrospectively reviewed our pathology archives for fibroadenomas diagnosed between 2000 and 2013. The cases of lobular neoplasia within fibroadenomas were identified. Demographics, radiological findings and associated histopathologic diagnoses, along with surgical follow up data were analyzed.

Results: A total of 3,491 women were diagnosed with fibroadenomas in our institution from 2000 to 2013, fourteen of which harbored lobular neoplasia within their fibroadenomas. The median age was 58 years (range: $42-81$ ). The most common radiological finding was the presence of a mass $(8 / 14)$, followed up by microcalcifications (5/14). All of the patients underwent image-guided core biopsy. Ten fibroadenomas were hyalinized, three of which had calcifications. Ten of the fourteen women had lobular neoplasia in the surrounding breast tissue, and in two of them lobular neoplasia was also present on the contralateral breast. Six of the fourteen women had infiltrating carcinoma, four of which were of the duct cell type and two were lobular. Two women had infiltrating ductal carcinoma in the core biopsy and subsequently showed lobular neoplasia within fibroadenomas in the contralateral excision specimens. Associated Histopathologic Findings Present in Women with Fibroadenoma with Lobular

\begin{tabular}{|c|c|c|c|c|}
\hline Case & Age & $\begin{array}{l}\text { Lobular Neoplasia } \\
\text { Outside Fibroadenoma }\end{array}$ & Infiltrating Carcinoma & Hyalinization of Fibroadenoma \\
\hline 1 & 76 & 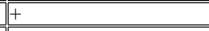 & Lobular type & + \\
\hline 2 & 73 & + & - & + \\
\hline 3 & 68 & + & Ductal type & + \\
\hline 4 & 50 & - & - & + \\
\hline 5 & 45 & + & Lobular type & + \\
\hline 6 & 81 & + & - & + \\
\hline 7 & 68 & + & Ductal type & - \\
\hline 8 & 51 & - & - & - \\
\hline 9 & 74 & + & Ductal type & + \\
\hline 10 & 64 & + & - & + \\
\hline 11 & 68 & + & - & - \\
\hline 12 & 69 & + & Ductal type & + \\
\hline 13 & 42 & - & - & + \\
\hline 14 & 43 & - & - & - \\
\hline
\end{tabular}

Conclusions: Seventy one percent of the women diagnosed with fibroadenoma harboring lobular neoplasia, had at least lobular neoplasia outside of the fibroadenoma. Forty three percent of our patients had infiltrating carcinoma. Although previous reports have stated that fibroadenoma with lobular neoplasia may be an isolated event, our study showed that this is rarely the case, and thus local excision, at least, should be the treatment of choice.

291 Changes in the Immunohistochemically-Defined Molecular Subtypes of Breast Cancer during Neoadjuvant Chemotherapy: Impact on Prognosis of $\mathbf{2 8 2}$ Patients in a 10-Year Follow-Up Study

F Penault-Llorca, CAbrial, Q Wang-Lopez, M-M Dauplat, A Cayre, NRadosevic-Robin, M-A Mouret-Reynier, P Chollet, J-M Nabholtz. Jean Perrin Cancer Center and ERTICa Research Group, University of Auvergne, Clermont-Ferrand, France; Jean Perrin Cancer Center and INSERM UMR990, University of Auvergne, Clermont-Ferrand, France. Background: Neoadjuvant chemotherapy (NACT) for breast cancer (BC) is aimed to achieve a pathologic complete response (pCR). Several parameters, particularly the IHC-defined molecular subtypes, are powerful predictors of pCR rate in NACT-treated
BC. However, it is rarely addressed whether BC molecular subtypes change after NACT. Moreover, the influence of post-NACT BC biological categories on patient prognosis has not been explored so far.

Design: In this retrospective study, we compared the tumor histograde (SBR), mitotic index (MI), hormone receptor (HR), HER2 and Ki67 status as well as the IHC-defined molecular subtypes before and after NACT in 282 NACT-treated BC patients (pts) with available pre- and post-treatment tumor samples. We explored the disease-free (DFS) and overall patient survival (OS) among the molecular subtypes after a median follow-up of 10 years.

Results: There were 123 luminal A(Lum A), 67 luminal B (Lum B), 21 HER2+ and 71 triple-negative $(\mathrm{TN})$ tumors. The average pCR rate was $14.5 \%$. Significant variations of HR and HER2 status, MI, Ki67 and SBR grade were observed post-NACT, compared to the pre-NACT values: $22 \%$ of HR-negative tumors switched to positive while $8 \%$ of HR-positive tumors became negative, $\mathrm{p}<10^{-7} ; 63 \%$ of the high Ki67 tumors $(\geq 20 \%$ $\mathrm{Ki} 67+$ cells) switched to the low whereas 10\% of the low Ki67 tumors became Ki67high after NACT, $\mathrm{p}<10^{-7} ; 70 \%$ of high MI tumors (MI 2-3) became low (MI 1) and 11\% of the low MI became high, $\mathrm{p}<10^{-7}$; finally, $11 \%$ of SBR grade 1 tumors became SBR 2 or 3 and $18 \%$ of the SBR 2 or 3 became SBR $1, p<10^{-7}$. About $8 \%$ of BC initially classified as Lum A became Lum B and $29 \%$ of the Lum B switched to Lum A. $35 \%$ of BC initially TN became non-TN and $5 \%$ of the initially non-TN became TN after NACT. Lum A BC that switched to Lum B had a shorter survival than the stable Lum $A(p=0.0095)$. Pre-NACT TN which switched to non-TN had a better prognosis than the stable TN $(p=0.025)$. In pts which reached a pCR, no difference in OS and DFS was found among the molecular subtypes. However, among the pts with a residual disease, the survival of the non-TNBC was significantly better compared to the TNBC $\left(\mathrm{p}=2.6 \times 10^{-5}\right)$.

Conclusions: This study confirms that important changes in the IHC-defined BC molecular subtypes can occur after NACT and influence patient outcome. They should be taken into account in patient management.

292 Adverse Prognostic Significance of BRG1 Protein Expression in Invasive Mammary Carcinoma

K Pinheiro, O Voronel, G Niu, BVS Kallakury, GM Sheehan, CE Sheehan, AB Boguniewicz, JS Ross. Albany Medical College, Albany, NY; Georgetown University Hospital, Washington, DC.

Background: BRG1 protein is encoded by the SMARCA4 gene and serves as a core component of the mammalian chromatin remodeling complex that regulates gene expression. BRG1 has been implicated in the development of a variety of human cancers including breast carcinoma, but the prognostic significance of BRG1 expression determined in clinical breast cancer samples has not been fully elucidated.

Design: Formalin-fixed, paraffin-embedded tissue sections from 125 cases of invasive mammary carcinoma [99 ductal carcinomas (IDC) and 26 lobular carcinomas (ILC)] were immunostained by a manual method (DAKO LSAB+ System-HRP) using rabbit monoclonal BRG1 (Abcam, Cambridge, MA) antibody. Cytoplasmic and/or nuclear immunoreactivity was scored based on intensity and percentage of positive cells in both the tumor and adjacent benign epithelium (when present) in each case. Results were correlated with morphologic and prognostic variables.

Results: Tumor immunoreactivity was predominantly nuclear and intense diffuse nuclear BRG1 overexpression was observed in 83/125 (66\%) tumors. This correlated with high tumor grade $(\mathrm{p}=0.01)$ and advanced tumor stage $(\mathrm{p}=0.023)$ overall; and within the IDC subgroup, correlated with high tumor grade $(\mathrm{p}=0.005)$, advanced tumor stage $(\mathrm{p}=0.012)$ and HER+ status $(\mathrm{p}=0.05)$. Within the ER+ subgroup, intense diffuse nuclear immunoreactivity correlated with high tumor grade $(\mathrm{p}=0.003)$ and HER + status $(\mathrm{p}=0.021)$ with a trend toward PR- status $(\mathrm{p}=0.067)$. While variable BRG1 immunoreactivity was noted in the adjacent benign epithelium, intense diffuse overexpression was not noted; and further, the myoepithelial component of the benign elements was negative. BRG1 overexpression did not correlate with disease-free or overall survival.

Conclusions: BRG1 overexpression is associated with adverse prognostic factors including high tumor grade, advanced pathologic stage and HER2 positive status. Further study of BRG1 expression in mammary carcinoma appears warranted.

\section{Papillary Carcinomas of the Breast Have Distinctive Transcriptomic} Profiles and Are Not Underpinned by a Highly Recurrent Fusion Gene

$S$ Piscuoglio, CK Ng, LG Martelotto, C Eberle, CF Cowell, R Natrajan, F-C Bidard, PM Wilkerson, O Mariani, A Vincent-Salomon, B Weigelt, JS Reis-Filho. Memorial Sloan-Kettering Cancer Center, New York, NY; Institute of Cancer Research, London, United Kingdom; Institut Curie, Paris, France.

Background: Papillary carcinomas (PCs) are a rare special histological type of breast cancer, classified into three histological subtypes, namely encapsulated (EPC), solid (SPC) and invasive (IPC) PCs. We sought to determine whether PCs would constitute a molecular entity distinct from grade- and estrogen-receptor (ER)-matched invasive ductal carcinomas of no special type (IDC-NST) at the transcriptomic level, and whether the histological subtypes of PCs would have distinct transcriptomic profiles and patterns of gene copy number aberrations. Finally, we investigated whether PCs would be driven by a highly recurrent fusion gene or driver mutation.

Design: RNA and DNA were extracted from PCs (4 SPCs, 5 IPCs and 7 EPCs) and grade/ER-matched IDC-NSTs (RNA only). RNA samples from 16 PCs and 16 grade/ ER-matched IDC-NSTs were subjected to microarray-based gene expression profiling (Illumina HT-12 v4). Intrinsic subtypes were defined using a microarray version of PAM50. Copy number profiling was performed using Affymetrix SNP6.0 arrays. Eight PCs (3 IPCs, 3 EPCs, 2 SPCs) were subjected to paired-end RNA sequencing (Illumina GAIIx); expressed fusion transcripts were identified using deFuse and Chimerascan, and confirmed by RT-PCR. 
Results: PCs were preferentially of grade I/II ( $82 \%$ ) and ER-positive ( $100 \%)$. PAM50 subtyping revealed that 10,5 and 1 cases were classified as of luminal A, luminal B and basal-like subtypes, respectively. Unsupervised hierarchical cluster analysis revealed that PCs preferentially clustered together, separately from IDC-NSTs. Supervised analysis demonstrated that PCs displayed lower expression levels of cellular growth and proliferation-related genes than IDC-NSTs. Although PC subtypes displayed similar patterns of copy number aberrations, significant differences in the transcriptomic profiles of EPCs, SPCs and IPCs were found, including overexpression of RET in SPCs. Although 15 in-frame fusion genes were identified and validated, none was shown to be recurrent. Mutations in PIK3CA, TP53, MAP $3 K 1, A K T 1$ and MAMDC4 were found, but mainly in single samples.

Conclusions: PCs are preferentially of luminal subtype. At the transcriptomic level, PCs are distinct from grade/ER-matched IDC-NSTs. Our findings further demonstrate that PCs are not underpinned by a highly recurrent expressed fusion gene or point mutation.

\section{GATA3 Is a Marker of Estrogen Receptor-Positive but Not Estrogen Receptor-Negative Breast Cancers}

$S$ Piscuoglio, YH Wen, LG Martelotto, E Guerini Rocco, M Akram, AA Jungbluth, E Brogi, B Weigelt, JS Reis-Filho. Memorial Sloan-Kettering Cancer Center, New York, NY.

Background: GATA3 mRNA levels have been shown to be differentially expressed between estrogen receptor (ER)-positive (ER+) and ER-negative (ER-) breast cancers, and to correlate strongly with the expression levels of ESR1 (the gene encoding ER). Recent immunohistochemical (IHC) analyses, however, have claimed that $43 \%$ of triplenegative breast cancers express GATA3. Based on these results, it has been suggested that GATA3 could be employed as a marker to differentiate breast cancer from other epithelial malignancies. Here we sought to define the levels of GATA3 mRNA and protein expression in ER+ and ER- breast cancer cell lines and primary breast cancers. Design: GATA3 mRNA and protein expression levels were analyzed in $9 \mathrm{ER}+$ and 7 ER- breast cancer cell lines using real-time PCR (qRT-PCR) and western blotting, respectively. Sections of formalin-fixed paraffin embedded (FFPE) cell line pellets were subjected to IHC analysis using a commercial anti-GATA3 monoclonal antibody (clone L50-823, Biocare). Using the same IHC methods, GATA3 expression was analyzed in a tissue microarray containing $129 \mathrm{ER}+/ \mathrm{HER} 2-, 23 \mathrm{HER} 2+$ and 211 triple-negative breast cancers. Representative FFPE full-face sections of breast cancers defined as $\mathrm{ER}+/ \mathrm{HER} 2-/ \mathrm{GATA} 3+(\mathrm{n}=5)$, triple-negative/GATA3 $+(\mathrm{n}=5)$ and triple-negative/ GATA3- $(\mathrm{n}=5)$ by IHC analysis were microdissected and subjected to RNA extraction and GATA3 qRT-PCR analysis.

Results: All ER+ breast cancer cell lines expressed GATA3 both at the mRNA and protein levels as defined by qRT-PCR and western blotting respectively, whereas ERbreast cancer cell lines lacked expression of GATA3. IHC analysis of the FFPE cell line pellets confirmed the expression of GATA3 in ER+ cell lines; surprisingly, however, ER- cell line pellets also displayed strong GATA3 immunoreactivity. RT-PCR analysis of immunohistochemically ER+/HER2-/GATA3 + breast cancers revealed significantly higher GATA3 mRNA expression levels than those detected in triple-negative/GATA3+ and triple-negative/GATA3- $(\mathrm{p}=0.008)$. No difference in the mRNA expression levels of GATA3 was detected between triple-negative breast cancers that were GATA3+ or GATA3- by IHC analysis.

Conclusions: GATA3 expression is preferentially found in ER+ breast cancers, and not detected in the vast majority of ER- lesions. The antibody clone L50-823 seems to cross-react with proteins other than GATA3 in IHC assays performed on FFPE tissue samples. Our results do not support the contention that GATA3 IHC analysis can be employed to differentiate breast cancers from other epithelial malignancies reliably.

\section{Scoring HER2 Dual In Situ Hybridisation in Breast Cancer Borderline Categories: How Many Cells Is Enough?}

$S$ Prendeville, L Feeley, MW Bennett, F O'Connell, TJ Browne. Cork University Hospital, Cork, Ireland.

Background: Breast cancer cases with borderline HER2 Dual Color In Situ Hybridization DNA (Dual ISH) results pose both diagnostic and treatment dilemmas. The appropriate number of cells to score in borderline cases is controversial with the majority of published guidelines relating to HER2 fluorescence in situ hybridization (FISH). Recent publications suggest that scoring additional cells in borderline cases may be advantageous. This study evaluates whether scoring $>40$ cells in borderline cases using Dual ISH method yields different ratios, altering HER2 status and treatment. Design: All invasive breast carcinomas with equivocal (2+) HER 2 immunohistochemistry (IHC) and borderline Dual ISH (Ventana INFORM Assay) results scored by 2 pathologists over 1 year were identified at our insititution. HER2 IHC (Ventana, 4B5) was evaluated in each case to identify the areas of strongest membrane staining. These areas were selected for Dual ISH scoring and 20 cells as per manufacturer's guidelines scored. A subsequent 20, 40, 60, 80 and 100 cells were scored with individual HER2/ Chr17 ratios, mean HER2 and Chr17 copy numbers calculated for each set of 20 cells. Cases were classified into HER2 negative, positive, borderline negative (Her2:Chr17 1.8-1.99) or borderline positive (Her2:Chr17 2.0-2.20).

Results: 16 cases with a Dual ISH borderline result were identified: 13 borderline negative ( $82 \%)$ and 3 borderline positive (18\%). In 13 cases $(82 \%)$ the HER $2 /$ Chr17 ratio remained in the borderline category regardless of the number of cells counted. In 2 cases, the result changed from borderline positive to borderline negative after counting 120 cells and in 1 case the HER 2 result became negative after counting 100 cells.

\begin{tabular}{|c|c|c|c|c|c|}
\hline \begin{tabular}{|l|} 
Case No. \\
\end{tabular} & 40 cells & 60 cells & 80 cells & 100 cells & 120 cells \\
\hline 1 & 1.98 & 1.90 & 1.90 & 1.89 & 1.87 \\
\hline 2 & 1.90 & 1.91 & 1.90 & 1.85 & 1.83 \\
\hline 3 & 1.81 & 1.82 & 1.82 & $1.78^{*}$ & $1.75^{*}$ \\
\hline 4 & 2.06 & 2.06 & 2.01 & 2.0 & $1.96^{*}$ \\
\hline 5 & 1.95 & 1.88 & 1.90 & 1.88 & 1.89 \\
\hline 6 & 1.89 & 1.86 & 1.84 & 1.82 & 1.82 \\
\hline 7 & 1.98 & 1.99 & 1.91 & 1.88 & 1.87 \\
\hline 8 & 1.97 & 1.94 & 1.95 & 1.94 & 1.91 \\
\hline 9 & 1.91 & 1.93 & 1.91 & 1.89 & 1.91 \\
\hline 10 & 1.91 & 1.89 & 1.93 & 1.86 & 1.83 \\
\hline 11 & 2.18 & 2.09 & 2.11 & 2.08 & 2.05 \\
\hline 12 & 1.91 & 1.94 & 1.91 & 1.91 & 1.85 \\
\hline 13 & 2.18 & 2.11 & 2.05 & 2.03 & $1.96^{*}$ \\
\hline 14 & 1.83 & 1.82 & 1.83 & 1.81 & 1.80 \\
\hline 15 & 1.87 & 1.82 & 1.85 & 1.82 & 1.82 \\
\hline 16 & 1.82 & 1.87 & 1.88 & 1.83 & 1.80 \\
\hline
\end{tabular}

Conclusions: Utilizing Her2 IHC identifies the appropriate area for Dual ISH scoring. Counting of additional cells (up to 80 ) after scoring 40 cells does not change the overall HER2 category. Interestingly when $>100$ cells are counted the HER2/Chr17 ratio decreases and in 2 cases altered the HER2 category with treatment implications. This change is likely due to moving out of the area of strongest signal.

296 Optimal Scoring of Dual Color In Situ Hybridization (Dual ISH) for Evaluation of HER2 Amplification in Breast Carcinoma: Careful Immunohistochemical Assessment Is Equivocal to Scoring Additional Fields and Cells

SPrendeville, CFives, L Feeley, MW Bennett, TJ Browne, F O'Connell. Cork University Hospital, Cork, Ireland.

Background: Dual color in situ hybridization DNA Assay (Dual ISH) HER2 testing in breast carcinoma is increasing in clinical use. Scoring guidelines for HER2 fluorescence in situ hybridization (FISH) are well established. Data regarding optimal scoring of HER2 Dual ISH, particularly in heterogeneous cases, is more limited with recent publications recommending that scoring of additional cells and/or fields may be advantageous. This study evaluates, whether in conjunction with HER2 immunohistochemistry (IHC), scoring more than 20 cells and additional fields (up to 3 fields) is of benefit in HER2 evaluation using a Dual ISH method.

Design: 100 consecutive breast cancer cases with equivocal HER 2 immunohistochemistry (IHC) results (Ventana 4B5, CAP Guidelines Score 2+) and corresponding Dual ISH (Ventana INFORM Assay) were retrieved from institutional files for a six month period. Dual ISH results in the borderline range (HER2:Chr17 ratio 1.8 to 2.2) were excluded. All cases were dual reported by two breast pathologists. In each case HER2 IHC was reviewed to identify the area of strongest membrane reactivity. This corresponding area was targeted for Dual ISH scoring. Scoring was performed according to manufacturer's guidelines for 20 cells. Additional cells were scored in a similar fashion and cumulative counts and HER2:Chr1 7 ratios calculated for 40 and 60 cells. Ratios were also generated for 2 additional fields within the targeted area. All cases were categorised as positive/ amplified (HER2:Chr17 ratio $>2.2$ ) or negative/non-amplified (HER2:Chr17 ratio $<1.8$ ). Results: 11 cases were classified as HER2 positive/amplified and 89 cases as HER2 negative/non-amplified following an initial count of 1 field and 20 cells. HER2 status as determined by HER $2 / \mathrm{Chr} 17$ ratio or absolute HER2 copy number was unchanged between the 3 fields ( $100 \%$ concordance). HER 2 status as determined by HER $2 / \mathrm{Chr} 17$ ratio or absolute HER2 copy number was unchanged following scoring of 40 and 60 cells $(100 \%$ concordance). Statistical analysis showed with $95 \%$ confidence that for HER2:Chr17 ratios between 1.5 and 2.5 there was a true concordance rate in excess of $99.7 \%$.

Conclusions: HER2 IHC is essential to identify appropriate target areas for Dual ISH scoring, particularly in heterogeneous cases. Using this approach for non borderline cases, scoring of additional fields (up to 3 ) and additional cells (up to 60) did not alter the results obtained after scoring 1 field and 20 cells.

297 The Incidence and Histologic Type of Carcinoma in Patients First Presenting with Axillary Lymph Node Metastases

E Racila, T Khoury. Roswell Park Cancer Institute, Buffalo, NY.

Background: Metastases to the axillary lymph nodes are often encountered in the clinical assessment of patients with cancer, most frequently caused by melanoma or breast cancer. However, when patient's first presentation is axillary lymph node metastasis, the determination of the origin solely on pathologic ground can be challenging. We sought to determine the incidence and the gender-related differences between the histologic types of non-melanocytic epithelial tumors that first present as metastatic disease to the axilla.

Design: We reviewed the pathology reports of cases with metastases to axillary lymph nodes in our database. We identified 122 patients, 96 women and 26 men, with metastatic carcinoma to the axillary lymph nodes as the first clinical sign of presentation. The information analyzed included the age and gender, the histologic features of the lesion and phenotype of tumor resulting from immunohistochemistry, the patient's history of cancer and the date of primary tumor identification if it later became available.

Results: Table 1 illustrates the histologic types and site of origin with relation to gender. The average age at diagnosis among all patients was 62 years with no significant gender-related difference. Histologic examination and ancillary studies were unable to identify the origin in $46(48 \%)$ cases. In women, 48 of 96 tumors (50\%) turned out to be of mammary phenotype, sixteen (33\%) of which were occult. Poorly differentiated carcinoma of unknown origin was more common in women than men, $38 \%$ vs. $12 \%$, respectively $(\mathrm{p}=0.029)$. 
Conclusions: In the setting of first presentation of cancer in axillary lymph node, classifying the tumor can be challenging. We found gender related differences of various histologic types of metastatic carcinoma to axillary lymph nodes. When a tumor has mammary carcinoma phenotype, the incidence of occult breast carcinoma is high, accounting for about one third of the cases.

\begin{tabular}{|c|c|c|c|}
\hline 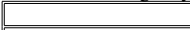 & Women(\%) & $\operatorname{Men}(\%)$ & Fisher Test \\
\hline Breast Primary & $48(50)^{*}$ & 0 & \\
\hline Non-Breast Cases & $48(100)$ & $26(100)$ & \\
\hline Adenocarcinoma & $15(31)$ & $4(15)$ & 0.17 \\
\hline Lung & 12 & 1 & \\
\hline GI Tract & 1 & 2 & \\
\hline Ovarian & 1 & & \\
\hline Unknown & 1 & 1 & \\
\hline Squamous Cell & $7(15)$ & $8(31)$ & 0.13 \\
\hline Skin & 1 & 0 & \\
\hline Unknown & 6 & 8 & \\
\hline Neuroendocrine & $5(10)$ & $7(27)$ & 0.097 \\
\hline Small Cell-Lung & 1 & 3 & \\
\hline Small Cell-Unknown & 2 & 1 & \\
\hline Large Cell & 2 & 3 & \\
\hline Merkel Cell & $3(6)$ & $3(12)$ & \\
\hline Papillary & 0 & 1 & \\
\hline Poorly Differentiated & $18(38)$ & $3(12)$ & 0.029 \\
\hline Total & 96 & 26 & \\
\hline
\end{tabular}

*Percent of total number of female patient

298 EGFR, HER3 and MET Immunohistochemistry Can Help Tailoring Targeted Therapy of Triple-Negative Breast Cancer

$N$ Radosevic-Robin, C Abrial, M-M Dauplat, J-M Nabholtz, F Penault-Llorca. Jean Perrin Cancer Center and ERTICa Research Group, University of Auvergne, ClermontFerrand, France.

Background: The standard neoadjuvant chemotherapy (NACT) of triple-negative breast cancer (TNBC) leaves around $80 \%$ of patients (pts) with a residual tumor (RT). Drugs targeting membrane receptors could improve the efficacy of NACT in TNBC, however, simple tools able to guide that therapy type are lacking. We investigated whether immunohistochemical (IHC) analysis of EGFR, HER3 and MET expression can help creating a more specific treatment for TNBC.

Design: The study included pts with a post-NACT RT in 2 pilot clinical trials of anti-EGFR antibodies in TNBC, conducted from 2009 to 2011 by our team. One trial combined panitumumab with standard fluorouracil-epirubicin-cyclophosphamide and docetaxel (P-FEC-T) while another applied cetuximab with docetaxel (C-T). EGFR, HER3 and MET were assessed by IHC on pre- and post-NACT BC tissue. Expressions were evaluated by Quick score. At least 30 points of difference qualified the post-NACT score as changed, compared to the baseline.

Results: 44 pts were eligible (P-FEC-T: 26 pts, C-T: 18 pts). Using 150 as cut-off we separated the pts in each study on pre-NACT EGFR high (score $\geq 150$ ) and EGFR low (score $<150$ ). The EGFR high BC in P-FEC-T study most frequently showed EGFR decrease (9/13), HER3 increase (4/6) and MET decrease (7/12) after NACT. However, the EGFR low tumors mostly had no change in EGFR (7/13) and HER3 (5/9) but increased MET (7/11) (Table 1).

\begin{tabular}{|c|c|c|c|c|c|c|}
\hline \multicolumn{7}{|c|}{ PRE-NACT EGFR SCORE } \\
\hline & \multicolumn{3}{|c|}{$\mathrm{HIGH}$} & \multicolumn{3}{|c|}{ LOW } \\
\hline median & \multicolumn{3}{|c|}{220} & \multicolumn{3}{|c|}{0} \\
\hline $\mathrm{n}$ & \multicolumn{3}{|c|}{13} & \multicolumn{3}{|c|}{13} \\
\hline \multicolumn{7}{|c|}{ POST-NACT COMPARED TO PRE-NACT } \\
\hline & down & flat & up & down & flat & up \\
\hline EGFR & 9 & 3 & 1 & 2 & 7 & 4 \\
\hline HER3 & 0 & 2 & 4 & 0 & 5 & 4 \\
\hline MET & 7 & 4 & 1 & 1 & 3 & 7 \\
\hline
\end{tabular}

In C-T study, the EGFR high BC showed a similar pattern of score change post-NACT as in P-FEC-T: frequent EGFR decrease (4/7), HER3 increase (5/7) and MET decrease (4/7). The EGFR low BC were more heterogeneous post-NACT than in P-FEC-T: EGFR was mostly increased (4/13) or unchanged (4/13), HER3 increased (5/13) as well as MET (5/13). Of note, the C-T EGFR low group had a higher median EGFR score than the same group in P-FEC-T (Table 2).

PRE-NACT EGFR SCORE

\begin{tabular}{c|c|c|c|c|c|c}
\hline & \multicolumn{3}{|c|}{ HIGH } & \multicolumn{3}{c}{ LOW } \\
\hline median & \multicolumn{3}{|c|}{210} & \multicolumn{3}{c}{70} \\
\hline $\mathrm{n}$ & \multicolumn{3}{c|}{71} \\
\hline \multicolumn{6}{|c|}{ POST-NACT COMPARED TO PRE-NACT } \\
\hline & down & flat & up & down & flat & up \\
\hline EGFR & 4 & 2 & 1 & 3 & 4 & 4 \\
\hline HER3 & 0 & 2 & 5 & 2 & 4 & 5 \\
\hline MET & 4 & 3 & 0 & 3 & 3 & 5 \\
\hline
\end{tabular}

Conclusions: We conclude that pre-therapy EGFR high TNBC will likely downregulate EGFR and upregulate HER3 as a resistance mechanism to EGFR-targeted NACT, while the pre-therapy EFGR low TNBC will react mainly by upregulating HER3 and MET. Further development of an IHC-based signature, able to guide introduction of EGFR-, HER3- and MET-targeting agents in TNBC therapy, is ongoing in our group.

299 Is Routine Surgical Excision Necessary for Radial Scar on Core Biopsy?: A Large Retrospective Study in an Academic Women's Center AC Ranade, DK Samrao, C Zhao. Magee Womens Hospital of UPMC, Pittsburgh, PA. Background: The clinical management of radial scar detected on needle core biopsy (NCB) remains controversial. There is a great discrepancy of reported up-grade rate at excision, ranging from $0 \%$ to over $30 \%$; the most important reason being, the small case numbers and different criteria for follow-up surgery.

Design: A computer based search was carried out on our database from July 2005 to January 2011, to identify the cases of radial scar on NCB. The pathological findings in follow up excision (FUE) and time between biopsy and FUE was recorded and analyzed. Results: 382 cases were interpreted as radial scar (RS). 168 cases were excluded due to prior history of or synchronous malignant breast lesions or ADH/ALH. Of the 214 cases of RS, $124(57.9 \%)$ had FUE. The mean interval between NCB and FUE was 1.9 months (range 1-11 months). The discovery of significant lesions upon surgical excision of RS is shown in (table 1). DCIS and invasive carcinoma was found in 2 cases $(1.6 \%)$ on FUE.

Conclusions: In the current study we found a cancer upgrade rate of $1.6 \%$, while upgrade to atypia and lobular neoplasia was $24.8 \%$ and $4.8 \%$ respectively. Therefore we conclude that, RS associated with benign findings on a CNB need not be excised owing to the low rate of associated carcinoma and hence, routine imaging follow-up may be performed.

Findings on subsequent breast excision (124 cases)

\begin{tabular}{|l|l|l||}
\hline UPGRADE LESION ON EXCISION & Number of cases & Percentage (\%) \\
\hline DCIS & 1 \\
\hline IDC & 1 & 0.8 \\
\hline ALH/LCIS & 6 & 0.8 \\
\hline ADH & 28 & 4.8 \\
\hline FEA & 3 & 22.4 \\
\hline
\end{tabular}

300 Factors Affecting the Cold Ischemic Time of Breast Resection Specimen: A Single Institution's Experience

$B$ Ren, L Baxter, ML Fuller, RE Sage, NLoria, P Tang, K Skinner, DG Hicks. University of Rochester Medical Center, Rochester, NY.

Background: Cold ischemic time (CIT) is a significant pre-analytic factor that may negatively impact the measurement of protein antigenicity for prognostic factor testing in breast cancer. The American Society of Clinical Oncology/College of American Pathologists guidelines suggest the CIT for breast cancer specimens to be less than 1 hour, which is a challenge for handling breast lumpectomy and mastectomy specimens in the routine clinical setting. We have performed a retrospective review of cases in our institute and tried to identify the major factors affecting CIT of breast resection specimens.

Design: Ninety-three breast resection specimens collected in the first 6 months of 2013 in our institute were reviewed. 94\% (87/93) of specimens had complete documentation of removal time, receiving time, and time-to formalin fixation (CIT), and they were classified into two groups based on the CIT ( $>60 \mathrm{~min}$. and $\leq 60 \mathrm{~min}$.).

Results: Among the 87 cases with complete documentation, $61 \%(53 / 87)$ had a CIT $\leq 60$ min. (mean=43 min.), including 28 lumpectomies and 25 mastectomies. 39\% (34/87) had a CIT $>60 \mathrm{~min}$. (mean=78 min.), including 27 lumpectomies and 7 mastectomies. In $>60$ min. group, the means of both removal to receiving time, and receiving to fixation time are significantly greater than those in $\leq 60 \mathrm{~min}$. (32 and $49 \mathrm{~min}$. vs. 14 and $29 \mathrm{~min}$., $\mathrm{p}<0.001)$. The lumpectomy specimens in $>60 \mathrm{~min}$. group had a larger specimen size and a greater number of new margins removed in the same procedure compared with those in $\leq 60 \mathrm{~min}$. group (average $5.3 \mathrm{~cm}$ and 0.86 new margins vs average $7.2 \mathrm{~cm}$ and 2.98 new margins, $\mathrm{P}<0.001$ ). The CIT of specimens submitted by 2 surgeons (A, 76 cases and B, 20 cases) also were compared. The means of removal to receiving time and CIT of specimens submitted by surgeon A are significantly less than surgeon B (15 and $52 \mathrm{~min}$. vs 35 and $79 \mathrm{~min}$.). 47\% (16/34) of specimens from $>60 \mathrm{~min}$. group were received around noon (between 11:30 am and 1:30 pm) compared to $21 \%(11 / 53)$ of specimens from $\leq 60 \mathrm{~min}$. group, which may be associated with a staff shortage during the lunch time.

Conclusions: Minimizing CIT was shown to be important for accurate prognostic factor testing in breast cancer. Our data suggests the CIT of breast resection specimens is affected by multiple factors including specimen type, complexity, receiving time and surgeons. Close collaboration and joined efforts among surgeons, pathologists and support staff are necessary for standardization of specimen handling and improvement of CIT.

301 IgG4-Related Sclerosing Mastitis (IgG4-RSM) of the Breast: An Uncommon Entity and Diagnostic Challenge on Core Biopsy (CB)

E Resetkova, A Padula. MDACC, Houston, TX; Genoptix Medical Laboratory, Carlsbad, CA.

Background: IgG4-related sclerosing disease, is an uncommon emerging entity, with clinical and radiologic features mimicking malignancy in multiple organs. It is characterized by a diffuse and mass-forming inflammatory reaction with an infiltrate rich in IgG4-positive plasma cells associated with fibrosclerosis and obliterative phlebitis. Breast involvement has been rarely reported, and due to its rarity, diagnostic criteria 
are not well-defined. Diagnosis on CB may be limited due to sample size. We report on clinical presentation and offer guide to pathologic work-up of IgG4-RSM on CB samples in six patients with chronic inflammatory infiltrates in breast.

Design: Six female patients (age, 23 to 73 years) presented with palpable and painful masses. On imaging, patients had features suspicious for malignancy (BI-RADS 4 and 5). On MRI, two patients showed enhancing high-density spiculated masses suspicious for malignancy. IgG (DAKO, 1:40,000) and IgG4 (Light Technologies, Ltd., 1:150) were performed. IgG4 positivity ( $>50$ cells/HPF) and increased ratio of IgG4/IgG-positive cells $(>40 \%)$ was considered suggestive of IgG4-RSM. Serum IgG/IgG4 levels were measured in 2 cases and were negative.

Results: Microscopic studies revealed chronic granulomatous mastitis in 3 cases, composed of lymphoplasmacytic infiltrate, multinucleated giant cells, non-caseating granulomas, loss of acinar architecture, and fibrosclerosis. In one case inflammatory pseudotumor was suggested. Two patients had lymphohistiocytic infiltrate. Acid fast bacilli, and GMS stains were negative in all cases. Two patients underwent work up for lymphoma due to associated axillary lymphadenopathy that was negative. There were increased number of IgG4-positive polyclonal plasma cells in 3 cases of chronic granulomatous mastitis with increased ratio of $\mathrm{IgG} / \mathrm{IgG} 4$ seen in 2 cases. No obliterative phlebitis was noted in any case. In remaining cases, only minimal IgG4 positivity was seen

Conclusions: Histopathologic study is essential to determine non-malignant nature of chronic inflammatory infiltrate and rule out infectious conditions associated with granulomatous reactions. In our cohort, increased IgG4-positive plasma cells were identified in 3 cases with chronic granulomatous process. Although the etiology of IgG4 RSM is currently unknown, the excellent outcome obtained with steroid treatment in other organs is very suggestive of an autoimmune basis and it may offer possible new therapy in selected cases with increased number of IgG4 positive cells.

302 Pathologic-Radiologic Correlation in Evaluation of Retroareolar Margin in Nipple Sparing Mastectomy

MP Riley, AL Chetlen, S Schetter, J Mack, CS Hollenbeak, B Han, DM Karamchandani. Penn State Milton S. Hershey Medical Center, Hershey, PA.

Background: Nipple-sparing mastectomy (NSM) has recently been increasing in popularity due to a better cosmetic outcome and quality-of-life benefit. Proper patient selection is imperative for disease free margins and preservation of the nipple-areola complex (NAC). Radiologic distance between the tumor and the nipple is independently predictive of NAC involvement and can assist in patient selection for NSM. However, in these situations, concordance between imaging and histologic evaluation would play a major role in making patient selection for NSM meaningful. Hence, the present study analyzes the pathologic-radiologic correlation (PRC) to evaluate retroareolar (RA) margin in patients undergoing NSM

Design: A retrospective histologic and radiologic review of 80 NSM performed on 45 patients between 2010-2013 was done. Histologically, the cases were divided into positive (defined as invasive or in situ carcinoma within $5 \mathrm{~mm}$ of the RA margin) and negative (greater than $5 \mathrm{~mm}$ from the RA margin). Three breast radiologists independently and blindly reviewed mammographic and MRI images from these patients. Radiographically, positive cases were defined as suspicious enhancement and/or suspicious findings within $20 \mathrm{~mm}$ of the nipple on MRI and/or diagnostic mammography respectively. A kappa coefficient was estimated to measure agreement between histologic and radiologic findings.

Results: 69 of $80(86.25 \%)$ cases had negative RA margins based on both histologic and radiologic review. Six cases were discordant (7.5\%). Agreement between pathology and radiology was moderate and statistically significant as indicated by a kappa coefficient of $0.59(\mathrm{p}<0.0001)$

\begin{tabular}{|c|c|c|c|}
\hline Pt No. & $\begin{array}{l}\text { Distance from nipple in } \\
\text { mm on imaging }\end{array}$ & Histologic evaluation & Results \\
\hline 11 & 3 & Neg* & $\mathrm{D}$ \\
\hline 13 & 10 & Neg* & D \\
\hline 15 & 18 & Neg* & D \\
\hline 17 & 14 & $4 \mathrm{~mm}$ IDC \& DCIS & $\mathrm{C}$ \\
\hline 18 & 13 & Neg* & D \\
\hline 19 & 17 & $1 \mathrm{~mm}$ IDC; $3 \mathrm{~mm}$ DCIS & $\mathrm{C}$ \\
\hline 20 & 12 & $0 \mathrm{~mm}$ LCIS & $\mathrm{C}$ \\
\hline 22 & 12 & $0.5 \mathrm{~mm}$ DCIS & $\mathrm{C}$ \\
\hline 41 & 0 & $0 \mathrm{~mm}$ IDC, DCIS & $\mathrm{C}$ \\
\hline
\end{tabular}

* Neg-Distance of $>5 \mathrm{~mm}$ on histology. C-concordant; D-Discordant.

\begin{tabular}{|c|c|c|c|}
\hline Pt No. & Histologic distance from nipple & Imaging & Results \\
\hline 26 & $0.5 \mathrm{~mm} \mathrm{IDC}$ & Neg & $\mathrm{D}$ \\
\hline 40 & $4 \mathrm{~mm}$ DCIS & Neg & $\mathrm{D}$ \\
\hline
\end{tabular}

D-Discordant

Conclusions: There is a significant agreement between histologic and radiologic evaluation for assessment of RA margin, and radiologic imaging results provide valuable information to help select patients for NSM.

303 Multiple Breast Papillomas as a Predictor of Familial Cancer Events W Rizzo, SM Said, MH Frost, TM Allers, LC Hartmann, AC Degnim, DW Visscher. Mayo Clinic, Rochester, MN.

Background: Patients with multiple papillomas may have genetic alterations, such as the PTEN mutation seen in Cowden's disease, that increases the risk of developing a variety of cancers. This study evaluated using the occurrence of multiple papillomas as a surrogate marker for genetic mutation and tracked cancer occurrence in the proband and her family members.
Design: Women with two or more breast papillomas were identified from the Mayo Benign Breast Disease Cohort. This cohort is comprised of 9,376 women who had surgical excisions resulting in benign breast biopsies (1967-1991). All papilloma diagnoses were confirmed by slide reviewed performed by a pathologist with a breast specialization. Proband follow-up and familial information was obtained through study questionnaires.

Results: Multiple breast papillomas were identified in 60 patients between 1967-1991. The mean age of diagnosis was 55 (range 27-83 years). Proband follow-up and familial information was obtained in 50 cases following an average of 15.3 years (range 9-34 years). At the time of follow-up $28 \%$ of patients had developed cancer; $18 \%$ of which were breast cancer. $36 \%$ of the breast cancers were ipsilateral to the prior papillomas. Probands also developed cancer of the ovary(1), thyroid(1), bladder(1) and uterus (1), as well as Hodgkins (1) and non-Hodgkins (1) lymphoma. $76 \%$ of the probands had relatives that developed cancer. The number of affected relatives per proband ranged from 0-12. Amongst the probands that had affected relatives the average number affected was 3.0 family members. $56 \%$ of the probands had first degree relatives that developed cancer. $48 \%$ of probands reported 2 or more relatives that developed cancer. Over 106 relatives developed cancer of which the majority (39\%) was breast cancer. Additional cancer sites included; the small and large intestinal (11.3\%), lung (10.4\%), prostate $(6.6 \%)$, stomach $(5.7 \%)$, pancreas $(3.8 \%)$, ovary $(3.8 \%)$, bone $(3.8 \%)$, bladder $(2.8 \%)$, kidney $(1.9 \%)$, skin $(1.9 \%)$, mouth/throat $(1.9 \%)$, cervix or endometrium $(1.9 \%)$, back and hip (1.9\%), brain $(0.9 \%)$, sinuses $(0.9 \%)$, liver $(0.9 \%)$, and leukemia $(0.9 \%)$. Conclusions: The histologic diagnosis of multiple breast papillomas may be a surrogate marker for a genetic alteration that identifies people at increased risk of developing cancer of varying types, especially breast. Identifying at risk patients can aid in clinical decision making.

\section{Benefits of a Second Opinion in Breast Pathology}

M Rosa, A Magliocco, L Khazai. Moffitt Cancer Center and Research Institute, Tampa, FL.

Background: Examination of outside pathology slides is routinely practiced at our institution before definitive treatment for breast cancer is provided. Previous reports have shown the benefits of a second opinion in breast pathology with an estimated rate of major errors on primary diagnosis ranging between $2-4 \%$.

Design: This prospective study was designed to compare histopathologic diagnoses of cases received for consultation in patients seeking treatment or a second opinion at our institution in a breast pathology subspecialized practice, between March 2013 and July 2013.

Results: A total of 471 cases were included. Discrepancies were divided into major (change course of treatment or prognosis) and minor (did not impact treatment or prognosis). Minor discrepancies included: change of type of malignancy (i.e. lobular vs. ductal), threshold diagnosis (borderline) cases, atypical ductal hyperplasia vs. ductal caricnoma in situ, atypical lobular hyperplasia vs. lobular caricnoma in situ), tumor grade, and location. Major errors included: categorical interpretations (CI), tumor stage, margin status (if re-excision was required), lymph nodes status, and estrogen and progesterone receptors and Her-2 status. Eighty-nine (19\%) discrepancies were identified. Nineteen cases constituted major discrepancies (4\%) and 70 cases constituted minor discrepancies (15\%). Within the major discrepancies, margin status was the most prevalent ( $42 \%)$, followed by categorical interpretation and receptor status ( $16 \%$ each). Three cases of CI included change from benign to malignant (missed invasive tumor), malignant to benign (microglandular adenosis clasiffied as invasive carcinoma by the primary pathologist) and DCIS to invasive carcinoma. These three changes significantly affected treatment course. Within the minor discrepancies, the most common changes were tumor grade (21\%), change on type of malignancy (20\%), and difference of opinion in borderline cases on core needle biopsy (14\%).

Conclusions: Our data confirm the value of a second opinion on breast pathology to improve medical treatment and guide surgery planning. Categorical changes in diagnosis and evaluation of margin and prognostic/ predictive factors status represented the majority of major discrepancies that impacted patient care. More in-depth training as well as practice and reporting standardization in breast pathology are necessary to improve patient care.

\section{Exome Sequencing of Mammospheres and Primary Tumors} Indicates Dynamic Transitions between Stem-Like and Differentiated States in Breast Cancer

G Rosin, D Klevebring, R Ma, I Fredriksson, J Bergh, J Hartman. Karolinska Institutet, Stockholm, Sweden; Karolinska University Hospital, Stockholm, Sweden.

Background: Breast cancer cells with phenotypical similarities to adult mammary stem cells have been shown to initiate and maintain tumor growth and therefore named cancer stem cells (CSC). There is substantial overlap of phenotypic markers with the biological capacity to form non-adherent cell spheres in vitro, named mammospheres. Based on the CSC concept, two alternative and opposing explanations have been proposed to describe the tumorigenic process governed by CSC; (1) the CSCs are an early-tumor initiating clone with capacity of asymmetric cell-divisions and subsequent differentiation to yield intratumoral heterogeneity; (2) the CSC phenotype is dynamic and reversible and can be adopted by any cancer epithelial cell through epigenetic de-differentiation. Design: To understand which of these models is the predominant, governing the development of breast cancer, we collected paired bulk tumor samples, in vitro purified mammospheres and normal leukocyte DNA from ten patients. The sequencing was run with Illumina Hiseq 2000 using an exome capture kit.

Results: Across all patients, $80 \%$ of the mutations are on average shared between the tumor and CSC-fraction (range 45\%-91\%). We hypothesize that if the two cell populations, differentiated cells and CSC, were strictly separated, the number of mutations in the CSC would be very low due to low replication rate of that cell type 
compared to the continuously proliferating epithelial cells. In such a scenario, very few mutations would also be shared between CSC and bulk tumor cells. In contrast, we see a large degree of shared mutations. A cell-state model where inter-conversion between cell types occurs, would explain the large degree of shared mutations. As mutations occur in the rapidly dividing epithelial cells, they are propagated to daughter cells that are occasionally converted into CSCs.

Conclusions: Our data points towards the support of a model where the epithelial cells of the bulk tumorin vivo are able to phenotypically revert into CSC with stem cell properties and vise versa (model 2). This implicates that any of the epithelial cells of bulk tumor may acquire stem cell properties, making a stem cell targeted treatment approach increasingly complex.

306 A Comparison of Estrogen Receptor, Progesterone Receptor and HER2 Profiles Pre and Post Neoadjuvant Treatment in Breast Carcinoma Cases

C Ryan, L Feeley, MW Bennett, TJ Browne, F O'Connell. Cork University Hospital, Cork, Ireland.

Background: Neoadjuvant chemotherapy is increasingly in use for treatment of breast carcinoma. The Estrogen Receptor (ER). Progesterone Receptor (PgR) and HER2 profile of a primary breast carcinoma is essential in determining treatment. Data regarding the effects of neoadjuvant therapy on biomarker profile (ER, PgR and HER2 status) is limited and conflicting. Post-treatment hormone receptor and HER2 status may be clinically relevant in guiding further management. The aim of this study was to compare the ER, PgR and HER2 profile of breast tumors pre and post neoadjuvant treatment. Design: Neoadjuvant treated breast carcinoma cases were identified from institutional files over a 1 year period. ER, PgR and HER2 results were compared pre and post treatment for all cases. Immunohistochemistry (IHC) for hormone and HER2 status was reported according to CAP guidelines. Cases which yielded an equivocal HER2 result by IHC ( $2+$ score) were evaluated using a Dual color DNA in situ hybridization assay (dual ISH). IHC and dual ISH assays were performed using validated methods in a single laboratory. IHC and Dual ISH tests were evaluated by 2 breast histopathologists. Cases with a complete pathologic response were excluded.

Results: 48 cases with pretreatment and post treatment predictive markers were identified $(\mathrm{n}=48)$. ER status changed post neoadjuvant treatment in 2 of 48 cases $(4.2 \%)$. 39 of 48 cases $(81.2 \%)$ were ER positive pre treatment and 37 of 48 cases $(77.1 \%)$ were ER positive post treatment. PgR status changed post treatment in $27.1 \%$ of cases. 35 of 48 cases $(72.9 \%)$ were $\mathrm{PgR}$ positive pre treatment and 23 of 48 cases $(47.9 \%)$ were PgR positive post treatment. 1 case which was $\mathrm{PgR}$ negative pre treatment was PgR positive post treatment. 15 of 48 cases $(31.2 \%)$ were HER2 positive pre treatment. A change in HER2 status post treatment was observed in 4 cases $(8.3 \%)$. Of these, 2 cases changed from HER2 negative to HER2 positive and 2 cases changed from HER2 positive to HER2 negative.

Conclusions: ER, PgR, HER2 profiles changed post neoadjuvant treatment in a minority of breast carcinoma cases in this study. This post treatment change was observed most frequently for PgR. Possible reasons for the profile changes observed include tumour heterogeneity, core biopsy sampling and/or clonal selection during treatment. Re-testing hormone receptor and HER2 status post-neoadjuvant treatment is important as post treatment predictive marker changes can be clinically relevant and may guide further treatment choices in patients who have not achieved complete pathologic response.

\section{Risk of Developing Breast Cancer in Patients with Flat Epithelial} Atypia (FEA) in Benign Breast Biopsies

SM Said, W Rizzo, AC Degnim, RD Frank, RA Vierkant, MH Frost, LC Hartmann, A Nassar, DW Visscher. Mayo Clinic, Rochester, MN; Mayo Clinic, Jacksonville, FL.

Background: Little is known about the (long term) breast cancer risk for patients with FEA detected in benign biopsies

Design: Original slides from13381 women with benign breast biopsies from 1967 to 2001 were retrospectively categorized into three histologic groups: non-proliferative (8449 patients, $63.1 \%$ ), proliferative disease without atypia $(4232,31.7 \%)$, atypical hyperplasia $(\mathrm{AH}$, subcategorized as $\mathrm{ADH}$ and $\mathrm{ALH})(700,5.2 \%)$ and for presence of FEA. Breast cancer events were obtained from medical records and questionnaires. Relative risks (RRs) were estimated by comparing the number of observed breast cancers with the number expected on the basis of the rates of breast cancer in the Iowa Surveillance, Epidemiology, and End Results registry.

Results: Biopsies from 328 women (2.5\%) had FEA: it was accompanied by AH in $168(51.2 \%)$. After a mean follow up of 13.5 years (range, 0.5 to 39.0$), 57(17.4 \%)$ patients with FEA developed breast cancer including 24 (42.1\%) with isolated FEA and $33(57.9 \%)$ with accompanying AH. In the 24 patients with isolated FEA, the cancer developed in the same breast containing FEA in 16 patients and contralaterally in 8 . The cancer was invasive in 15 , DCIS in 5 , and unknown in 4 . The median time from FEA diagnosis to cancer diagnosis was 9.4 years (range, 0.5 to 31.0 ). The RR of breast cancer was higher for women with FEA $(\mathrm{RR}=3.51 ; 95 \% \mathrm{CI} 2.66-4.54)$ compared to those without FEA ( $\mathrm{RR}=1.60 ; 95 \%$ CI 1.51-1.69), $(p<0.0001)$. Among women with $\mathrm{AH}$, the $\mathrm{RR}$ was similar for women who also had FEA $(\mathrm{RR}=4.38 ; 95 \% \mathrm{CI} 3.01-6.15)$ compared to those who did not $(\mathrm{RR}=4.21 ; 95 \% \mathrm{CI} 3.47-5.07)(p=0.8468)$. After excluding the $\mathrm{AH}$ cases, the RR for isolated FEA $(\mathrm{RR}=2.75 ; 95 \% \mathrm{CI} 1.76-4.10)$ was not significantly higher than proliferative disease without atyipa $(\mathrm{RR}=1.89 ; 95 \% \mathrm{CI} 1.72-2.07),(p=0.0891)$. Conclusions: Patients with FEA have a higher risk of progression to breast cancer than patients without FEA, but this is primarily driven by its association with $\mathrm{AH}$. In women with $\mathrm{AH}$, the additional presence of FEA does not increase breast cancer risk.
308 Comparative Study of Ki67 Labeling Index in HER2 Amplified Versus Non-Amplified Estrogen Receptor Positive Invasive Carcinoma of the Breast

D Samrao, R Bhargava, D Dabbs, B Clark. Magee-Womens Hospital of UPMC, Pittsburgh, PA.

Background: The amplification/overexpression of ERBB2/HER2 (HER2+) in invasive breast carcinoma (IBC) is a surrogate marker for tumor aggressiveness and worse prognosis when compared to HER2 non-amplified (HER2-) cases. The mechanisms of action of HER2 are many, one of which is promotion of tumor cell proliferation. Thus, it is expected that HER2 + IBC would have a higher proliferation index than HER2- IBC when measured immunohistochemically (IHC) with the surrogate marker Ki67. The mechanism of action of anti-HER2 therapy is likely via antibody dependent cellular cytotoxicity; it may/may not specifically target cell proliferation. Therefore, additional chemotherapeutic drugs are added to trastuzumab which specifically target cell proliferation and result in improved tumor response.

Design: Due to dearth of studies on proliferation indices on ER+/HER2+ tumors, we studied Ki-67 proliferation index on $35 \mathrm{ER}+/ \mathrm{HER} 2+$ tumors. These tumors constitute $\sim 10 \%$ of all breast cancers. These $35 \mathrm{ER}+/ \mathrm{HER} 2+$ tumors were compared to a large pre-existing data set of $939 \mathrm{ER}+/ \mathrm{HER} 2$ negative tumors from the same time period at our institution. The mean Ki67 was calculated for both groups and the unpaired t-test was used to determine statistical significance for the difference.

Results: The mean Ki67 labeling index was $22 \%$ and $37 \%$ for ER+/HER2 negative and ER+/HER2+ cases respectively. The difference between the means was $15(95 \%$ confidence interval, 8.64 to 21.63 ). ER+/HER2+ cases show a statistically significant higher mean proliferation index than ER+/HER2 negative cases $(\mathrm{p}<0.0001)$.

Conclusions: Amplification of HER2 in IBC results in an increase in tumor cell proliferation as measured by Ki67. These findings support classification of ER+l HER2+ tumors as "luminal B" and likely explain why trastuzumab based combination chemotherapy is better than trastuzumab alone in this group of tumors. Interestingly, rare cases of ER+/HER2 + with low Ki67 were identified. These may represent a subgroup of tumors in which HER 2 is promoting oncogenesis via a novel mechanism or in which genetic modulation of the proliferation pathway is occurring. Further studies studying the prognosis and molecular profile of this subgroup of patients may help to identify novel targets for drug therapy.

309 Historadiologic Factors to Predict an Upgrade for Atypical Ductal Hyperplasia Diagnosed on an MRI-Guided Core Needle Biopsy: A Study of 46 Cases from Four Institutions

S Sanati, R Karabakhtsian, Z Li, MM Desouki, B Reig, P Kumar, X Chen, D Wang, X Huang, S Liu, T Khoury. Washington University, St. Louis, MO; Montefiore Medical Center, New York, NY; University of Pittsburgh Medical Center, Pittsburgh, NY; Vanderbilt University, Nashville, TN; Roswell Park Cancer Institute, Buffalo, NY.

Background: Recent studies propose that certain historadiologic findings could predict the upgrade for mammographically detected atypical ductal hyperplasia (ADH). Due to its rarity, little is known about the risk or the predictive factors for an upgrade for ADH diagnosed on MRI-guided core needle biopsies (CNB).

Design: We reviewed 785 MRI-guided CNB from four institutions over 7-years. The pathologic features of ADH were assessed including number of foci, size of largest focus, cumulative size of foci involved with $\mathrm{ADH}$, total number of cores, number of involved cores, and size of the biopsy needle. MRI findings including mass-enhancement (ME) vs. non-mass-enhancement (NME), and reason for MRI including high risk screening, staging, equivocal mammogram, or clinical suspicion were recorded. An upgrade was considered when the subsequent surgical excision specimen yielded invasive carcinoma (IC) or ductal carcinoma in situ (DCIS). Fisher exact test was used for statistical analysis. Results: There were 46 candidate cases, of which 11 (23.9\%) had upgrade. Table 1 illustrates the risk of upgrade for each variable. Our results indicated that the reason of MRI-guided biopsy was the only factor that significantly predicted an upgrade. Patients who underwent MRI examination for equivocal mammogram or for a clinical suspicion were significantly at higher risk for upgrade than when MRI was performed for high risk patients or staging, 9 of $28(32.1 \%)$ cases vs. 2 of $18(11.1 \%)$ cases, respectively $(\mathrm{p}=0.02)$.

Conclusions: The risk of upgrade is in the range of mammographically detected ADH. Therefore, ADH diagnosed on MRI-guided CNB should be surgically excised. The reason for performing MRI appears to be the only factor that significantly predicts the risk of upgrade.

\begin{tabular}{|c|c|c|}
\hline Variables(total) & Upgrade-No & Upgrade-Yes \\
\hline \multicolumn{3}{|l|}{ Pathology } \\
\hline \#Foci & $2(1-5)^{*}$ & $2(1-11)$ \\
\hline \#Cores & $11(2-16)^{*}$ & $12(5-12)$ \\
\hline \#Involved cores & $2(1-4)^{*}$ & $2(1-8)$ \\
\hline Largest focus size & $1.3(0.3-4)^{*}$ & $1.5(0.5-3)$ \\
\hline Cumulative size & $2(0.5-8.1)^{*}$ & $2(0.5-11)$ \\
\hline \multicolumn{3}{|l|}{ MRI } \\
\hline $\operatorname{Mass}(15)$ & $12(34)^{* *}$ & $3(27)$ \\
\hline Non-mass(31) & $23(66)^{* *}$ & $8(73)$ \\
\hline \multicolumn{3}{|l|}{ Reason for $\mathrm{MRI}^{\wedge}$} \\
\hline Equivocal mammogram(4) & $1(3)^{* *}$ & $3(27)$ \\
\hline Clinical Suspicion(24) & $18(51)^{* *}$ & $6(55)$ \\
\hline High risk(16) & $15(43)^{* *}$ & $1(9)$ \\
\hline Staging(2) & $1(3)^{* *}$ & 1(9) \\
\hline
\end{tabular}

*median and range; $* * \mathrm{~N}(\%) ;{ }^{\wedge} \mathrm{p}=0.02$ 
310 Comparison of the Novel Marker GATA3 vs Traditional Markers Mammaglobin and GCDFP15 in Surgical and Cytology Specimens of Metastatic Breast Carcinomas with Matched Primaries

AR Sangoi, B Shrestha, G Yang, O Mego, AH Beck. El Camino Hospital, Mountain View, CA; Cellmarque, Rocklin, CA; Harvard Medical School and Beth Israel Deaconess Medical Center, Boston, MA.

Background: Traditionally relied upon membranous/cytoplasmic markers mammaglobin and GCDFP15 show good specificity but often lack sensitivity and can be difficult to interpret in small tissue samples or those with high background staining. A comparative study of the novel nuclear marker GATA3 (expression typically restricted to breast and urothelial carcinomas) was thus undertaken.

Design: 137 cases of metastatic breast carcinoma of various subtype and 51 cases with available matched primary tumors were retrieved, including both surgical and cytology specimens. Patients with any other malignancies were excluded. One whole-slide section from each case was stained for monoclonal GATA3 (L50-823), monoclonal mammaglobin (31A5), and monoclonal GCDFP15 (EP1582Y). Staining intensity $(0-3+)$ and extent $(0-100 \%)$ were scored with an $\mathrm{H}$-score calculated (range $0-300)$ Results: Patient ages (all women) ranged from 31-98y (mean 63.5y). 109 cases were surgical specimens (43 biopsies, 66 resections) and 28 were cytology specimens. Staining results are listed in Table 1 .

Antibody sensitivity with minimum H-score cut-offs for a positive result in metastatic (M) and

\begin{tabular}{|c|c|c|c|}
\hline H-SCORE $(0-300)$ & GATA3 & GCDPF15 & MAMMAGLOBIN \\
\hline Any & $\mathrm{M}=96 \%{ }^{*} \mathrm{P}=94 \%$ & $\mathrm{M}=66 \% \mathrm{P}=82 \%$ & $\mathrm{M}=78 \% \mathrm{P}=88 \%$ \\
\hline 50 & $\mathrm{M}=93 \% * \mathrm{P}=94 \%{ }^{*}$ & $\mathrm{M}=39 \% \mathrm{P}=51 \%$ & $\mathrm{M}=48 \% \mathrm{P}=50 \%$ \\
\hline 100 & $\mathrm{M}=91 \%{ }^{*} \mathrm{P}=92 \%^{*}$ & $\mathrm{M}=26 \% \mathrm{P}=33 \%$ & $\mathrm{M}=28 \% \mathrm{P}=24 \%$ \\
\hline 150 & $\mathrm{M}=86 \% \%^{*} \mathrm{P}=90 \% *$ & $\mathrm{M}=21 \% \mathrm{P}=27 \%$ & $\mathrm{M}=20 \% \mathrm{P}=14 \%$ \\
\hline 200 & $\mathrm{M}=72 \%^{*} \mathrm{P}=82 \%^{*}$ & $\mathrm{M}=19 \% \mathrm{P}=18 \%$ & $\mathrm{M}=11 \% \mathrm{P}=4 \%$ \\
\hline 250 & $\mathrm{M}=64 \% * \mathrm{P}=75 \%{ }^{*}$ & $\mathrm{M}=15 \% \mathrm{P}=12 \%$ & $\mathrm{M}=7 \% \mathrm{P}=0 \%$ \\
\hline
\end{tabular}

* $=\mathrm{P}<0.0001$ for both GATA3 vs GCDPF 15 and GATA3 vs mammaglobin comparisons

For cases with matched primary and metastatic samples, we observed significantly stronger correlation between primary and metastatic GATA3 expression (Pearson's Correlation $=0.81[0.68-0.89])$ as compared with the primary to metastatic correlation of GCDFP15 (Pearson's correlation $=0.57[0.33-0.74]$ ) and as compared with the primary to metastatic correlation of mammaglobin (Pearson's correlation $=0.50[0.24$ 0.70]) (Both $\mathrm{P}<0.05)$.

Conclusions: The novel nuclear marker GATA3 stains a significantly higher proportion of both primary and metastatic breast carcinomas than GCDFP15 or mammaglobin, and the matched primary/metastasis expression of GATA3 is more consistent. GATA3 positivity is also stronger and more diffuse, helpful in cases with small tissue samples.

311 Total Tumor Load: Testing the Utility of This New Molecular Tool When Analysing the Axillary Dissection by Molecular Techniques

I Sansano, B Vieites, M Sancho, C Garcia, I Amendoeira, L Bernet, JM Perez, M Espinosa, I Rubio, S Ramon y Cajal, V Peg. HU Vall d'Hebron, Barcelona, Spain; HU Virgen del Rocío, Sevilla, Spain; HU Salamanca, Salamanca, Spain; HU Porto, Porto, Portugal; HU Lluís Alcanyís, Xàtiva, València, Spain.

Background: Despite the publication of the ACOSOG Z0011 trial, it has not been uniformly applied. Multiple studies have aimed to identify variables predictive of nonSLN metastases to select those patients who can be spared complete axillary lymph node dissection (ALND). In a previously published study we found that a new variable, the total tumoral load (TTL), defined as the amount of CK19 mRNA copies/ $\mu 1$ in all SLN assessed by the One Step Nucleic acid Amplification assay (OSNA) was predictive of non-SLN metastasis. However, the ALND was not analyzed with molecular techniques but with HE, and it is well known that histopathology underestimates the number of non-SLN. The aim of this study was to test the predictive value of TTL when using OSNA for both SLN and non-SLN evaluation.

Design: A cohort of 82 patients who underwent ALND after identification of SLN metastasis by the OSNA assay was included. These patients had histologically confirmed CK19 positive breast cancer, pT1-T3, clinically node-negative and with either conservative or radical surgery. None of these patients had metastatic disease, neoadjuvant therapy, or less than 10 axillary lymph nodes (including the SLN). We analysed all nodes by OSNA and recorded TTL and axillary status. For the statistical analyses we performed U Mann-Whitney test and ROC curve.

Results: 125 out of 131 SLN were positive with TTLs that ranged from 124 to 2E08 CK19 mRNA copies (mean 4,65E06). A total number of 1116 non-SLN was obtained, mean 13.7 (range 8-32). The molecular assay detected 61 macrometastases and 68 micrometastases. Regarding ALND, 52 out of 82 patients (63.4\%) were diagnosed as positive. We found statistically significant differences between TTL in positive and negative ALND $(\mathrm{p}=0,043)$. The ROC curve analyses showed an area under the curve $=0.64(\mathrm{p}=0,043)$. For the cut-off value of 15000 copies (proposed in our previous study) we obtained a negative predictive value $(\mathrm{NPV})=0.52$, positive predictive value (PPV) $=0.70$, sensitivity $=0.77$, and specificity $=0.43$.

Conclusions: These results show that when using the same methodology for analyzing SLN and non-SLN, TTL still predicts the status of the non-SLN. This new tool can then be useful when deciding axillary clearance in early breast cancer patients.

$312 \quad$ Atypical Ductal Hyperplasia Diagnosed on Core Needle Biopsy with Excisional Biopsy Follow-Up of a 10 Year Period, Does the Size of the Needle Biopsy Affect Management?

$K$ Sarah, C Wells, SK Apple. UCLA David Geffen School of Medicine, Los Angeles, CA. Background: Upgraded lesions are defined as a lesion initially diagnosed as atypical ductal hyperplasia (ADH) on core needle biopsy (CNB) but found to have ductal carcinoma in situ (DCIS) or invasive carcinoma at excisional biopsy (EB) and have been reported ranging from $4-54 \%$. Vacuum-assisted 9 gauge needle was used from
2005 in our institution and prior to that, the most commonly used was 14-gauge needles. We postulated that upgrade from $\mathrm{ADH}$ would decrease by using large gauge (9 to 12) needles and sought to find out if our hypothesis is true.

Design: We identified a total of $276 \mathrm{CNB}$ diagnosed as ADH over a 10 year period from 2003-2013 retrospectively. We excluded other atypia diagnoses such as flat epithelial atypia (FEA) alone, reactive atypia, $\mathrm{ADH}$ associated with papillary lesions and ADH associated with invasive carcinoma and/or ductal carcinoma in situ (DCIS) on CNB. We included ADH seen with other associated lesions such as FEA, atypical lobular hyperplasia, lobular carcinoma in situ (ALH/LCIS), and ADH bordering on low grade DCIS. The parameters included are BIRADS, imaging findings, and size of needles used. Almost all CNB samples from 2003-2005 were 14-gauge needle, and more than $90 \%$ of the cases after 2005 were larger size (9- or 12-) needles, under ultrasound guidance or stereotactic biopsies.

Results: We identified 57 ADH cases (20\%) from 2003-2005 versus 219 ADH cases $(80 \%)$ from 2006-2012. On EB, DCIS and/or invasive carcinoma were found in 67/276 cases (24\% upgrading). Residual ADH and any atypia (such as FEA, LCIS/ALH) were found in 94 cases (34\%). Benign breast tissue without any residual atypia is seen 56 cases $(21 \%)$ and loss of follow-up after CNB was 59 cases (21\%). From 2003-2005, upgrades were $25 \%$ when compared to $24 \%$ from 2006-2012 when larger gauge needles are used for CNB.

Upgrades on EB after ADH on CNB, 2003 to 2005

\begin{tabular}{|l|l|l|}
\hline 2003 & 2004 & 2005 \\
\hline $2 / 17=12 \%$ & $4 / 18=22 \%$ \\
\hline
\end{tabular}

Upgrades on EB after ADH on CNB, 2006 to 2012

\begin{tabular}{||l||l|l||l|l||l|l|}
\hline 2006 & 2007 & 2008 & 2009 & 2010 & 2011 & 2012 \\
\hline $4 / 23=17 \%$ & $4 / 26=15 \%$ & $11 / 34=32 \%$ & $15 / 32=47 \%$ & $2 / 12=17 \%$ & $11 / 42=26 \%$ & $6 / 50=12 \%$ \\
\hline
\end{tabular}

Conclusions: Our total upgrade rate of $24 \%$ is comparable to previous studies. Increasing the needle gauge size on CNB did not affect the rate of upgrading on EB. Residual ADH, FEA, LCIS/ALH and/or upgrades were found in $58 \%$ of cases of ADH thus $\mathrm{EB}$ is recommended in all cases of ADH diagnosed by CNB.

313 Comprehensive Genomic Profiling of Metaplastic Breast Carcinoma by Next Generation Sequencing Reveals Frequent Actionable Genomic Abnormalities and New Routes to Targeted Therapies

$C E$ Sheehan, SS Badve, $K$ Wang, AB Boguniewicz, GA Otto, G Palmer, $R$ Yelenksy, $D$ Lipson, SA Ali, D Morosini, VA Miller, PJ Stephens, JS Ross. Albany Medical College, Albany, NY; Indiana University School of Medicine, Indianapolis, IN; Foundation Medicine Inc, Cambridge, MA.

Background: Metaplastic breast carcinoma (MPBC) accounts for less than $1 \%$ of breast cancers, but carries a significantly worse prognosis. We hypothesized that genomic profiling with a next generation sequencing (NGS)-based assay could identify genomic alterations (GA) that could identify potential targeted therapy for this disease.

Design: Hybridization capture of 3,769 exons from 236 cancer-related genes and 47 introns of 19 genes commonly rearranged in cancer was applied to $>50 \mathrm{ng}$ of DNA extracted from $20 \mathrm{MPBC}$ FFPE specimens and sequenced to high (average 833X), uniform coverage. Genomic alterations (base substitutions, small indels, rearrangements and copy number alterations) were determined and then reported for these patient samples. Actionable GA were defined as those identifying anti-cancer drugs on the market or in registered clinical trials (CT).

Results: The 20 patients had an average age of 61.5 years (range $42-86$ years). There were $9(45 \%)$ squamous, $9(45 \%)$ chondroid and $2(10 \%)$ spindle cell MPBC all of which were high grade. One MPBC was stage II, 2 were stage III and 17 were stage IV. Of the $16 \mathrm{MPBC}$ with available results, $6 \%$ were $\mathrm{ER}+, 13 \%$ were $\mathrm{PR}+, 88 \%$ were triple - and $100 \%$ were HER2- by IHC or FISH testing. A total of 93 GA were identified, with at least one alteration identified in all cases for an average of $4.65 \mathrm{GA}$ per tumor. Nineteen (95\%) MPBC cases harbored a total of 36 actionable GA with an average of 1.8 actionable GA per tumor. This result is similar to a series of 273 routine breast cancer evaluated by the same assay, where $246(87 \%)$ had actionable GA. The most common unactionable GA were alterations in TP53 (80\%), MYC (30\%), MLL2 (25\%) and KDM6A (10\%). The most common actionable GA were mutations, amplifications and homozygous deletions of PIK3CA (40\%), PTEN (25\%) and CDKN2A/B (20\%), EGFR $(10 \%)$ and HRAS (10\%) with AKT3, BRCA2, CCND1, CCND2, CCNE1, CDK4, FBXW7, FGFR1, NF1, PIK3R1 and SRC altered in a single case. The MPBC ERBB2 negative status was identical to IHC/FISH results in $100 \%$ of cases.

Conclusions: Ninety-five percent of MPBC harbored at least one actionable GA. Given the limited treatment options and poor prognosis of metastatic MPBC, comprehensive NGS-based genomic profiling has the potential to identify new treatment paradigms and fulfill a previously unmet clinical need for this disease.

314 A Detailed Histologic Analysis of Prior Benign Biopsies in Women with Breast Cancer by Hormone Receptor Status

YM Sheinin, ME Sullivan, TA Thurow, A Mahajan, A Patel, IB Helenowski, SA Khan. Northwestern University Feinberg School of Medicine, Chicago, IL; University of Wisconsin-Madison, Madison, WI.

Background: Prior benign biopsies (BBx) are a risk factor in developing breast cancer (BC) and are used in calculations such as the Gail model. There are known benign lesions associated with hormone receptor (HR) positive $\mathrm{BC}$, but no definitive correlate exists for HR negative $\mathrm{BC}$. We performed a detailed histologic examination of prior $\mathrm{BBx}$ including looking for proposed precursors of HR- BC such as complex apocrine metaplasia (AM) and microglandular adenosis (MGA). We also performed a large analysis of $\mathrm{BC}$ patients matched by age and race to see if there was a different rate of $\mathrm{BBx}$ in women with $\mathrm{HR}+\mathrm{vs}$. HR- BC. 
Design: Our breast database was searched to identify women who had a BBx $>6$ months prior to being BC diagnosis. All available slides were obtained and reviewed by breast pathologists blinded to the HR status of the $\mathrm{BC}$. The presence or absence of 17 specific histologic features was recorded. Age at $\mathrm{BBx}$ and age at $\mathrm{BC}$ diagnosis was recorded. A separate search generated a case-control set of women with $\mathrm{HR}+$ or HR- BC matched by age and race. Their complete pathology history was reviewed and any prior $\mathrm{BBx}$ was noted.

Results: 144 patients with $\mathrm{BC}$ and a prior $\mathrm{BBx}$ were identified; HR status was unknown in $11 \mathrm{BC}$. There were $119(89 \%) \mathrm{HR}+\mathrm{BC}$ and $14(11 \%)$ HR- BC. No significant difference in a specific histologic feature or in BBx category between $\mathrm{HR}+\mathrm{BC}$ and HR- $B C$ was found $(\mathrm{p}=0.54)$. MGA was not identified in any $B B x$. No AM was identified in $72 \%$ of HR- and complex AM was present in $14 \% \mathrm{HR}$ - vs $21 \% \mathrm{HR}+$. No significant difference was identified looking at average age, multiplicity and average interval. HR+ $\mathrm{BC}$ was diagnosed in the same breast in $55 \%$ of patients while HR- was in only $36 \%$. In our analysis of 757 matched pairs of women with BC, 40 in the HR+ group had a prior $\mathrm{BBx}$ while there were only 26 in the HR- group, a significant difference $(\mathrm{p}<0.0001)$. Conclusions: In our study, patients who go on to develop HR- BC have similar pathology in prior BBx as those who develop $\mathrm{HR}+\mathrm{BC}$. These results support the idea that there may not be a benign non-obligate precursor identifiable by routine histology for HR$\mathrm{BC}$. The incidence of prior BBx in women with HR- BC was significantly less than in women with $\mathrm{HR}+\mathrm{BC}$ matched by age and race. These results suggest that any risk associated with BBx may be limited to the development of HR+ BC.

\section{Analysis of Breast Cancer Cases with Polysomy 17}

$N$ Sidhu, $N$ Bures, $H$ Yang. University of Calgary, Calgary, AB, Canada.

Background: Human Epidermal Growth Factor Receptor 2 (HER2), a prognostic and predictive marker in breast cancer, is reported to be amplified or overexpressed in approximately $20 \%$ of cases. At our institution, HER 2 status is initially assessed by immunohistochemistry (IHC). Equivocal cases are retested by silver in situ hybridization (SISH). The results are determined by the ratio of average gene copy numbers of HER2 and chromosome 17 (HER2/CEP17) in cases of HER2 copy numbers between 4 and 6 . Based on local consensus, the cases with HER 2 copies $<4$ are regarded as NEGATIVE and HER 2 copies $>6$ as POSITIVE regardless of HER2/CEP17 ratio. The effects of increased CEP17 copy number (so called polysomy 17) on the final HER2 status and clinical management as well as the pathological characteristics of such cases are poorly understood and are investigated in this study.

Design: All new breast cancer cases that had HER2 IHC and subsequent SISH performed at our institution for the past 14 months were reviewed. Polysomy 17 cases were identified by the presence of $\geq 3$ average CEP17 gene copies per nucleus. The demographics, pathologic characteristics, average HER2 and CEP17 signals per nucleus, and the final reported HER2 gene amplification results were analyzed.

Results: Of 401 cases with HER2 SISH performed, 80 cases were identified as polysomy $17(20 \%)$. The patients were between 32-90 years of age (average 61 ) and 79 were women. The majority of cases were invasive ductal carcinomas (IDC) $(75 / 80 ; 93.8 \%)$, ER and PR positive (64/80; 80\%), and MBR grade III (49/80; 61.2\%). Lymphovascular invasion (LVI) was identified in 28/78 (35.9\%) cases, and 33/70 (47.1\%) cases were positive for lymph node metastases. Percentage of cases with HER2 signals per nucleus $<4$ was $42.5 \%$, 4-6 was $47.5 \%$ and $>6$ was $10 \%$. 61 out of 80 cases had 3-4 CEP 17 signals per nucleus $(76.2 \%)$. Cases with 4-6 HER2 copies were reported by HER2/ CEP 17 ratio and the 38 such cases all had NEGATIVE reports.

Conclusions: Polysomy 17 resulted in negative HER2 reporting in all breast cancer cases with 4-6 HER2 gene copies per nucleus. At our institution, these patients do not routinely receive trastuzumab-based therapy. Future follow up studies are planned to use alternative probes SMS, RARA, and p53 to determine if these cases are truly polysomic and clinically significant.

\section{6 miRNA-Mediated Anti-Metastatic Pathway Induction by Kallikrein 5 Over-Expression in Breast Cancer}

KG Sidiropoulos, NM White, A Bui, P Boulos, QC Ding, G Sotiropoulou, G Pampalakis, H Khella, GM Yousef. University of Toronto, Toronto, ON, Canada; St. Michael's Hospital, Toronto, ON, Canada; University of Patras, Rion-Patras, Patras, Greece. Background: Kallikrein 5 (KLK-5) is a serine protease that has been demonstrated to be down-regulated in breast cancer and other cancers.

Design: In order to understand the role of KLK5 in breast cancer pathogenesis, we examined miRNA and mRNA arrays for differential expression changes in both a stable KLK5 breast cancer cell line (MDA-MB-231) and publically available data from over 700 breast cancer patients.

Results: We identified 25 up-regulated and 60 downregulated miRNAs with KLK5 expression. These included up-regulated miRNAs: miR-183-5p, miR-206; and downregulated miRNAs: miR-935, mir-519a-5p, and miR-23b-5p. We confirmed that KLK5 over-expression down-regulated Drosha, Dicer, Exportin5, Ago 1 and Ago 2 of miRNA biogenesis pathway. Pathway analysis of the miRNA gene targets with KLK5 overexpression demonstrated KEGG pathways enrichment in cell-cell interaction, MAPK signaling, apoptosis and 553 pathways. KLK5 expression caused up-regulation of a set of miRNAs determined to be enriched in ECM-receptor interaction pathways. These gene targets were confirmed by analysis of mRNA array data, qRT-PCR, and miRNA inhibitors. The data demonstrated a reduction in cell to matrix adhesion molecules including integrins such as beta-1 (ITGB1), and reduced ECM protease expression with KLK 5 expression. We analyzed a sub-set of 16 breast cancer patients which had a change in KLK5, miR-183-5p and ITGB1 expression of at least $1 \mathrm{z}$-score. The data demonstrated a trend in which high expression of KLK5 and miR-183-5p was correlated with low ITGB1 expression and a basal-like breast cancer subtype with no metastatic patients. Conversely, lower expression of KLK 5 and miR-183-5p was correlated with higher ITGB1 expression and luminal breast cancers. Two patients with the lowest level of KLK 5 were correlated with confirmed breast cancer metastasis independent of breast molecular subtype. An analysis of about 500 breast cancer patients indicated KLK5 expression is significantly reduced in aggressive Luminal B breast cancers. A trend of increasing expression of KLK5 was found from Luminal A, Her2-neu, to Basal-like. Conclusions: KLK 5 potentiates down-regulation of multiple metastamirs through reduced miRNA biogenesis. Furthermore, KLK5 expression is positively correlated with distant metastasis-free breast cancers subtypes, with promotion of anti-metastatic miRNAs to inhibit migration and invasion of breast cancer cells.

317 Does Accurate Assessment of (Low) Hormone Receptor Status Require Dual Testing?

B Singh, NZiguridis, SM Butler, F Jamshidian, DB Cherbavaz, AP Sing, FL Baehner. NYU Langone Medical Center, New York, NY; Genomic Health, Inc, Redwood City, CA.

Background: Prognosis and treatment (Tx) for patients (pts) with early stage breast cancer (EBC) depend in part, on the status of HR expression. The 21-gene assay (Oncotype $\mathrm{DX}\left({ }^{\circledR}\right)$ is the only genomic test validated for both risk of distant recurrence and prediction of chemotherapy benefit in $\mathrm{ER}+$ pts. A formal interaction between ER expression by Oncotype DX assay and tamoxifen benefit was shown in NSABP B-14. Pts with ER(-) disease have a worse prognosis, do not respond to hormonal Tx (HT). Thus, accurate assessment of HR status is critical. Currently ASCO/CAP guidelines define pts with $>1 \%$ ER/PR staining as '+ ' for HR. Consequently, more pts are being treated with HT, but may not actually benefit. A study evaluating ER expression by IHC and RT-PCR was undertaken in consecutive EBC pts with either (-) or low + ER at a single center.

Design: ER(-) ( $<1 \%$ positive cells $)$ and ER low $+(1-10 \%$ positive cells $)$ by IHC pts were identified at NYU School of Medicine. HR assessment used standard IHC methods (SP1, 1E2, 4B5, Ventana). HR mRNA assessment used the 21-gene assay and reference-normalized expression measurements ranging from 0 to $15 \mathrm{C}_{\mathrm{T}}$ units, where each 1-unit increase reflects an $\sim 2$-fold increase in RNA. The validated $\mathrm{C}_{\mathrm{T}}$ thresholds for $(+)$ for the HR markers were used: ER, 6.5, PR, 5.5.

Results: Of 140 IHC evaluable samples, 106 (76\%) were ER(-), 34 (24\%) were ER low+, $116(83 \%) \mathrm{PR}(-)$, and $24(17 \%) \mathrm{PR}+$. Pts included: $69.3 \% \geq 50 \mathrm{yrs} ; 83.6 \%$ poorly differentiated; $31.2 \%$ had $>1$ node; $34.3 \%$ were $>2.0 \mathrm{~cm}$. The cross classification of ER by RT-PCR vs IHC is in the Table. IHC ER(-) cases had a $93.3 \%$ negative agreement (PNA) and IHC ER low + cases had $38.2 \%$ positive agreement (PPA). ER expression $\left(\mathrm{C}_{\mathrm{T}}\right.$ units) ranged from 2.8 to 8.3 for IHC (-) cases and 3.4 to 9.2 for $\mathrm{IHC}$ low + cases. Conclusions: This cohort of ER(-) or low+ EBC cases had high PNA (93\%) between IHC and RT-PCR for (-) ER expression; but, only $40 \%$ of ER low+ cases by IHC were positive by RT-PCR. Many of the discordant cases were near the RT-PCR cutpoint of 6.5 and $\sim 10 \%$ were either strongly ER+ or strongly ER(-) by RT-PCR suggesting misclassification by IHC. Thus, in many IHC ER low + cases there may be little or no benefit from the addition of HT. Accurate assessment of ER is critically important to ensure the best Tx. These data suggest that cases with low + ER by IHC represent an 'equivocal' category and dual testing may better clarify their ER status.

\begin{tabular}{|l|l|l|l|}
\hline ER Status & IHC \\
\hline RT-PCR & - & Low + & Total \\
\hline- & $99(93.3 \%)$ & $21(61.7 \%)$ & $120(85.7 \%)$ \\
\hline+ & $7(6.6 \%)$ & $13(38.2 \%)$ & $20(143 \%)$ \\
\hline Total & $106(100 \%)$ & $34(100 \%)$ & $140(100 \%)$ \\
\hline
\end{tabular}

318 Interfere with Routine Ancillary Test Results

$D$ Smith, $N$ Massoll, S Sharma, S Korourian. University of Arkansas for Medical Sciences, Little Rock, AR.

Background: Evaluation of mastectomy specimens for establishing an accurate pathological stage for breast cancer remains a challenge. Some of the radiographic imaging modalities are very helpful for identifying small lesions; however, these instruments are costly and not available at all institutions. We use a simple method of rapid chilling the mastectomy specimen at $-80^{\circ} \mathrm{C}$ for 20 to 30 minutes. This process allows the tissue to be thinly and evenly sliced. Thin slices are invaluable for recognizing small foci of invasion in specimens with a large area of involvement by ductal carcinoma in situ, and for recognizing multifocal and multicentric lesions. Studies have suggested that cold ischemia would cause inaccurate ancillary test results. We intend to show rapid chilling does not alter the ancillary test results and allows for more accurate gross evaluation.

Design: At our institution essentially $100 \%$ of breast resections follow an initial core biopsy performed at our facility or an outside institution. Ancillary studies (including hormone receptors and HER2 by IHC, and HER2 FISH) are routinely performed on the initial core biopsy; however, all initial results are verified on subsequent resections. Pathology files were reviewed to identify mastectomy cases with invasive carcinomas that have undergone rapid chilling during gross exam from 2010-2012. The results of the original ancillary tests were compared to the results on the mastectomy specimens performed at our facility.

Results: A total of 165 mastectomy cases with invasive carcinoma were identified. Fifty of these patients had initial core biopsies at an outside institution. A total of 36 patients had prior ancillary tests that were repeated on the mastectomy samples. These patients were divided into four categories. Group 1, hormone receptor positive and HER2 negative, 23 patients; group 2, hormone receptor positive and HER2 positive: two patients; group 3 , hormone receptor negative and HER2 positive: two patients; group 4, triple negative cases: 7 patients. We also identified two patients who had HER2 at the borderline level by FISH. When comparing the initial ancillary test results with the mastectomy results, all cases were concordant (100\%). 
Conclusions: Our results show rapid chilling of mastectomy tissue does not interfere with IHC for hormone receptors or HER2/neu, or with HER2/neu FISH. This quick chilling method would allow for a more complete gross examination of breast tissue, which might improve accuracy in pathological staging of breast cancer.

319 Metastases

A Soni, Z Ren, O Hameed, GP Siegal, S Wei. University of Alabama at Birmingham, Birmingham, AL.

Background: About $90 \%$ of $\mathrm{BC}$ mortality is due to metastases that are resistant to adjuvant therapies. The distant organs to which $\mathrm{BC}$ preferentially metastasize are of clinical and biological importance, and are closely related to the patients' survival outcomes. Previous studies have identified differentially expressed genes in BCs with organ-specific relapse. In this study, we explored the relationship between the common sites of distant metastases and the clinicopathological factors of the primary tumors in a large cohort of advanced staged patients.

Design: The tumor registry of the authors' institution was searched to identify BCs with associated distant organ metastases. The clinicopathological characteristics were recorded. BC subtypes were classified into luminal (ER+ and/or PR+), HER2 (ER-/ PR-/HER2+) or triple negative (TNBC). The luminal and TNBC subtypes were not further subdivided.

Results: Of all BC patients diagnosed from 1997 to 2010, 531 had distant metastases. Of these, 390 had single organ relapse and 141 had multi-organ involvement. The most common sites were bone ( $48 \%)$, liver $(27 \%)$, lung $(23 \%)$, central nervous system (CNS; $17 \%$ ) and pleura (7\%). While age, race, tumor type, size, and the number of positive nodes were not associated with relapse to any of these organs, the histologic grade was significantly correlated to CNS metastasis $(\mathrm{P}=.0004)$ but not other sites. Bone relapse was more frequently found for the luminal subtype $(\mathrm{P}<.0001)$ but less than expected in TNBC $(\mathrm{P}=.02)$. Liver metastasis was more frequently observed in the HER2 subtype $(\mathrm{P}=.01)$. Lung and pleural metastases were both commonly seen in TNBC $(\mathrm{P}<.0001$ and $\mathrm{P}=0.02$, respectively). Non-luminal subtypes were more commonly found in CNS $(\mathrm{P}=.009)$ and lung $(\mathrm{P}<.0001)$ relapsed patients. Further, the luminal subtype was strongly correlated $(\mathrm{P}=.0005)$, and HER 2 and TNBC were both inversely $(\mathrm{P}=.03$ and .02 , respectively) associated with bone-only metastasis $(\mathrm{N}=154)$ in the group of $\mathrm{BCs}$ with bone relapse, while the HER2 subtype remained significant for liver-only relapse $(\mathrm{N}=51 ; \mathrm{P}=.004)$ in $\mathrm{BCs}$ with liver metastasis.

Conclusions: $\mathrm{BC}$ subtypes seemingly differ in their ability to metastasize to distal organs, thus further delineating the earlier speculation regarding $\mathrm{BC}$ organotropism. This could potentially be utilized in determining the appropriate survey regimen for follow-up of patients with non-metastatic disease. Developing a conceptual framework for the intrinsic properties of $\mathrm{BC}$ cells with organ-specific metastatic potential, along with defining the host organ microenvironment, will be instrumental in guiding efforts to pursue personalized medicine.

320 Lobular Neoplasia Found on Core Needle Biopsy Should Be Excised: Upgrade Rates with Radiologic-Pathologic Considerations

MJ Stehura, H Gilmore, PE Bomeisl. University Hospitals Case Medical Center, Cleveland, $\mathrm{OH}$

Background: Lobular neoplasia (LN) encompasses both atypical lobular hyperplasia (ALH) and lobular carcinoma in situ (LCIS). As a mammographically occult lesion, the incidence of LN is difficult to determine. With increasing use of MRI, the incidence of LN may be rising. Controversy exists over the management of core needle biopsies (CNB) when LN is the most significant diagnosis. Several studies have looked at upgrade rates of $\mathrm{LN}$ in CNB to more significant lesions, and results vary from $1-30 \%$. However, many of these studies do not consider radiologic-pathologic concordance. Design: Surgical pathology records from 2007-13 were searched for CNB with LN. The imaging, radiologic-pathologic correlation, and pathologic diagnoses were recorded and compared. A case was considered upgraded on excision if ductal carcinoma in situ (DCIS) or invasive carcinoma (IC) was identified.

Results: In total, 146 cases of LN on CNB were identified. 111 cases were excluded because of concurrent ipsilateral DCIS or IC. Of the 35 remaining cases, 29 had follow-up excision. Of these cases, 13 (44\%) were upgraded on excision. In 7 (54\%) cases, the radiologic-pathologic findings were felt to be discordant. In the other 6 $(46 \%)$ cases, the LN was upgraded to a more significant lesion despite the apparent radiologic-pathologic concordance.

\begin{tabular}{|c|c|c|c|c|c|}
\hline 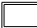 & Imaging & Imaging type & CNB Dx & Excision Dx & CNB Discordance \\
\hline 1 & Distortion/Calc & Mammogram & LCIS , SA & $\mathrm{IC}$ & $\mathrm{N}$ \\
\hline 2 & Enhancement & MRI & LCIS, FCC & ILC & $\mathrm{N}$ \\
\hline 3 & Mass & Mammogram & LCIS, FCC & ILC & $\mathrm{Y}$ \\
\hline 4 & Mass & Mammogram & ALH, FA & DCIS & $\mathrm{N}$ \\
\hline 5 & Mass & Mammogram & LCIS, FA & DCIS & $\mathrm{N}$ \\
\hline 6 & Mass & Mammogram & ALH & ILC, IC & $\mathrm{Y}$ \\
\hline 7 & Mass & MRI & ALH, UDH & IDC, DCIS & $\mathrm{Y}$ \\
\hline 8 & Mass & MRI & LCIS & ILC & $\mathrm{Y}$ \\
\hline 9 & Mass & Mammogram & LCIS & IDC, DCIS & $\mathrm{Y}$ \\
\hline 10 & Mass & Mammogram & LCIS & DCIS & $\mathrm{Y}$ \\
\hline 11 & Mass & Mammogram & LCIS, FA & Phyllodes & $\mathrm{N}$ \\
\hline 12 & Mass & Mammogram & ALH & IDC & $\mathrm{Y}$ \\
\hline 13 & Mass & Mammogram & ALH, ADH, RS & DCIS & $\mathrm{N}$ \\
\hline
\end{tabular}

Summary of cases upgraded from $\mathrm{LN}$ with radiologic characteristics. $(\mathrm{FA}=$ fibroadenoma, $\mathrm{FCC}=$ fibrocystic changes, $\mathrm{SA}=$ sclerosing adenosis, $\mathrm{UDH}=$ usual ductal hyperplasia, $\mathrm{RS}=$ radial scar

Conclusions: We identified an upgrade rate from $\mathrm{LN}$ to a more significant lesion (DCIS or IC) in $44 \%$ of the cases. Many of these CNBs were felt to be discordant and would have been referred to excision regardless of the LN, but many had benign findings in addition to the LN that could be considered radiographically concordant.
Based on these findings, we hesitate to recommend that diagnosis of $\mathrm{LN}$ on $\mathrm{CNB}$ be followed clinically even when there seems to be radiologic-pathologic concordance as the upgrade rate may be substantial.

321 Comparison of Oncotype DX Recurrence Scores in Caucasian and African-American Women

M Stehura, C Thompson, H Gilmore, P Bomeisl. University Hospitals Case Medical Center and Case Western Reserve University, Cleveland, $\mathrm{OH}$

Background: Oncotype $\mathrm{DX}^{\mathrm{TM}}$ is a commonly used 21 gene assay that predicts the benefit of chemotherapy for node-negative patients with all types and grades of estrogen receptor-positive breast cancer (BC). Results are used by clinicians to determine which patients may benefit from adjuvant chemotherapy. It is well documented that AfricanAmerican (AA) women often have higher grade breast cancer and present at earlier ages. Racial differences in Oncotype DX score have not been well characterized.

Design: Pathology reports were searched for all Oncotype $\mathrm{DX}^{\mathrm{TM}}$ reports from a 5 year period. The corresponding original pathology reports and patient demographics were reviewed to determine the patient's age, race, tumor histologic subtype, size, grade, and ER/PR/HER2 status. The pathologic features were compared with the Oncotype $\mathrm{DX}^{\mathrm{TM}}$ report data. Differences by race were assessed using a chi-square test or Fisher exact test for categorical variables. A standard t-test was used for continuous variables, and all p-values $<0.05$ were considered statistically significant.

Results: A total of 184 patients with Oncotype DX scores were identified. Of these, 152 were Caucasian and 32 were AA. The women ranged in age from 25 to 80 years old with an average age of 58.2 years $(\mathrm{SD}=10.8)$. The tumors ranged in size from $0.4-7.5$ $\mathrm{cm}$ with an average size of $1.98 \mathrm{~cm}(\mathrm{SD}=1.16)$. When stratified by race, the average age of both groups were similar, as were the distribution of grade and percentage positive of ER and PR ( $>0.05)$. In this sample, the average Oncotype score was only slightly higher in AA women than in Caucasian women and not statistically significant. When the groups were sorted into recurrence risk categories, more AA women fell into the high risk group ( $16 \%$ vs. $10 \%$ of Caucasians). However, this was also not statistically significant. Our data did show that the AA women did have a larger average tumor size (mean 2.4 $\mathrm{cm}$, SD $(1.5 \mathrm{~cm})$ vs. $1.9 \mathrm{~cm}$, SD (1.1) in Caucasians, $\mathrm{p}=0.016)$. A higher percentage of grade 3 tumors was observed in AA women but was not statistically significant

Conclusions: In this series, there was not a substantial difference in the Oncotype scores among AA women compared to Caucasian women. This series contained only one-fifth the number of AA women when compared to Caucasian women which is consistent with the patient population in this institution. Despite the lack of difference in Oncotype scores, AA women did have a significantly larger average tumor size at presentation.

322 Does Morphological and Molecular Heterogeneity of Individual Tumor Foci in Multiple Breast Carcinoma Influence the Treatment? A Study of 155 Cases with Analysis of $\mathbf{4 6 3}$ Tumor Foci

S Stolnicu, C Moldovan, A Eniu, M Boros. University of Medicine and Pharmacy Targu Mures, Targu Mures, Romania; Oncologic Institute Cluj Napoca, Cluj Napoca, Romania. Background: The aim of this study is to evaluate biomarker immunophenotypic heterogeneity between separate tumor foci of multiple breast carcinomas, its correlation with morphological features and tumor grade, and its influence on the treatment.

Design: 155 invasive multiple breast carcinomas were retrospectively analyzed over a 6-year period (2007-2012), and the expression of estrogen (ER) and progesterone (PR) receptors, ki-67 proliferative index, HER-2 expression, morphological subtype and tumor grading were analyzed in each tumor focus.

Results: We found mismatches in immunohistochemical features in $71(53.78 \%)$ out of 132 patients with similar histology and in $13(56.52 \%)$ out of 23 cases with different histology. When analyzing mismatches in ER and PR status together, in $4(23.52 \%)$ out of 17 cases one of the tumor foci was ER or PR positive, while the index tumor did not express either marker. The most numerous mismatches (45 cases, $29.03 \%$ ) concerned the proliferative index; in 14 cases $(9.03 \%)$ the additional focus had a higher index than the main focus, and in 9 of these cases the additional focus displayed a 3 histological grade. Mismatches in HER-2 status appeared in 25 (16.12\%) cases. The histological type of the additional foci was different from the index tumor in $23(14.83 \%)$ cases. Conclusions: Assessment of all tumor foci would have determined $19(12.25 \%)$ cases to receive different adjuvant treatments compared to what would have been indicated if only the biological status of the largest primary tumor was assessed. We strongly recommend assessing and reporting each tumor focus independently.

323 Value of Ki67 Proliferation Index in Identifying Patients with HighRisk Breast Cancer

J Stone, A Ordobazari, B Hunt, B Behmaram. Medical College of Wisconsin, Milwaukee, WI.

Background: Indicators of poor prognosis in invasive mammary carcinoma include large size, nodal and distant metastasis, estrogen and progesterone receptor negativity, HER2/neu amplification, angiolymphatic invasion, BRCA1/2 positivity, and high histologic grade. More recently, Ki67 proliferation index has been shown to be a prognostic factor in invasive carcinoma of the breast, with high proliferation exhibiting shorter relapse free interval and decreased overall survival. Moreover, Ki67 is being requested as part of the routine breast biomarker panel to serve as a prognostic indicator and help guide treatment decisions. Does adding Ki67 proliferation index provide additional prognostic information not provided by routine clinical and pathologic information?

Design: Biopsies from 100 cases of invasive mammary carcinoma were selected at random from the institutional database. Eighty seven had sufficient tissue for further analysis. Each case was stained with MIB-1 antibody and Ki67 proliferation index was assessed by visual estimation. Cases of grade 1 and 2 ductal and lobular carcinomas 
with an elevated Ki67 proliferation index, defined as being $>14 \%$, were selected. The patient's clinical and pathologic data were reviewed to assess for high risk features. Results: Forty-four cases out of 87 were grade $1(51 \%)$, twenty-six were grade $2(30 \%)$ and seventeen were grade $3(19 \%)$. Seven grade 1 carcinomas had an elevated Ki67. All of these patients had additional high-risk features, including HER2/neu amplification, triple negative biomarkers, lymph node positivity, and progesterone receptor negativity. Ten grade 2 carcinomas had an elevated Ki67. All of these patients also had additional high-risk features including HER2/neu amplification, triple negative biomarkers, tumor recurrence, lymph node positivity, BRCA1 positivity, pleomorphic lobular carcinoma, and progesterone receptor negativity. All grade 3 carcinomas had an elevated Ki67. Conclusions: All of our study patients with grade 1 or 2 invasive mammary carcinoma and elevated Ki-67 proliferation had additional poor prognostic features at presentation. These results suggest that in most cases of low to intermediate grade carcinoma, routine assessment of Ki67 proliferation index adds little prognostic information beyond that already provided by standard clinical and pathologic information.

\section{HER2 Heterogeneity by FISH in Breast Cancers and Matched}

\section{Lymph Node Metastases: How Different Are They?}

CJ Suarez, RA Schmidt, SM Dintzis, MH Rendi, KC Jensen, RB West, KH Allison. Stanford University School of Medicine, Stanford, CA; University of Washington Medical Center, Seattle, WA.

Background: In 2009, recommendations for reporting minor populations (5-50\%) of cells with HER2 amplification by fluorescence in situ hybridization (FISH) were published ("HER2 heterogeneous cases"). However, there is little data available about the significance of these minor intermixed HER2 positive cell populations. We examined the percentage of HER2 amplified cells by FISH in primary breast cancers and their matched lymph node (LN) metastases.

Design: FISH for HER2 was performed on 68 untreated, unselected primary breast cancers and their matched lymph node metastases. The percentage of individual cells with a HER2:CEP17 ratio $\geq 2.0$ (original clinical trials cutoff utilized for analysis of individual cells) was compared between the primaries and their matched LN metastases. Least-squared linear regression analysis was performed.

Results: By $2007 \mathrm{CAP} / \mathrm{ASCO}$ criteria, $80.9 \%$ of primaries were considered negative (mean ratio $<1.8$ ), $14.7 \%$ were amplified (mean ratio $>2.2$ ) and only $4.4 \%$ were equivocal (mean ratio 1.8-2.2). Between primaries and their matched $\mathrm{LN}$ metastases, the percentage of cells with ratios $\geq 2.0$ increased in $41.2 \%$ of cases, decreased in $32.4 \%$, and did not vary in $26.5 \%$. The average percent change was $8.25 \%(\mathrm{SD}=0.095)$. No cases went from an overall HER2 non-amplified status in the primary to an amplified result in the LN metastasis. $73.5 \%$ of the primaries and $75 \%$ of the $\mathrm{LN}$ metastases demonstrated HER 2 heterogeneity (between $5-50 \%$ neoplastic cells with ratios $>2.0$ ). In this subset of cases, the change in percentage of cells with ratios $\geq 2.0$ from primary to metastasis did not differ from the entire set (average percent change $=8.17 \%, \mathrm{SD}=0.072$ ). All heterogeneous cases had non-clustered (intermixed) heterogeneity. Overall, a positive linear correlation for the percentage of cells with ratios $\geq 2.0$ was observed between the primaries and their matched LN metastases with a proportional relationship near $1: 1\left(r^{2}=0.811, p<0.0001\right.$; slope $=0.899,95 \%$ CI: 0.772- 1.027).

Conclusions: These results suggest that in an unselected series of breast cancers with LN metastases at initial diagnosis and predominantly non-equivocal HER2 FISH results, the degree of HER2 genetic heterogeneity in the primary closely reflects that in the metastasis. We plan to expand this study to include more cases near the threshold for positive to determine if cases with borderline results behave differently.

\section{Clinical and Immunohistochemical Differences in Subtypes of} Lobular Carcinoma In Situ

ME Sullivan, E Neuschler, V Parini, B Susnik. Northwestern University, Chicago, IL; Piper Breast Center, Minneapolis, MN.

Background: Lobular carcinoma in situ with necrosis (LCIS-N) and pleomorphic lobular carcinoma in situ (LCIS-P) can be differentiated from classic lobular carcinoma in situ (LCIS-C) morphologically by the presence of high grade nuclear atypia, marked acinar distention and comedo necrosis. Although they are known to be aggressive versions of LCIS, differences between LCIS-P and LCIS-N are not well delineated and are they are often combined into a vague poorly understood group of LCIS variants. Design: The pathology database was searched to identify breast core biopsy (CB) with LCIS-P and/or LCIS-N and confirmed by review and E-cadherin. A series of consecutively diagnosed CB with LCIS-C was reviewed for comparison. Data including radiologic presentation, menopausal status and family or personal history of breast cancer was collected. A panel of immunostains was performed on all LCIS-P and N: estrogen receptor (ER), progesterone receptor (PR), HER2, p53 and Ki-67. Excision pathology was also obtained.

Results: 35 LCIS-P (20 LCIS only; 15 with invasion), 29 LCIS-N (21 LCIS only; 8 with invasion) and 63 LCIS-C CB were identified. LCIS-P was more common in postmenopausal women. Both LCIS-P and LCIS-N presented as a BI-RADS $4 \mathrm{C} / 5$ lesion significantly more often than LCIS-C $(\mathrm{p}<0.0001)$.

Clinical and Imaging Characteristics

\begin{tabular}{|c|c|c|c|c|}
\hline & $\|$ Age & Postmenopausal & BI-RADS 4C/5 & Family Hx \\
\hline LCIS-P & 57 & $80 \%$ & $39 \%$ & $41 \%$ \\
\hline LCIS-N & 55 & $70 \%$ & $23 \%$ & $51 \%$ \\
\hline LCIS-C & 54 & $63 \%$ & $6 \%$ & $37 \%$ \\
\hline
\end{tabular}

LCIS-P had unfavorable biomarkers (ER- or HER2+) more often than LCIS-N. 14.7\% of LCIS-P was ER- while no ER- LCIS-N cases were identified. Both LCIS-P and LCIS-N occasionally showed HER2 positivity. LCIS-P more often had a Ki-67>10\% compared to LCIS-N

\begin{tabular}{|c|c|c|c|c|}
\hline \multicolumn{5}{|c|}{ Unfavorab } \\
\hline & ER- & HER2+ & $\mathrm{p} 53+$ & Ki-67>10\% \\
\hline LCIS-P & $5 / 35(14 \%)$ & $5 / 34(15 \%)$ & $7 / 33(21 \%)$ & $14 / 33(42 \%)$ \\
\hline LCIS-N & $0 / 29$ & $3 / 25(12 \%)$ & $1 / 25(4 \%)$ & $6 / 25(24 \%)$ \\
\hline p value & 0.058 & 0.99 & 0.12 & 0.17 \\
\hline
\end{tabular}

No significant difference in panel results was demonstrated between CB with LCIS vs. CB with invasion. LCIS-P was upgraded at excision in 2 cases $(2 / 19 ; 10.5 \%)$ both to pT1mi. LCIS-N was upgraded in 6 cases $(6 / 17 ; 35.2 \%), 3$ with microinvasion and 3 with pT1 tumors.

Conclusions: All the LCIS patients are similar in age and family history. However, both LCIS-P and LCIS-N significantly differ in their imaging presentation. LCIS-N has more morphologic overlap with LCIS-C, sharing bland cytologic features and strong positivity for ER. This differs from LCIS-P which had ER- cases and was highly proliferative. Despite a more favorable IHC profile and classic cytology, LCIS-N was upgraded $3 \mathrm{x}$ more often than LCIS-P at excision.

\section{IGFR1 Silencing Induces Mesenchymal to Epithelial Transition in} Breast Cell Lines

HC Sullivan, MJ Swadley, FE Castaneda-Valladares, A Adams, LT Smith, R O'Regan, A Marcus, G Bedolla, C Cohen, GM Oprea-Ilies. Emory University, Atlanta, GA; ISSEMYM-Tlalnepantla, Tlalnepantla, Mexico.

Background: Gene expression analysis has defined luminal A and B, HER2 enriched and triple negative subtypes of breast cancer. Triple negative tumors (TNT) do not express the currently used prognostic and predictive markers ER, PR and HER2. They represent up to $20 \%$ of breast cancers in certain ethnic groups, showing a worse outcome and lack of targeted therapy. Insulin growth factor (IGFR) is instrumental in breast cancer cell proliferation, adhesion, invasion and migration. In-vitro it has an inhibitory effect on the metastatic properties of mesenchymal TNT cell lines including upregulation of epithelial markers and downregulation of some mesenchymal inducers. Our findings indicate that inhibitors of IGF1R exhibit promising anti-cancer activity in TNBC cells of mesenchymal origin by triggering mesenchymal to epitelial transition (MET), inducing cell death, and reducing invasive and metastatic capacity. While IGF1R expression is detected at higher levels in ER positive (ERP) vs. ER negative (ERN) tumors, active (phosphorylated-IGFR1)(p-IGFR) is detected at significantly higher levels in ERN tumors. The aim of the study was to determine if our findings in the TNT cell lines in vitro are translated in breast tissue tumors evaluated by immunohistochemical (IHC) methods. IGFR1 IHC was correlated with epithelia to mesenchymal transition markers. Design: Tissue microarrays (TMAs) of TNT were constructed with two $1 \mathrm{~mm}$ representative cores from each tumor and were immunostained with monoclonal antibodies to IGFR1, e-cadherin and vimentin. The intensity of staining (on a scale from 1 to 3 ) and the percentage of positive tumor cells were semiquantitavely scored. These data along with age, race, tumor grade and stage were entered in an excel spreadsheet for statical analysis.

Results: TNTs from 115 tumors were analyzed of which 61 were from African American patients and $\mathbf{4 4}$ form Caucasians patients. The age at diagnosis ranged from 24-90 years. There were no statically differences in age, race, tumor grade and stage in these tumors. IGFR showed strong statistically significant positive correlation with e-cadherin expression $(\mathrm{p}=0.0009)$ while no statistically significant correlation was found with vimentin expression.

Conclusions: 1 . Studies assessing correlations between active, p-IGFR with e-cadherin would be a better approach to evaluate the metastatic properties, particularly in TNT. 2. Targeted anti-IGFR agents could represent promising agents in management of this subset of breast cancers.

327 Quantitative Gene Expression by RT-PCR in the Tubular, Cribriform, Mucinous, and Papillary Histologic Subtypes of Invasive Breast Cancer

V Tan, FL Baehner, C Yoshizawa, JM Anderson, S Shak. Genomic Health, Inc, Redwood City, CA.

Background: ER + special histologic breast cancer subtypes are prognostically significant. Here we report the special histologic subtypes of ER+ breast carcinoma $\&$ associated patterns of observed gene expression as measured by the 21 gene Oncotype DX assay.

Design: All tumors from 6/1/04-5/31/12 were included in analyses. Central path used WHO criteria. Ductal NOS (DC), tubular (TC), cribriform (CC), mucinous (MC) \& papillary (PC) carcinomas were included. Quantitative expression of 16 cancer related genes was measured on a scale from 2 to 15 (relative to reference genes) where a 1 unit increment is associated with $\sim 2$-fold change in expression. Recurrence Score (RS) was calculated as published. Descriptive stats for the RS \& individual genes [ER, $\mathrm{PR}$, invasion gene group (IGG) \& proliferation gene group (PGG)] were obtained. Comparisons of means were adjusted to control the overall error rate under any complete or partial null hypothesis.

Results: DC accounted for $95.1 \%$, TC $0.7 \%$, CC $0.3 \%$, MC $3.1 \%$ and PC $0.7 \%$ of 246,555 cases. Mean age, mean RS, and proportion of ER+/PR-phenotype are shown in the table below. Mean age among histologic subtypes was statistically significant. For all types a wide continuous range of RS was noted. DC had the highest mean RS, followed in decreasing order by MC, TC, CC \& PC. PC had the highest ER \& PC \& $\mathrm{CC}$ the highest PR. TC had the lowest ER. ER was not different between CC and MC but PR was. The proportion with ER+/PR-phenotype was different among the subtypes: TC $(8.1 \%) \&$ CC $(7.1 \%)$ had the lowest incidence whereas MC $(12.9 \%) \&$ PC $(10.3 \%)$ were more similar to DC (14.1\%). TC had lowest PGG expression. MC \& PC had lower IGG expression compared to other subtypes.

Conclusions: The special subtypes had a wide continuous range of RS: DC had the highest mean RS, followed in decreasing order by MC, TC, CC and PC. PC had the highest ER \& PC \& CC the highest PR. TC had the lowest ER (may reflect submission 
bias). ER was not different between $\mathrm{CC}$ and $\mathrm{MC}$ but PR was. The proportion with ER+/ PR- phenotype was different among the subtypes: TC \& CC had the lowest incidence, MC \& PC were more similar to DC. TC had the lowest PGG expression. MC and PC had lower IGG expression.

\begin{tabular}{|l|l|l||l|l|l||}
\hline Histologic Subtype & N & \% of cases & Mean Age & RS Mean & ER+/ER- \\
\hline Ductal carcinoma, NOS & 234,518 & 95.1 & 58 & 19.7 & $14.1 \%$ \\
\hline Tubular carcinoma & 1,831 & 0.7 & 54 & 15.0 & $8.1 \%$ \\
\hline Cribriform carcinoma (IBC) & 827 & 0.3 & 56 & 12.6 & $7.1 \%$ \\
\hline Mucinous carcinoma(IBC) & 7,682 & 3.1 & 59 & 16.0 & $12.9 \%$ \\
\hline Papillary carcinoma(IBC) & 1,697 & 0.7 & 62 & 10.6 & $10.3 \%$ \\
\hline \hline
\end{tabular}

\section{Screen-Detected Versus Symptomatic Breast Ductal Carcinoma} In Situ: Comparison of Clinicopathological and Immunophenotypical Characteristics

PH Tan, V Koh, J Lim, AA Thike, GH Ho, J Wong, S Thilagaratnam. Singapore General Hospital, Singapore, Singapore; National Cancer Centre Singapore, Singapore, Singapore; Health Promotion Board, Singapore, Singapore.

Background: Breast cancer is the commonest malignancy in Singapore women, and has remained so since the inception of the Singapore Cancer Registry in 1968. Ductal carcinoma in situ (DCIS) is the putative precursor of the majority of invasive breast cancers. The efficacy of the Singapore breast screening pilot project in detecting early stage breast cancer led to the launch of the National Breast Screening programme, BreastScreen Singapore (BSS), in January 2002. In this study, we compared clinicopathological and immunohistochemical characteristics, as well as clinical outcome, between screen-detected and symptomatic DCIS.

Design: The study cohort comprised 1254 cases of DCIS diagnosed at the Department of Pathology, Singapore General Hospital from 1994 to 2010. Data for screen-detected DCIS were retrieved from the Health Promotion Board and symptomatic cases from hospital records. Clinicopathological parameters were reviewed and follow-up obtained. Immunohistochemistry for estrogen receptor (ER), progesterone receptor (PR), HER2, CK14, EGFR was performed using standard sections. Comparison was carried out between the two groups. A p value $<0.05$ defined statistical significance.

Results: Among 1254 cases, 620 (49.4\%) were screen-detected and 634 (50.6\%) were symptomatic DCIS. Screen-detected cases were smaller $(\mathrm{p}<0.001)$, of lower nuclear grade $(\mathrm{p}=0.002)$, more frequently expressed $\mathrm{ER}(\mathrm{p}<0.001)$ with less EGFR positivity $(\mathrm{p}=0.023)$. Triple negative and HER2 phenotypes were more common in symptomatic DCIS $(p=0.001)$. Mean and median follow up was 7 and 6 years respectively with maximum follow up of 18 years. Patients who were screen-detected experienced fewer recurrences $(\mathrm{p}<0.001)$ and deaths $(\mathrm{p}=0.013)$, while those with symptomatic DCIS disclosed poorer DFS (hazard ratio, 1.599; 95\% confidence intervals, 1.036-2.466; $\mathrm{p}=0.034)$. A trend for better overall survival $(\mathrm{p}=0.064)$ was observed in screen-detected patients.

Conclusions: Our data indicate a favorable outcome of screen-detected DCIS patients confirming the role of BreastScreen Singapore in early identification of this disease.

329 Rational Manual and Automated Scoring Thresholds for the Immunohistochemical Detection of TP53 Missense Mutations in Human Breast Carcinomas

N Taylor, N Feinberg, B Midkiff, J Geradts. University of North Carolina, Chapel Hill, NC; Duke University Medical Center, Durham, NC.

Background: Missense mutations in the tumor suppressor gene TP53 are among the most common molecular abnormalities in human breast cancer. They are associated with accumulation of $\mathrm{p} 53$ protein in the nuclei of tumor cells, which produces increased staining in immunohistochemical (IHC) assays. Most previous studies have used arbitrary cutoffs to identify positive cases. The current study was performed to determine optimal thresholds for manual and automated $\mathrm{p} 53$ scores.

Design: Whole tissue sections from the Carolina Breast Cancer Study (CBCS) that were previously evaluated for $\mathrm{p} 53$ positivity by manual IHC scoring and whose $\mathrm{p} 53$ mutational status was known were utilized to assess the performance of an Aperio automated nuclear algorithm. Slides were available for 564 tumors that had been immunostained for p53 and scored for average nuclear staining intensity and percent cells positive. Receiver operating characteristic curve (ROC) analysis was performed using SAS ver. 9.3 to determine optimal cutoffs for average staining intensity and percent cells positive to distinguish between tumors with and without a missense mutation.

Results: Regarding the manual IHC scores, cutoff points for the IHC detection of p53 missense mutations were established for percent positive tumor cells $(\geq 50 \%)$, average nuclear staining intensity (at least moderate) and modified $\mathrm{H}$-score $(\geq 120)$, with AUCs from 0.894 to 0.931 , sensitivities from $84.1 \%$ to $90.3 \%$, specificities from $88.1 \%$ to $88.4 \%$, and odds ratios from 42.0 to 58.6 . A moderate or strong average staining intensity identified 95 of 113 missense mutations and 50 of 421 tumors without such mutations (accuracy $87.3 \%$ ). Due to the variable dilutional effect of benign cells in the tissue sections, no cutoffs were determined for percent positive cells or $\mathrm{H}$-score in the automated analysis. An average automated staining intensity of at least moderate nuclear staining had an AUC of 0.84 , a sensitivity of $73.5 \%$, a specificity of $93.1 \%$, and an odds ratio of 37.5. It detected 83 of 113 missense mutations and 29 of 422 tumors without such mutations (accuracy $89.0 \%$ ). The concordance in average intensity scores between manual and automated reads was $91.3 \%$.

Conclusions: An automated scoring algorithm for the IHC detection of TP 53 missense mutations correlated well with manual scores. Thresholds were statistically determined for both manual and automated IHC scores that predicted the presence of a p53 missense mutation in human breast carcinomas with high accuracy.
330 Cancer Stem Cell Markers in Triple Negative Breast Cancer: Correlation with Clinicopathological Parameters and Clinical Outcome AA Thike, NM Chew, SS Ong, WY Taq, SY Tan, H Li, PH Tan. Singapore General Hospital, Singapore, Singapore.

Background: Triple negative (TN) breast cancers, defined by the absence of estrogen receptor, progesterone receptor and c-erbB2 (HER2) expression, have more aggressive clinical behavior and poorer prognosis, and are reported to possess a higher proportion of cancer stem cells. Tumors with stem cell properties are able to self-renew, harbor a higher cancer recurrence rate and are more resistant to therapy. This study investigates the presence of cancer stem cells (CSC) in a large series of TN invasive breast cancers in our institution, comparing immunohistochemical protein expression of various CSC phenotypes in relation to clinicopathological parameters and outcome.

Design: The cohort comprised 715 TN breast cancers diagnosed from 1994 to 2011 at the Department of Pathology, Singapore General Hospital. Antibodies to basal markers (CK14, 34ßE12, EGFR) and CSC markers (CD44, CD24 and ALDH1A1) were applied to sections cut from tissue microarray blocks, using the streptavidin-biotin method. Positive biomarker expression was defined as staining of $1 \%$ or more of tumor cells, with cytoplasmic membrane staining for CD44 and CD24, and cytoplasmic decoration for ALDH1A1. Disease free survival (DFS) and overall survival (OS) were defined as time from diagnosis to recurrence or death respectively, and correlated with protein immunohistochemical expression. A $p$ value $<0.05$ defined statistical significance.

Results: Expression of CD44, ALDH1A1, CD44+CD24-, CD44+CD24-ALDH1A1+, CD44+ALDH1A1+ was observed in $363(51 \%), 123(17 \%), 295(41 \%), 66(9 \%)$, and 84 $(12 \%)$ tumors respectively. Histologic grade was significantly associated with CD44+ $(\mathrm{p}=0.009), \mathrm{ALDH} 1 \mathrm{~A} 1+(\mathrm{p}=0.010), \mathrm{CD} 44+\mathrm{ALDH} 1 \mathrm{~A} 1+(\mathrm{p}=0.010)$ and CD44+CD24ALDH1A1+ $(p=0.050)$ tumors. CK14, EGFR and $34 \beta E 12$ confirmed $85 \%$ of cases to be basal-like and there was significant association between basal-like TN breast cancers and CD44+ $(\mathrm{p}<0.001)$ and CD44+CD24- tumors $(\mathrm{p}=0.002)$. Mean and median follow up was 94 and 83 months respectively, with maximum follow of 220 months. On multivariate analysis, enrichment of CD44 in the tumors was associated with poorer overall survival (hazard ratio, $1.398 ; 95 \%$ confidence intervals, $1.019-1.919 ; \mathrm{p}=0.0380$ ) especially within the first 5 years of diagnosis. No survival correlation was observed with other marker combinations.

Conclusions: Our study demonstrates that enrichment of CD44 in TN breast cancers is significantly associated with a poorer clinical outcome. The detection of CD44 expression in TN breast cancers may be an attractive marker that can provide new insights for further therapeutic paradigms.

\section{Androgen Receptor and Bcl-2 Expression in Ductal Carcinoma In}

\section{Situ of the Breast}

TA Thurow, J Martinez de Ubago, Y Sheinin, A Mahajan, S Rohan, KP Siziopikou. Northwestern University, Feinberg School of Medicine, Chicago, IL.

Background: The role of estrogen receptor (ER) and progesterone receptor (PR) as markers of predictive and prognostic significance in breast cancer is well established. $\mathrm{Bcl}-2$, the prototypic antiapoptosis gene, is also known to be expressed in hormonally driven breast carcinomas. Recently, studies have shown that androgen receptor (AR) plays an important role in invasive breast carcinoma. However, the role of AR and Bcl-2 in ductal carcinoma in situ (DCIS) is less well understood. In this study, we evaluated the expression of AR and Bcl-2 in DCIS and correlated the results with molecular and histopathologic characteristics of the DCIS subtypes.

Design: 42 cases of pure DCIS treated with surgical excision at Northwestern Memorial Hospital (2008-2011) were identified. Tissue microarrays were constructed with 3 cores from each case to account for tumor heterogeneity. Pathologic tumor characteristics of architectural subtypes, nuclear grade, tumor size, and presence of microcalcifications were evaluated. Our immunohistochemical marker profile consisted of ER, PR, HER2, $\mathrm{Ki}-67, \mathrm{AR}$, and Bcl-2.

Results: 20 of 42 cases were classified as Luminal A(ER+/Her2-), 5 as Luminal B (ER+/ Her2+ or ER+/Her2-/Ki67 high), 11 as Her2 (ER-/Her2+), and 3 as triple-negative (ER-/ Her2-). AR expression showed no difference between the four molecular DCIS subtypes. In contrast, Bcl-2 was expressed in 21/25 (84\%) of ER positive (luminal A and B) DCIS cases but in only $6 / 14(42 \%)$ of the ER negative (HER2 positive and triple negative) cases $(\mathrm{p}=0.0003) .12 / 12(100 \%)$ of our low and intermediate nuclear grade DCIS cases expressed the AR+/Bcl-2+ phenotype; while 15/15 (100\%) AR+/Bcl-2- cases showed high nuclear grade, $73 \%$ of which belonged in the HER 2 molecular subgroup. Finally, 2/2 cases with AR-/Bcl-2- phenotype also had high nuclear grade. No correlation was seen with age, architectural subtypes, tumor size, or presence of calcifications.

Conclusions: 1. AR does not appear to have a differential expression among the molecular subtypes of DCIS, unlike what has been reported for invasive carcinoma of the breast. 2. Bcl-2 expression is more likely to be seen in the Luminal A and B subtypes and less common in the HER2 and triple negative subtypes. 3. DCIS cases with $\mathrm{AR}+/ \mathrm{Bcl}-2$ - phenotype are high grade DCIS cases and belong to the HER2 molecular subtype. Our findings add to the understanding of the molecular mechanisms that drive the different molecular subtypes of DCIS that have the potential to lead to new more effective preventive and treatment strategies in DCIS.

332 Differential Expression of Tiam1 and Osteopontin in TumorAssociated Fibroblasts Contributes to Breast Cancer Invasion

$X$ Tian, KXu, SP Naber, RJ Buchsbaum. Tufts Medical Center, Boston, MA.

Background: We have found that silencing of Tiam1, a Rac exchange factor, in tumorassociated fibroblasts induces increased invasion and metastasis in cancer cells and a mouse model of human breast cancer (Xu K et al. Oncogene 29:6533, 2010). We have 
also found that changes in Tiam1 expression in fibroblast induce converse changes in fibroblast expression and secretion of osteopontin (OPN), a glycoprotein associated with tumor invasion and metastasis (Liu et al. J Cell Sci 125:376, 2012).

Design: To further understand the clinical significance of Tiam1 and OPN in tumor invasion, we collected 20 cases of invasive ductal carcinoma and 20 cases of high grade ductal carcinoma in situ (DCIS) of the breast. Tiam1 expression in the tumor-associated fibroblasts was assessed in invasive carcinoma and DCIS by immunohistochemical staining (IHC). OPN expression was also assessed by IHC in tumor-associated fibroblasts in the same samples. The underlying mechanism of Tiam 1 regulated tumor invasion was further investigated using a novel method for isolating human breast cancer cells out of 3D spheroid co-cultures containing mammary fibroblasts either knocking down the Tiam1 expression by shRNA techniques or over-expressing Tiam1. Results: The results showed that Tiam 1 expression was significantly decreased in fibroblasts associated with human invasive ductal carcinoma of breast, compared to fibroblasts associated with high-grade DCIS. The opposite pattern for OPN expression was observed. OPN expression was significantly increased in fibroblasts associated with invasive ductal breast carcinomas, compared to fibroblasts associated with high-grade DCIS. Furthermore, in three-dimensional (3D) spheroid co-cultures, human breast cancer cells co-cultured with Tiam1-deficient fibroblasts showed increased cancer cell invasion into matrix, while human breast cancer cells co-cultured with Tiam1-overexpressing fibroblasts showed decreased invasion into matrix.

Conclusions: These results suggest that regulated Tiam1 expression in breast cancerassociated fibroblasts modulates breast cancer invasion through regulation of fibroblast OPN secretion. By focusing on the most common type of breast cancer in woman, invasive ductal carcinoma, we demonstrate commensurate changes in fibroblast Tiam1 and OPN expression correlating with invasiveness, suggesting a promising mechanism for therapeutic targeting.

333 Metaplastic Breast Carcinomas: High Levels of EZH2 Are Associated with Tumor Stem Cells

KA Toy, Y Zhang, CG Kleer. University of Michigan, Ann Arbor, MI; University of California Davis, Sacramento, CA

Background: Metaplastic breast carcinoma is a subset of triple negative cancers with poor outcome and no targeted therapy, characterized by the presence of metaplastic components (e.g. spindle, squamous, and chondroid). Recent studies demonstrate that metaplastic, mesenchymal-like cells exhibit properties of cancer stem cells and heightened invasion. Overexpression of $\mathrm{EZH} 2$, a regulator of cell type identity, increases stem cell renewal and induces invasion. However, whether EZH2 is elevated in metaplastic carcinomas is unknown. In the present study we investigated the pattern and expression levels of EZH2 in different components of metaplastic carcinomas, and tested the hypothesis that EZH2 expression may be associated with the stem cell marker ALDH1, and with absence of the collagen receptor DDR1, which is a feature of increased invasiveness.

Design: In 27 primary metaplastic breast carcinomas we determined the expression of EZH2, ALDH1 and DDR1 proteins by standard immunoperoxidase (anti-EZH2, BD Biosciences, mouse monoclonal, Clone 11, 1:250; anti-ALDH-1, BD Biosciences, mouse monoclonal, clone 44, 1:5000; and anti-DDR1, Santa Cruz, rabbit polyclonal, Cat\# SC-532, 1:250). EZH2 was evaluated for intensity of reactivity based on a previously validated scoring system ( 1 to 4 ; EZH2 low $=$ scores $1-2$; EZH2 high $=$ scores 3-4). The presence of ALDH1 and DDR1 expression was considered positive, in each tumor component. Statistical analysis was performed by two tailed Fischer's exact test. Results: High EZH2 was positive in 23 of the 27 (85\%) metaplastic carcinomas but not in benign breast epithelium. EZH2 is expressed in the nucleus of spindle $(n=6$, $86 \%)$, squamous $(\mathrm{n}=13,100 \%)$ and chondroid $(\mathrm{n}=4,67 \%)$ components, in addition to the glandular areas. Of the 27 metaplastic carcinomas, high EZH2 expression in the metaplastic component was significantly associated with expression of the stem cell marker ALDH1 in $52 \%$ of cases $(\mathrm{p}=0.0063)$, and with negative DDR1 protein in $44 \%(\mathrm{p}=0.0003)$

Conclusions: We found first evidence that EZH2 is overexpressed in the spindle, chondroid and squamous components of metaplastic breast carcinomas, in association with expression of the stem cell marker, ALDH1, and negative DDR1. Future studies will need to address the role of EZH2 in the pathogenesis of metaplastic breast carcinomas, and the potential significance for prognosis and targeted treatment of this particular subset of aggressive tumors.

\section{Is Heterogeneity Important in Breast Cancer?}

$M$ Trippel, A Grogg, K Pfaltz, C Tapia. University of Bern, Bern, Switzerland.

Background: Tumor heterogeneity on the DNA-, RNA-, and protein-level has significant clinical relevance related to its apparent impact on therapy response. However, the significance of morphological tumor heterogeneity in the primary tumor as well as in the lymph node metastasis has earned little attention. We investigated a large number of primary breast cancers concerning morphological intra-tumoral heterogeneity and its correlation with lymph node status. Further, we evaluated the lymph node metastasis for heterogeneity.

Design: We evaluated 360 primary breast cancers and 162 corresponding lymph node metastasis from patients diagnosed at our institute between the years 2005-2011. The primary tumors and the positive lymph nodes were re-evaluated and classified according to the WHO classification, respectively. If more than one histological type was present the percentage of the amount of each histological type was estimated both in the primary and the lymph nodes. The TNM classification, the hormone receptor and her2-status as well as the proliferation fraction were taken from the pathology reports. Our results from the primary tumor were correlated with the lymph node status and the morphology of the lymph node metastasis.
Results: We found morphological intra-tumoral heterogeneity in 52 breast cancers $(14.4 \%)$. Heterogeneous breast cancer had significantly $(\mathrm{p}<0.009)$ more lymph node metastasis $(66 \%)$ compared to non-heterogeneous tumors $(50.8 \%)$. The following 8 histological types were observed in the heterogenous primary tumor: NST $n=43$, lobular $n=14$, micropapillary $n=12$, mucinous $n=7$, signet ring cell $n=3$, cribriform $\mathrm{n}=2$, tubular $\mathrm{n}=2$ and glycogen rich $\mathrm{n}=1$. A morphological heterogeneity in the lymph node metastasis was found in 9 cases with the following histological types: NST $=9$, micropapillary $=7$ and signet ring cell $=2$.

Conclusions: Our study could show that morphological intra-tumoral heterogeneity is a frequent event in breast cancer (14.4\%) especially in NST and lobular types (9.4\%). Heterogeneous breast cancers seem to be more aggressive with significantly greater incidence of lymph node metastasis $(\mathrm{p}<0.009)$. Therefore, we are convinced that any morphological intra-tumoral heterogeneity should be mentioned in the pathological report.

335 3D Ex Vivo Primary Breast Mammosphere Cultures Exhibit Similar Mutational Profiles as Original Tumors

A Vadodkar, VVaradan, K Miskimen, L Harris, H Gilmore. Case Comprehensive Cancer Center, Cleveland, $\mathrm{OH}$.

Background: Next generation deep sequencing of breast cancers (BC) has revealed intratumoral heterogeneity that may play a role in therapy resistance. Growing BC cells in 3D cultures termed mammospheres is one model system that has advantages over using standard cell lines in 2D tissue culture because it more closely recapitulates the true tumor environment. However, it is crucial to understand to what extent the 3D cultures recapitulate the clonal heterogeneity found in the original tumor

Design: Portions of discarded fresh BC tissue samples from 3 patients were frozen in OCT, formalin fixed and paraffin embedded (FFPE), and plated for mammospheres. Then, DNA was extracted from the OCT, FFPE, and mammospheres. The Qiagen GeneRead DNAseq Breast Cancer panel was used as it targets the coding and UTR regions of 20 most commonly mutated genes in BC. NGS was performed using Illumina MiSeq platform and variant calls were identified using GATK Unified Genotyper in Illumina BaseSpace. High confidence somatic mutations were identified by filtering likely germline SNPs using dbSNP while retaining variants present in the COSMIC database.

Results: We obtained an average of $2.6 \mathrm{M}$ reads per sample sequenced with $90 \%$ of reads aligning to within the target region of the Qiagen panel with a median coverage of 1284x. A high degree of concordance in somatic mutation calls in the OCT, FFPE and Mammosphere from all 3 tumors (T1, T22, and T13) was seen. In tumor T1, 6/7 somatic mutations found in OCT and FFPE samples were also in the mammosphere ( $86 \%$ concordance). A p53 mutation (p.K93N) was the only one not found in the mammosphere. Tumor T13 showed $86 \%(6 / 7)$ concordance across samples with a mutation in the 3' UTR of FBXW7 present only in the mammosphere. T22 had $60 \%$ concordance with a mutation in the PTEN 5' UTR seen only in the mammosphere and p53 mutation (p.C238Y) seen only in the OCT.

Figure 1: Concordance of somatic mutations across OCT, FFPE and Mammosphere
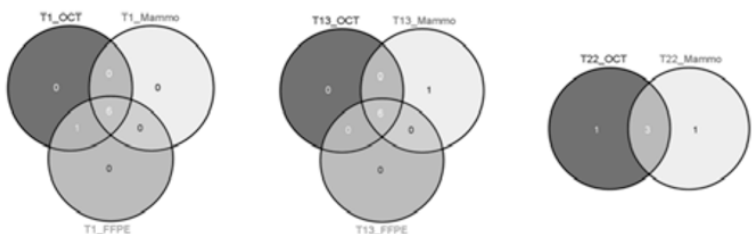

Conclusions: A high degree of concordance in somatic mutations between OCT, FFPE and mammosphere samples derived from the same patient tumor was seen. Occasional differences could be evidence of selection during the growth of the mammospheres and requires additional follow-up. This suggest that mammospheres are largely representative of tumor samples from patients and can be used as relevant ex vivo models.

336 Genetic Analysis of HER2 Equivocal Heterogeneous Tumors: How Genetic Categories Can Help in the Final Decision for HER2 Gene Status?

A Valent, M-C Mathieu, A Bernheim, L Arnould, G MacGrogan, J-M Guinebretiere, $F$ Chibon, F Penault-Llorca, M Lacroix-Triki. Gustave Roussy Cancer Campus, Villejuif, France; Centre G-F Leclerc, Dijon, France; Institut Bergonie, Bordeaux, France; Institut Curie, Saint-Cloud, France; Centre Jean Perrin, Clermont-Ferrand, France; Institut Claudius Regaud, Toulouse, France.

Background: Tumor heterogeneity is present in different malignancies including breast cancer (BC). HER2 genetic heterogeneity as assessed by Fluorescence in situ hybridization (FISH) is defined by coexistence of genetically variant tumor cell subpopulations in sample(s) of the same patient. Tumor heterogeneity can be observed either at the same site (HER2 gene status differs between cells of a single tumor) or at multisites (discordance between primary tumor and metastasis). The $H E R 2$ heterogeneity exists in all IHC categories, but seems to be more important in IHC HER2 2+ category. Design: Centralized HER2 FISH was performed in 50 invasive $\mathrm{BC}$ locally assessed as IHC $2+$ score and complex by FISH. These cases were heterogeneous tumors harboring additional genetic aberrations that made interpretation difficult (eg. chr 17 monosomy, HER2/CEP17 coamplification, CEP17 amplification alone). We focused on genetic categories and their detailed description. The ratio and mean HER2 and CEP17 copy numbers were calculated for each sample and all genetic clones were detailed.

Results: Out of $50 \mathrm{BC}, 10$ were amplified and 40 non-amplified. For 21 patients we could detect HER 2 amplified clone in 2 or 3 different samples and compare the proportion of 
amplified cells. $H E R 2$ non amplified tumors were also very heterogeneous, since we could detect 2 to 14 different genetic subpopulations within the same tumor. Altogether, 5 different genetic categories were detected in IHC $2+$ (amplified and non-amplified) tumors: A)disomy, B)monosomy (simple, or with gain or amplification), C)chromosome 17 "polysomy" (whole chromosome (rare) or segmental -more frequent), D)HER2 gain (1 to 4 more HER2 copies compared to CEP17), E)HER2 gene amplification (homogeneous or heterogeneous). We established the ratio for most of the cases except for: 1)monosomic cases, gain of 2-3 HER2 copies would "shift" non-amplified tumor to amplified, 2)CEP17 amplification, 3)HER2/CEP17 coamplification, 4)presence of clusters (hsr, accurate count impossible).

Conclusions: The existing ACSO/CAP guidelines are used in routine practice to establish $H E R 2$ gene status. However samples where the strict application of these rules is difficult do exist. In heterogeneous tumors, the percentage of major genetic clone together with ratio and mean $H E R 2$ copy number might be better criteria for accurate HER 2 status assessment.

337 Reflex ER/PR/Her2 Analysis of Breast Cancers in Needle Core Biopsy Specimens Significantly Increases Health Care Costs

CJ VandenBussche, A Cimino-Mathews, P Argani. The Johns Hopkins Medical Institutions, Baltimore, $\mathrm{MD}$

Background: ER/PR/Her2 are traditionally assessed on whole sections of excisional specimens (EXS) containing breast cancer. At our institution and many others, oncologists now ask that ER/PR/Her 2 be reflexively assessed on all core needle biopsies (CNB) showing invasive mammary carcinoma (IMC), so that neoadjuvant therapy can potentially be considered in all patients even though only a minority prove to be candidates. ER/PR/Her2 can be heterogeneous, and many recommend that negative results for any of these markers on small CNBs be repeated on larger EXS. The frequency and added cost of repeat ER/PR/Her2 EXS testing of those IMC with negative ER/PR/ Her2 CNB results has not been previously studied.

Design: We reviewed all CNBs showing IMC which had a subsequent excision performed at our institution over a 2 year period. We determined the number of cases in which $\mathrm{ER} / \mathrm{PR} / \mathrm{Her} 2 \mathrm{IHC}$ and Her2 FISH assays were negative on CNB, and whether or not they were repeated on EXS. The combination of the technical and professional fee was considered the assay's cost.

Results: We identified 202 CNB containing IMC from 200 patients. 27 (13\%) patients received neoadjuvant chemotherapy, while $7(3.5 \%)$ patients did not have residual IMC on follow up EXS, so in these cases obtaining ER/PR/Her2 results on CNB was necessary. Of the remaining 168 IMC, $29(17 \%)$ were ER negative and $41(24 \%)$ were PR negative on CNB. 162 IMC were tested for Her2 and $138(84.7 \%)$ were Her2 negative or equivocal on CNB. On EXS, ER and PR were repeated on 18 of $29(62 \%)$ ER negative IMCs and 23 of $41(56 \%)$ PR negative IMCs, respectively, while Her2 was repeated on only $38(25 \%)$ Her2 negative or equivocal IMCs of which $14(10 \%)$ underwent repeat FISH testing. Repeat testing resulted in a total increased cost of $\$ 10,783$. Had all negative tests been repeated as many recommend, the total increased cost was estimated to be $\$ 78,143$.

Conclusions: Reflex ER/PR/Her2 testing of IMC on CNB results in increased costs due to the need to repeat negative results on larger EXS. Extrapolating our institutional experience to the 230,000 new cases of IMC in the USA each year, repeat ER/PR/Her2 EXS testing of negative CNB results would result in an increased cost of $\$ 12,000,000$, and over $\$ 88,000,000$ if all negative tests had been repeated. CNB ER/PR/Her2 results guide neoadjuvant therapy in only a minority of cases. We recommend that $\mathrm{ER} / \mathrm{PR} / \mathrm{Her} 2$ not be reflexively ordered on all CNB specimens containing IMC, but instead be performed at the clinician's request for individual patients likely to receive neoadjuvant therapy.

338 Are Hyperplastic Enlarged Lobular Units (HELUs) in Benign Breast Biopsies Independent Predictors of Subsequent Breast Cancer Risk?

DW Visscher, TL Hoskin, FH Marlene, LC Hartmann, DC Radisky, AC Degnim. Mayo Clinic, Rochester, MN.

Background: Hyperplastic enlarged lobular units are a histological feature observed in some benign breast biopsies; they are characterized by hypercellularity and reduplication of misshapen, crowded acini with hyperchromatic cells. Some have speculated that HELUs may represent an early precursor of some breast carcinomas.

Design: We compared the overall presence, number and density (i.e. number/area) of HELU(s) in benign breast biopsies from 982 women who subsequently developed breast cancer to 1699 controls that were matched for age and year of biopsy (median follow up interval 19.3 years). Risk associations based on presence and densities of HELU(s) were analyzed with and without correction for overall histological categorization (i.e. non-proliferative vs proliferative vs atypical), family history of breast cancer and degree of lobular involution. The association of HELU measures with case-control status was assessed with conditional logistic regression.

Results: Overall, HELUs were uncommon in benign breast biopsies; $18 \%$ (490/2681) contained at least one HELU. Among these $490,50 \%$ had only 1 HELU and $50 \%$ had $>1$ HELU. Presence and density of HELU were both significantly associated with case status (i.e. breast cancer diagnosis after benign biopsy) in univariate analysis ( $\mathrm{p}=0.0001$ ). However, this association was confounded by a strong correlation of HELUs with atypical hyperplasia (AH); $17 \%$ of those with HELUs also had ADH and/or ALH as compared to $5 \%$ with $\mathrm{AH}$ among those with no HELUs $(\mathrm{p}<0.0001)$. The frequency of $\mathrm{AH}$ increased significantly with increasing numbers of HELU: AH was present in 5\% of samples with $0 \mathrm{HELU}, 12 \%$ of subjects with $1 \mathrm{HELU}$, and $21 \%$ of subjects with $>1$ HELU $(p<0.0001)$. Samples with high HELU density $\left(>0.83 \mathrm{HELU} / \mathrm{cm}^{2}\right.$, the median density among those with any HELU) had higher odds of cancer in follow up (OR
1.74 vs those without HELU, $95 \%$ CI: 1.33-2.28) in univariate analysis. However, this association was not significant (OR 1.18, 95\% CI: 0.86-1.62) after adjusting for other independent risk factors for breast cancer (overall histological category, degree of lobular involution, and family history).

Conclusions: HELU presence or density is strongly associated with synchronous presence of atypia but is not independently associated with increased risk for future breast cancer.

339 Aberrant Expression of Napsin A in Breast Carcinoma with Apocrine Features

T Vitkovski, S Chaudhary, M Nasim, M Esposito, T Bhuiya. Hofstra North Shore LIJ School of Medicine, Lake Success, NY; Mount Sinai School of Medicine, New York, NY. Background: Napsin A, initially proven to be a sensitive marker of lung adenocarcinoma, is now increasingly found to be less sensitive than originally thought. In our institution, recent incidental findings of 2 Napsin A positive breast carcinomas with apocrine features during workup of metastatic tumor in an axillary lymph node led to our further investigation of the incidence of Napsin A positivity in breast carcinomas, focusing on those with apocrine features. We aim to assess the frequency of Napsin A immunohistochemical staining in breast carcinoma with apocrine features together with TTF-1, GATA-3, and GCDFP.

Design: We collected 30 cases of primary invasive breast carcinoma with apocrine features, 4 cases of ductal carcinoma, 3 cases of lobular carcinoma, 1 case of secretory carcinoma, and 1 case of mucinous carcinoma. We performed immunohistochemistry with Napsin A, GATA-3, TTF-1, and GCDFP. GATA-3 has recently been reported to be positive in breast and urothelial carcinomas. Staining was scored as follows: weak: 1; moderate: 2; and strong: 3 . Percentage of tumor cells staining positive was measured. Tumors with staining in less than $5 \%$ of cells were regarded as negative.

Results: Thirty-nine cases were included in the study. Twenty-one of 30 cases $(70 \%)$ of apocrine breast carcinomas exhibited Napsin A expression as follows: strong in 5 cases $(17 \%)$, moderate in 6 cases $(20 \%)$, weak in 10 cases $(33 \%)$, and negative in 9 cases $(30 \%)$. The case of mucinous carcinoma showed moderate expression while the secretory carcinoma was negative. Three of four cases of ductal carcinoma were negative. Two of three cases of lobular carcinoma showed weak positivity. Overall, $64 \%$ of total cases exhibited some degree of Napsin A expression while only those with apocrine features demonstrated strong expression. GATA-3 was positive in 22 apocrine cases $(73 \%)$ and 6 non-apocrine cases $(67 \%)$. GCDFP was positive in 27 apocrine cases $(90 \%)$ and 6 non-apocrine cases $(67 \%)$. All 39 cases $(100 \%)$ were negative for TTF-1. Conclusions: In this sample size of 30 cases of breast carcinoma with apocrine features, $70 \%$ of cases stained for Napsin A including $37 \%$ which stained moderately to strongly positive. Our study shows that Napsin A is not a reliable marker in differentiating between breast carcinoma with apocrine features and lung adenocarcinoma. Positivity in metastatic lesions with apocrine features should not be interpreted as evidence of lung origin.

\section{Slug and IMP3 Are Co-Expressed in BRCA Positive Triple Negative} Breast Cancers

V Walavalkar, T Stockl, D Kandil, J Sotelo, S Samanta, A Mercurio, A Khan. University of Massachusetts Medical School, Worcester, MA.

Background: Slug (SNAI2) is a member of the Snail family of zinc finger transcription factors and is thought to play a role in epithelial to mesenchymal transition and induction of a 'basal-like' phenotype in breast cancers, most notably in BRCA positive (BRCA+) epithelial cells. Oncofetal protein insulin-like growth factor II mRNA binding protein 3 (IMP3) is expressed in many human malignancies. Our lab has recently shown that IMP3 is significantly expressed in triple negative breast carcinomas (TNBC) as compared to other breast carcinomas. The aim of this study is to evaluate the immunohistochemical expression of Slug and IMP3 in BRCA+ breast carcinomas.

Design: Slug and IMP3 immunohistochemistry was performed on BRCA+ invasive breast carcinomas from 1998-2013 on which adequate clinical information was available. Tissue from biopsy and surgical excision specimens was used. Patient age, tumor grade, hormone status and type of BRCA mutation was obtained from our electronic medical record.

Results: 27 tumors were identified and used for the study. 20 were poorly differentiated (Nottingham score 8,9) and 7 were moderately differentiated (Nottingham score 6,7). Age ranged from 24-77 years (mean age $=44) .15 \mathrm{BRCA} 1+, 9 \mathrm{BRCA} 2+, 1 \mathrm{BRCA} 1 \& 2+$ and 2 cases whose BRCA status was not available were identified. Slug was positive in $20(74 \%)$ cases (12 BRCA1+ and 6 BRCA2+) and IMP3 was positive in $15(56 \%)$ cases (12 BRCA1+ and 2 BRCA2+). All 15 cases that were IMP3 positive showed coexpression of Slug and this co-expression was more significant in BRCA1+ carcinomas as compared to BRCA2+ carcinomas ( $\mathrm{P}=.005)$. There were a total of 17 TNBC. Slug and IMP3 were significantly co-expressed in $14(83 \%)$ of TNBC as compared to other carcinomas $(\mathrm{P}<.0001)$. None of the $\mathrm{ER}+$ carcinomas showed reactivity with IMP3. Slug was also expressed in benign epithelial cells (luminal and basal) in terminal duct lobular units of $18(67 \%)$ cases (11 BRCA1+, 6 BRCA2+ and 1 BRCA1\&2+)

Conclusions: A subset of BRCA positive breast carcinomas are known to be triple negative. Our study has demonstrated a previously unreported co-expression of Slug and IMP3 in this subset, most notably in those with BRCA1 mutations. Further larger studies are needed to elucidate the significance of this co-expression which may help to further understand the role of Slug transcription factor in the biology of breast cancer. 
341 Comparison of Dual-ISH (DISH) with Fluorescence In-Situ Hybridization (FISH) and Correlation with Immunohistochemical Findings for HER-2/Neu Status in Breast Carcinoma

ML Wallander, LJ Layfield, SR Tripp. ARUP Laboratories, Salt Lake City, UT; University of Missouri, Columbia, MO.

Background: The predominant methods for determination of HER-2/neu status in breast carcinoma are immunohistochemistry and fluorescence in-situ hybridization (FISH). Both techniques are associated with technical and interpretive difficulties. Alternate methods exist including quantitative PCR and the newly developed dual in-situ hybridization (DISH)

Design: We evaluated HER2 DISH as an alternative to FISH and report our findings from 101 cases. HER2 DISH and FISH were performed according to the manufacturer package inserts. All cases were enumerated by a senior surgical pathologist. Discrepancies between FISH and DISH were resolved by quantitative PCR. Additionally, we correlated HER2 DISH and FISH results with HercepTest and 4B5 immunohistochemistry

Results: Eight cases failed FISH analysis and none failed DISH analysis. A 96\% (89/93) concordance was found between DISH and FISH. In the four discrepant cases, three PCR results agreed with the FISH results and in a single case, PCR agreed with the DISH result. When only HercepTest ${ }^{\mathrm{TM}} 2+$ cases were considered, correlation between FISH and DISH was achieved in 95\% (60/63) of cases. Three of four discrepant cases had a $2+$ HercepTest $^{\mathrm{TM}}$ result.

Conclusions: Based on the $96 \%$ concordance between DISH and FISH it appears that DISH is an acceptable alternative to FISH based in ASCO/CAP guidelines.

342 Basaloid Salivary-Analogue Tumors of the Mammary Gland: Histopathologic Spectrum and Differential Diagnosis

AL Walls, AA Sahin, AK El-Naggar, LP Middleton. MD Anderson Cancer Center, Houston, TX.

Background: Salivary and mammary glands share common ontology, and salivary gland-type tumors of the breast are rare and likely under recognized types of carcinomas. Analogous to primary basaloid salivary tumors, basaloid mammary carcinomas can exhibit a spectrum of changes from benign to malignant. This rarity combined with the spectrum of histologic presentations may not uncommonly lead to diagnostic and management challenges.

Design: Review of 1600 primary breast carcinomas identified ten tumors, seven sent in consultation and three from our institution. Clinical information collected includes age at diagnosis, procedure, treatment, and follow-up. Pathologic parameters included tumor size and axillary lymph node status. Histologic features of each tumor were evaluated by breast and head and neck sub-specialized pathologists independently. Immunohistochemical studies were used to support the histologic diagnosis.

Results: Ten female patients ranged in age from 34-78 (mean 58). Gross size ranged from 0.9 to $6.0 \mathrm{~cm}$ (mean $2.3 \mathrm{~cm}$ ). Four cases were pathologic stage T1 (Stage IA), five T2 (IIA), and one T3 (IIIA). Two showed lymphovascular invasion and two in situ carcinoma. Of seven patients with lymph node evaluation, one had metastases in 7 of 33 nodes. Three tumors each were estrogen and progesterone receptor positive, and none were Her2 positive. Of seven patients with long term follow-up, four had no evidence of disease at 14-121 months (mean 61 months) after diagnosis. One patient is undergoing neoadjuvant chemotherapy 2 months after biopsy. Histologically, all tumors demonstrate basaloid morphology with uniform solid and spindle cell proliferations exhibiting structural and cellular malignant features. Three tumors were classified as low grade salivary-like adenocarcinoma, two as basaloid adenocarcinoma with dedifferentiation, one as basaloid adenocarcinoma ex-monomorphic adenoma, and two as high grade basaloid carcinoma.

Conclusions: Mammary basaloid tumors displayed a spectrum of histologies similar to those of salivary gland origin. These included basaloid salivary-like adenocarcinoma, basaloid adenocarcinoma ex-monomorphic adenoma, dedifferentiated basaloid adenocarcinoma, and basaloid carcinoma, NOS. The latter category may include poorly differentiated carcinoma, solid adenoid cystic carcinoma and undifferentiated neuroendocrine carcinoma. Clinical follow-up revealed a range of outcomes linked to the degree of histologic malignancy. The presentation highlights the salient structural and cellular features that may better help in the diagnosis and classification of these tumors.

343 Ductal Carcinoma In Situ with Distorting Peripheral Sclerosis on Core Biopsy May Be Predictive of Upstage on Excision

LL Walters, JC Pang, L Zhao, JM Jorns. University of Michigan, Ann Arbor, MI.

Background: Several studies have investigated clinicopathologic features in patients with core biopsy (CB) diagnoses of ductal carcinoma in situ (DCIS) in attempt to predict for invasion on subsequent excision as this has significant implications regarding need for axillary staging via sentinel lymph node biopsy (SLNB). This study reexamines this important question.

Design: An electronic database search (1/06-12/11) identified patients with diagnosis of DCIS as the highest stage lesion on CB. Two cases were excluded due to microinvasion upon slide re-review and myoepithelial immunohistochemical staining. Pathologic and clinical features were assessed via slide and chart review, respectively. Distorting periductal sclerosis was defined as irregular angulation of glands involved by DCIS but lacking definite invasion by histology \&/or immunohistochemistry for myoepithelial markers. Preliminary statistical analysis was performed.

Results: Of 186 cases with DCIS as the highest stage lesion on CB $32(17.2 \%)$ had upstage to either microinvasive (9) or invasive (23) ductal carcinoma, with an average size of $0.5 \mathrm{~cm}$ (range $<0.1-1.5$ ). SLNB was performed in 29/32 (90.6\%) of cases with upstage and $55 / 154(35.7 \%)$ without $(\mathrm{p}<.0001)$. Upstage was significantly associated with distorting peripheral sclerosis $(\mathrm{p}=.04)$ and marginally associated with higher linear extent of core involvement by DCIS ( $p=.085$ ).
Figure 1. Examples of DCIS with Distorting Sclerosis (H\&E, 20X)

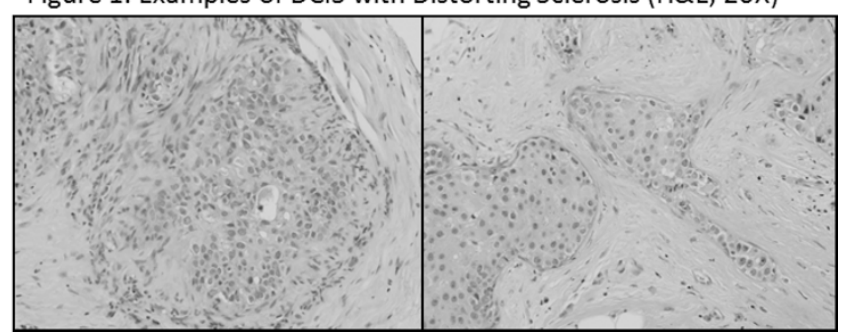

Upstage was not significantly associated with age, laterality, radiologic mass, \# sites biopsied, grade, non-distorting periductal sclerosis or chronic inflammation, cancerization of lobules, or primary DCIS architectural pattern, including extensive comedonecrosis. SLNB was more frequently performed in mastectomy $(56 / 69 ; 81.2 \%)$ vs. breast conserving therapy $(28 / 117 ; 23.9 \%)$ patients $(\mathrm{p}<.0001)$. Reoperation for SLNB was required in $26.2 \%(22 / 84)$ of cases.

Conclusions: SLNB is frequently performed for patients with upstage from DCIS on $\mathrm{CB}$ to microinvasive or invasive carcinoma on subsequent excision. DCIS with distorting peripheral sclerosis without definite invasion on $\mathrm{CB}$ may predict for upstage on excision. This feature may be useful in selecting patients to undergo SLNB at the time of excision to avoid reoperation.

\section{Determination of HER2 Status by Reflex MIP Array}

$Z$ Wang, BP Portier, EC Minca, C Lanigan, GT Budd, RR Tubbs. Cleveland Clinic, Cleveland, $\mathrm{OH}$

Background: Molecular inversion probe (MIP) arrays provide high-quality copy number and genotype data with whole-genome coverage and high resolution of cancer related gene loci. The MIP array assay is specifically designed to work using FFPE tissue due to its utilization of short sequence inversion probes of approximately $40 \mathrm{bp}$ length. In this study, MIP array analysis was performed on a cohort of breast carcinoma which was initially identified as aneusomic due to a CEP17 probe count $>$ 3.0, determined by initial testing of FISH for HER2/CEP17 dual probe (PathVysion, Abbott Molecular, Des Plaines, IL). our data show that utilization of MIP array as a reflex assay for aneusomic FISH cases allows HER2 amplification status resolution and provides additional clinically potentially actionable data on genes with predictive and prognostic significance.

Design: DNA was extracted from FFPE tissue from invasive breast carcinomas following macrodisection. Breast carcinomas utilized in this study segregated into three groups: 1) Aneusomic 2) Monosomic, and 3) Eusomic cases as determined by FISH. Matched patient normal lymph nodes provided reference autologous DNA for a subset of cases from each group. MIP array data was collected using the Affymetrix OncoScan ${ }^{\mathrm{TM}}$ array. Analysis of array data was performed using Nexus software v6 (BioDiscovery Inc., Hawthorne, CA)

Results: MIP array performed on our study cohort revealed great variability in regions of aneusomy along chromosome 17 associated with inconclusive $H E R 2$ status determination by dual probe FISH unambiguously delineated by the MIP array. Reflex testing with MIP array definitely resolved amplification status in all cases $(\mathrm{n}=40)$. In addition to HER 2 status resolution by MIP array, gains and losses in genes involved in modulating therapy were also identified, using this single FFPE based assay.

Conclusions: Use of a single reflex MIP array in breast carcinomas with apparent chromosome 17 aneusomy allowed $H E R 2$ amplification status resolution. MIP array also provided comprehensive genomic coverage for analysis of non EGFR family genes potentially important for prognosis and therapy response prediction. This pilot study shows the feasibility of utilizing MIP array as a reflex assay to resolve $H E R 2$ status in problematic cases identified with chromosome 17 aneusomy (as determined by FISH). This assay allows direct visualization of $H E R 2$ copy number changes and showed high correlation to single probe FISH scoring for $H E R 2$.

345 Number of Pathologically Confirmed Involved Lymph Nodes at Surgery Is a Powerful Predictor of Relapse in Hormone Receptor-Positive, HER2-Nonamplified Breast Cancer Treated by Neoadjuvant Chemotherapy and Followed-Up for 10 Years

$Q$ Wang-Lopez, C Abrial, M-A Mouret-Reynier, P Dubray-Longeras, X Durando, $F$ Kwiatkowski, D Marie-Melanie, A Cayre, N Radosevic-Robin, J-M Nabholtz, P Chollet, F Penault-Llorca. Jean Perrin Cancer Center and ERTICa Research Group, University of Auvergne, Clermont-Ferrand, Auvergne, France; Jean Perrin Cancer Center and INSERM UMR990, University of Auvergne, Clermont-Ferrand, Auvergne, France. Background: In luminal, HER2-nonamplified (HR+/HER2-) breast cancer (BC) treated by neoadjuvant chemotherapy (NACT) it is unclear whether pre- or post-NACT clinicopathologic factors better predict the patient outcome. Moreover, pathologic complete response ( $\mathrm{pCR}$ ), which significantly influences the long-term prognosis, is rarely achieved in the HR+/HER2- BC. In this work we evaluated the importance of pre- and post-NACT parameters for prognosis of HR+/HER2- BC treated by NACT. Design: The study included 128 HR+/HER2- BC patients (pts) treated by taxane or taxane/anthracycline-based NACT in our institution from 2001 to 2007. The following pre-NACT parameters were analyzed: age, clinical tumor size and lymph node (LN) status, histotype, SBR grade and Ki67 index. The post-NACT parameters evaluated were: NACT and surgery type, clinical and pathologic response, presence of tumor cell emboli and number of residually involved LNs at the microscopic review, types 
of adjuvant therapy. Univariate (log rank) and multivariate (Cox regression) statistical analysis was performed. The median follow-up was 101 months (11-152 monts) on August 2013.

Results: In this cohort pCR rate was only $5 \%$ (Chevallier classification, PMID 8338056). Objective (complete plus partial) response rate was $69 \%$, while only $8 \%$ (10 pts) had a complete clinical response after 6 courses of NACT. The number $(\mathrm{N})$ of involved $\mathrm{LN}$ after NACT, separated in groups with $\mathrm{N}=0,1 \leq \mathrm{N} \leq 3$ and $\mathrm{N} \geq 4$, was significant for both disease-free (DFS, $\mathrm{p}=0.0084$ ) and overal survival (OS, $\mathrm{p}=0.021$ ). After an 8 -year follow-up, for the indicated LN number subgroups, the DFS rates were $98.4 \%, 78.8 \%$ and $65.5 \%$, and the OS rates $90.2 \%, 89.5 \%$ and $68.0 \%$, respectively. The recurrence risk (RR) and mortality rate (MR) were increased 3- (CI: 95\% [1.32-6.76]) and 2-fold (CI: 95\% [1.10-3.19]), respectively, in pts with 1-3 involved LN, compared to the pts with no involved LN post-NACT. Similarly, the pts with $\geq 4$ involved LN had the RR and MR 9- and 3.5-fold higher, respectively, than the pts with no involved LN.

Conclusions: The number of involved LN at surgery is a powerful prognostic factor for recurrence and survival in HR+/HER2- NACT-treated BC. Instead of pCR, very rare in this BC subtype, the number of post-NACT metastatic LN can be useful in the choice of subsequent adjuvant treatment.

\section{Elucidating Encounters of Atypical Ductal Hyperplasia Arising in} Gynecomastia

JM Wells, Y Liu, PS Ginter, MP Nguyen, SJ Shin. Weill Cornell Medical College, New York, NY.

Background: Atypical duct hyperplasia (ADH) rarely arises in the setting of gynecomastia. Identification of ADH can be problematic, particularly in active gynecomastia where usual duct hyperplasia (UDH) can be diffuse. We set out to better understand this lesion specifically in this setting from clinical and morphologic standpoints but also evaluate the efficacy of CK5/6 and ER immunostains in distinguishing $\mathrm{ADH}$ from UDH.

Design: 77 cases of ADH arising in gynecomastia were retrieved from our files of which 24 had sufficient material for study. For comparison, 36 cases of gynecomastia with UDH were also studied. Clinical and morphologic review as well as immunostains CK5/6 and ER were performed on each case of ADH and UDH. The extent of CK5/6 luminal epithelial staining was assessed $(0 \%=0,<10 \%=1,10-50 \%=2$ and $>50 \%=3)$. ER was evaluated using the H-scoring system.

Results: All patients were male with an average age of 40 years (range 14-86). Indication for biopsy in ADH patients was breast mass (46\%), "gynecomastia" $(42 \%)$, nipple discharge $(4 \%)$ and calcifications (4\%). In the single case biopsied for mammographically detected calcifications, only ADH was associated with calcifications. Indication for biopsy in patients with UDH was a breast mass $(61 \%)$ and "gynecomastia" (39\%). 50\% of ADH patients with drug histories showed usage known to cause gynecomastia. ADH was most frequently identified in a background of inactive or minimally proliferative gynecomastia (75\%) and involved $20 \%$ of the slide's ducts on average (range 2-70). ADH was more often cribriform pattern (71\%) than micropapillary $(46 \%)$ with a subset demonstrating both $(21 \%)$ whereas all UDH cases showed a micropapillary pattern. Cytomorphologically, cells of ADH were smaller and more monomorphic than those of UDH. The frequency of rare epithelialassociated mitotic figures was similar in $\mathrm{ADH}(21 \%)$ and UDH (31\%). CK5/6 luminal epithelial staining was noticeably decreased (0-1) in ADH (71\%) versus UDH (17\%). Conversely, ADH often showed high ER staining as compared to UDH (H score $>270$ in $88 \%$ and $11 \%$, respectively)

Conclusions: To our knowledge, the clinical, morphologic and immunohistochemical features of $\mathrm{ADH}$ arising in gynecomastia has not been previously reported. We found that in general, $\mathrm{ADH}$ is an incidental finding in a biopsy performed for clinical manifestations of gynecomastia. It is often focal and arises in inactive gynecomastia. If distinction between co-existing UDH is needed, ADH typically exhibits decreased luminal CK5/6 staining and strong/diffuse expression for ER whereas the reverse pattern is seen in UDH.

347 Evaluating the Utility of Trefoil Factor-1 as a Mammary-Specific Immunostain in the Distinction between Carcinoma of Breast and Lung JM Wells, Y Liu, Z Chen, N Narula, SJ Shin. Weill Cornell Medical College, New York, NY.

Background: Discerning metastatic carcinoma of breast origin from primary lung carcinoma (PLC) is important with significant clinical ramifications but can often be difficult due to the lack of reliable breast-specific markers. Mammoglobin and more recently, GATA-3 are two immunostains which have been shown to be most helpful in this regard. Trefoil factor-1 (TFF1) is a protein involved in mucosal defense and repair (gastric) which has also been shown to stain breast carcinoma. We set out to evaluate TFF1's potential as a mammary-specific stain and compare its efficacy with that of mammoglobin and GATA-3 in this diagnostic quandary.

Design: Tissue microarrays of 369 breast carcinomas (BC), all of which were poorly differentiated invasive duct carcinomas and 338 PLC of various histologic types (adenocarcinoma 271, squamous 45 , adenosquamous 8 ) were stained with TFF1 (rabbit, EPR3972, 1:1000, abcam), mammoglobin (mouse, 304-1A5, 1:200, Dako), and GATA-3 (mouse, HG3-31, 1:200, Santa Cruz). Immunohistochemical staining was performed on 4-um thick formalin-fixed, paraffin embedded tissue microarray sections. An H-score (HS) was calculated for each stain in each case. Nonparametric methods were used for statistical analysis.

Results: All three stains were more often positive in BC than PLC with a high degree of statistical significance $(\mathrm{p}<.001)$. This was also true when comparing subtypes of PLC: adenocarcinoma $(\mathrm{p}<.001)$ and squamous $(\mathrm{P}<.001)$. Mammoglobin and GATA-3 were statistically more often positive in BC than in adenosquamous PLC ( $<<.001$ and $\mathrm{p}=.003)$ but not TFF1 $(\mathrm{p}=0.813)$. The area under the receiver-operating characteristic curves for each stain was 0.670 for TFF $1,0.723$ for mammoglobin and 0.886 for GATA-
3. When all possible combinations were analyzed, utilizing all three stains proved to classify with the highest accuracy $(0.913)$.

Conclusions: In daily practice, TFF 1 is not useful as a stand-alone breast-specific marker. However, TFF1 in combination with mammoglobin and GATA-3 can be utilized as part of an ideal immunopanel to distinguish between carcinoma of breast origin from that of primary lung.

348 Analysis of Low-Grade Breast Carcinoma by Oncotype DX: Histology Is Equivalent to Molecular Testing in Predicting Likelihood of Recurrence

SM Wendroth, KA Atkins. University of Virginia, Charlottesville, VA.

Background: Several studies have shown breast cancer recurrence rates can be accurately predicted using molecular markers in patients with estrogen receptor positive, node negative tumors. Oncotype DX (Genomic Health Inc, Redwood City, $\mathrm{CA}$ ) is a commonly used molecular assay designed to evaluate this recurrence risk. The assay evaluates the expression of 21 genes in order to assign a recurrence score (RS) of $0-100$ to the tumor of interest. These scores have been validated to correspond to either low-risk $(<18)$, intermediate-risk (18-30), or high risk $(>30)$ of 10-year distant tumor recurrence. Using this information, patients with high-risk tumors (RS $>30$ ) have been shown to benefit from adjuvant chemotherapy, while those with a lower RS derive no such benefit. The largest group of genes $(5 / 21)$ tested by the Oncotype DX assay corresponds to markers of proliferation. As the Nottingham grading system is a histologic marker of proliferation, this study was designed to determine if Nottingham grades can be utilized to screen breast tumors that are unlikely to have a high-risk RS, and therefore unlikely to benefit from adjuvant chemotherapy. We hypothesized a low Nottingham grade would consistently correlate with an RS of 30 or less.

Design: All low Nottingham grade tumors that had been evaluated with an Oncotype $D X$ assay were retrieved from our institutional archives. While remaining blinded to the recurrence scores all cases were reviewed by a breast pathologist in order to confirm grade. The recurrence scores of the tumors were then unblinded. The percentage of low grade tumors that had an RS $\leq 30$ was calculated with a $95 \%$ confidence interval. Results: Of 19 tumors found to be low-grade by histology, 100\% (95\% C.I. 85.2 - 100\%) had an RS of 30 of less, corresponding to low or intermediate-risk. Seventeen (89.5\%) of the tumors had an RS of less than 18, while only $2(10.5 \%)$ had an RS of $18-30$, corresponding to low- and intermediate-risk of recurrence, respectively.

Conclusions: Due to the high cost of molecular assays the ability to use histologic criteria to screen out patients unlikely to receive additional clinical benefit from these assays is valuable. In our set none of the patients with low-grade tumors had high recurrence scores $(>30)$, and a vast majority $(89.5 \%)$ had low recurrence scores $(<18)$. As adjuvant chemotherapy has only been shown to have definitive benefit in patients with high RS tumors, patients unlikely to have a high RS, and therefore unlikely to benefit from chemotherapy may not need a molecular analysis of their tumor.

349 GATA-3 Expression in Morphologic Subtypes of Breast Carcinoma SM Wendroth, MJ Mentrikoski, MR Wick. University of Virginia, Charlottesville, VA. Background: GATA-3 is a transcription factor involved in the growth and differentiation of a number of human tissues. Clinically, GATA-3 immunohistochemical (IHC) staining has become a useful biomarker for a number of tumors, most prominently bladder and breast primaries. To date no study has looked specifically at the staining pattern of GATA-3 in the different histomorphologic subtypes of breast carcinoma. Thus, we attempted to determine if GATA-3 remained a useful marker of primary breast carcinomas with varying phenotypes.

Design: The archives of our institution were searched for cases of breast carcinoma including the following morphologic subtypes: ductal, lobular, pleomorphic lobular, mucinous, metaplastic, medullary, apocrine, signet-ring, and micropapillary carcinomas. Tissue microarrays (TMAs) were created with four $0.6 \mathrm{~mm}$ punches per case. These TMAs were cut at 5 microns and stained with monoclonal GATA-3 antibody (Biocare Medical Inc, Concord, CA). The tumors were considered positive if greater than $5 \%$ of the cells showed nuclear staining, and scored semiquantitatively.

Results: Of the 55 ductal carcinomas, 51 (92.7\%) expressed GATA-3. Of note, all four negative tumors were Nottingham grade III. GATA-3 was expressed in 28 of 29 (96.6\%) lobular carcinomas, 10 of $11(90.9 \%)$ apocrine carcinomas, 10 of $12(83.3 \%)$ medullary carcinomas, 5 of $9(55.5 \%)$ metaplastic carcinomas, and 1 of 2 signet ring cell carcinomas. All mucinous carcinomas (23/23) and micropapillary (12/12) variants were positive with strong staining intensity.

Conclusions: While the vast majority of ductal carcinomas expressed GATA-3, all negative tumors were of Nottingham grade III. Additionally, the higher grade tumors had weaker staining intensity compared to the lower-grade ductal carcinomas. We also found strong positivity in all mucinous carcinomas, another low-grade carcinoma with a good prognosis. Despite this, all micropapillary carcinomas, high-grade tumors with a poor prognosis, also stained positive. Medullary and metaplastic carcinomas were the least likely to show GATA-3 expression at $83.3 \%$ and $55.5 \%$, respectively. For this reason, these morphologic subtypes cannot be confidently ruled out on a negative GATA-3 stain.

350 GATA3 Expression Profile in Invasive Breast Carcinoma Post NeoAdjuvant Systemic Chemotherapy

PA Williams, S Islam. University of Ottawa and Eastern Ontario Regional Laboratory Association (EORLA), Ottawa, ON, Canada.

Background: Immunohistochemistry for GATA3, a transcription factor, has become a new favourite for the diagnosis of metastatic breast carcinoma. Large studies have shown that between 84.5 and $94 \%$ of breast carcinomas are positive for GATA3. In addition to being expressed in breast carcinoma, GATA3 is also expressed in urothelial carcinoma, and recently reported in up to $51 \%$ of salivary gland neoplasms (including $100 \%$ of 
all salivary duct carcinomas). It has been shown to have negligible expression in other tumours. GATA3 has been previously demonstrated to be positive in all metastatic breast carcinomas that have GATA3 positive primaries. GATA3 expression is positively linked to estrogen receptor (ER) expression. ER positive breast carcinoma is well documented to potentially lose ER expression post neo-adjuvant systemic chemotherapy, and ER status is retested in the post-treatment resection specimen to guide hormonal therapy. To date, there have been no studies examining how systemic chemotherapy affects expression of GATA3.

Design: All cases of post neo-adjuvant breast carcinoma surgically resected at The Ottawa Hospital between June 2010 and September 2013 were reviewed. A total of 100 cases were identified that had paraffin embedded tissue blocks available for both pre-treatment biopsies and post-treatment resections. All cases had ER status recorded previously. Each specimen was reacted with antibodies for GATA3 and reactivity was recorded for pre-treatment and post-treatment specimens. Expression profiles were created for each paired biopsy and resection for GATA3 and ER. GATA3 expression was rated as $3+$ for strong diffuse nuclear staining, $2+$ for moderate diffuse nuclear staining and $1+$ for focal/weak nuclear staining.

Results: Preliminary results of 20 cases demonstrated that in the pre-treatment biopsies, all had nuclear reactivity for GATA3. When compared to the post-systemic chemotherapy tumours, GATA3 expression was also retained in all cases. GATA3 expression was however, decreased $(2+$ and $1+)$ in cases that were ER negative posttreatment.

Conclusions: We conclude that GATA3 expression is maintained in breast carcinomas post neo-adjuvant systemic chemotherapy that were GATA3 positive on biopsy. Loss of ER positivity post systemic chemotherapy decreases GATA3 staining, but does not result in a complete loss of reactivity. Immunohistochemistry for GATA3 is therefore useful in identifying potential breast carcinoma metastases post systemic chemotherapy, even with loss of ER reactivity.

\section{Nuclear H\&E Staining Pattern in Flat Epithelial Atypia of the Breast Predicts Presence of Carcinoma on Excision: A Digital Image Based Histopathologic Analysis}

PA Williams, B Djordjevic, Y Ayroud, S Islam, D Gravel, CE Parra-Herran. University of Ottawa, The Ottawa Hospital, Ottawa, ON, Canada.

Background: Diagnosis of flat epithelial atypia (FEA) on biopsy has prognostic and therapeutic relevance, given its documented association with breast cancer. However, rates of malignancy in subsequent excision are low in most studies, and to date, there are no morphologic or immunohistochemical features that can separate cases of FEA with concomitant breast carcinoma from those otherwise benign. We present a histopathologic study aiming to identify morphologic features unique to cases of FEA associated with cancer using digital image analysis.

Design: Resection specimens (lumpectomy, mastectomy) containing FEA were retrieved from our files. Diagnosis was confirmed by two breast pathologists and one senior resident using strict established morphologic criteria. Cases were divided in two groups: FEA associated with situ and/or invasive ductal and/or lobular carcinoma (FEA-C, $\mathrm{n}=30$ ) and FEA without malignancy (FEA-0, n=28). Slides were digitally scanned (Aperio), TIFF images from two representative areas at 20x magnification were obtained and analyzed using digital software (Zen 2011, Carl Zeiss Microscopy, Germany). Several nuclear features were measured, including diameter, area, perimeter, convexity, ellipse angle, feret ratio, roundness, grey, blue, red and green levels. Collected data was analyzed using statistical software (SPSS v 17).

Results: Parameters related to nuclear shape and size (area, diameter) were similar in both groups. However, FEA-C cases had signigicantly higher densitometric green $(\mathrm{p}=.01)$, red $(\mathrm{p}=.04)$, blue $(\mathrm{p}=.03)$ and grey $(\mathrm{p}=.02)$ scale levels compared to FEA-0 cases, indicating that nuclei in FEA-C are less dense (more open) than nuclei in FEA0.13 randomly selected images from each group were examined by two additional breast pathologists, who blindly classified them based on nuclear staining alone as $\mathrm{H}$ (Heterogeneous/open chromatin pattern with inconspicous nucleoli) or U (Uniform chromatin pattern without visible nucleoli). Neither pathologist labelled the 13 FEA0 images as $\mathrm{H}$, whereas 10/13 FEA-C images were labelled as $\mathrm{H}$ by one or both pathologists $(100 \%$ specificity and positive predictive value of " $\mathrm{H}$ " staining pattern in diagnosis of FEA-C).

Conclusions: Based on digital image analysis and pathologist evaluation using routine microscopy, we conclude that nuclear staining pattern is the most important morphologic parameter of FEA in predicting concomitant carcinoma. Thus, FEA nuclear H\&E staining pattern may potentially serve as a guide for clinical management (observation versus excision).

352 Response to Neoadjuvant Chemotherapy: Composite Computational and Histochemical Analyses Define Important Determinants of Pathological Response

A Witkiewicz, D Euhus, V Sarode, J Franco, E Knudsen. UT Southwestern, Dallas, TX Background: Neoadjuvant chemotherapy is widely used in the treatment of breast cancer. In this context, a pathological complete response (pCR) is known to portend a good prognosis. However, the determinants of this response remain elusive. Therefore, markers of response to chemotherapy are particularly important to define patients who will benefit vs. those who by other interventions.

Design: A collection of $>1,000$ cases treated with neoadjuvant chemotherapy were evaluated using computational approaches. Individual genes were associated with pCR by an iterative AUC analysis to rank genes by there impact on response to therapy. Gene ontology and pathway analysis defined processes associated with pCR and residual disease. Preclinical analyses were used to evaluate key pathways defined from the computational analysis. Lastly, top hits associated with either pCR or residual disease were validated by immunohistochemical analysis in independent patient cohorts.
Results: Multiple genes that were strongly associated with pCR or residual disease were identified. In particular a host of gene involved in cell cycle and regulated by $\mathrm{RB} / \mathrm{E} 2 \mathrm{~F}$ were associated with pCR. Preclinical models demonstrated that loss of RB was associated with increased sensitivity to chemotherapy, while pharmaceutical RB activation protects from chemotherapy mediated cytotoxicity. Computational approaches revealed the $\mathrm{RB} / \mathrm{E} 2 \mathrm{~F}$ target gene $\mathrm{DEK}$ as a top determinant of $\mathrm{pCR}(\mathrm{p}<0.001)$. This observation was validated in independent cohorts, wherein high DEK expression was a potent determinant of pCR. Interestingly, residual disease was associated with a diverse series of genes with no clear ontology. BCAM was one of the top ranked genes associated with residual disease, and we found that BCAM added additional specificity to markers associated with pCR. Together multi-marker approaches yielded the best utility in predicting response to neoadjuvant therapy.

Conclusions: These data demonstrate that the RB/E2F pathway is a key determinant of response to neoadjuvant chemotherapy, and that DEK is a particularly potent marker of response. Genes associated with resistance to chemotherapy can be used to further enhance the sensitivity and specificity of such markers. Prospective clinical studies ware ongoing to evaluate the composite efficacy for this work.

353 Expression of HER2/Neu in Ductal Carcinoma In Situ and Its Correlation with Clinical Pathological Factors

$J X u, K M$ Parker. Baptist Health System, Birmingham, AL.

Background: Globally, breast carcinoma is the most common malignancy and the leading cause of cancer death in women. HER2/neu status has been regarded as an important independent predictive marker in breast carcinoma and a clinically useful parameter in determining treatment options. Despite its prognostic value in invasive cancer, the clinical implication of HER2/neu expression in ductal carcinoma in situ (DCIS) has yet to be clearly elucidated.

Design: We aim to analyze the relationship between HER2/neu expression status in DCIS and some clinical pathological parameters including HER2/neu status in invasive component, histological grade, tumor size and axillary lymph node involvement.

Results: Eighty three modified radical mastectomy specimens having both DCIS and invasive ductal carcinoma (IDC) components were included in this study. The mean age of the patients was 61.7 years. In 44 cases without HER2/neu overexpression in DCIS foci, only one showed its overexpression in IDC foci; whereas in 39 cases showing HER2/neu overexpression in DCIS, 36 had overexpression in their associated IDC components $(\mathrm{p}<0.0001)$. High histological grade DCIS had more HER $2 /$ neu overexpression than low grade DCIS $(55.8 \%$ vs. $32.3 \%, p<0.05)$. In the cases with IDC size smaller than $2 \mathrm{~cm}, 35 \%$ had HER $2 /$ neu overexpression in DCIS foci; whereas in the cases with IDC size larger than $2 \mathrm{~cm}, 58 \%$ had HER2/neu overexpression in DCIS foci $(\mathrm{p}<0.05)$. The axillary lymph node positive cases had higher DCIS HER2/ neu overexpression percentage than axillary lymph node negative cases $(64.1 \%$ vs. $39.3 \%, \mathrm{p}<0.05)$.

Conclusions: HER2/neu expression status in DCIS is highly correlated with its expression in IDC developed in the same patient. In addition, HER 2 /neu overexpression in DCIS is found to be significantly associated with DCIS histological grade, IDC tumor size, axillary lymph node involvement. Assessment of HER2/neu expression in DCIS might be considered a useful predicting parameter in breast cancer.

354 Management of Breast Papillary Lesions Diagnosed at UltrasoundGuided Vacuum-Assisted and Core Needle Biopsies

$R$ Yamaguchi, M Tanaka, GM Tse, $H$ Yano. Kurume University School of Medicine, Kurume, Japan; Social Insurance Kurume Daiichi Hospital, Kurume, Japan; The Chinese University of Hong Kong, Hong Kong, Hong Kong.

Background: To assess the diagnostic accuracy and outcome of breast papillary lesions diagnosed by ultrasound-guided core needle biopsy (NB) or vacuum-assisted large volume "mammotome (MT)" biopsy, and whether no surgical excision is justified in non-malignant papillary lesions so diagnosed.

Design: Among totally 3219 (MT2195/NB1024) breast biopsies spanning 5 years (2007-2011), all papillary lesions were selected and reviewed for (i) their initial diagnoses as benign, atypical, or malignant, and (ii) the subsequent treatment received (some were excised, the others were followed-up until 2012 with at least one interval imaging examination).

Results: One hundred eighty-five (5.7\%) papillary lesions [MT164(89\%)/NB21(11\%)] were identified.

(i) One hundred forty-two cases (77\%. MT/NB:125/17, respectively) were benign, 24 $(13 \% .23 / 1)$ were atypical and $19(10 \% .15 / 4)$ were malignant.

(ii) Of the 142 benign cases, 106 had imaging follow-up (FU) and 17/106 cases were excised. Three were excised immediately post-biopsy, all were truly benign. The other 14 were excised after interval imagings showed size/shape changes, 9 were truly benign [FU period 2-38 months] and five were malignant [FU period 4-57 months], including three with only minute papillary fragments (two papillary DCIS with microinvasion and one invasive cancer at final excision) and two papillomas with DCIS. Of the 89 benign cases with only imaging FU, there was no upgrade in diagnosis with interval imaging [FU period 5-71 months]. The difference of the upgrade rate between MT (5/95) and NB (0/11) was not significant. Of the 24 atypical cases, 23 had FU. Of these, 19 were excised; excision diagnoses were benign in $6(32 \%)$ and malignant in $13(68 \%)$ cases ( 9 papillary DCIS and 4 invasive carcinoma). The remaining 4 cases that were not excised did not show changes at FU imaging, and were considered non-malignant. The difference of the upgrade rate between MT (12/22) and NB (1/1) was not significant. Of the 19 malignant cases, 18 were excised (one lost to FU); excision diagnoses were all malignant (14 papillary DCIS and 4 invasive carcinomas). 
Conclusions: MT was not superior to NB in diagnostic accuracy in benign and atypical papillary lesions. Benign papillary lesions diagnosed by MT or NB might not require immediate excision, but should receive imaging FU. Excisions should be performed in cases showing changes in imaging features, as there was a $5 \%$ upgrade rate at excision.

355 Mitochoncdrial DNA Damage Triggers Tumor Progression and Metastasis in PyMT Mice

L Yuzefovych, AG Kahn, GL Wilson, L Rachek. College of Medicine, University of South Alabama, Mobile, AL; University of South Alabama, Mobile, AL.

Background: Breast cancer is the leading cause of cancer death among women worldwide. On a molecular level, it is unclear which of the changes in breast tumors are likely to lead to invasion and metastasis. Among the potential targets are mitochondria, generation of mitochondrial reactive oxigen species (ROS) and mitochondrial DNA (mtDNA). The objective of this study is to determine the involvement of mtDNA damage in breast cancer progression and if the targeting of the DNA repair enzyme hOGG1 into mitochondria prevents tumor progression in a genetic model of breast cancer.

Design: Four groups of female double transgenic mice were generated in our laboratory. We have used the F1 cross of a well-characeterized preclinical transgenic model of metastatic breast cancer male mice (PyMT) with females: 1) Tg, overexpressing a human mitochondrial repair enzyme (hOGG1) in addtion to their endogenous one (OGG1) $(\mathrm{n}=13) ; 2) \mathrm{KO} / \mathrm{Tg}$, knockout mice lacking endogenous OGG1 and overexpressing hOGG1 ( $\mathrm{n}=10)$; 3) KO, knockout mice lacking endogenous OGG1 (n=21); 4) WT, wild type with endogenous OGG1 $(\mathrm{n}=14)$. Starting at 16 weeks mice were sacrified and all mammary tumors and lungs removed. The tissue was fixed in formalin, paraffin embedded, and stained with hematoxylin-eosin. The breast neoplasms were classified according to the Annapolis consensus. The number of lung metastastatic foci was recorded. Statistical analysis was performed using ANOVA.

Results: The results are summarized in Table 1.

Table 1

\begin{tabular}{|c|c|c|c|}
\hline PyMT Mice Genotype & $\begin{array}{l}\text { Primary Tumor } \\
\text { Incidence }\end{array}$ & Metastatic Incidence & $\begin{array}{l}\text { Average Number of } \\
\text { Metastatic Lung Foci }\end{array}$ \\
\hline $\mathrm{KO}$ & $100 \%$ & $82 \% *$ & $13.29 \pm 2.75$ \\
\hline WT & $100 \%$ & $62.5 \%$ & $11.27 \pm 2.04$ \\
\hline $\mathrm{Tg}$ & $100 \%$ & $33.3 \% *$ & $0.66 \pm 0.15 *$ \\
\hline $\mathrm{KO} / \mathrm{Tg}$ & $71 \% *$ & $36.4 \% *$ & $1.27 \pm 0.3^{*}$ \\
\hline
\end{tabular}

$* \mathrm{p} \leq 0.05$ vs WT group, one way ANOVA

All the neoplasms were advanced invasive carcinomas. A two fold difference in the metastatic incidence in the lungs was noted between the PyMT/Tg and PyMT/WT mice. Lungs from the PyMT/Tg mice had about a 15 fold decrease in the average number of metastatic foci compared to PyMT/WT mice. These differences were statistically significant $(\mathrm{p} \leq 0.05)$

Conclusions: Based on these data we conclude that mtDNA damage appears to play a role in both progression and the proliferation of distant metastatic foci. These results have potential clinical and translational significance as most current therapies increase oxidative stress, therefore inducing more damage to mtDNA, increase ROS and thus could drive tumor metastasis.

\section{Cardiovascular Pathology}

356 Gross Examination of the Left Ventricular Assist Device: A Primer for the Pathology Resident

V Ananthanarayanan, MK Mirza, MB Alikhan, AS Akki, AN Husain. University of Chicago, Chicago, IL.

Background: The advent of left ventricular assist device (LVAD) placement has significantly reduced mortality in patients with cardiac disease. As this translates into an increased incidence of LVAD associated autopsies and explanted hearts, the pathology resident needs an insight into the structural and mechanical aspects of this device as well as its contribution to morbidity and mortality of the patient. Current literature does not elucidate algorithms towards LVAD examination at autopsies. We address the dearth of this information by providing a summary of our experience with LVAD-associated autopsies and present a guide to the examination of these specimens.

Design: Nineteen consecutive LVAD-associated autopsies were included in this study. We tabulated clinical information and carefully examined segments of the device, noted intactness of anastomoses, presence of pre-mortem thrombi, significant hemorrhage and evidence of infection. The heart was dissected as shown in figure 1 . Step 1: gross examination of LVAD-aortic anastomosis and LVAD-apex anastomosis. 2. Inspection for thrombi in ventriculo-aortic connection. 3. Analysis of coronary arteries. 4. Dissection along ventral axis to apex. 5 Breadloafing of tissue from apex to base. 6. Removal of LVAD parts for internal inspection. 7. Sampling for histologic examination as appropriate.

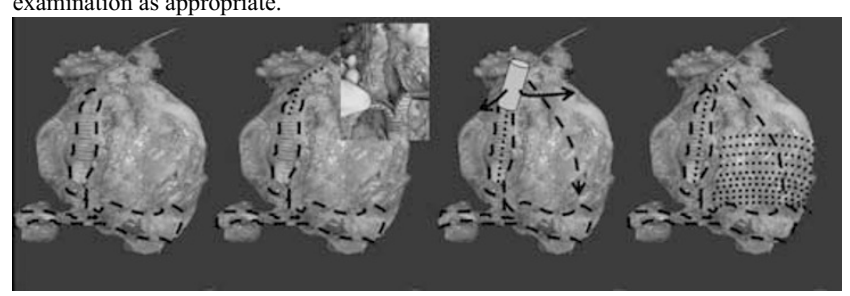

Results: In all 19 cases $(100 \%)$ suture lines were intact and no defective LVAD was identified. Clinically significant LVAD thrombi were noted in 2/19 cases
$(10 \%)$, significant hemothorax in $1 / 9(5 \%)$ and 1 case revealed encroachment of the endomyocardium over the LVAD inflow at cardiac apex, leading to functional obstruction (795 days post LVAD).

Conclusions: Correct examination of the LVAD associated cardiac specimen is a crucial component of the medical autopsy. Our dissection approach provides a useful tool in effectively analyzing all potential parts of the device as well as evaluation of the native heart by gross and histologic techniques. In our experience, there was no case of anastomotic leak or death due to a functionally defective LVAD. Although LVAD placement is generally considered a 'bridge' to cardiac transplant, long standing devices may be susceptible to endomyocardial encroachment and functional obstruction.

357 Lipomatous Lesions of the Heart: A Comparative Morphologic and Cytogenetic Study with New Pathogenetic Insights

MC Bois, AM Oliveira, CW Roth, BR Evers, RN Wehrs, JJ Maleszewski. Mayo Clinic, Rochester, MN.

Background: Benign lipomatous lesions of the heart encompass an etiologically diverse group of entities, including neoplastic, congenital, and reparative phenomena. Among these, lipomas and lipomatous hypertrophy of the atrial septum (LHAS) represent two commonly encountered, both presenting as masses. While cardiac lipomas are believed to be neoplastic, there is little supporting genetic evidence. LHAS is thought to represent embryologically entrapped fat that increases in size proportional to visceral adiposity, though supporting genetic data is absent here as well. To date, no study has systematically and comparatively evaluated the morphologic and genetic characteristics of these lesions.

Design: Tissue registry archives of Mayo Clinic were queried for cases of cardiac lipoma and LHAS (1994-2011). Three cases of ventricular fatty infiltration were identified from age-matched autopsy subjects for controls. Clinical, imaging and pathological findings were reviewed. Representative cases in each cohort were evaluated by fluorescence in situ hybridization (FISH) for $H M G A 1$ and $H M G A 2$ loci rearrangement via break-apart probes and for $C P M / M D M 2$ locus amplification via enumeration probes.

Results: Four cases of cardiac lipoma were identified (mean age 59 yrs, range 48-64; 2 men); 2 right atrial, 1 left ventricular and 1 pericardial. 42 cases of LHAS were identified (mean age $75.6 \mathrm{yrs}$, range 45-95, 20 men), 39 of which were autopsy-derived. The majority of these lesions were identified incidentally on imaging or at the time of surgery/autopsy (lipomas, 3; LHAS, 40). The median size was $6.1 \mathrm{~cm}$ for lipomas, and $2.8 \mathrm{~cm}$ for LHAS $(\mathrm{n}=14)$. A single case each of a cardiac lipoma and LHAS were found to harbor $H M G A 2$ rearrangement, while no case showed molecular cytogenetic abnormality of $H M G A I$ or $C P M$. No cases of fatty infiltration exhibited the evaluated cytogenetic rearrangements.

Conclusions: This represents the largest series of histopathologically confirmed cardiac lipomas from a single institution. Additionally, it is the first to evaluate cardiac lipomas for genetic alterations associated with extracardiac lipomoutous lesions. The genetic and morphologic similarities found provide evidence in support of both the neoplastic nature of cardiac lipomas as well as highlighting etiologic similarities in these tumors. This is also the first study to evaluate LHAS and fatty infiltration for cytogenetic alterations. Interestingly and unexpectedly, a single case of LHAS contained an HMGA2 rearrangement, challenging the currently accepted hypothesis of pathogenesis for this lesion.

358 An Evaluation of the Utility of Post-Mortem Computed Tomography in the Diagnosis of Lethal Coronary Artery Atherosclerosis and Hypertensive Heart Disease

CR Curry, VS Snyder, SW Andrews, SL Lathrop, EW Matshes. University of Chicago and NorthShore University HealthSystem, Evanston, IL; University of New Mexico and the Office of the Medical Investigator, Albuquerque, NM.

Background: Autopsy is regarded as the gold standard for the diagnosis of natural disease and trauma in the postmortem setting. Despite this, some centers routinely use postmortem computed tomography (PMCT) scans. Amongst the most common causes of death in any medical examiner jurisdiction are atherosclerotic cardiovascular disease (ASCVD) and/or hypertensive cardiovascular disease (HSCVD). Data does not exist to support or refute the premise that routine use of PMCT is useful for the detection of hypertensive and atherosclerotic cardiovascular disease (HASCVD). Practical experience shows that non-contrast PMCT does not accurately document or diagnose lethal coronary artery atherosclerosis (CAA) and sequelae, nor does it allow for the diagnosis of hypertensive cardiovascular disease.

Design: One hundred adult forensic autopsies were selected from an 18-month period (July 2011 to January 2013). The cases were divided into two age- and sex-matched groups by cause of death: (1) those due to HASCVD, and (2) those due to other causes. Two forensic pathologists, blinded to the cause of death, reviewed pre-autopsy PMCT scans of the chest and recorded the presence or absence of severe coronary artery stenosis (defined as $>75 \%$ stenosis), myocardial pathology (including left ventricular hypertrophy and myocardial infarction), cardiomegaly, and coronary artery calcium deposition. The same set of data was obtained from the corresponding autopsy reports. Results of the PMCT interpretations were compared with the results obtained from autopsy.

Results: PMCT did not detect clinically significant CAA and completely missed it in 56 cases where it was proven at autopsy. PMCT did not detect myocardial pathology in 50 cases where it was proven at autopsy and mistakenly detected it in 5 cases where it was not identified at autopsy. PMCT did not detect cardiomegaly in 44 cases where it was identified at autopsy and mistakenly detected it in 5 cases where it was not identified at autopsy. Coronary artery calcium deposition was not identified at autopsy in 46 cases where it was detected by PMCT. 